$$
\begin{aligned}
& \text { U. SRUDEOIL NATURAL GAS, } \\
& \text { * AND NATURAL GASLIQUIDS } \\
& \text { RESERVES }
\end{aligned}
$$

1997 ANNUAL REPORT
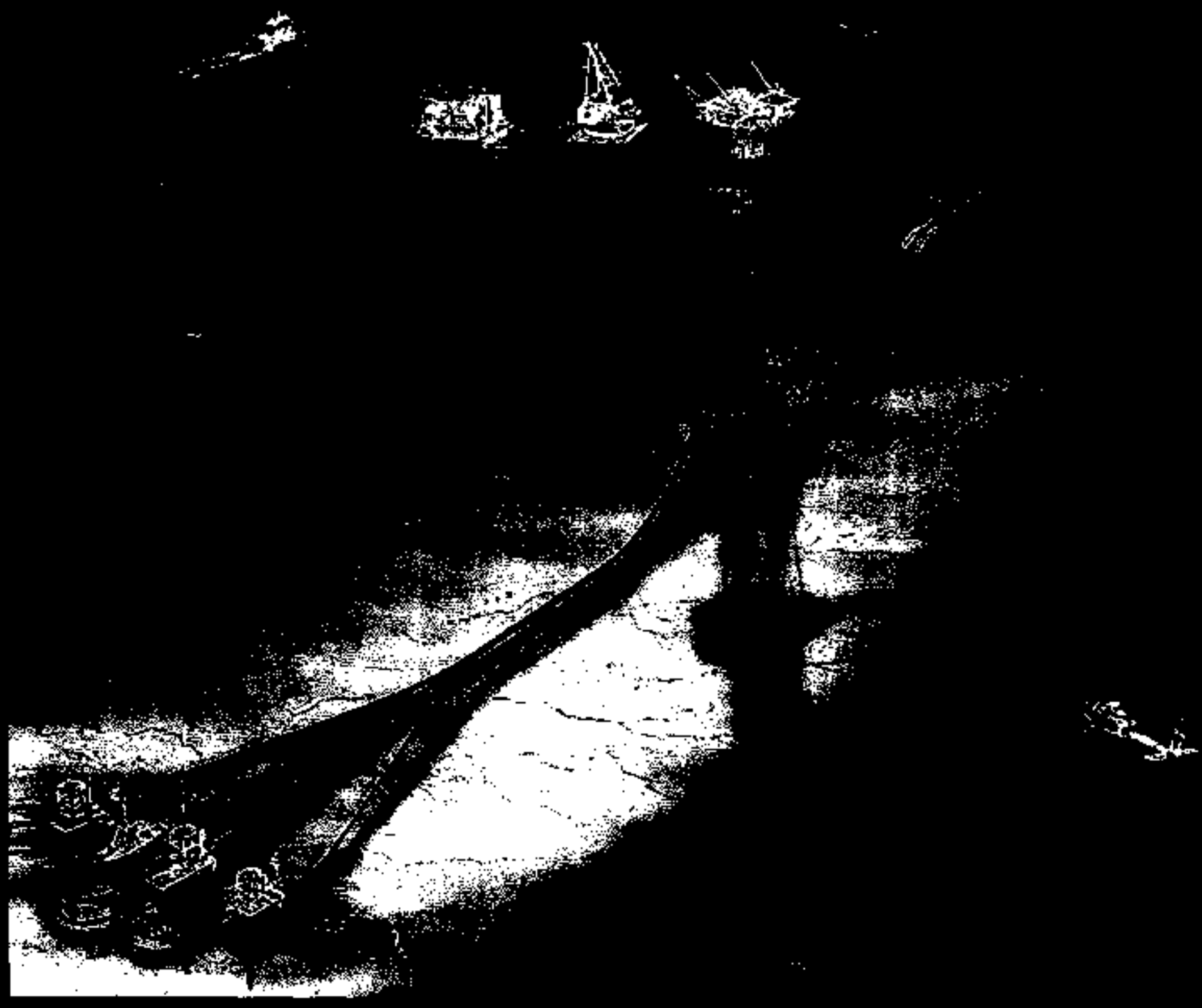

-

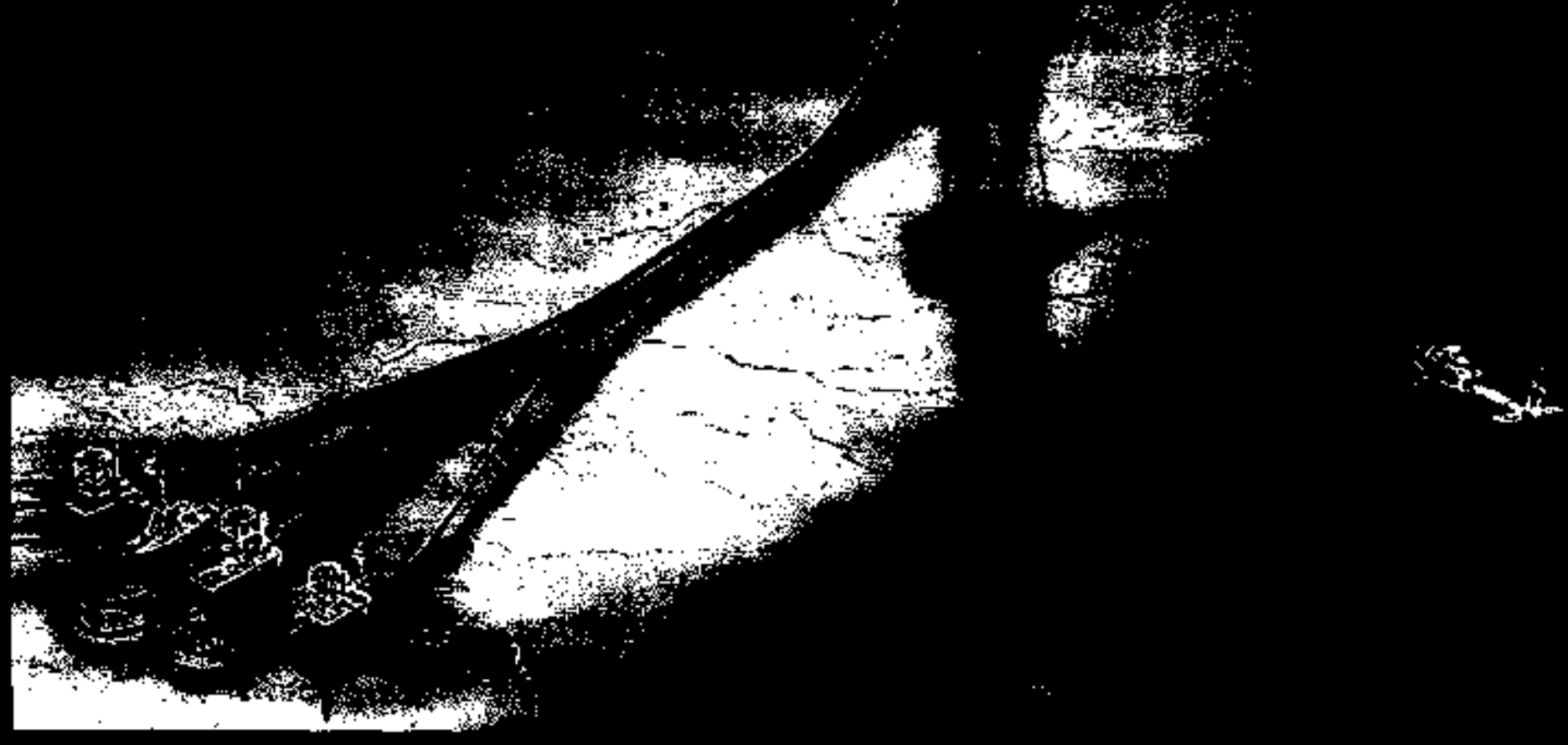

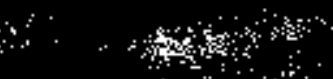




\section{HOW TO OBTAIN EIA PRODUCTS AND SERVICES}

For further information on any of the following 5ervizes, or for answ'ers to etergy information questions, please contaci E]A's Nattonal Energy Information Center

Nattonal Encrgy Information Center (NEIC)

(202) $586-8800$

Energy lnformation Admınıstratton

(202) 586-0727 (fax)

EJ-30. Fortestal Bulding

T"TY (202) 586-1181

Washington, DC 20585

E-mal 1afoctr@era doe gov

\section{Electronc Products and Services}

ELA's Internet Site Services offer nearly all ElA puthications Users can view and downioad selected pages or enttre reports, search for unformation, download F./A data and analysis applicatıons and find out about new FiA information products and services

World Wide Web http://w a w.ela.doe.g jv

FTP: ftp $/ /$ ftp.eza.doe.gov

ElA also offers a listserve sesvice for ElA press releases and other short documents Sign up on the EIA World Wide Web site

EIA's CD-ROM, Energy InfoDise contaus thost ElA publications and major energy database applications The Energy InfoDtsc, produced quarterlv is aqadalis for a fee from STAT-USA, Department of Commerce, 1-800-STAT-USA

The Comprehensive Oul and Gas Information Source (COGIS), a bulletun board service, contairs data files from most of ELA's oul-and gas-related reports It ts avall sble for a fee from STAT-USA, on I-800-STAT-USA

\section{Prunted Publicatıons}

EIA directories are avalable free of cllarge from NEIC Recent periotucals and one-tıme reports are avalable from the Government Printing Office Older reports are avalable from the National Technical Information Service

Superıntendent of Documeits

1) S Government Psuntung Office

PO Box 371954

Pittsburgl, PA 15250.7554

(202) $512-1800,(202)-512-2250(\mathrm{fax})$
National Techuca] Iaformaton Service

U S Departisent of Commerce

Springfteld, VA 22161

$\$ 285$ Port Royal Road

I-(800) 553-6847, (703) $321-8547$ (fax)

\section{Cover Phato Credit:}

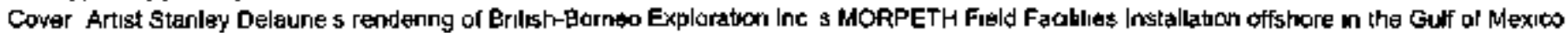

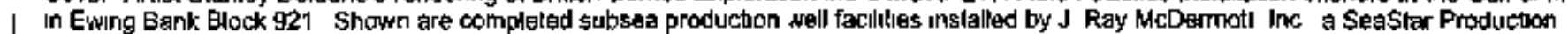

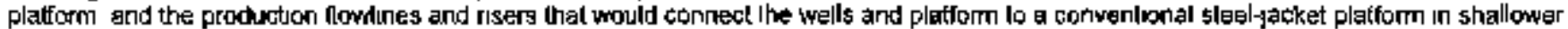

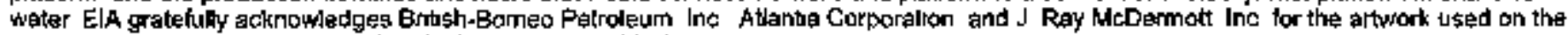
cover of this report (Antwark (0) Ray tictDermolt Inc 1997) 


\title{
U.S. Crude Oil, Natural Gas, and Natural Gas Liquids Reserves
}

\section{Annual Report}

\author{
December 1998
}

Energy Information Administration

Office of Oil and Gas

U.S. Department of Energy

Washington, DC 20585

MASTER

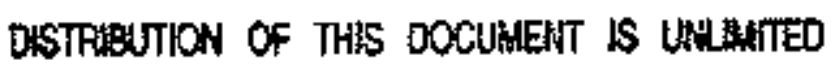

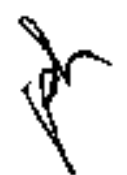

Thls report wes prepared by the Energy Information Administratton, the independent steilsticad and andilyteal agency within the U.S. Department of Energy. The information conkained hereln should be allribuled to the Energy Intormation Adminstration and should not be construed as advocaling or reflecting any policy position of the Department of Energy or any other orgarization. 



\section{DISCLAIER}

This toport wes propared at an acoount of work sponsoced by an spency of the

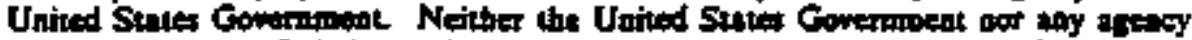

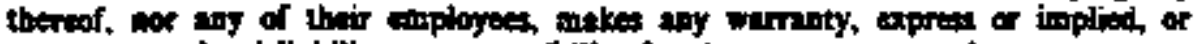

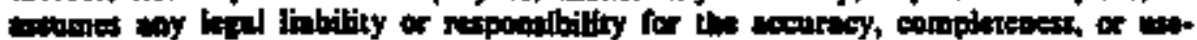

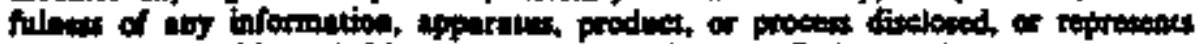

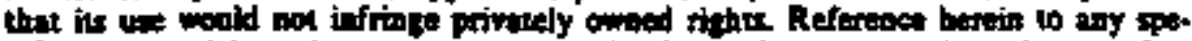

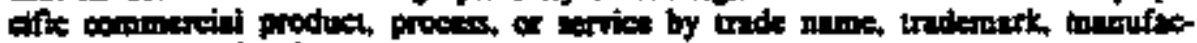

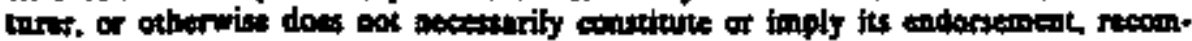

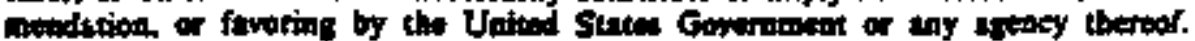

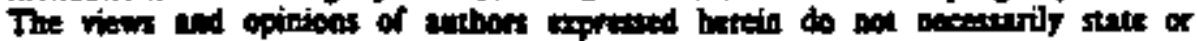

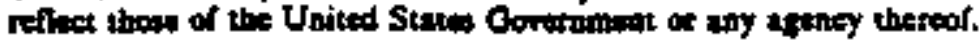




\section{DISCLAIMER}

Portions of this document may be illegible in electronic image products. Images are produced from the best avallable original document. 


\section{Preface}

The U.S. Crude Oil, Natural Gas, and Natural Gas Liquids Reserves 1997 Annual Report is the 21st prepared by the Energy Information Administration (EIA) to fulfill jts responsibility to gather and report annual proved reserves estimates. The EIA annual reserves report series is the only source of comprehensive domestic proved reserves estimates. This publication is used by the Congress, Federal and State agenctes, industry, and other irterested parties to obtain accurate estimates of the Nation's proved reserves of crude oil, natural gas, and natural gas liquids. These data are essential to the development, implementation, and evaluation of energy policy and legislation.

This report presents estimates of proved reserves of crude oil, natural gas, and natural gas liquids as of December 31, 1997, as well as production volumes for the United States and selected States and State subdivisions for the year 1997. Estimates are presented for the following four categories of natural gas: total gas (wet after lease separation), nonassociated gas and associated-dissolved gas (which are the two major types of wet natural gas), and total dry gas (wet gas adjusted for the removal of liquids at natural gas processing plants). In addition, reserve estimates for two types of natural gas liquids, lease condensate and natural gas plant liquids, are presented. The estimates are based upon data obtained from two annual ElA surveys: Form EIA-23, "Annual Survey of Domestic Oil and Gas Reserves" and Form ELA-64A, "Annual Report of the Origin of Natural Gas Liquids Production." Atso included is information on indicated additional crude oil reserves and crude oil, natural gas, and lease condensate reserves in nomproducing reservoirs. A discussion of notable oil and gas explotation and development activities during 1997 is provided.

The appendices contain data by operator production size class for crude oil and natural gas reserves and production; the top 100 U.S. fields ranked within an oil or gas proved reserves group for 1997; report Table i converted to metric units; historical State data; a summary of survey operations; a discussion of statistical considerations; methods used to develop the estimates provided in this report; maps of selected State subdivisions; and examples of the survey forms. A glossary of the terms used in this report and in survey Forms ElA-23 and ELA-64A is provided to assist readers in more fully understanding the data.

This annual reserves report was prepared by the Reserves and Production Division (located in Dallas, Texas), Office of Oil and Gas, Energy Information Administration. General information regarding preparation of the report may be obtained from Kenneth A. Vagts, Director, Office of Oil and Gas and Johr H. Wood, Director, Reserves and Production Division (214-720-6160).

Authors were foln $\mathrm{H}$. Wood, Steven G. Grape and Rhonda S. Green. Technical contributions were made by Rafi $M$. Zeinalpour and technical editing was provided by David F. Morehouse.

Address questions on specific sections of the publication to the following analyst/author:

- Executive Summary and Appendices F and G John H. Wood

Phone: $214 \cdot 720 \cdot 6160$

E-mail: jwood@eiadoe.gov

Fax: 214.720.6155

- Chapter 2, 3, 4,5

Steven G. Grape

Phone: $214 \cdot 720 \times 6174$

E-mail: sgrapeseia.doe-gov

Fax: 214-720-6155

- Chapter 1, Appendices A, B, C, D, E, H, and 1

Rhonda 5. Green

Phone: 214.720-6161

E-maili rgreengeja.doe.gov

Fax 214.720-6155

- Field-level Reserves Quality Assurance

Rafi M. Zeinalpour

Phone: 214.720.6191

E-mail: rzeinalpoeja.doe.gov

Fax 214.720.6155 



\section{Contents}

Page

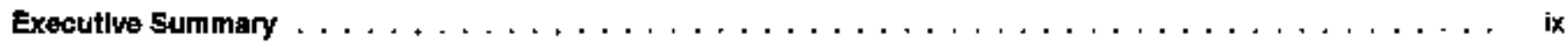

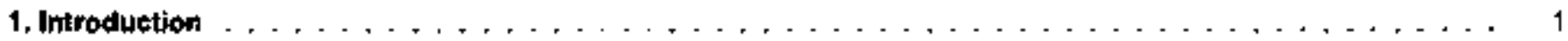

Background . . . . . . . . . . . . . . . . . . . . . . . . . 1

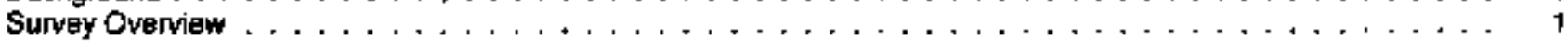

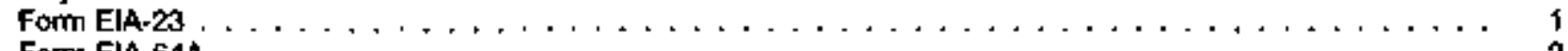

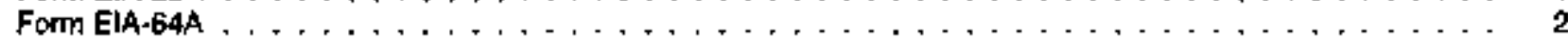

Data Collecton operations. . . . . . . . . . . . . . . . . . . 2

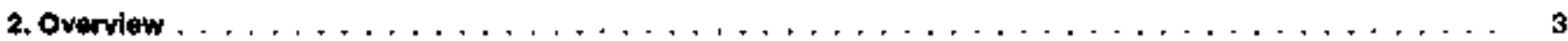

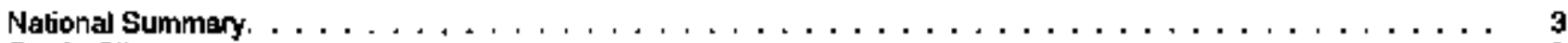

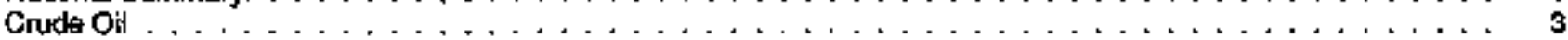

Nalural Gas . . . . . . . . . . . . . . . . . . . . . . . . . . . 3

Natural Gas Liquids . . . . . . . . . . . . . . . . . . . . . . . . . .

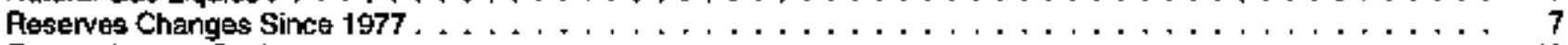

Economics and Drilling . . . . . . . . . . . . . . . . . . . . . . 10

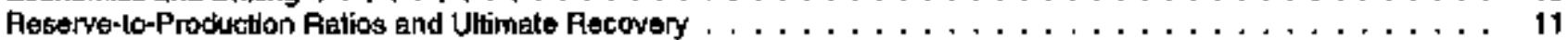

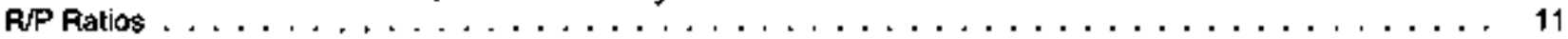

Proved Ultimate Recovery . . . . . . . . . . . . . . . . . . . . . . . . . 14

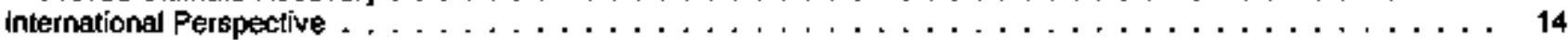

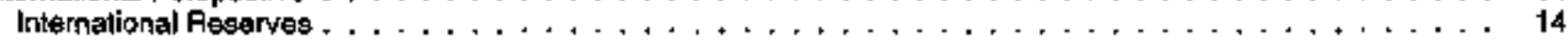

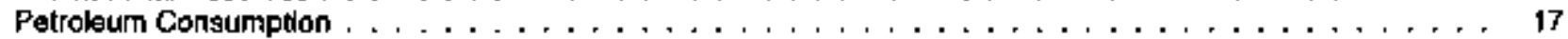

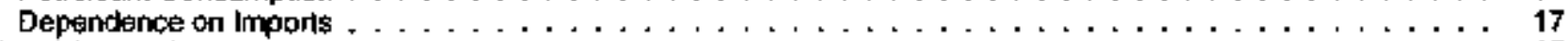

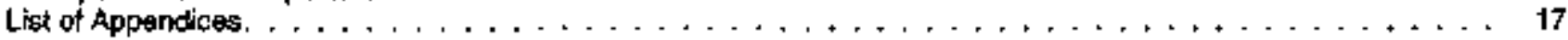

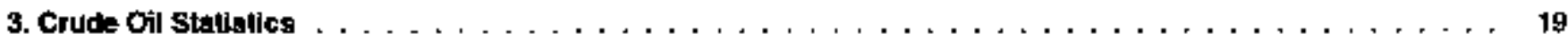

Proved Reserves . . . . . . . . . . . . . . . . . . . . . . . 19

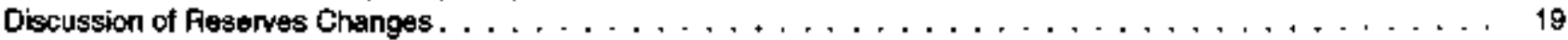

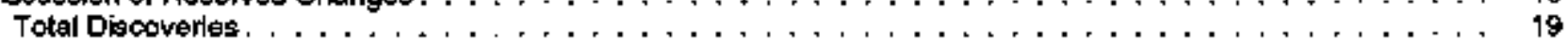

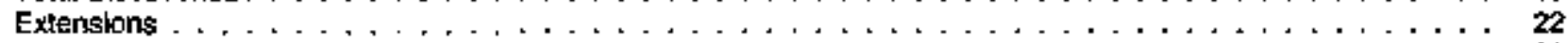

New Fiekt Discoveries . . . . . . . . . . . . . . . . . . . . . . .

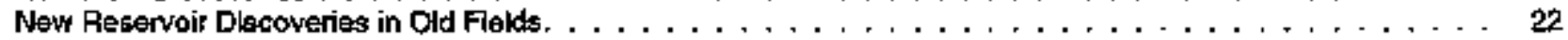

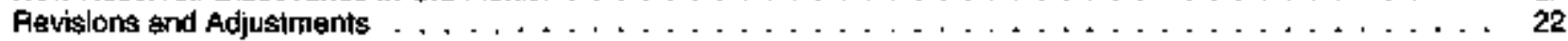

Production . . . . . . . . . . . . . . . . . . . .

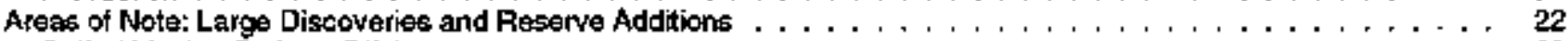

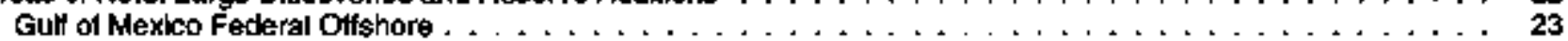

Califomia . . . . . . . . . . . . . . . . . . . . 23

Texas. . . . . . . . . . . . . . . . . . . . . . 24

Alask . . . . . . . . . . . . . . . . . . . . . . 24

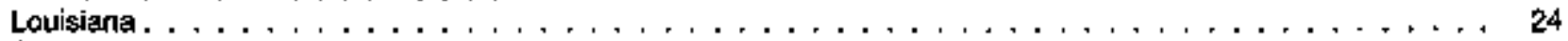

Other Gain Areas. . . . . . . . . . . . . . . . . . . . . . . . . . 24

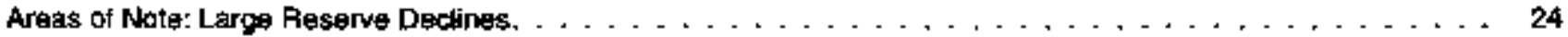

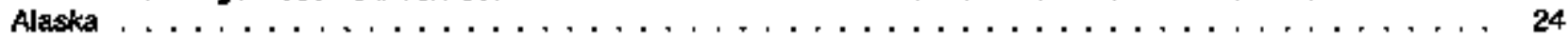

Texas. . . . . . . . . . . . . . . . . . . . . 24

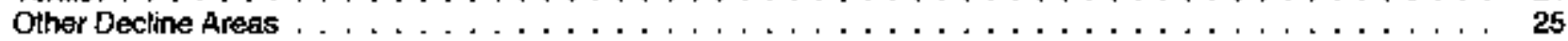

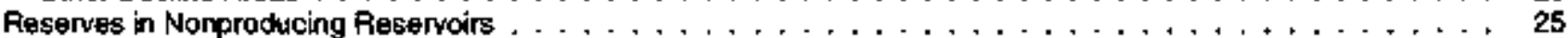

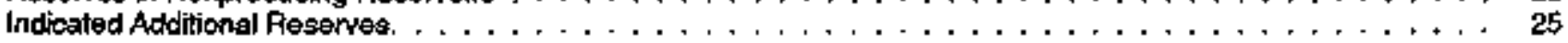

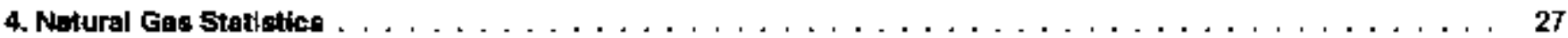

Dry Natural Gas . . . . . . . . . . . . . . . . . . . . . . . . . . 27

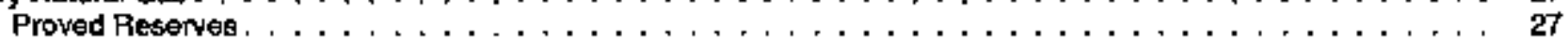

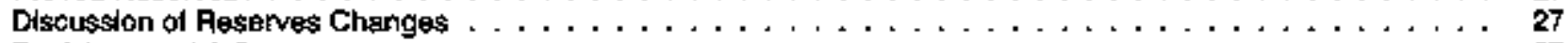

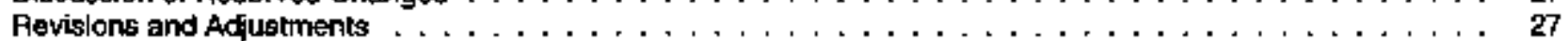

Discoveries. . . . . . . . . . . . . . . . . . . . . . . 30

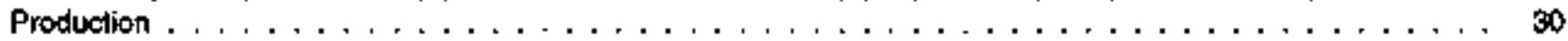

Wet Natural Gas. . . . . . . . . . . . . . . . . . . . . . . . . . .

Nonassociated Natural Ges. . . . . . . . . . . . . . . . . . . . . . . . . . . . . . . . . 30

Proved Reserves. . . . . . . . . . . . . . . . . . . . .

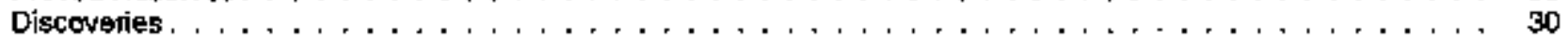




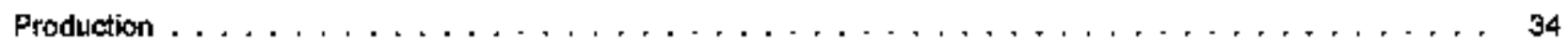

Associated-Dissolved Natural Gas . . . . . . . . . . . . . . . . . . . . . . . 34

Proved Reserves. . . . . . . . . . . . . . . . . . . . . . . . . . . . . .

Production $\ldots \ldots \ldots \ldots \ldots \ldots$

Coabed Methane . . . . . . . . . . . . . . . . . . . . . . . . . . . 34

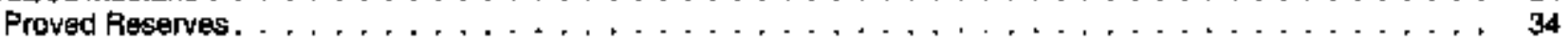

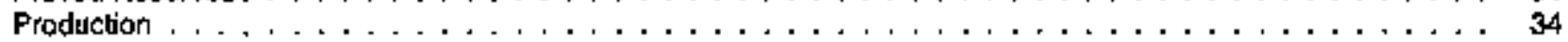

Areas of Note: Large Discoveries and Reserves Additions . . . . . . . . . . . . . . . . . . . . . . . . . 35

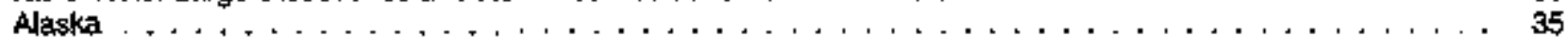

Gull of Wexico . . . . . . . . . . . . . . . . . . . . . . . . . . 35

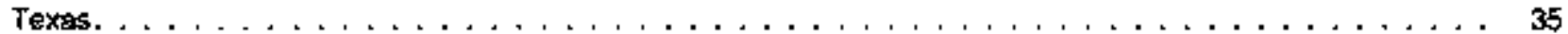

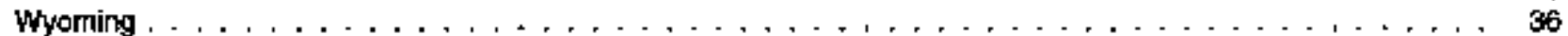

Areas of Note: Large Reserves Declines . . . . . . . . . . . . . . . . . . . . . . $\$ 6$

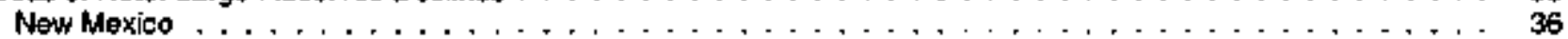

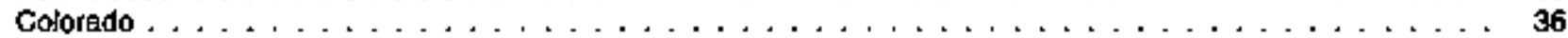

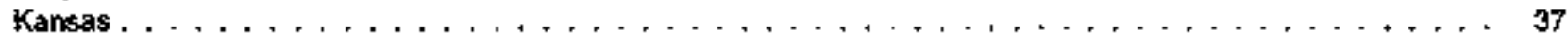

Reserves in Nonproctucing Reservoirs . . . . . . . . . . . . . . . . . . . . . . 3 T

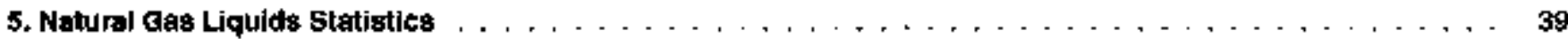

Nalural Gas Llquids . . . . . . . . . . . . . . . . . . . . . . . . . . . . 39

Proved Peserves . . . . . . . . . . . . . . . . . . . . . . . . . . 39

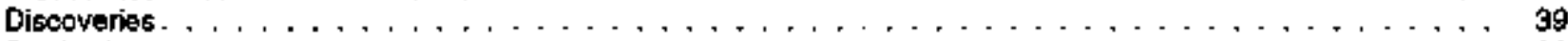

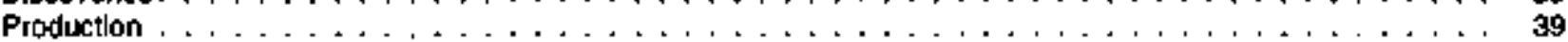

Nalural Gas Plant Eiquids. . . . . . . . . . . . . . . . . . . . . . . . . 39

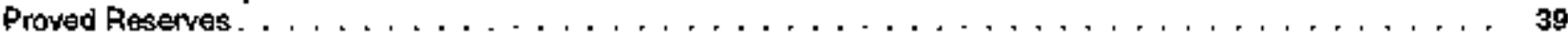

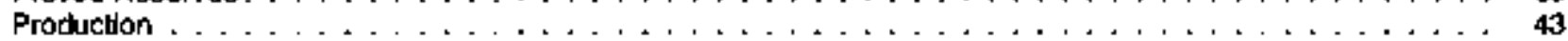

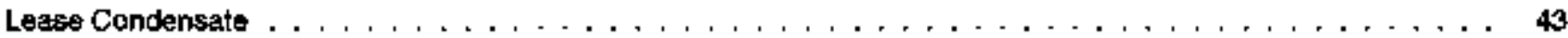

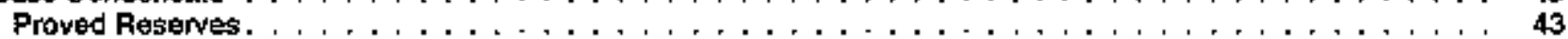

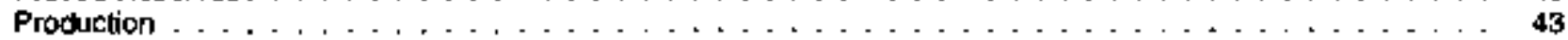

Heserves in Nonproducing Reservoirs . . . . . . . . . . . . . . . . . . . 43

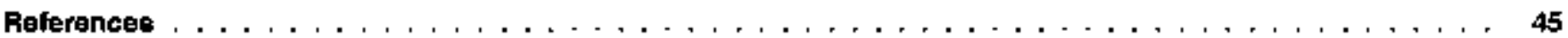

Appendices

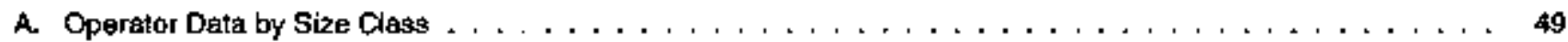

B. Top 100 Oil and Gas Fields for $1997 \ldots \ldots \ldots$

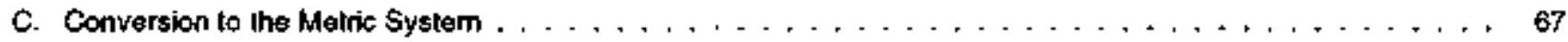

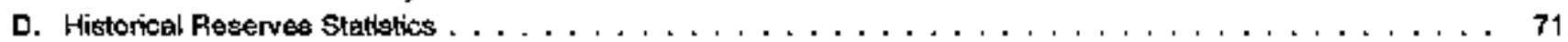

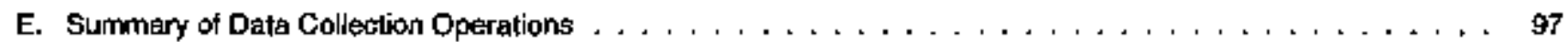

F. Statisticgl Consideralions . . . . . . . . . . . . . . . . . . . . . . . . . 10s

G. Estimation of Feserves and Fesources. . . . . . . . . . . . . . . . . . . . . . . . 125

H. Maps of Selected State Subdivisions . . . . . . . . . . . . . . . . . . . . . . 135

1. Annual Survey Forms for Domestic Oil and Gas Reserves . . . . . . . . . . . . . . . . . . . . . . . . . . . . 145

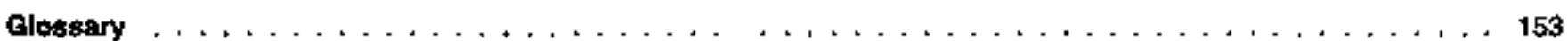

\section{Tables}

1. U.S. Prpwed Reservas of Crude Oh, Dry Natural Gas and Nahural Gas Liquids, 1987-1997. . . . . . . . . . . 4

2. Peserves Changes, $1977-1997 \ldots \ldots \ldots \ldots$

3. U.S. Average Annual Domestic Welihead Prices for Crude Oil and Natural Gas, and the
Average Number of Active Rotary Drilling Figs, 1970-1997 . . . . . . . . . . . . . . . . 10

4. U.S. Exploratory and Devolopment Wenl Completionis, 1970-1997 . . . . . . . . . . . . . . . . . . . 12

5. International Oil and Nelural Gas Reserves as of Dacember 31, 1997 . . . . . . . . . . . . . . . . . . . . . 16

6. Crude Oil Proved Feserves, Reserves Changes, and Production, 1997 . . . . . . . . . . . . . . . . . . . . 20

7. Repolted Indleated Additlonal Grude Oil Reserves, 1997 . . . . . . . . . . . . . . . . . . . . . . . . . 26

8. Dry Natural Gas Proved Reserves, Reserves Changes, and Production, 1997 . . . . . . . . . . . . 28 
9. Natural Gas Proved Reserves, Reserves Changes, and Production.

Wẹt Alter Lease \$eperation, $1997 \ldots \ldots \ldots \ldots$

10. Nonassociated Natura| Gas Prowed Reserves, Reservas Changes, and Production,

Wet After Lease Separation, $1997 \ldots \ldots \ldots$

11. Associated-Dissolved Naltral Gas Proved Reserves, Reserves Changes, and Production,

Wet After Lease Separation, $1997 \ldots \ldots \ldots$

12. U.S. Coalbed Methane Proved Reserves and Production, 1993-1997 . . . . . . . . . . . . . . . . . . . 35

13. Reported Reserves of Natural Gas, Wẹt Attẹr Lease Separation, in Nonproducing Resenuoirs, 1997 . . . . . . . 38

14. Natural Gas Liquids Proved Reserves, Reserves Changes, and Production, 1997 . . . . . . . . . . . 40

15. Natural Ges Plant Liquids Proved Reserves and Production, 1997 . . . . . . . . . . . . . . . . 41

16. Lease Condensate Proved Reserves and Production, 1997 . . . . . . . . . . . . . . . . . . . . . . . . 42

\section{Figures}

1. U.S. Crude Oil Proved Resenves, 1986-1997 . . . . . . . . . . . . . . . . . . . . . . . . 5

2. Components of Reserves Changes for Crude Oil, 1986-1997 , . . . . . . . . . . . . . . . 5

3. U.S. Cry Nalural Gas Proved Reserves, 1986-1997 . . . . . . . . . . . . . . . . . . . . . . . . . . 6

4. Components of Feserves Changes for Dry Nahural Gas, 1986-1997 . . . . . . . . . . . . . . . . 6

5. U.S. Natural Gas Liquids Proved Feserves, 1986-1997 , . . . . . . . . . . . . . . . . . . . . . B

6. Components of Reserves Changes for Nalura Gas Liqulds, 1986-1997 . . . . . . . . . . . . . . . . . . . . . . . 8

7. U.S. Exploratory Gas Well Completions, 1977-1997 . . . . . . . . . . . . . . . . . . . . . . . 13

8. U.S. Exploratory Oil Well Completions, 1977-1997 . . . . . . . . . . . . . . . . . . . 13

9. U.S. Tolat Discoveries of Dry Natural Gas per Exploralony Gas Wel Completion, 1977-1997 . . . . . . . . . . . 13

10. U.S. Totel Discovertes of Crude Oll per Exploratory Oil Well Gompletion, 1977-1997 . . . . . . . . . . . . . . . 13

11. Resenes-10-Production Ratios tor Crude OA, 1945-1997. . . . . . . . . . . . . . . . . . . . . . 15

12. Reserves-10-Production Ratios tor Wet Natural Gass, 1945-1997 . . . . . . . . . . . . . . . . . . . . . 15

13. Components of Uitmate Recovery for Crude Oil and Lease Condensade, 1977-1997 . . . . . . . . . . . . . . . . 15

14. Components of Ultimate Pecovery for Wet Natural Gas, 1977-1997 . . . . . . . . . . . . . . . . . . . . 15

15 Reserve Addilions Nearly Replace U.S. Oil Production in 1997 . . . . . . . . . . . . . . . . . . . . . . . . 19

16. 1997 Crude Oil Proved Reserves by Area ... . . . . . . . . . . . . . . . . . . . . . . . 21

17. Changes in Crude Oi Proved Reserves by Area, 1998 to $1997 \ldots \ldots$. . . . . . . . . . . . . . . .

18. Feserve Addilions Exceed U.S. Natural Gas Production 3 Years in a Rour . . . . . . . . . . . . . . . . . . 27

19. 1997 Dry Natural Gas Proved Reserves by Area . . . . . . . . . . . . . . . . . . . . . . . . . 29

20. Changes in Dry Natural Gas Proved Reserves by Area, 1996 to 1997 . . . . . . . . . . . . . . . . . . . . 29

21. Coatbed Wethane Proved Regerves, 1990-1997 . . . . . . . . . . . . . . . . . . . . . 34 



\section{Executive Summary}

U.S. proved reserves of crude oil increased in 1997 for the first time in a decade. Natural gas reserves were up for the fourth year in a row. Sturprisingly lange revisions in some of California's old and heavy oil fields provided over half the oil increase. Oil and gas discoveries in the Federal offshore-several in deep water-also played a major role in the oil reserves increase and the continuation of gas reserves increases. Successful oil and gas well completions were up.

\begin{tabular}{|lr|}
\hline As of December 31, proved reserves were: \\
\hline \multirow{2}{*}{ Dry Natural Gas (billion cubic feet) } \\
1996 & 166,474 \\
1997 & 167,223 \\
Increase & $+0.4 \%$ \\
Crude Oil (million barrels) & \\
1996 & 22,017 \\
1997 & 22,546 \\
Increase & $+2.4 \%$ \\
Natural Gas Liquids (million barrels) & \\
1996 & 7,823 \\
1997 & 7,973 \\
Increase & $+1.9 \%$ \\
& \\
\hline
\end{tabular}

Proved reserves are those quantities that geological and engineering data demonstrate with reasonable certainty to be recoverable in future years from known reservoirs under existing economic and operating conditions. Petroleum engineering and geological judgment are required in estimating proved reserves; therefore, the results are not precise measurements. This report of 1997 U.S. proved reserves of crude oil, natural gas, and natural gas liquids is the 21st in the annual series prepaned by the Energy Information Administration.

\section{Natural Gas}

Gas reserve additions replaced gas production again in 1997. Lower 4B States natural gas reserves declined slightly in 1997, but increases in Alaskan reserves due to revisions and adjustments more than made up for it. Gas production has trended up since 1986 and is now 3.6 trillion cubic feet higher, yet, in each of the last four years neserve additions replaced production. The largest gas reserve additions were in the Federal Offshore closely followed by those in
Texas. For 1997, revisions and adjustments to merves in existing fields were the lowest in the past decade and half of 1996's. In contrast, total gas discoveries were the highest in the past decade.

U.S. total discoveries of dry gas reserves were 15,648 billion cubic feet in 1997, up 27 percent from a very good 1996. Texas and the Gulf of Mexico Federal Offshore accounted for over three-fifths of them. Total discoveries, which equaled 81 percent of 1997 gas production, are those reserves attributable to field extensionts, new field discoveries, and netw reservoir discoperies in old fields. They result from drilling exploratory wells.

- New field discoveries were 2,681 billion cubic feet, up substantially from 1996 and twice the prior 10-year average.

- Field extensions were 10,585 billion cubic feet, up from 1996 and 68 percent above the prior 10-year average.

- Neto reservoir discoteries in old fields were 2,382 billion cubic feet, down from 1996 .

Coalbed methane reserves continued to grow faster than conventional natural gas reserves, accounting for nearly 7 percent of $1997^{\prime} \mathrm{s}$ proved gas reserves. Coalbed methane production, now over 5 percent of the U.S. total, also increased faster.

Other 1997 natural gas events of note:

- Exploratory gas well completions were down slightly at 538 in 1997.

- Total discoveries per exploratory gas well were up in 1997 and more than four times the rates of the early 1980s.

- Natural gas prices at the wellhead increased slightly to an annual average of $\$ 2.23$ per thousand cubic feet.

\section{Crude Oil}

Crude oil reserve additions replaced 125 percent of 1997 oil production, a result of the first reșerves increase in a decade.

Total discoveries of crude oil were 1,233 million barrels in 1997, roughly twice as high as the prior 10-year average and a third higher than in 1996. The Gulf of Mexico Federal Offshore accounted for over 
50 percent, much of it in deep water, Texas had 15 percent, and Alaska 11 percent.

Improved technologies for exploration and deepwater production enhanced the ability to discover and develop offshore fields, especially in deep water. British Borneo's Morpeth project in 1700 feet of water is an example of the use of state-of-the-art engineering and technology that lowers field development cost in deep water. The field needed a way to inject water to maintain reservoir pressure and oil production. A mini-tension leg platform (SeaStar) and the first stubsea injection well in the Gulf of Mexico were used to develop this oil field that would otherwise have ben uneconomic.

New field discoveries were 637 million barrels, more than twice those of 1996 and over 5 times the prior 10-year average. The Gulf of Mexico Federal Offshore had 79 percent of the new field discoveries, Alaska had 18 percent, and the rest of the country had less than 3 percent. Well over half of the proved reserves of oil in the Gulf of Mexico are now in deep water (water depths greater than 200 meters, about two football fields).

Reserves in new fields are not counted by ELA until they are proved. Many years may elapse between the time an exploratory well locates a new field and the year those oil or gas reserves are accepted as proved. For example, the well that discovered the West Sak Field in Alaska was drilled in 1971. But it was not until 1997 that production facility sharing arrangements and new low-cost drilling and completion technologies made the field economic io develop and hence proved.

- New reservoir discoveries in old fields were 119 million barrels, down some from 1996 but still close to the prior 10-year average.

- Fiztd extersions, up in 1997, added 477 million barrels of proved oil reserves.

- Revisions and adjuslments were 1,434 million barrels in 1997. Even in an outstanding discovery year, they still accounted for over half of total oil reserve additions. Texas and California, States with large oil reserves and large enhanced oil recovery projects, had over half of revistons and adjustments.

Technology improvements allowed California operators to revise upward the reserves in old fielcs with viscous heavy oil. For example, more oil is efficiently heated by replacing less efficient steam cycling processes with continuous steam injection that lowers the oil viscosity allowing it to move more easily to producing wells. This lowers costs and increases production rates.

Alaska's reserves declined in 1997 despite substantial new field discoveries because there were not enough revisions and adjustments to replace production.

Other 1997 crude oil events of note:

- The anmual average domestic first purchase price for crude oil declined 7 percent during 1997 to $\$ 17.24$ per barrel.

- Exploratory oil completions were down some at 418. However, total oil well completions increased to 10,310 owing to an increase in oil development activity.

- Total discoverjes per exploratory oil well were up over 50 percent in 1997 , and over six times the rates of the early 1980 's when oil prices and the dritling boom peaked.

Indicated additional reserves of crude oil increased to 3,207 million barkels in 1997. These are conde oil volumes that may become economically recoverable from known reservoirs through the application of improved recovery techniques using current technology. The presence of large indicated additional reșerves in north Alaska, California. Texas, and Louisiana implies that significant upward revisions to crude oil proved reserves can continue to occur in the future.

\section{Natural Gas Liqulds}

U.S. natural gas liquids proved reserves increased 2 percent to 7,973 million barrels in 1997. Natural gas liquids reserves are the sum of natural gas plant liquids and lease condensate reserves.

Total proved reserves of liquid hydrocarbons (crude oil plus natural gas liquids) were $30,525 \mathrm{million}$ barrels in 1997, another increase. Natural gas liquids were about a quarter of total proved reserves of liquid hydrocarbon in $\mathbf{1 9 9 7 .}$

\section{Data}

These estimates are based upon analysis of data from Form ELA-23, "Annual Survey of Dornestic Oil and Gas Reserves," filed by 3480 operators of oil and gas wells, and Form EIA-64A, "Annulal Report of the Origin of Natural Gas Liquids Production," filed by operators of 697 active natural gas processing plants. The U.S. proved reserves estimates for crude oil and natural gas are associated with sampling error's of less than 1 percent. 


\section{Introduction}

\section{Background}

The principal focus of this report is to provide accurate annual estimates of U.S. proved reserves of crude oil, natural gas, and natural gas liquids. These estimates are essential to the development, implementation, and evaluation of national energy policy and legislation. In the past, the Government and the public relied upon industry estimates of proved reserves. However, the industry ceased publication of reserve estimates after its 1979 report.

In response to a recognized need for credible annual proved reserves estimates, Congress, in 1977, required the Department of Energy to prepare such estimates. To meet this requirement, the Energy Information Administration (EiA) developed a program that established a unified, verifiable, compreherisive, and continuing aruual statistical series for proved reserves of crude oil and natural gas. It was expanded to include proved reserves of natural gas liquids for the 1979 and subsequent reports.

\section{Survey Overview}

EIA defines proved reserves, the major topic of this report, as those volumes of oil and gas that geologica] and engineering data demonstrate with reasonable certainty to be recoverable in future years from known reservoirs under existing economic and operating conditions. There are other categories of reserves, but by definition they are more speculative and less precise than proved reserves. Readers who are unfamiliar with the distinctions between types of reserves or with how reserves fit in the description of overall oil and gas resources should see Appendix $G$.

While the primary topic of this report is proved reserves, information is also presented on indicaled additional crude oil reserves. Indicated additional crude oil reserves are not included in proved reserves because of their uncertain economic recoverability. When economic necoverability is demonstrated, these volumes will be reclassified and transferred to the proved reserves category as positive revisions.
This report provides proved reserves estimates for calendar year 1997. It is based on data filed by large operators of oil and gas wells on Form ELA-23, "Annual Survey of Domestic Oil and Gas Reserves," and by operators of all natural gas processing plants on Form ElA-64A, "Annual Report of the Origin of Natural Gas Liquids Production." The U.S. crude oil and natural gass proved reserves estimates are associated with sampling errors of less than 1 percent at a 95-percent confidençe level.

\section{Form ElA-23}

On Form EIA-23, an operator is defined as an organization or person responsible for the management and day-to-day operation of oil and/or gas wells. This definition eliminates responses from royalty owners, working interest owners (unless they are also operators), and others not directly responsible for oil and gas production operations.

Operator size categories are based upon their annual production as indicated in various Federal, State, and commercial records. Large operators are those that produced at least 2.5 minlion barrels of crude oil or 15 billion cubic feet of natural gas, or both, during the report year. Intermediate operators produced less than large operators, but more than 400,000 barrels of crude oil or 2 billion cubic feet of natural gas, or both. Small operators are those that produced less than intermediate operators. All data are reported on a total operated basis, encompassing all proved reserves and production assocjated with wells operated by an individual operator. This concept is also called the "gross operated" or " $8 / 8$ ths" basis.

Large operators and most intermediate size operakors report reserves balance data on Form ELA-23 to show how and why reserves components changed during the year on a field-by-field basis. Intermediate size operators who do not keep reserves data were not asked to provide estimates of reserves at the beginning of the year or annual changes to proved reserves by component of change; ie., revisions, extensions, and new discoveries. These volumes were estimated by applying an algebraic allocation scheme that preserved the relative relationships between these items within each State or State subdivision, as reported by large and intermediate operators. 
The pub]ished reserve estimates include an additional term, adjustments, calculated by the ElA, that preserves an exact annual reserves balance of the form:

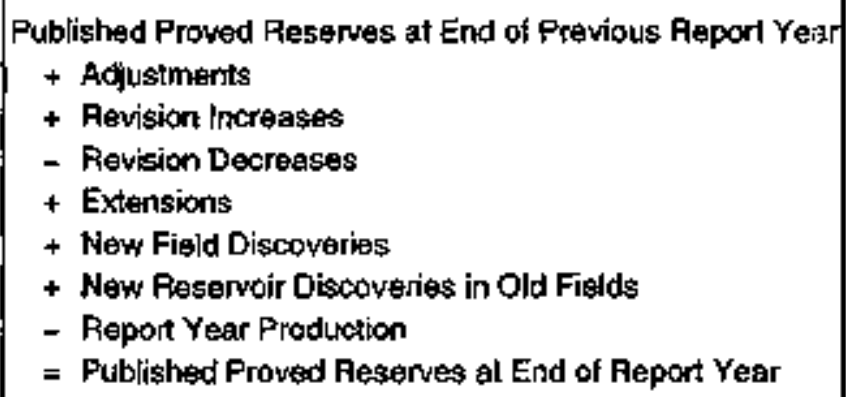

Adjustments are the arnual changes in the publisted reserve estimates that cannot be attributed to the estimates for other reserve change categories. They result from the survey and statistical estimation methods employed. For example, variations caused by changes in the operator frame, different randors samples, changes in reserve estimates following ownership changes, incorrectly reported data, er imputations for missing or urueported reserve changes can contribute to adjustments.

\section{Form ElA-64A}

Form ELA-64A data were first collected for the 1979 survey year in order to develop estimales for totzl natural gas liquids reserves. Data on liquids recoveret. from natural gas, as reported by natural gas processing plant operators, are combined with lease condensate data collected on Form EIA-23 to provide the total natural gas liquids reserves estimates.

\section{Data Collection Operations}

An intensive effort is made each year to maintain an accurate and complete survey frame consisting of operators of oil and gas wells and of natural ga.3 processing plants. The Form ElA-23 operator frame contained 22,678 probable active operators and the Form EIA-64A plant frame contained 654 probable active natural gas processing plants in the United States when the 1997 surveys were initiated. As usual, additional operators were added to the survey as it progressed, and many operators initially in the sample frame were found to be inactive in 1997.

For the report year 1997, EIA mailed 641 ElA-23 forms to all known large and intermediate size oil and gas well operators that were believed to be active during 1997. Of these, $\$ 1$ were found to be nonoperators that did not have successor operators in 1997. Data were received from 587 operators, an overall response tate of 100 percent of the active operators in the Form EIA-23 survey. ELA mailed 654 ELA-64A forms to natural gas processing plant operator's. More than one form is received for a plant that has more than one operator during the year. Forms were received from 100 percent of the operators of the 652 unique active natural gas processing plants in the Form ElA-64A survey.

National estimates of the production volumes for crude oil, lease condensate, natural gas liquids, and dry natural gas based on Form EIA-23 and Form EIA-64A were compared with corresponding official production volumes published by ElA, which are obtained trom non-survey based State sources. For report year 1997, the Form EIA-23 National production estimates were 1.8 percent lower than the comparable Petrolesm Stipply Annual 1997 volumes for crude oil and lease condensate combined, and were 1.6 percent higher than the comparable Natural Ges Amnal 1997 volume for 1997 dry natural gas. For report year 1997 , the Form EIA-64A National estimates were 4.1 percent higher than the Petroleum Stupply Anwual 1997 volume for natural gas plant liquids production. 


\section{Overview}

\section{National Summary}

The United States had the following proved reserves as of December 31, 1997:

- Crude Oij - 22,546 million barrelis

- Dry Natural Gas - 167,223 billion cubic feet

- Natural Gas Liquids - 7,973 million barrels.

This Overview summarizes the 1997 proved reserves balances of crude oil, dry natural gas, and natural gas liçuids on a National level and provides historical comparisons between 1997 and years past.

Table 1 lists the estimated annual reserve balances since 1987. In 1997, proved reserves of crude oil increased by 2.4 percent, the first increase in the last decade. Proved reserves of dry natural gas increased by 0.4 percent over the 1996 proved reserves level, making 1997 the fourth consecutive year that gas reserves have increased.

\section{Crude Oil}

Proved reserves of crude oil increased by 529 million barrels in 1997. The largest increase occurred in the Federal Offshore portion of the Gulf of Mexico, where 382 million barrels of crude oil proved reserves were added, California was a close second, where reserves additions in the heavy oil fields raised the State total by 313 mithion barrels. Figure 1 shows the crude oil proved reserves levels by major region and Figure 2 shows the components of reserves changes from 1987 through 1997 .

As shown in Figure 2, total reserve additions (the positive side of the scale) were up substantially in 1997 . due to large increases in the volumes of new field discoveries and revisions and adjusiments. Production of crude oil (the negative side of Figure 2's scale) declined slightly for the sixth year in a row.

Tolal discaveries are those reserves attributable to field extensions, new field discoperies, and new reservoir discoveries in old fields. There were 1,233 million barrels of total discoweries of crude oil proved reserves in 1997. This is 33 percent more than in 1996.

Extensions added 477 million barrels of proved reserves. This is 12 percent less than in 1996 (543 million barrels) and 9 percent more than the average extensions in the ptior 10 years ( 436 million barrels).

New freld discoteries were 637 million barrels, more than twice the 1996 level and more than five times the average volume discovered in the prior 10 years $(122$ million barrels). New field discoveries in Alaska and the Gulf of Mexico Federal Offshore made up 98 percent of the 1997 volume.

New reserwoir discoveries in old fields added 119 million barrels of proved reserves. This is less than the 19\% level (14l millian barrels) and the prior 10-year average for the United States (135 million barrels).

Revisions ath adjustments added 1,434 million barrels of proved reserves. This is 57 percent more than the 1996 volume (912 million barrels) and 11 percent more than the average volume in the prior 10 years $(1,293$ million barrels).

Production removed an estimated 2,138 million barreis of proved resserves from the National total. Production was down 2 percent from 1996's level $\{2,173$ million barrels) and at 86 percent of the prior 10-year average (2,473 million barrels $\}$. Reserves additions in 1997 replaced 125 percent of production.

\section{Natural Gas}

U.S. proved reserves of dry natural gas increased for the fouth year in a row in 1997-up 0.4 percent from 1996 's level to a total of 167,223 billion cubic feet. Dry natural gas reserves declined by $\mathbf{5 1 9}$ billion cubic feet in the Lower 48 States, but reserves additions in Alaska more than made up for that. Figure 3 shows the dry natural gas proved reserves levels by major region and Figure 4 shows the components of reserves changes from 1987 through 1997. Dry natural gas production increased for the second consecutive year in 1997, and operators were able to replace 104 percent of 1997 's dry gas production with new reserves.

For 1997, US. toial discoveries of dry gas neserves were 15,648 billion cubic feet, up 27 percent from 1996. Tolal discootries were 78 percent of all reserve additions in 1997, while revisions and adjustments provided the remaining 22 percent. 
Table 1. U.S. Proved Reserves of Crude Oil, Dry Natural Gas, and Matural Gas Liquids, 1967-1997

\begin{tabular}{|c|c|c|c|c|c|c|c|c|c|c|c|}
\hline rant & Ad]unimwints & 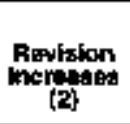 & $\begin{array}{c}\text { Revizion } \\
\text { Deoreateat } \\
\text { (3) }\end{array}$ & $\begin{array}{l}\text { Rewtsigns" } \\
\text { adjugiment: } \\
\text { (4) }\end{array}$ & Extanglons: & 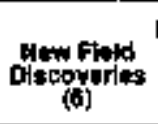 & 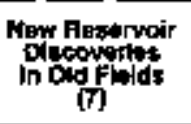 & Dleoterwestes & $\begin{array}{l}\text { Proctuction } \\
\text { (p) }\end{array}$ & 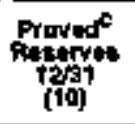 & $\begin{array}{l}\text { Changes } \\
\text { trom } \\
\text { Prlor Yoar } \\
\text { (11) }\end{array}$ \\
\hline & \multicolumn{11}{|c|}{ Crudo Oil \{nillion barrels of 42 U.S. gallons\} } \\
\hline 199 & 233 & 3,997 & 1,371 & 2,549 & 484 & $\theta 6$ & 111 & 681 & 2.873 & 27,256 & +367 \\
\hline 1969 & 364 & $2,68 \mathrm{~A}$ & 1,221 & 1,827 & 355 & 71 & 127 & 553 & 2,811 & 26,825 & 431 \\
\hline 190 & 213 & 2.698 & 1,385 & 1,546 & 514 & 112 & 90 & 716 & 2,596 & 26,501 & -304 \\
\hline 1990 & 80 & 2,483 & 1,600 & 1,569 & 458 & 9 & 135 & 689 & 2,505 & 28,254 & .247 \\
\hline 1981 & 163 & 2,097 & 1,674 & 386 & 305 & 97 & 52 & 554 & 2,512 & 24,682 & $-1,972$ \\
\hline 1992 & 290 & 1,百的 & 1,069 & 1.025 & $39 t$ & $B$ & 85 & $4 \mathrm{Bd}$ & 2,446 & 29,745 & -937 \\
\hline 1993 & 271 & 2.011 & 1,518 & 788 & s6B & $\$ 10$ & 110 & 786 & 2,339 & 22.95 & -788 \\
\hline 1994 & 189 & 2,384 & 1,357 & 1,196 & 397 & 64 & 111 & 572 & 2,268 & 22,457 & .500 \\
\hline 2905 & 122 & 1,823 & 795 & 1,150 & 500 & 114 & 343 & 957 & 2,213 & 22,351 & -106 \\
\hline $19 * 3$ & 175 & 1,723 & 996 & 912 & 643 & 243 & 141 & $9 z 7$ & 2,173 & 22,017 & -334 \\
\hline 1997 & 520 & 1,990 & $1, \cos 4$ & 1,434 & $4 \pi$ & 837 & 119 & $1,23.9$ & 2,138 & 22,548 & +529 \\
\hline
\end{tabular}

Dry Maturad Gas (bilion cubic feet, 14.73 psia, $60^{5}$ Fahrenheit)

\begin{tabular}{|c|c|c|c|c|c|c|c|c|c|c|c|}
\hline 1987 & $\uparrow, 268$ & 17,527 & 14,231 & 4,564 & 4,597 & 1,0689 & 1,499 & 7.175 & 18,114 & 187.211 & 4,375 \\
\hline 1983 & 2,193 & $2 \pi, 3 \times 7$ & $d_{39,427}$ & $-12, \infty 67$ & 6,803 & 1,639 & 1.969 & 10,330 & 16,670 & $d_{169,024}$ & $-19,187$ \\
\hline 1999 & 3013 & 26.673 & 23,643 & 6,043 & 6,339 & 1,450 & 2,243 & 10,032 & 16,283 & 167,116 & -900 \\
\hline 180 & 1,557 & 18,981 & 13,443 & 7,095 & 7,052 & 2,004 & $2.4+2$ & 12,368 & 17.233 & 169,340 & $+2,2300$ \\
\hline $1 \$ 91$ & 2,960 & 19,680 & 15,474 & 7,376 & 5,000 & 848 & 1.604 & 7.542 & 17,202 & 187,062 & $-2,284$ \\
\hline 1998 & 2,234 & 18,055 & 11,982 & $\mathrm{~B}, 32 \mathrm{~B}$ & 4,675 & 649 & 1,724 & 7,048 & $17 . \$ 23$ & 165,015 & $-2,047$ \\
\hline 1983 & 672 & 17 ,597 & 12,248 & 6,321 & 6,103 & 650 & $1, \theta \in 6$ & 8,850 & 17,709 & 1ed, 415 & $-2,600$ \\
\hline 1994 & 1,945 & $\mathbf{2 1 , 3 0 5}$ & 15,981 & 7,429 & 6,941 & 1,894 & 3,480 & 12,315 & 18,322 & $1 \mathrm{~g}, \mathrm{~g}, \mathrm{~g}$ & $+1,42$ \\
\hline 1995 & 580 & 20,465 & 12,731 & $B_{, 314}$ & $6, \$ 43$ & $1, \psi_{66}$ & 2,458 & 10,961 & 17.969 & 165,148 & $+1,309$ \\
\hline 1䗆苗 & 3,795 & 17,132 & 13,046 & 7,871 & 3.757 & 1,451 & 3,710 & 12,318 & 1B,861 & 165,474 & $+1,328$ \\
\hline 1997 & -590 & 21,658 & 16,755 & 4,312 & $+0,583$ & 2,681 & 2,382 & 15,648 & 19,211 & 167,223 & +749 \\
\hline
\end{tabular}

Malural Eas Liqulus (million barrels o1 42 U.S. gallons)

\begin{tabular}{|c|c|c|c|c|c|c|c|c|c|c|c|}
\hline 1997 & $23 t$ & 847 & 658 & 122 & 213 & 39 & 55 & 307 & 747 & 9,147 & -19 \\
\hline 1988 & 11 & 1.168 & 715 & 464 & 288 & 41 & 72 & 381 & 754 & 9,238 & +61 \\
\hline 1999 & -277 & 1,143 & 1.020 & -154 & 259 & $\mathrm{~B} 3$ & 74 & 416 & 731 & 7,769 & -469 \\
\hline 1990 & -83 & 827 & 806 & 138 & 299 & 39 & 73 & 411 & 732 & 7,596 & -183 \\
\hline 1991 & 233 & 825 & 695 & 363 & 189 & 25 & 55 & 260 & 754 & 7,464 & -122 \\
\hline 1992 & 225 & 808 & $\$ 45$ & 486 & teo & 20 & 64 & 274 & $7>3$ & 7,451 & -13 \\
\hline t903 & 102 & 764 & 640 & 228 & 245 & 24 & 64 & 393 & 78 & 7,222 & 239 \\
\hline 1994 & 43 & 873 & B76 & 240 & 314 & 54 & 131 & 498 & 791 & 7,170 & -52 \\
\hline 1995 & 192 & 968 & 691 & 469 & 432 & 52 & 67 & 551 & 791 & 7,390 & +279 \\
\hline 1906 & $\$ 74$ & B44 & 660 & 649 & $4 \$ 1$ & 的 & 100 & 626 & 850 & 7, acs & +424 \\
\hline 1997 & -14 & 1.190 & 910 & 275 & $\$ 35$ & 114 & 90 & 739 & 804 & 7,973 & +150 \\
\hline
\end{tabular}

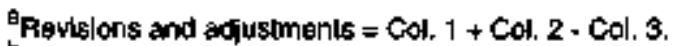

bTotal dlscoveries $=$ Col. $5+$ Col. $6+$ Coll. 7 .

EProved reserves = Col. 10 from prior year + Col. $4+$ Col. 8 - Cat. 9.

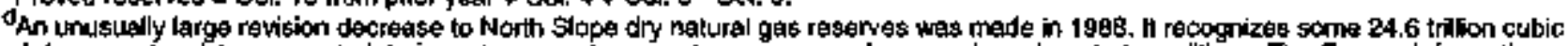
feel of downward rewistons reported duing prior years by operalors jecause of exomombe gnd mankel conditons. The Energy information Administration (ELA) in previous years caried these reserves in the proved category.

Notes: Old means discovered by a pricr year. New means dilscovered duling the raport year. The producllon estimales in this table are besed on data reported on Form ElA-23, "Annudi Survey of Domestic OH and Gas Peserves" and Form ElA-64A, "Annual Peporl of the Origin ol Natural Gas Liquikts Production." Thay may differ from the oficial Eld production dela for crude oil, naturgl gas, and natural pas fipuids for

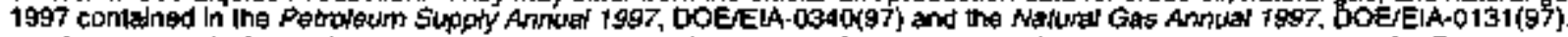

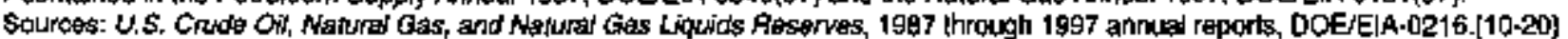


Figure 1. U.S. Crude Oil Proved Reserves, 1987-1997

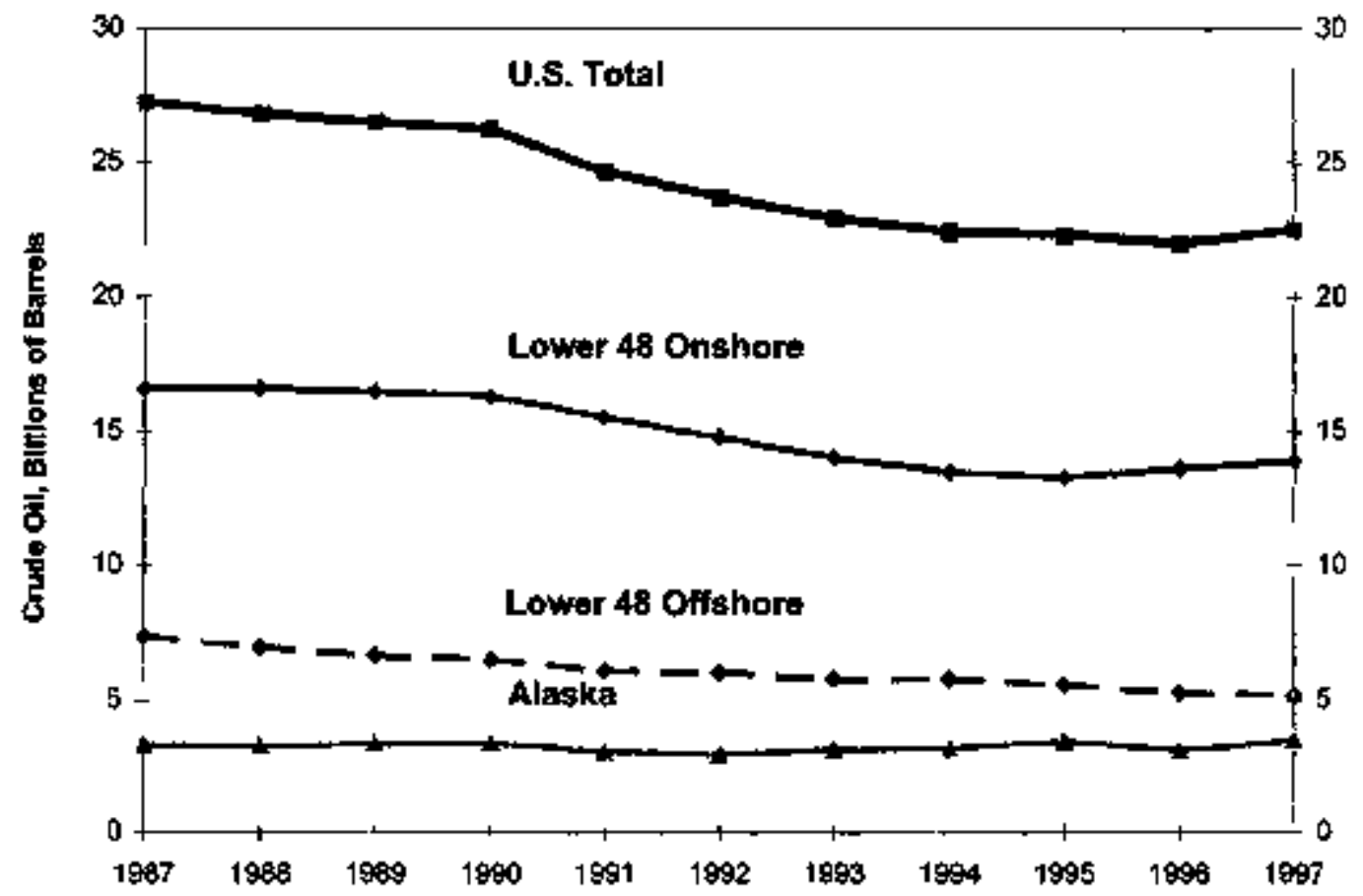

Flgure 2. Components of Reserves Changes for Crude Oil, 1987-1997

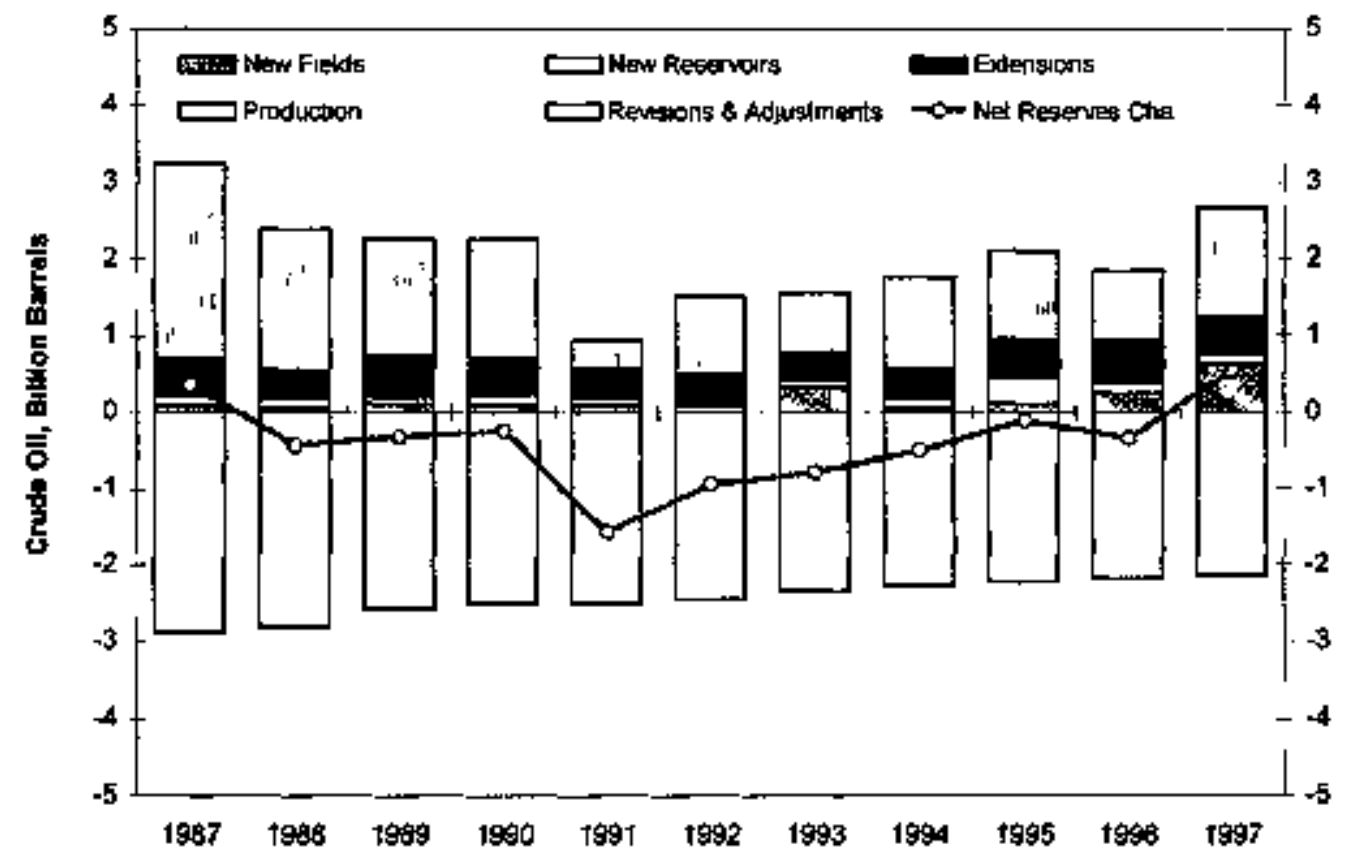

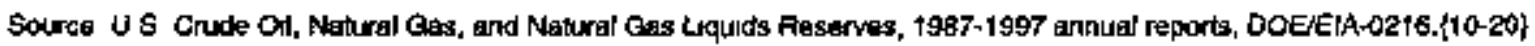


Figure 3. U.S. Dry Naturat Qas Proved Reserves, 1987-1997

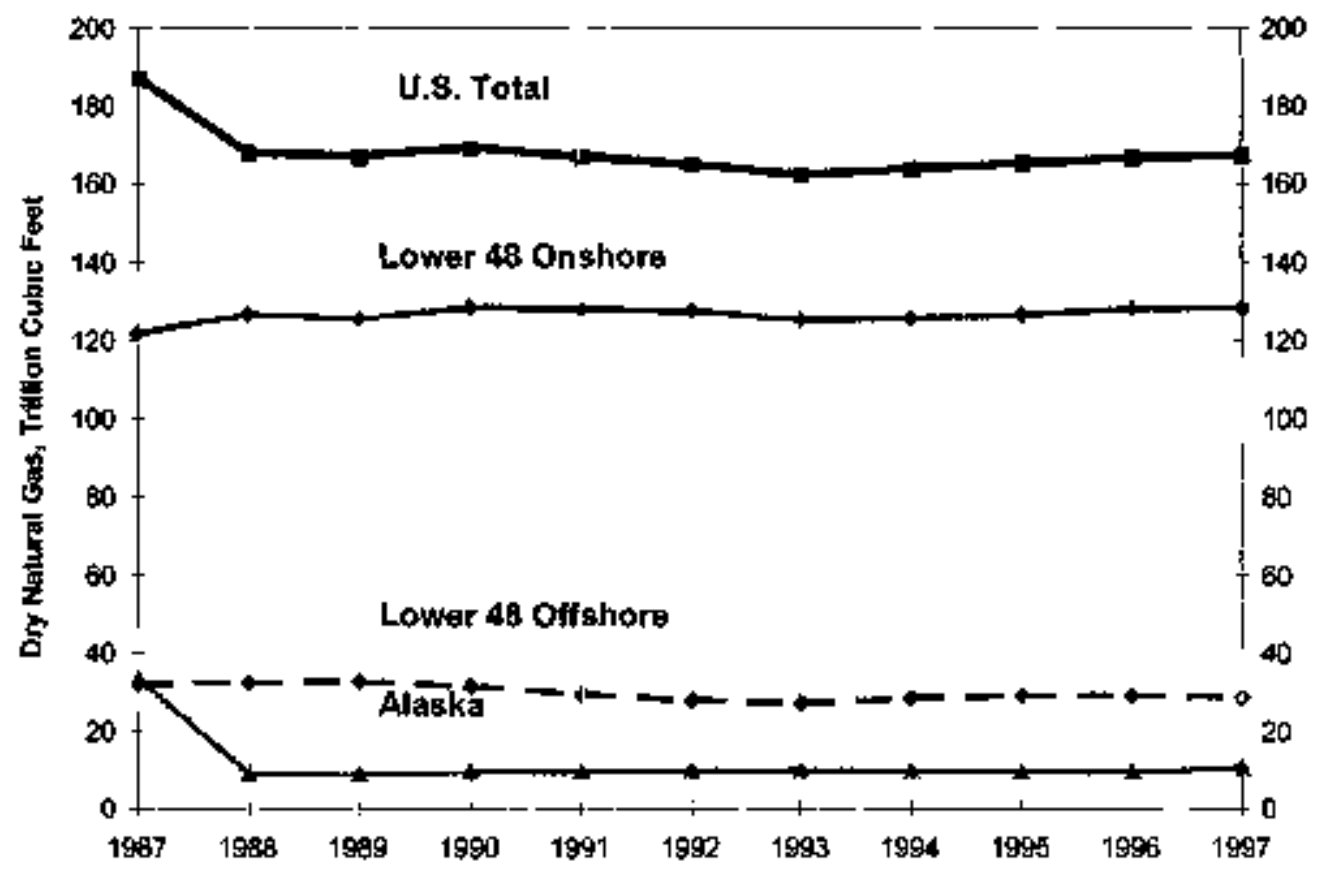

Figure 4. Components of Peserves Changes for Dry Natural Gas, 1987-1997

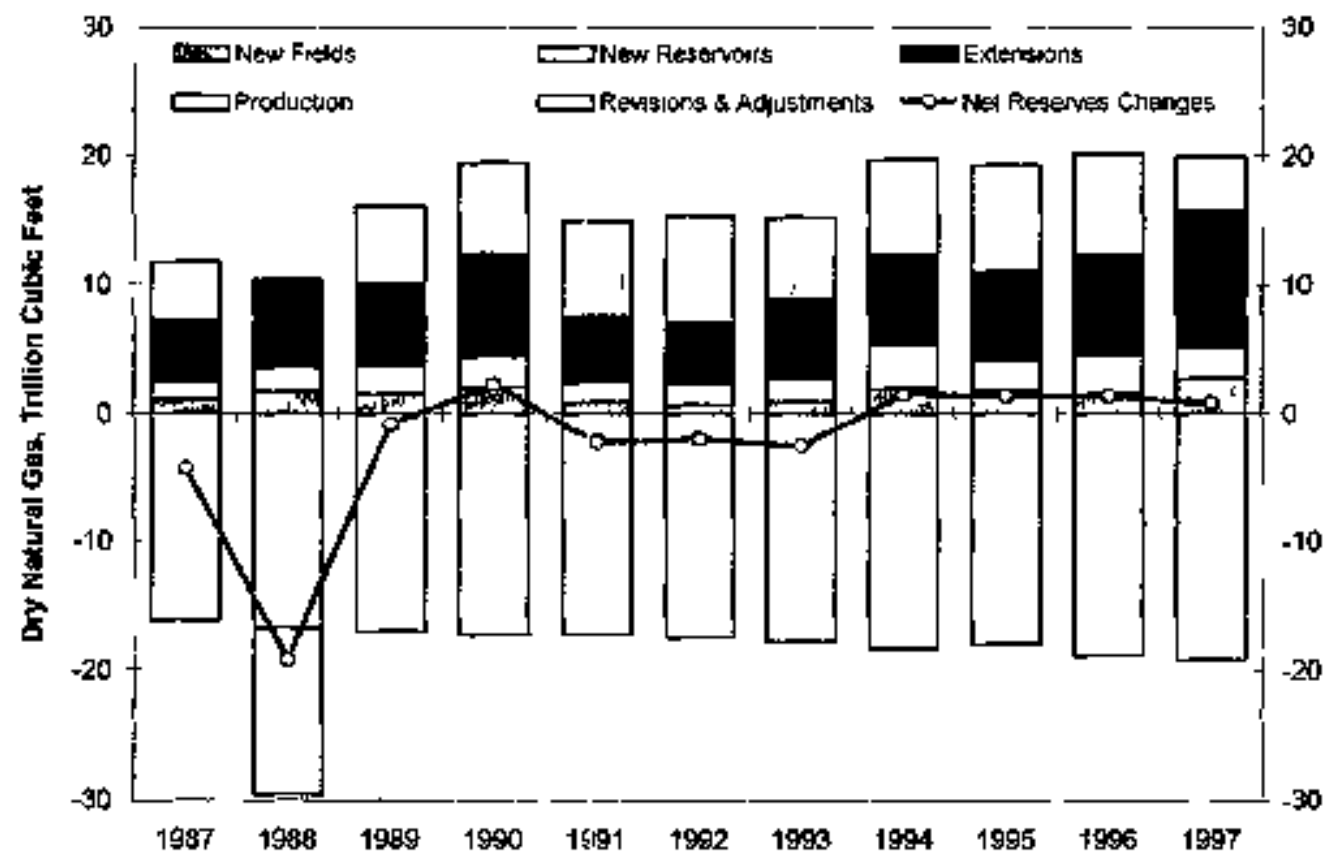

Source U S Crude Ol, Natural Gas, and Natural Gas Lqquids Reserves, 1987-1997 arrulad reports, DOE/EJA-0216 \{10-20\} 
Extensions added 10,585 bilion cubic feet of proved reserves. This is 36 percent more than 1996's extentsions and 68 percent more than the average of extensions in the prior 10 years ( 6,309 billion cubic feet).

New field discoveries added 2,681 billion cubic feet of proved reserves. This is 85 percent more than what was discovered in 1996 and 97 percent higher than the average volume discovered in the prior $10 y$ ears $(1,359$ bülion cubic feet).

New reservoir discoveries in oid fields added 2,382 billion cubic feet of proved reserves. This is 23 percent less than the volume discovered in 1996, but 7 percent higher than the prior 10 -year average $\{2,230$ billion cubic feet).

Revistons ant Adjustntents added 4,312 billion cubic feet of proved reserves. This is 45 percent less than 1996's revisions and adjustments and 85 percent of the prior 10-year averase.

Production removed an estimated 19,211 billion cubic feet of proved reserves from the National total. Gas production increased compared to 1996, for the second consecutive year.

Coalbed methane gas production and reserves are included in the 1997 tolals. However, ElA separately tracks of these reserves in order to record the development and performance of this gas source. Coalbed methane reserves increased in 1997 to a volume of 11,462 billion cubic feet. Coalbed methane accounts for 7 percent of 1997 U.S. dry natural gas reserves and 6 percent of 1997 U.S. dry gas production No Federal tax incentives for new coalbed methane wells have been available for 4 years.

\section{Natural Gas Liquids}

Proved reserves of natural gas liquids increased 150 million barrels to 7,973 million barreis during 1997-2 percent higher than 1996 levels. An increase of 294 million barrels occurred in Alaska, while the lower 48 States ${ }^{x}$ reserves decreased by 144 miltion basrels. Figure 5 shows the natural gas liquids proved reserves levels by major region and Figure 6 shows the components of reserves changes from 1987 through 1997.

Operators seplaced 117 percent of their 1997 natural gas liquids production with reserve additions. Total discoveries accounted for 73 percent of all reserve additions, while rewisions and adjustments accounted for the remaining 27 percent.

Total proved reserves of liquid hydrocarbons (crude oil plus natural gas liquids) were 30,519 million barrels in 1997-an increase of 679 million barrels from the 1996 level. Natural gas liquids represented 26 percent of total liquid hydrocarbon proved reserve in 1997.

\section{Reserves Changes Since 1977}

ElA has callected oil and gas reserves estimates arnually since 1977. Table 2 lists the cumulative totals of the components of reserves changes for crude oil and dry natural gas from 1977 through 1997. Table 2 contains two sections, one for the lower 48 States and another for the U.S. total (which includes Alaska's contribution). Annual averages for each component of reserves changes are also listed, along with the percentage of that particular component's impact on total U.S. proved resetves. In this section, we compare these averages to the 1997 proved reserves estimates as a means of gatrging the past year against history.

Crude Oil: Since 1977 U.S. operators have:

- discovered an average of 813 million barrels per year of new reserves

- revised and adjusted their proved reserves upward by an average of 1,363 million barrels per year from revisions and adjustments

- ended each year with an average net reduction in U.S. proved reserves of 522 milition bartels (the difference between post-1976 average annual production and post-1976 average anuual reserve additions) because production has outpaced reserve additions.

Since 1977, crude oil reserves have been primarily sustained by the extension and development of existing fields (called field growth, reserves growth, or the EIA preferred term: proved ultimate recovery appreciation. See Proved Ultimate Recovery section later in this chapter.), rather than the discovesy of new oil fields. Only 7.6 percent of reserves additions since 1976 were booked as netu field discoveries. Proved ultimate recovery appreciation is the sun of net revisions and adjustments, extensions, and new reservoirs in old fields. Since 1977, the largest component of proved ultimate recovery appreciation for crude oil is upward recisions and adjustments, which accounted for 63 percent of all crude oil reserves additions. The 17,069 million barrels of total discoteries accounted for the remaining 37 percent of reserves additions. 
Figure 5. U.S. Natural Gas Liquids Proved Reserves, 1987-1997

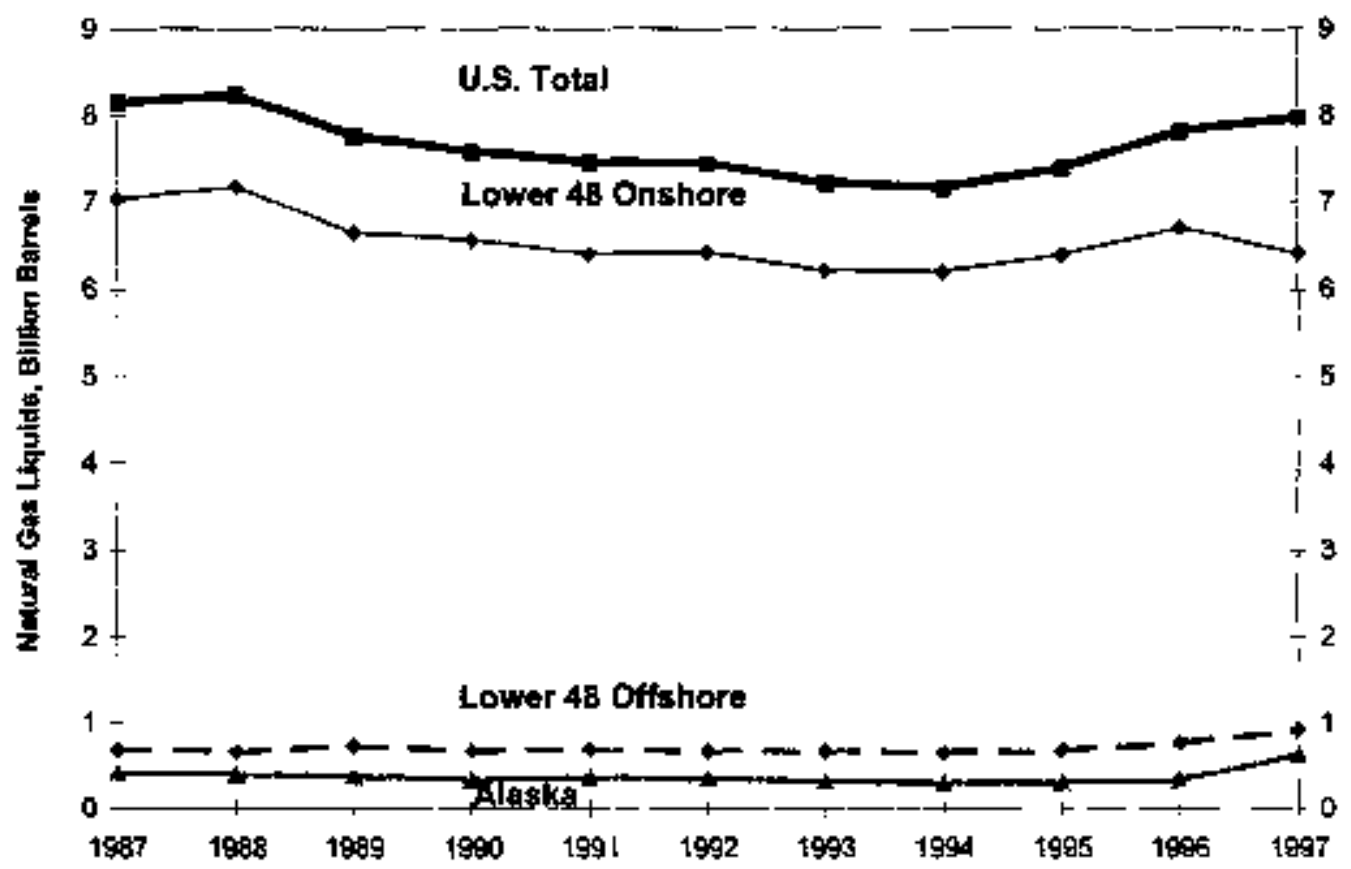

Figure 6. Components of Resterves Changes for Natural Gas Liquids, 1987-1997

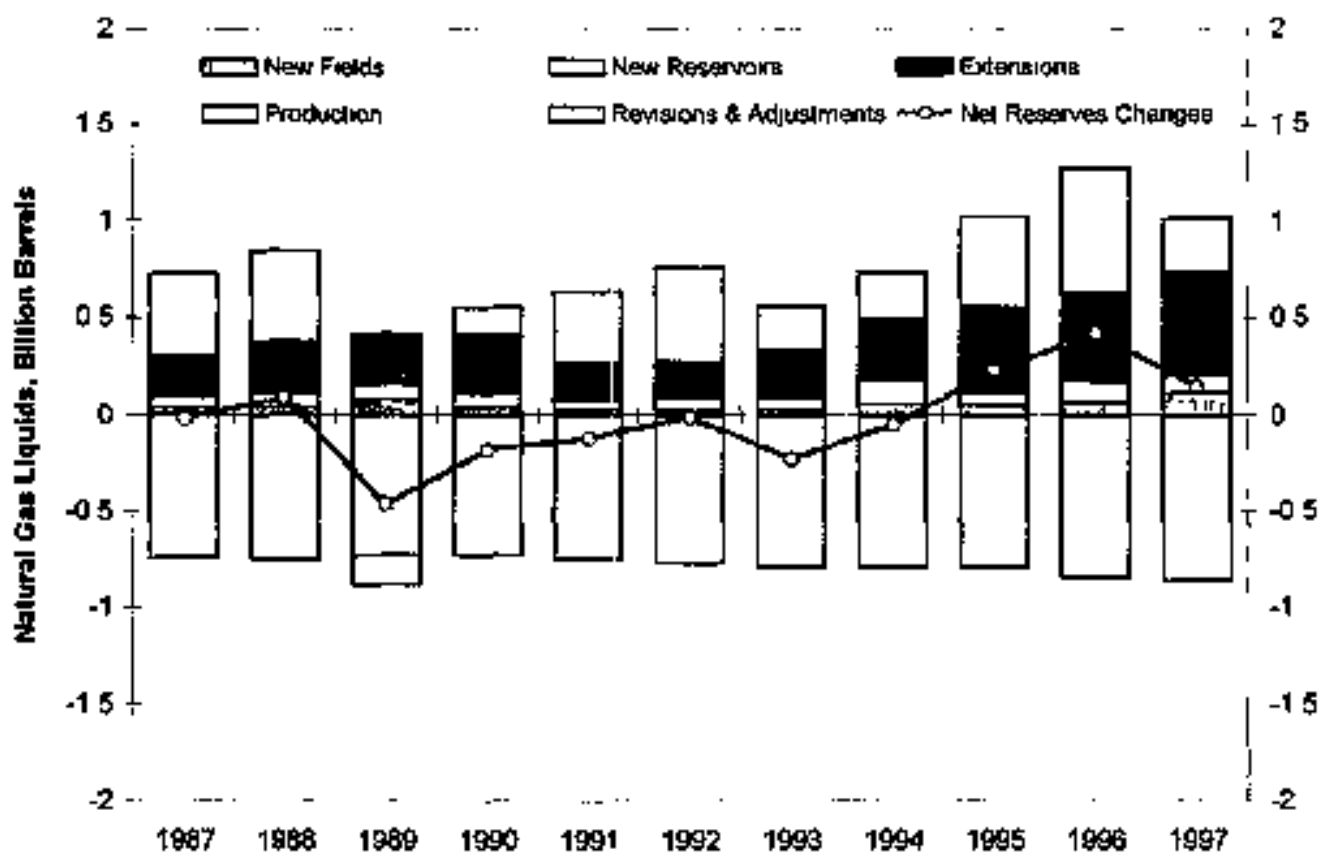

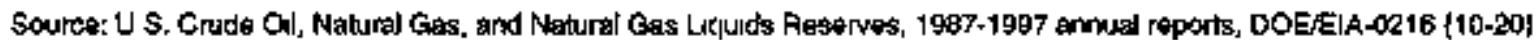




\begin{tabular}{|c|c|c|c|c|c|c|}
\hline \multirow[b]{2}{*}{ Components of Change } & \multicolumn{3}{|c|}{ Lowed AB Stales } & \multicolumn{3}{|c|}{ U.S. Totsl } \\
\hline & Volume & $\begin{array}{c}\text { Average } \\
\text { per } \\
\text { Year }\end{array}$ & $\begin{array}{l}\text { Pertent } \\
\text { of Pepserve } \\
\text { Additions }\end{array}$ & Volume & $\begin{array}{l}\text { Average } \\
\text { per } \\
\text { Yopr }\end{array}$ & $\begin{array}{l}\text { Percent } \\
\text { of Reserva } \\
\text { Addtitions }\end{array}$ \\
\hline & \multicolumn{6}{|c|}{ Crude Oll (milion barrels of 42 U.S. gallons) } \\
\hline Provod Aosorves as of $12 \pi t / 76 \ldots \ldots$ & 24,928 & - & - & 33,502 & - & 一 \\
\hline New Field Discopverles. . . . . . . . . . . & 2.987 & 142 & 8.1 & 3,482 & 186 & 7.6 \\
\hline New Reservoir Discoveries in Odd Fledds. . & 2,879 & 137 & 7.8 & 2,909 & 139 & 6.4 \\
\hline Extensions . . . . . . . . . . & 9,557 & 455 & 26.0 & 10.678 & 508 & 23.4 \\
\hline Total Discowerles . . . . . . . . . . . . . & 15,423 & 734 & 41.9 & 17,069 & 813 & 37.4 \\
\hline Revisions and Actjustments . . . . . . . & 21,348 & 1,017 & 58.1 & $2 B, 620$ & $1, \$ 63$ & 62.6 \\
\hline Totrl Feserve Additions. . . . . . . . & 36,771 & 1,751 & 100.0 & 45,689 & 2,176 & 100,0 \\
\hline Production $\ldots \ldots \ldots \ldots$ & 44,314 & 2,110 & 120.5 & 56,645 & 2,697 & 124.0 \\
\hline \multirow[t]{2}{*}{ Napt Aersorve Change. . . . . . . . } & $\cdot 7,543$ & .358 & -20.5 & $-10,956$ & -522 & -24.0 \\
\hline & \multicolumn{6}{|c|}{ Dry Notural Ges (bition cubic feet at 14.73 psia and $60^{\circ}$ Fahrenheit) } \\
\hline Proved Reservas at of $12 / 51 / 56 \ldots$ & 180,838 & - & - & 213,278 & - & - \\
\hline New Fiekd Discovaries. . . . . . . . . & 41,567 & $\uparrow, 979$ & 12.3 & 41,655 & 1,984 & 12.9 \\
\hline Now Feserwoin Discovelies in Old Fiedis. & 54,139 & 2,578 & 16.0 & 54,503 & 2,595 & 96.8 \\
\hline Extensions . . . . . . . . . . & 156,060 & $7, \mathbf{4 3 1}$ & 46.0 & 156,975 & 7,475 & 48.4 \\
\hline Fotal Diacoveries $\ldots \ldots \ldots, \ldots \ldots$ & 251,765 & 11,989 & $\mathbf{7 4 . 3}$ & 253,133 & 12,654 & $78 . t$ \\
\hline Regvisions and Adjustments ， . . . . . . & 87,274 & 4,750 & 25.7 & 71,009 & 3,391 & 21.9 \\
\hline Total Feserve Addlthons, , . . . . . . & 339,089 & 16,145 & 100.0 & 324,142 & 15,495 & 100.0 \\
\hline Praduction $\ldots+\ldots+\ldots+\ldots \ldots$ & 363,216 & 17,296 & 107.1 & 370,197 & 17,688 & 114,2 \\
\hline Net Apeerve Change. . . . . . . + & $-24,177$ & $-1,151$ & -7.1 & $-46,056$ & $-2,108$ & $-14,2$ \\
\hline
\end{tabular}

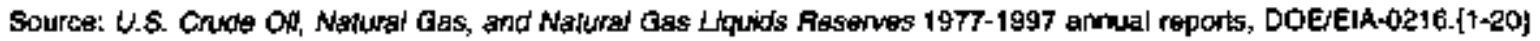

Compared to the average reserves changes since 1977 , 1997 was a good year for crude oil discoveries. 1997's total discoweries of crude oil exceeded the post-1976 U.S. average by 52 percent, while revisionts and adjustments were 5 percent higher than the post-1976 U.S. average.

Only one component of tofal discoveries exceeded the post-1976 averages-new field discoveries. Since 1977 , operators have found only an average of 166 million barrels of crude oil proved reserves per year from new field discoderies. [n 1997, operators discovered 637 million barsels of proved reserves-384 percent of the post-1976 average.

Dry Natural Gas: Since 1977, U.S. operators:

- discovered en average of 12,054 billton cubic feet per year of new reserves

- revised and adjusted their proved reserves upward by an average 3,381 bilition cubjc feet per year

- had an average net reduction in U.S. reserves of 2,193 billion cubic feet per year.
Like crude oil reșerves, natural gas reserves have been sustained primarily by proved ultimate recovery appreciation since 1977. However, extensions rather than net revisions and adjustments is the largest component. Extensions account for 48 percent while reoisions and adjustmenis account for only 22 percent of all teserve additions since 1977 . In recent years, the percentage of net revisions and adjusiments has been as high as 39 percent, but in 1997 it matched the National average of 22 percent.

Compared to the average reserves changes since 197 , 1997 was a good year for natural gas reserve additions from total discoveries. U.S. total dry natural gas reserves increased for the fourth year in a fow. Operators reported 15,648 billion cubic feet of fotal discoveries to their dry natural gas proved reserves-30 percent higher than the post-1976 average (12,054 billion ctbic feet). However, compared to the post-1976 U.S. average, net revisions and adjustments were lower. 


\section{Economics and Drilling}

Economics: Table 3 lists the average annual domestic wellhead prices of crude oil and natural gas, as well as the average number of actve rotary drilling rigs, from 1970 to 1997 .

The U.S. crude oil first purchase price (less the Alaskan North slope) started at an average of $\$ 22.68$ per bajrel in January 1997, but declined to $\$ 17.21$ per barrel in April. Prices wavered until October, when they beçan an unabated five-month decline from $\$ 18.56$ in October 1997 to $\$ 11.53$ in March 1998. In 1997 constant dollars, this price was approximately the same as the price in
1970. The average U.S. crude oil first purchase price (less the Alaskan North Slope) declined from an average $\$ 19.41$ in 1996 to $\$ 17.92$ per barrel in 1997 .

Oil prices vary by region. In Texas the average 1997 price was \$18.64 per barrel, while in California it was $\$ 15.78$ per barrel, and only $\$ 14.84$ per barrel on the Alaskar North Slope. The lowest average crude oil first purchase price in 1997 was for Federal Offshore California oil- $\$ 12.97$ per barrel|21\}.

The average annual wellhead natural gas price increased from $\$ 2.17$ in 1996 to $\$ 2.23$ per thousand cubic feet in 1997. Gas prices started at $\$ 3.42$ per thousand cubic feet in January 1997, but declined to

Table 3. U.S. Average Annual Domostic Wollhead Prices for Crude Oll and Natural Gas, and the Average Number of Active Rotary Drilling Rigs, 1970-1997

\begin{tabular}{|c|c|c|c|c|c|}
\hline \multirow{3}{*}{ Year } & \multicolumn{2}{|c|}{ Crude Oil } & \multicolumn{2}{|c|}{ Natural Gas } & \multirow[b]{3}{*}{ Number of Rigs } \\
\hline & Çurrent & t997 Constant & Current & 1997 Constant & \\
\hline & \multicolumn{2}{|c|}{ (ddollars per barrel) } & \multicolumn{2}{|c|}{ (doflars per thousand eubit feet) } & \\
\hline 1970 & 3.18 & 11.60 & 0.17 & 0.62 & 1,028 \\
\hline 1971 & 3.39 & 11.75 & 0.18 & 0.62 & 976 \\
\hline 1972 & 3.39 & 11.29 & 0.19 & 0.63 & 1,107 \\
\hline 1973 & 3.89 & 12.26 & 0.22 & 0.69 & 1.194 \\
\hline 1974 & 6.87 & 19.91 & 0.30 & 0.87 & 1,472 \\
\hline 1975 & 7.67 & 20.28 & 0.44 & 1.16 & 1,660 \\
\hline 1976 & 8.19 & 20.49 & 0.58 & 1.45 & 1,658 \\
\hline 1977 & 8.57 & 20.18 & 0.79 & 1.86 & 2,001 \\
\hline 1978 & 9.00 & 19.69 & 0.91 & 1.99 & 2,259 \\
\hline 1979 & 12.64 & 25.51 & 1.18 & 2.38 & 2,177 \\
\hline 1980 & 21.59 & 39.89 & 1.59 & 2.94 & 2,909 \\
\hline 1991 & 31.77 & 53.80 & 1.98 & 3.35 & 3,970 \\
\hline 1992 & 28.52 & 45.40 & 2.46 & 3.92 & 3,105 \\
\hline 1983 & 26.19 & 39.98 & 2.59 & 3.95 & 2,232 \\
\hline 1994 & 25.88 & 38.05 & 2.66 & 3.91 & 2,428 \\
\hline 1985 & 24.09 & 34.20 & 2.51 & 3.56 & 1,980 \\
\hline 1986 & 12.51 & 17.32 & 1.94 & 2.69 & $9 \$ 4$ \\
\hline 1987 & 15.40 & 20.68 & 1.67 & 2.24 & 936 \\
\hline 1998 & 12.58 & 16.31 & 1.69 & 2.19 & 936 \\
\hline 1989 & $\$ 5.86$ & 19.73 & 1.69 & 2.10 & 869 \\
\hline 1990 & 20.03 & 23.88 & 1.71 & 2.04 & 7,010 \\
\hline 1991 & 16.54 & 18.97 & 1.64 & 1.68 & 660 \\
\hline 1992 & 15.99 & 17.84 & 1.74 & 1.94 & 721 \\
\hline 1993 & 14.25 & 15.50 & 2.04 & 2.22 & 754 \\
\hline 1994 & 13.19 & 14.01 & 1.85 & 1.96 & 775 \\
\hline 1995 & 14.62 & 15.18 & 1.55 & 1.61 & 723 \\
\hline 1996 & R19.41 & $\$ 9.78$ & $\mathrm{R} 2.17$ & 2.21 & 779 \\
\hline 1997 & 17.92 & 17.92 & 2.23 & 2.23 & 343 \\
\hline
\end{tabular}

R=Revised dala.

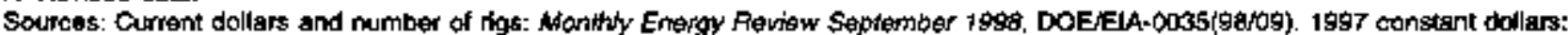

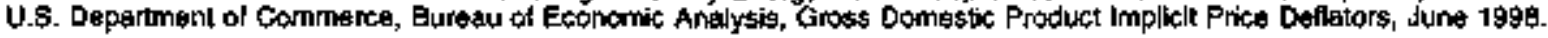


$\$ 1.61$ per thousand cubic feet by March 1997 (the lowest average price of the year). In June, prices tose to $\$ 2.01$, but declined to $\$ 1.91$ in July. In November, prices reached $\$ 277$ per thousard cubic feet, yet declined in December to $\$ 2.17$ per thousand cubic feet, more than a dollar less than prictes of December 1996.(22)

Drilling: From 1996 to 1997, the anmulal average active rig count increased from 779 to 943 (Table 3). The rig count remains well below the peak activity level of 1981 (average 3,970 rigs) but, it must be realized that a comparison of only the bulk number of wells drilled is not representative of drilling's impact on reserves levels over time. Operators are now using significantly improved drilling and seismic exploration technology to dramatically increase their drilling success rate.

Looking first at exploratory wells, there were 3,031 exploratory wells drilled in 1997 (Table 4). Of these, 14 percent were oil wells, 18 percent were gas wells, and 68 percent were dry holes. The total (which includes dry holes) was 4 pereent less than in 1996.

Although the number of active rigs increased in 1997. there were fewer successful exploratory wells. There were 5 percent less exploratory gas wells (Figure 7) and 13 percent fewer exploratory oil wells (Figure 8) than in 1996. On the other hand, as shown in Table 4 , the number of successful development wells increased for both oil and gas.

Figures 9 and 10 show the average volume of discoveries per exploratory well for dry natural gas and oil, respectively, since 1977. The average volume of gas discoveries per exploratory well increased 32 percent in 1997 from the 1996 level. The average volume of oil discoveries per exploratory well in 1997 is 53 percent higher than 1996's level. There were an estimated 27,007 exploratory and development wells drilled in 1997, 19 percent more than in 1996 and slightly higher the average number of wells drilled arnually in the prior 10 years $(26,893)$.

Operators completed more oil and gas wells in 1997 than in 1996. For the fifth year in a row, the number of gas well completions exceeded the number of oil well completions in both the exploratory and development categories.

\section{Reserve-to-Production Ratio and Ultimate Recovery}

\section{R/P Ratios}

The relationship between proved reserves and production levels, expressed as the ratio of reserves to production ( $\mathrm{R} / \mathrm{P}$ ratio) is often used in analyses. For a mature producing area, the $R / P$ ratio tends to be reasonably stable, so that the proved reserves at the end of a year serve as a tough guide to the production level that can be maintained during the following year. Operators report data which yield $R / P$ ratios that vary widely by area depending upon:

- category of operator

- geology and economics

- number and size of new discoveries

- amount of drilling that has occurred.

R/P ratios are an indication of the state of development in an area and, over time, the ratios change. For example, when the Alaskan North Slope oil reserves were booked, the U.S. R/P ratio for crude oil increased because significant production from these reserves did not begin unbil 7 years after booking due to the need to first build the Trans Alaska pipeline. The U.S. R/P ratio for crude oil decreased from 11,1-to-1 to 9.4-to-1 between 19/7 and 1982, as Alaskan North Slope oil production reached high levels.

In 1997, U.S. crude oil proved reserves increased and oil production decreased - resulting in a slight increase in the National average $R / P$ ratio, from 10.1 to 10.6 .

Figure 11 shows the U.S. R/P ratio trend for crude oil since 1945. After World War II, increased drilling and discoveries led to a greater $R / P$ ratio. Later, when drilling found fewer reserves than were produced, the ratio became smaller. $R / P$ Ratios also vary geographically. Less developed areas of the country, such as the Pacitic offshore, have higher $R / P$ ratios for crude oil than the 1997 National avetage of 10.6-to-1. Other areas with relatively high $R / P$ ratios are the Permian Basin of Texas and New Mexico, and California, where enhanced oil recovery techniques such as carbon dioxide $\left(\mathrm{CO}_{2}\right)$ injection or 


\begin{tabular}{|c|c|c|c|c|c|c|c|c|}
\hline \multirow[b]{2}{*}{ Yoser } & \multicolumn{4}{|c|}{ Explotatory } & \multicolumn{4}{|c|}{ Total Exploralory and Development" } \\
\hline & 에 & Gas & Dry & Total & Oil & Cas & Dry & Total \\
\hline 1970 & 763 & 478 & 6,193 & 7,434 & 13,043 & 4,031 & 11,099 & 28,173 \\
\hline 1971 & 664 & 472 & 5,995 & 7,131 & 11.903 & 3,983 & 10,382 & 26,268 \\
\hline 1972 & 690 & 659 & 6,202 & $7, \mathbf{5 5 1}$ & 11,437 & 5.484 & 11,013 & 27,934 \\
\hline 1973 & 642 & 1,067 & 5,952 & 7,661 & 10,167 & 6,983 & 10,320 & 27,420 \\
\hline 1974 & 859 & 1,190 & 6,833 & 8,862 & 13,647 & 7.138 & 12,116 & $32,90 t$ \\
\hline 1975 & 982 & 1,248 & 7,129 & 9,359 & 16,948 & 8,127 & 13,646 & 38,721 \\
\hline 1976 & 1,086 & 1.346 & 6,772 & 9,204 & 17,688 & 9,409 & 13,758 & 40,855 \\
\hline 1977 & $\uparrow, 164$ & 1,548 & 7,283 & 9,995 & 18,745 & 12,122 & 14,985 & 45,852 \\
\hline 1978 & 1,171 & $1,77 t$ & 7,965 & 10,907 & 19,181 & 14,413 & 16,551 & 50,145 \\
\hline 1979 & 1,321 & 1,907 & 7,437 & 10,665 & 20,851 & $\{5,254$ & 16,099 & 52,204 \\
\hline 1980 & 1,764 & 2,081 & 9,039 & 12,884 & 32,639 & 17,333 & 20,638 & 70,610 \\
\hline 1981 & 2,696 & 2,514 & 12,349 & 17,499 & 43,598 & 20,166 & 27,789 & 91,553 \\
\hline 1982 & 2,431 & 2,125 & 11,247 & 15,803 & 39,199 & 16,979 & 26,219 & 84,397 \\
\hline 1983 & 2,023 & 1,593 & 10,148 & 13,764 & 37,120 & 14,564 & 24,153 & 75,837 \\
\hline 1984 & 2,197 & 1,521 & 11.278 & 14,996 & 42,605 & 17,127 & 25,681 & 85,413 \\
\hline t985 & 1,679 & 1,189 & 8,924 & 14,792 & 35,118 & 14,168 & 21,056 & 70,342 \\
\hline 1906 & 1,084 & 793 & 5,549 & 7,426 & 19,097 & 8,512 & 12,661 & 40,270 \\
\hline 1987 & 925 & 753 & 5,049 & 6,727 & 16,164 & 8,055 & 11,101 & $\$ 5,320$ \\
\hline 1988 & 855 & 730 & 4,691 & 6.276 & 13,636 & 8,555 & 10,041 & 32,232 \\
\hline 1989 & 607 & 707 & 3,924 & 5,238 & 10,204 & 9,539 & $8,18 \theta$ & 27,931 \\
\hline 1990 & 653 & 691 & 3.715 & 5,059 & 12,198 & 11,044 & 8,309 & 31,551 \\
\hline 1991 & 593 & 538 & 3,312 & 4,443 & 11.770 & 9,526 & 7,599 & 28,895 \\
\hline 1992 & 496 & 424 & 2,510 & 3,430 & 8,757 & $B, 209$ & 6,118 & 23,094 \\
\hline 1993 & 500 & 544 & 2,470 & 3,514 & 8,365 & 10,005 & 6,295 & 24,665 \\
\hline 1994 & 566 & 720 & 2,400 & 3,686 & 6,690 & 9,538 & 5,279 & 21,507 \\
\hline 1995 & 541 & 567 & $2,+98$ & 3,306 & 7,627 & 8,337 & 5,075 & 21,039 \\
\hline 1996 & 481 & 560 & 2,130 & 3,171 & 8.301 & 9,185 & 5,220 & 20,706 \\
\hline 1997 & 418 & 534 & 2,079 & 3,031 & 10,268 & 11,245 & 5,494 & 27,007 \\
\hline
\end{tabular}

axcludes service wells and stralichrephlc and core tesilng.

ball diilling counts for the years 1973 -1996 have been revisht.

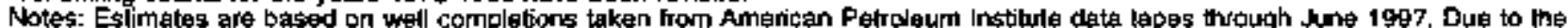
mettord of estimation, data shown are frequeritly revised. Data s re no longer rounded to netarest 10 wells.

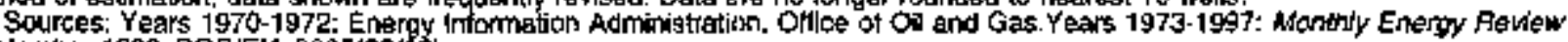

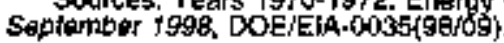

steamtlooding have improved recoverability of oil in old, mature fields. Areas that have the lowest $\mathbf{R}_{i} \mathbf{P}$ ratios, like the Mid-Continent region, usually have many older fieids. There, new technologies such as horizontal drilling have helped add reserves equivalent to the annual production, keeping the regional reserves and $\mathrm{R} / \mathrm{P}$ ratio for oil relatively stable.

Figure 12 shows the historical $R /$ P ratio for wet natural gas since 1945. Prior to $1945, R / P$ ratios were very high since the interstate pipeline infrastructure was not well developed. The market for and production of natural gas grew rapidly after World Wat II, lowering the R/P ratio. The U.S. average $R / P$ ratio for natural gas decreased in 1997, as the proportional increase in reserves was not as much as the increase in production.

Different marketing, transportation, and production characteristics for gas are seen when looking at regional average $R / P$ ratios, compared to the 1997 U.S. average $R / P$ ratio of about $8.7-10-1$. The areas with the higher range of $R / P$ ratios are the less developed or less productive areas of the country, such as the Pacific offshore and the Rockies, and also include areas such as 
Figure 7. U.S. Exploratory Cas Well Completions, 1977-1997

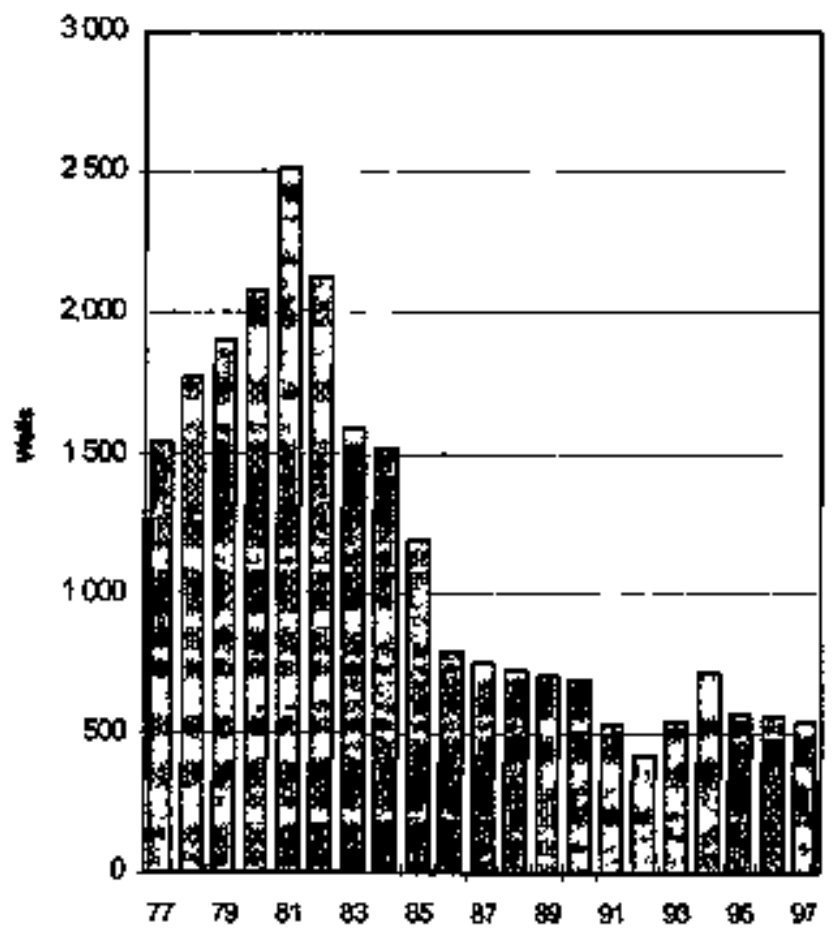

Figure 8. U.S. Exploratory Oil Well Completions, $1977-1997$

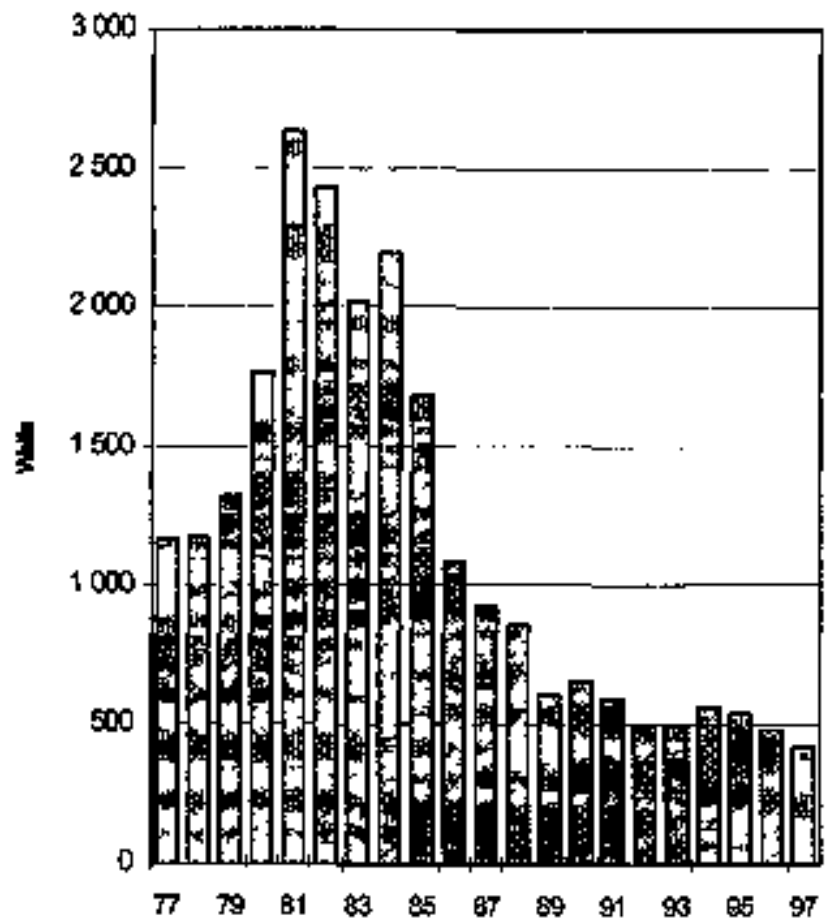

Figure 9. U.S. Total Discoveries of Dry Naturel Gas per Exploratory Gas Well Completion, 1977-1997

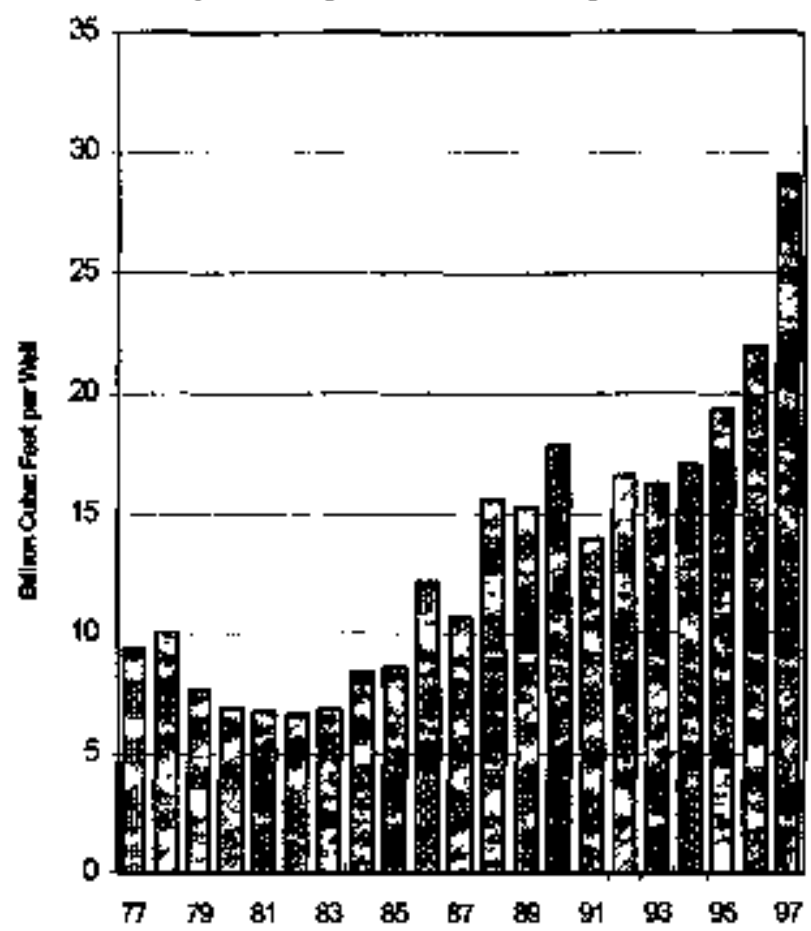

Figure 10. U.S. Total Discoveries of Crude Oil per Explaratory Oil Well Completion, 1977-1997

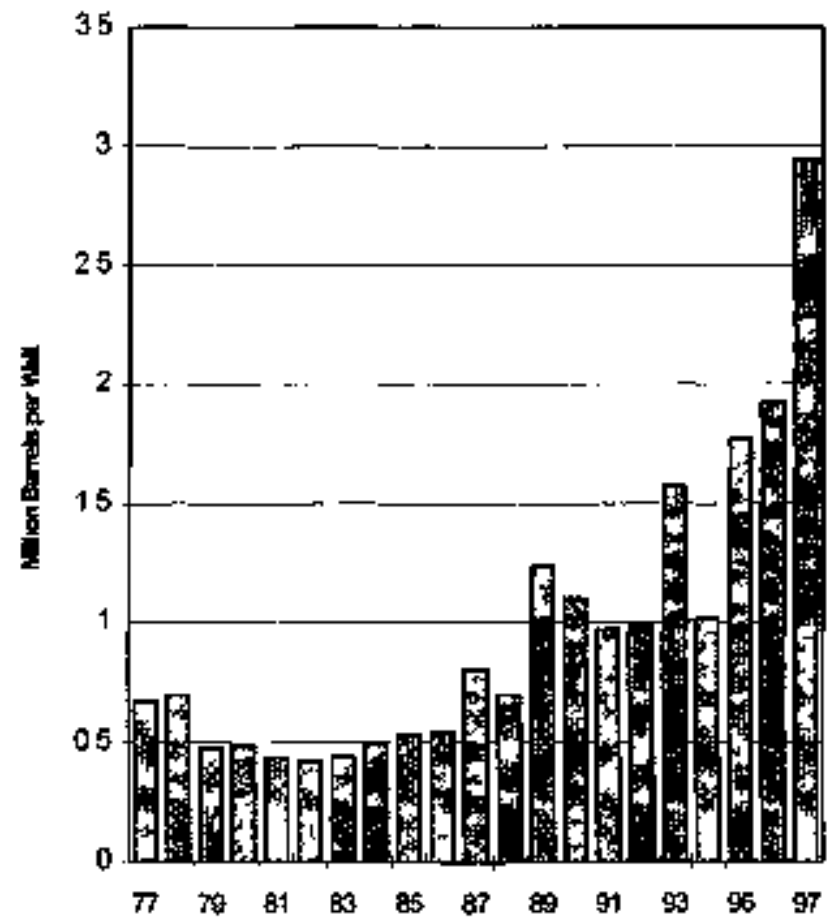

Source Energy Information Admunlstraten, Olfice of Oll and Gas 
Alabama and Colorado where considerable booking of coalbed methane reserves has recently occurred. Several major gas producing areas have $R / P$ ratios below the National average, particularly Texas, the Gulf of Mexico Federal Offshore, and Oklahoma. The R/P ratio of these three areas combined decreased from 7.0-to-1 in 1996 to 6.8-to-1 in 1997, and is below the National 1997 average.

\section{Proved Ultimate Recovery}

EIA in past reports has defined Ultimate Recovery as the sum of proved reserves and cumulative production. However, despite EIA's clear definition, the volume presented by ElA has often been mistrsed or misinterpreted as the maximum recoverable volune of resources for an area. This neglects the addition of proved reserves over time through ultimate recovery appreciation (a.k.a reserves growth or field growt'h) and has led some to make overly-pessimistic resource assessments for the United States. ElA is therefore introducing a new term, Proved Llitinate Recovery:

Proved Ultimate Recovery is the sum of proved reserves and cumulative production. It is expected to change over time for any field, group of ficlds, State, or Country. Proved Ultimate Recovery does not represent the maximum recoverable volume of resources for an area. It is instead a gauge of how much has already been produced plus proved reserves. Proved reserves of crude oil or natural gas are the estimated quantities of petroleum which geological and engineering data demonstrate with reasonable certainty to be recoverable in future years trom known reservoirs under existing economic and operating conditions. When dekerministic proved reserves estimation methods are used, the term reasonable certainty is intended to express a high degree of confidence that the estimated quantities will be recovered. When probabilistic methods are used there should be at least a 90 percent probability that the actual quantities recovered will exceed the estimate.

Figures 13 and 14 show successive estimates of proved ultimate recovery and its components, proved reservess and cumulative production, for crude oil plus leases condensate, and wet natural gas, from 1977 through 1997. They illustrate the continued appreciation (growth) of proved ultimate recovery over time.

In 1977 , U.S. crude oil and lease condensate proved reserves were 33,615 million barrels. Cumulative praduction for 1977 through 1997 was 57,258 million barrels. This substantially exceeds the 1977 proved reserves, but at the end of 1997 there were still 23,887 million barrels of crude oil and lease condensate proved reserves. Therefore, the Nation's estimated proved ultimate recovery of crude oil was fundamentally increased during this period owing to the proved ultimate recovery appreciation process (continued development of old fields). In tact, only 7.6 percent of proved reserves additions of crude oil were booked as new field discoveries from 1976 through 1997. The rest was from proved reserves categories included in the proved ultimate recovery apprecjation process (new reserooir discoweries in old felds, extensions, and revisions and adjustments.) A stgnificant part of the total proved ultimate recovery appreciation came from the proved ultimate recovery appreciation of those new fields discovered between 1976 and 1997.

Similarly, the 1977 wet natural gas proved reserves were 209,490 billion cubic feet, and cumulative wet gas production from 1977 through 1997 was 371,368 billion cubic feet. Cumulative wet gas production exceeded the 1977 reserves by 161,878 billion cubic feet, but at the end of 1997 there were still 175,721 billion cubic feet of wet natural gas proved reserves, for the same reasons. Only 12.9 percent of proved reserve additions of natural gas were booked as new field discoveries from 1976 through 1997.

\section{International Perspective}

\section{International Reserves}

The EIA estimates domestic oil and gas reserves but does not systematically estinate worldwide reserves. As shown in Table 5, intemational reserves estimates are presented in two widely eirculated trade publications. The world's total reserves are estimated to be roughly 1 trillion barrels of oil and 5 quadrillion cubic feet of gas.

The United States ranked 11th in the world for proved reserves of crude oil and 6th for natural gas in 1997. unchanged from 1996. A comparison of EIA's U.S. proved reserves estimates with worldwide estimates obtained from other sources shows that the United States had about 2 percent of the world's total crude oil proved reserves and over 3 percent of the world's total matural gas proved reserves at the end of 1997. There are sometimes substantial differences between the estimates from these sources. The Oil \& Gas Journal reported oil reserves for the United Arab Emirates at 
Figure 11. Reserves-to-Production Ratios for Crude Oil, 1945-1997

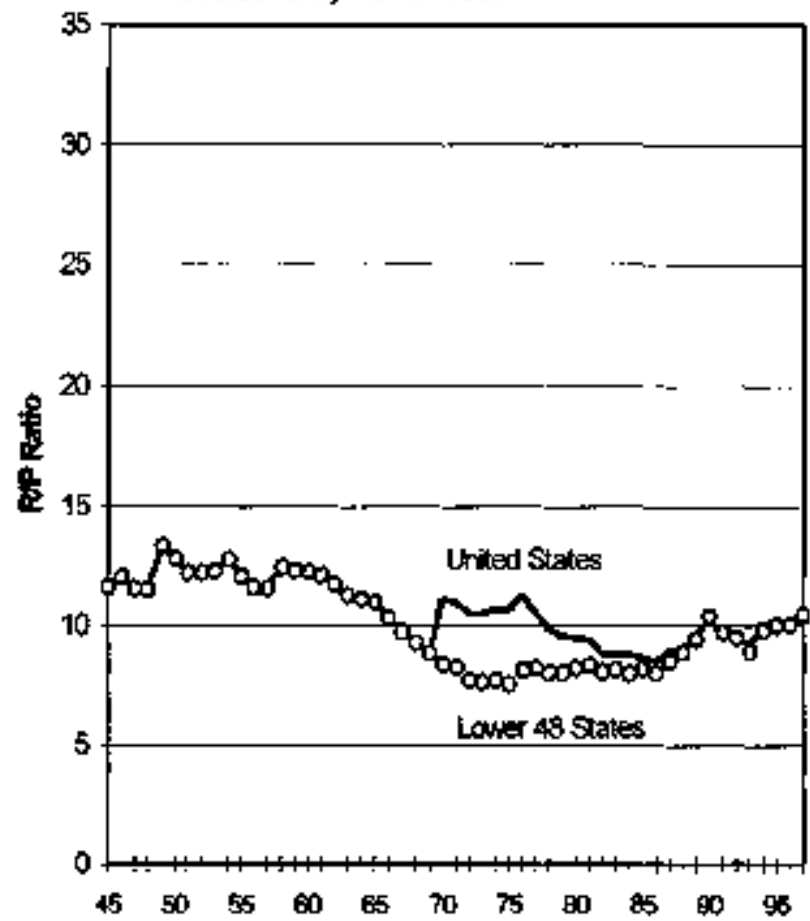

Figure 12. Feserves-to-Production Fatlos for Wot Natural Gas, 1945-1997

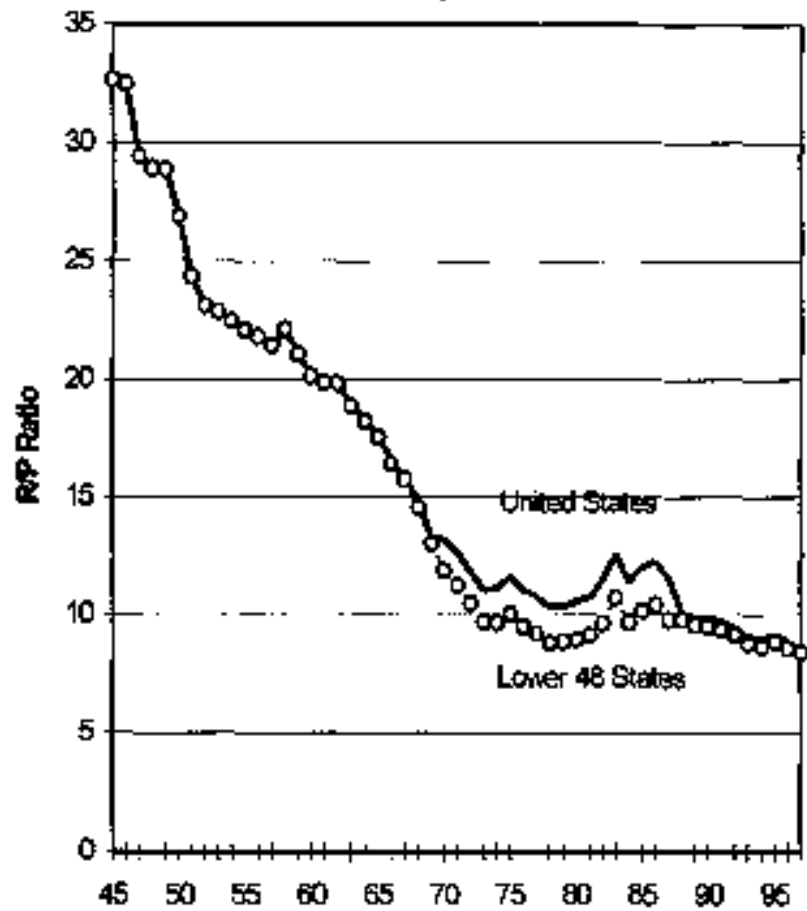

Figure 13. Components of Proved Ultimate Recovery for Crude Oil and Leape Condensate, 1977-1997

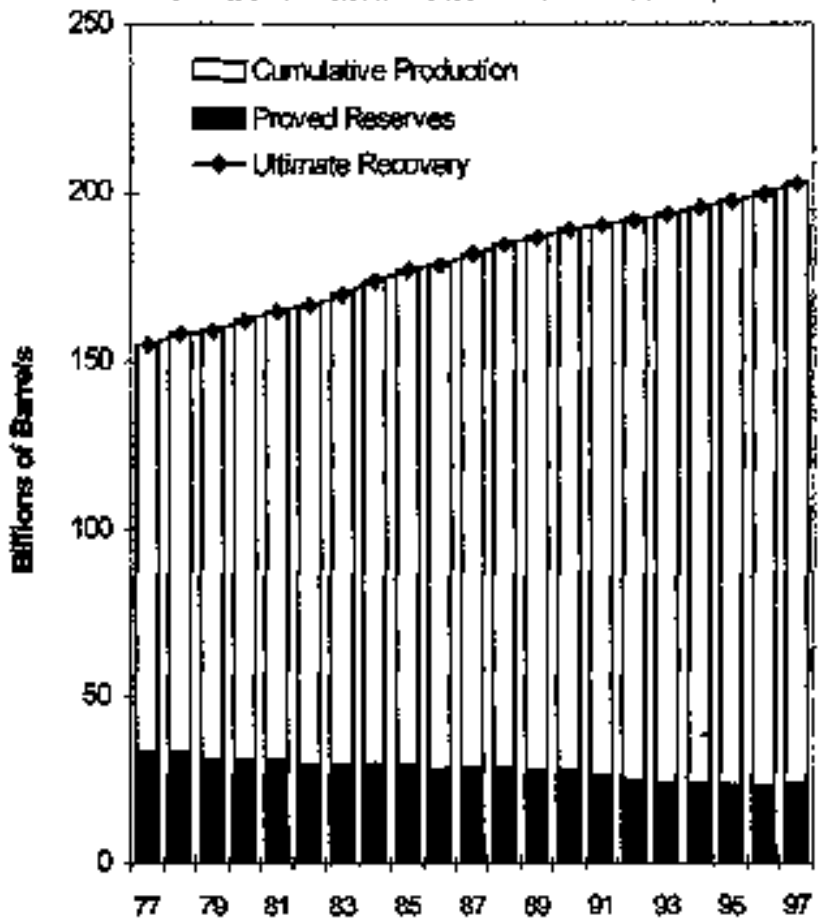

Figure 14. Components of Proved Uhtimate Recovery for Wet Natural Gas, 1977-1997

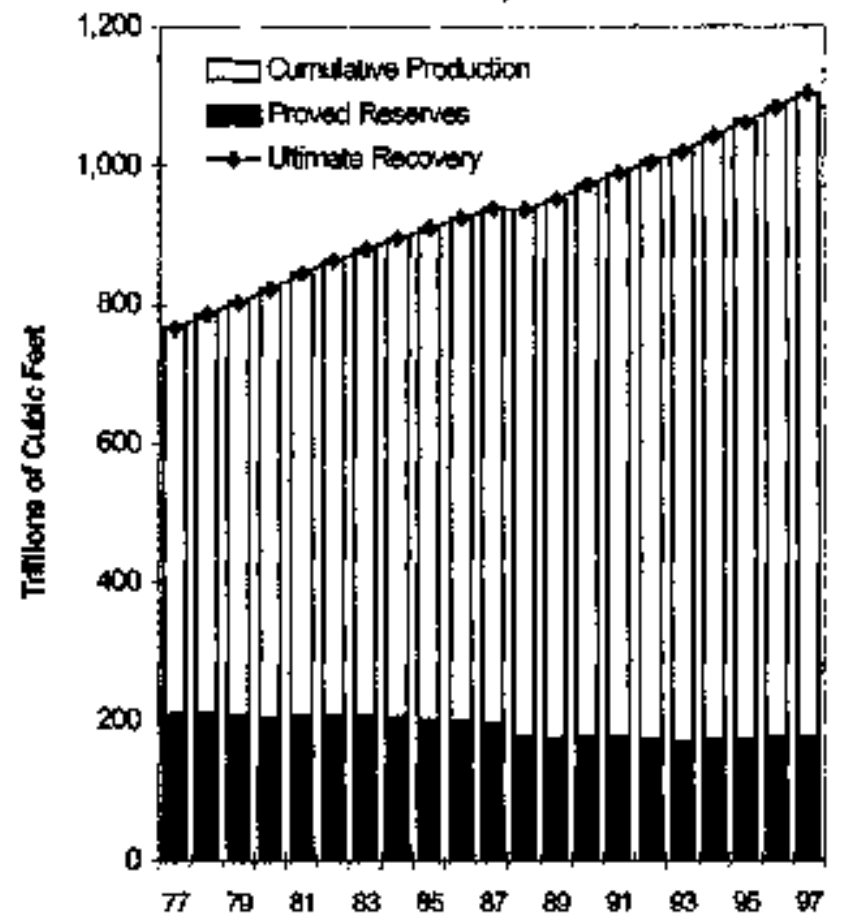

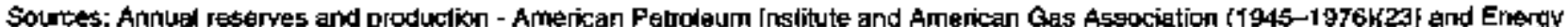
Information Administratlon, Office of Oil and Gas $(1977-1997)[1.20)$. Cumulative produclion; US. Oll and Gas Reserves by Year of Fiofd Discovary $\{1977-1988\} .\{24\}$ 
Table 5. Intomational Oil and Natural Gas Feserves as of December 31, 1997

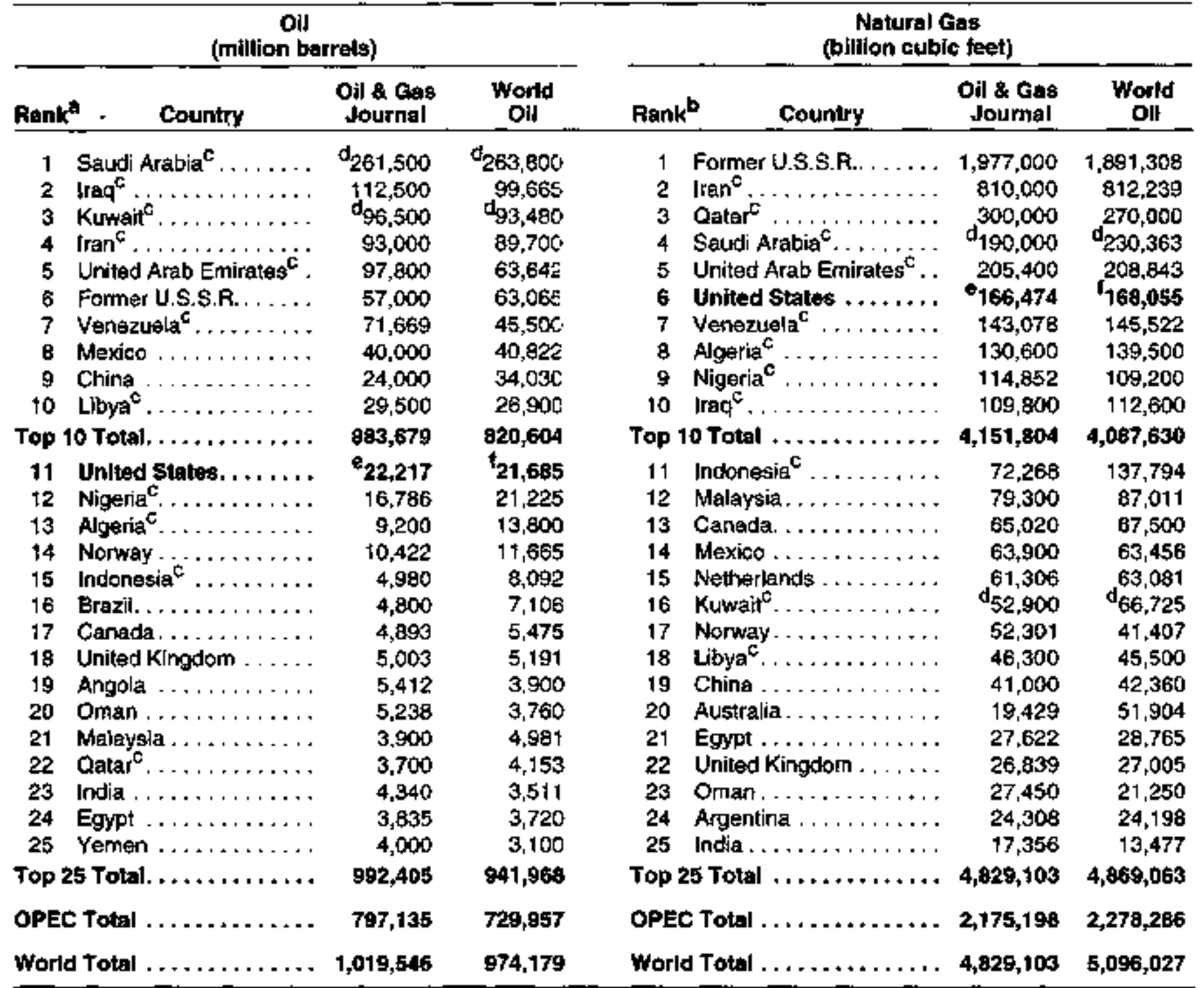

\footnotetext{
apank is based on an average of oll reserves reportod by Oit \& Ges Joennel and Wortd Oil.

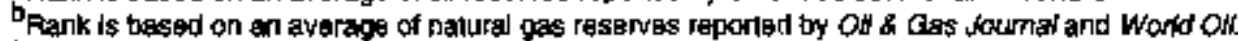

cMember of the Drganization of Petroleum Exporting Counlrles (OPEC).

drichudes one-hali of the reserves in the Neutral Zone.

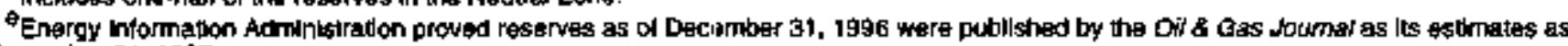
of December 31, 1997.

Worfd Oil estimate.

Note: The Energy Inlormation Administration does not centify tu eșe Inlemational reaserves date, but reproduces the inlormation as a mallęr of convenience for the reader.

Sources: PennWen Publishing Company, ON and Gas downal, December 29, 1997, pps. 30-39. Bull Fublishing Company, Worrd ON, Augusi, 1998, p. 29.
} 
about 98 billion barrels. This is about 50 percent higher than the World Oil estirnate of 64 billion. One reason (arnong mary) for these differences is that condersate is often included in foreign oil reserve estimates.

The Ot \& Gas founal $\{25\}$ estimate for world oil reserves remained about the same in 1997, while the World Oil\{26\} estimate decreased 16 percent, mostly due to World Oil's reevaluation of Venezuela and the former Soviek Unjon's proved reserves.[27] For world gas reserves, the $O$ il $\&$ cas Joumal reported a 3 percent increase, while World Oil reported a 2 percent decrease.

Several foreign countries have oil reserves considerably larger than those of the United States. Saudi Arabian oil reserves are the largest in the world, dwarfing U.S. oil reserves. Iraqi oil reserves are almost 5 times U.S. reserves. Closer to home, Venezuela has more than double and Mexico has around 84 percent more than the United States' oil reserves. (Based on averages of the World $\mathrm{Oil}$ and Oil \& Gas Journal estimates).

\section{Petroleum Consumption}

The United States is the world's largest energy consumer. The ElA estimates energy consumption and publishes it in its Annual Energy Review. $\{28\}$ In 1997 .

- The U.S. consumed $94,209,000,000,000,000 \mathrm{Btr}$ of energy (94.21 quadrillion Btu).

- 63 percent of U.S. energy consumption was provided by petroleum and natural gas-crude oil and nafural gas liquids combined (39 percent), and raturral gas (24 percent).

- US. petroleum consumption was about 18.6 million barrels of oil and natural gas liquids and 60.3 billion cubjic feet of dry gas per day.

\section{Dependence on Imports}

The United States remains heavily dependent on imported oil and gas to sabisfy its ever-increasing appetite for energy. In 1996, the U.S. was dependent on net petroleum imports for 46 percent of energy consumption. in 1997, it increased to a 20-year high of 53 percent.

Net gas inports increased slightly in 1997 to 2.8 trillion cubic feet, which is approximately 13 percent of consumption. Almost all of this gas was pipelined from Canada, some came from Mexico, though Mexico remains a net inporter of natural gas from the U.S., and a very snatl amount of liquefied natural gas was imported from Algeria and Australia.
Venezuela, Saudi Arabia, Canada, Mexico, and Nigeria were the primary foreign suppliers of petroleum to the United States. Price-tompetitive Canadian gas exports continue to capture an increasing share of the U.S. market. [29]

\section{List Of Appendices}

Appendix A: Reserves by Operator Production Size Class - How much of the National total of proved reserves are owned and operated by the large oil and gas corporations? Appendix A separates the large operators from the small and presents reserves data according to operator production size classes.

Appendix B: Top 100 Oil and Gas Fields - What fields have the most reserves and production in the United States? The top 100 fields for oil and natural gas out of the inventory of more than 45,000 oil and gas fields are listed in Appendix B. These fields hold two-thirds of U.S. crude oil proved reserves.

Appendix C: Conversion to the Metric System - To simplify international comparisons, a summary of U.S. Crude Oil, Natural Gas, and Natural Gas Liquids Reserves expressed in metric units is included as Apperdix C

Appendix D: Historical Resterves Statistics Appendix D contains selected historical reserves data presented at the State and National level. Readers interested in a historical look at one specific State or region can review these tables. We have again íncluded Table D9, Deepwater Production and Proved Reserves of the Culf of Mexico Federal Offshore 1992-1997, due to expressed interest from the industry regarding this area. Table D9 contains the production and proved reserves for 1992-1997 for the Gulf of Mexico Federal Offshore region by waker depths greater than 200 meters, and less than 200 meters.

Appendix E: Summary of Data Collection Operations - This report is based on two EIA surveys. Proved reserves data is collected arunually from U.S. oil and gas field operators on Form EIA-23. Natural gas liquids production data is collected annually from U.S. natural gas plant operators on Form EIA-64A. Appendix $E$ describes survey designs, response statistics, reporting requirements, and sampling frame maintainance.

Appendix F: Statistical Considerations - The EIA strives to maintzin or improve the accuracy of its reports. Sínce complete coverage of all oil and gas 
operators is impractical, the ElA has adopted sound statistical methods to impute data for those operato s not sampled and for those data elements that smaller operators are not required to file. These methods are described in Appendix $F$.

Appendix G: Estimation of Reserves and Resources Reserves are not measured directly. Reserves are estimated on the basis of the best geological, engineering, and economic data available to the estimator. Appendix $G$ describes reserve estimation techniques commonly used by oil and gas field operators and ElA personnel when in the field performing quality assurance checks. A discussion of the relationship of reserves to overaill U.S. oil and gas resources is also included.

Appendix H: Maps of Selexted State Subdivisions Certain large producing States have been subdivided into smaller regions to allow more specific reporting of reserves data. Maps of these States identifying the smaller regions are provided in Appendix $\mathrm{H}$.

Appendix I: Annual Survey Forms of Damestic Oil and Gas Reserves - Samples of Form ElA-23 and Form ElA-64A are presented in Appendix 1.

Glossary - Contains definitions of many of the techrical tems used in this report. 


\section{Crude Oil Statistics}

The United States had 22,546 miltion barrels of crude oil proved reserves as of December 31, 1997. This is 2 percent ( 529 million barrels) more than in 1996 and is the first time in 10 years that crude oil proved reserves have increased. Reserues addifions of crude oil replaced 125 percent of 1997 oil production (Figure 15).

Figure 15. Reserve Additlons Replace 125 Percent of U.S. Oil Production in 1997

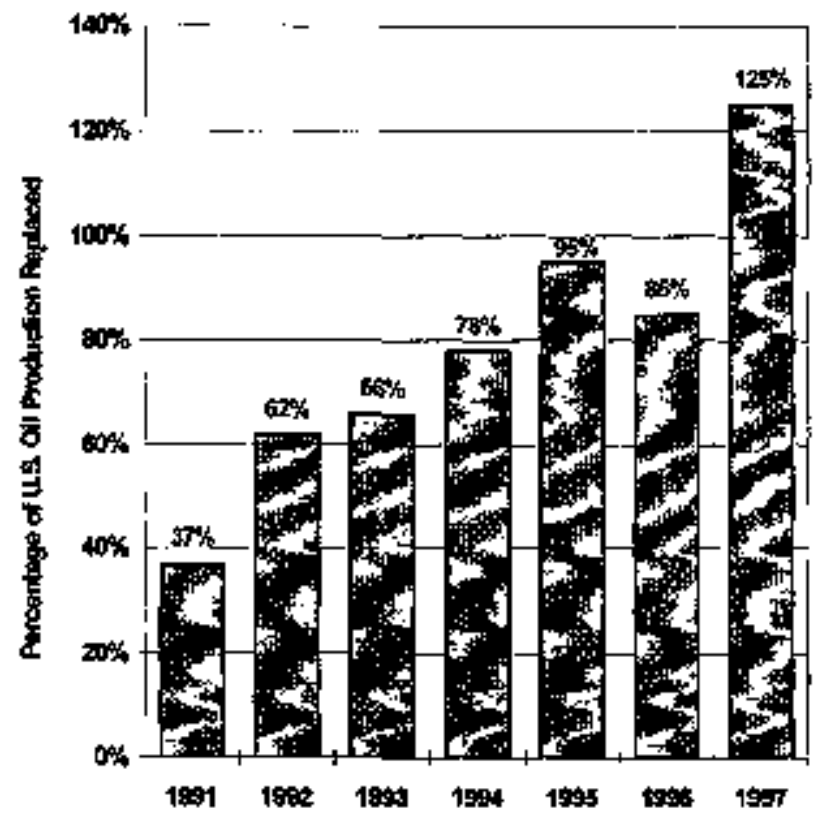

Soulte: Energy Intomution Adrmunstrathon, Office of OAl and Gas.

Continued exploration and development in the Gulf of Mexico Federal Offshore (especially in deep water), fjeld extensions in Texas, new field discoveries in Alaska, and large upward revisions in California's heavy oil fields all combined in 1997 to create the first increase in crude oil proved reserves in a decade. Lower-48 States crude oil proved reserves increased almost 4 percent (642 million barrels) in 1997.

Over the past decade, U.S. crude oil proved reserves had been declining (Figure 1) an average of 2 percent per year. The 1997 increase interrupts this trend, although it remains to be seen if the application of new technology can sustain this level of reserves additions. It is expected that the declining trend will reestablish itself in the future.

\section{Proved Reserves}

Table 6 presents the U.S. proved neserves of crude oil as of December 31, 1997, by selected States and State subdivisions.

Figure 16 maps 1997 crude oil proved reserves by area. The following four areas account for 78 percent of US. crude oil proved reserves:

\begin{tabular}{lc} 
Area & $\begin{array}{c}\text { Percenl of } \\
\text { U.S. Oil Peserves }\end{array}$ \\
\hline Texas & 25 \\
Alaska & 23 \\
Califomia & 17 \\
Gulf of Mexico Federal Otishore & 13 \\
Area Totad & 78
\end{tabular}

Of these four areas, California and the Gulf of Mexico Federal Offshore increased their reserves in 1997, while Alaska and Texas had small decreases in crude oil proved reserves.

\section{Dlscussion of Reserves Changes}

Figure 77 maps the change in crude oil proved reserves from 1996 to 1997 by area. Here's how the top four areas fared conpared to the total United States:

\begin{tabular}{lc} 
Area & $\begin{array}{c}\text { Change in } \\
\text { U.S. Oll Reservos } \\
\text { (milion bamels) }\end{array}$ \\
\hline Texes & -49 \\
Alaska & -113 \\
Calfornia & +313 \\
Gulf of Mexico Federal Oftshom & +382 \\
Area Total & +539 \\
U.S. Toted & +529
\end{tabular}

Figure 2 in Chapter 2 shows the components of the changes in crude oil proved reserves for 1997 and the preceding 10 years.

\section{Total Discoveries}

Total discoteries are those new reserves attributable to extensions of existing fields, new field discoveries, and new reseruoir discoveries in old fields. They result from the drilling of exploratory wells. 
Table 6. Crude Oil Proved Reservee, Reserves Cluanges, and Production, 1997 (Millon Barrels of 42 U.S. Gallons)

\begin{tabular}{|c|c|c|c|c|c|c|c|c|c|}
\hline \multirow[b]{2}{*}{ Stote and Subdhtogn } & \multirow[b]{2}{*}{$\begin{array}{l}\text { Pybinghed } \\
\text { Prowed } \\
\text { Ploperves } \\
1251 \text { ist }\end{array}$} & \multicolumn{7}{|c|}{ Changes In Fooserves During 1997} & \multirow[b]{2}{*}{$\begin{array}{l}\text { Proved } \\
\text { Proterves } \\
\text { 12t31iter }\end{array}$} \\
\hline & & $\begin{array}{l}\text { Adjugtmenta } \\
\text { [+, }\end{array}$ & $\begin{array}{c}\text { Pimvipan } \\
\text { mertatiol } \\
1+1\end{array}$ & $\begin{array}{c}\text { Powndon } \\
\text { Deorotents } \\
\text { (-) }\end{array}$ & $\begin{array}{c}\text { Extentions } \\
\text { (+) }\end{array}$ & 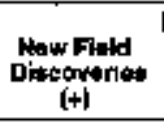 & 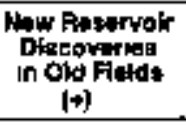 & $\begin{array}{c}\text { Eglimated } \\
\text { Production } \\
(-1) \\
\end{array}$ & \\
\hline Alaskin & 5,274 & 6 & 274 & 55 & 18 & 117 & 0 & 473 & 5,161 \\
\hline 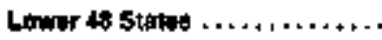 & . 16,743 & 514 & 1,724 & 1,020 & 459 & 620 & 118 & 1,656 & 17,386 \\
\hline Alabama & 45 & 0 & 16 & 4 & 0 & 0 & 0 & 10 & 47 \\
\hline Atkerisst & 58 & 8 & 5 & 4 & 0 & 0 & 0 & 6 & 45 \\
\hline Calloma & $3,43 ?$ & 277 & 324 & 49 & 38 & 0 & 8 & 286 & 3,750 \\
\hline Coagdel Regon Dnthorit & 125 & 21 & 5 & 15 & 10 & 0 & 1 & 23 & 430 \\
\hline Los Angeles Basin Onshore & 234 & 45 & 24 & 15 & $D$ & 0 & 0 & 20 & abB \\
\hline Sen Jaaquin Easan Conshore & 2597 & 209 & $28 \mathrm{e}$ & 19 & 22 & 0 & 7 & 221 & 2.871 \\
\hline Strte Olfisxore & 181 & a & 13 & 0 & 0 & 0 & 0 & $2 t$ & $18 t$ \\
\hline Colomede & 231 & 13 & 9 & 37 & 3 & 0 & 0 & 21 & 199 \\
\hline Flonds & 97 & -1 & 5 & 4 & 0 & 0 & 0 & 6 & 91 \\
\hline Whrowe & 94 & -9 & 24 & 6 & 0 & 0 & 0 & 11 & 92 \\
\hline Indegna & 11 & 3 & 1 & 3 & 0 & 0 & 0 & 2 & $b_{10}$ \\
\hline Kansas & 286 & 13 & 51 & $8 B$ & 8 & 0 & 0 & 38 & 2398 \\
\hline Kentuchy & 21 & 0 & 2 & + & 0 & 0 & 0 & 2 & $b_{20}$ \\
\hline Louleana & 650 & 59 & 118 & 8白 & 50 & 5 & 10 & 100 & 314 \\
\hline North & 128 & -7 & 35 & 23 & 21 & 0 & 0 & 18 & 136 \\
\hline Sotun Onshore & 382 & 70 & 51 & 49 & 22 & 5 & 6 & 60 & 427 \\
\hline Elate ONfehare & 14日 & -4 & 32 & 14 & 7 & 0 & 4 & 22 & 151 \\
\hline Mictropan & 74 & 5 & 6 & 15 & 7 & 0 & 1 & 10 & 68 \\
\hline Mhstistapen & 184 & 3 & 24 & 10 & 13 & 0 & 7 & 18 & 183 \\
\hline Montaria & 168 & +2 & $\mathbf{B}$ & $\mathbf{B}$ & 5 & 1 & 1 & 15 & 159 \\
\hline Netwagkive & 29 & -3 & 2 & 3 & 0 & 0 & 0 & 3 & $D_{21}$ \\
\hline New Hexkes & 744 & 14 & 84 & 44 & iB & 1 & 1 & 53 & 736 \\
\hline E甲9t & 791 & 8 & 62 & 41 & 18 & 1 & 1 & 81 & 719 \\
\hline Wast & 13 & 6 & 2 & 3 & 0 & 0 & 0 & 2 & 10 \\
\hline North Dahoti & 248 & -3 & 71 & 15 & 12 & 0 & 0 & 34 & 279 \\
\hline Ohro & 53 & -3 & 4 & 6 & 1 & 0 & 0 & 5 & $b_{\Delta 3}$ \\
\hline Oklahoms & 632 & .20 & 100 & 46 & 15 & 0 & 0 & 76 & 605 \\
\hline Pennaynaniale & 10 & 0 & 0 & $\dagger$ & 9 & 0 & 0 & 1 & 17 \\
\hline Texas & 5.736 & 109 & 450 & 325 & 177 & 6 & 8 & 474 & 5,897 \\
\hline RRCC DJștnct $\uparrow$ & 86 & 5 & 4 & 5 & 9 & 0 & 0 & 10 & 89 \\
\hline ARC OIstris 2 Onshore & 63 & 12 & 4 & $d$ & 0 & 0 & 0 & 8 & 66 \\
\hline RAC Disincl 3 Onshort & 291 & .27 & 38 & 24 & 39 & 0 & 0 & 46 & 249 \\
\hline RAC Desinct a Onghore & 51 & 24 & 9 & 14 & 3 & s & 0 & $\mathbf{9}$ & 70 \\
\hline FFC Disunct 5 & 29 & 13 & 22 & 12 & 8 & O & 3 & 9 & 54 \\
\hline RRAC Detinct 6 & 358 & 24 & 16 & 15 & 3 & 0 & 0 & 39 & 34A \\
\hline ARC ONqut 78 & 136 & 6 & 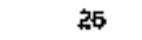 & 6 & 19 & 0 & 0 & 16 & 155 \\
\hline RAC Distnet $7 C$ & $2+9$ & 1 & 23 & 21 & 25 & 0 & 1 & 21 & 297 \\
\hline RAC Dusnet B & 2079 & 21 & 194 & $\$$ & 63 & 0 & 3 & 157 & 2,100 \\
\hline AFG Distnch EA & 2,207 & 12 & 95 & 103 & 14 & 0 & 0 & 127 & 2,099 \\
\hline RFC DभInCI & 144 & 12 & 16 & 15 & 5 & 0 & 1 & 18 & 144 \\
\hline FARC Dnsinet 10 & 74 & 10 & 13 & 12 & 3 & 0 & 0 & 9 & 79 \\
\hline Stake Ofişhqre & 8 & -4 & 2 & 1 & 0 & 0 & 0 & 1 & 4 \\
\hline Ltan & 237 & 9 & 34 & 14 & 1 & 0 & $\mathbf{0}$ & 16 & 234 \\
\hline We日t Virganie & 25 & 4 & 1 & 3 & 1 & 0 & 0 & 2 & 26 \\
\hline wyomung & 803 & 54 & 54 & 26 & 11 & o & 0 & 67 & 627 \\
\hline Féderal Offehore & 3,095 & 4 & 351 & 246 & 90 & 507 & 89 & 397 & 3,477 \\
\hline Pacic (calitomta) & 518 & .24 & 128 & 42 & 2 & 1 & 0 & 55 & 529 \\
\hline Gull of Maxco \{Loulsigna) & 2,357 & 26 & 197 & 176 & 82 & 318 & 81 & 298 & 2,587 \\
\hline Oull of Kanter \{Texas\} & 210 & 2 & 26 & 28 & 6 & $\mathrm{FaB}$ & 2 & $\$ 4$ & 362 \\
\hline Mtescellaniouts ${ }^{a}$ & 18 & 7 & 0 & 5 & 1 & 0 & 0 & 2 & 19 \\
\hline U.S. Totw $\ldots . . . \ldots \ldots \ldots \ldots \ldots$ & 22,017 & 520 & 1,996 & 1,094 & 477 & 697 & 119 & 2,136 & 2,648 \\
\hline
\end{tabular}

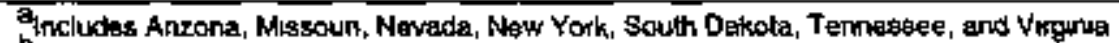

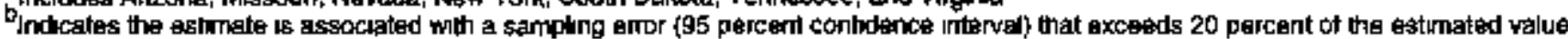
Note. The production estumales un this bable are based on date reported on Fomn E1A-23, “Annual Survey of Dornesbc Oll and Gas

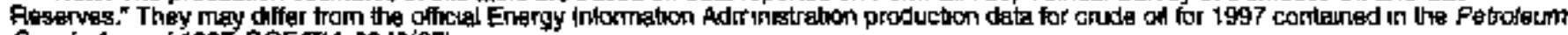
Supply Anjear to97, DOEDEA-0B40 (97)

Scuree Energy Informabon Adminisiration, Ottlee of OA and Gas 


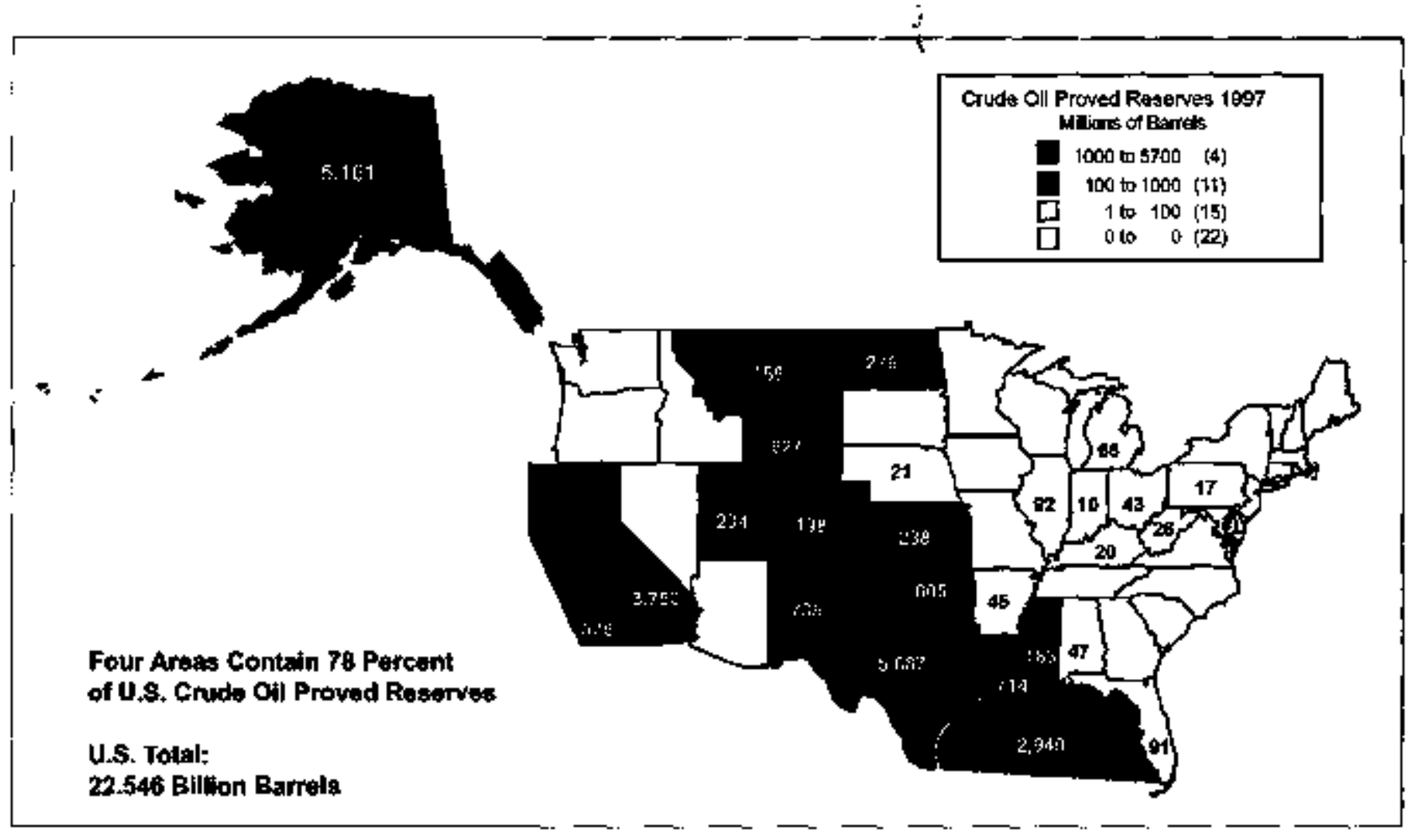

Figure 17. Changea in Crude Oll Proved Reserves by Area, 1996 to 1997

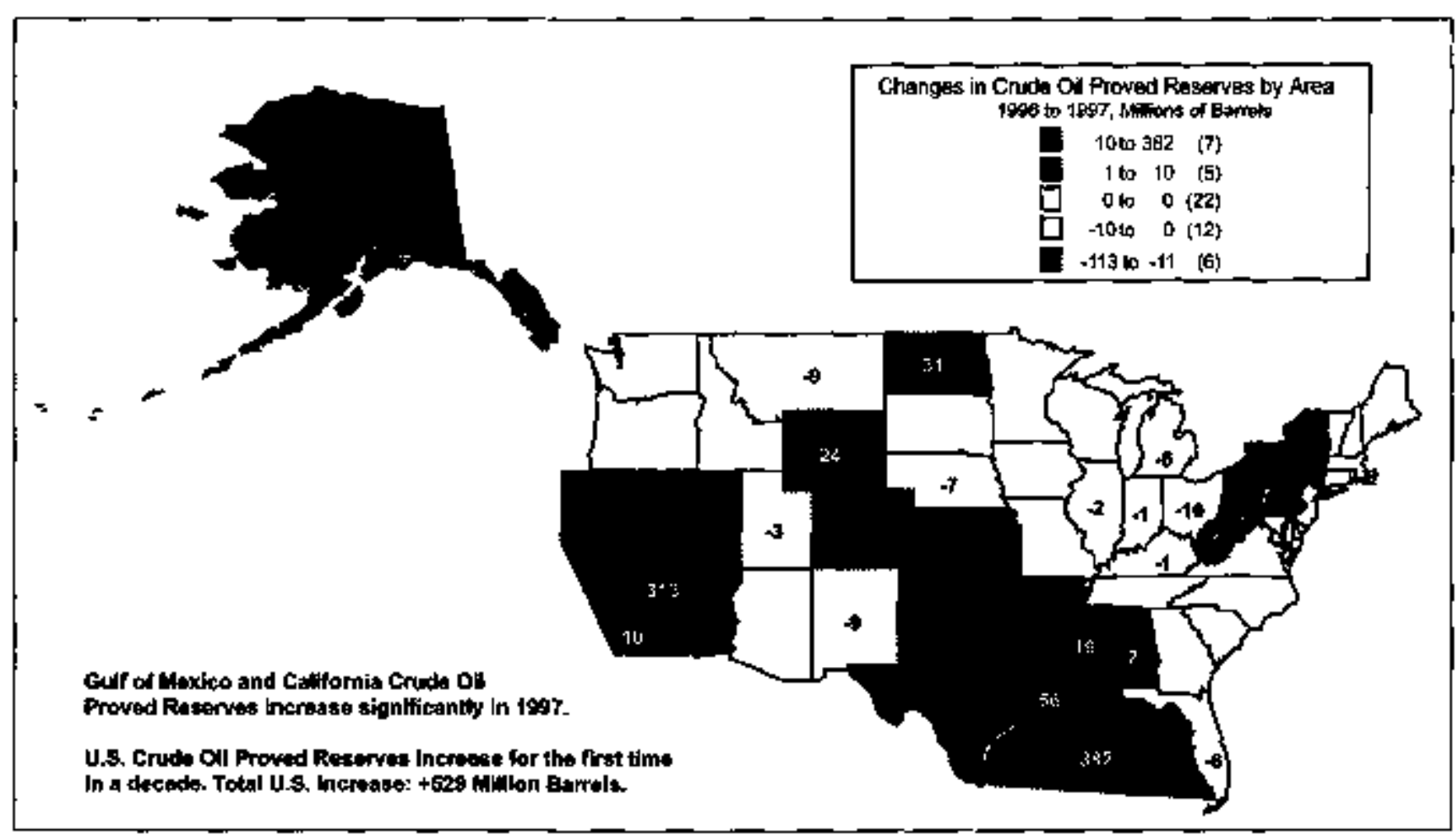

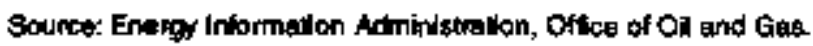


Total discoveries of crude oil were 1,233 million bartsols in 1997, the highest in a decade, and 33 percent hightr than in 1996. Only five areas had tokal discowerie's exceeding 35 million barrels:

- The Gulf of Mexico Federal Offshore had 677 million barrels of total discoveries, 55 percent of the National total.

- Texas had 191 million barrels of total discoveries, 15 percent of the National total.

- Alaska had 135 million barrels of total discoveries, 11 percent of the National total.

- Louisiana had 65 million barrels of total discoteries, 5 percent of the National total.

- California had 46 miltion barrels of total discozeries, 4 percent of the National total.

The United States discovered an average of 693 million barrels of new crude oil proved reserves per year in the prior 10 years (1987 through I996). Total discoperies in 1997 were 178 percent of that average.

\section{Extensions}

Operators reported 477 milljon barrels of exiensions it 1997. The highest volume of extensions was reported i1 Texas (177 million bartels). The Gulf of Mexico Federal Offshore had 88 million barrels of extensions. Louisiana was third with 50 million barrels, followed br California with 38 million barrels.

In the prior 10 years, U.S. operators reported an average of 436 million barrels of exlensions per yeay. The 1997 extensions were 109 percent of that average.

\section{New Fleid Discoveries}

Thesre were 637 million barrels of newo field discoveries reported in 1997. Only eight areas in the United States reported any new field discoteries, and only two contributed more than 1 percent to the total:

- Gulf of Mexico Federal Offshore (79 percent; 50t. million bartels)\}

- Alaska (18 percent; 117 million barrels).

In the prior 10 years, U.S. operators reported an average of 122 million barrel5 of reserves from new field discoveries per year. Reserves from new field discoverie,s in 1997 were more than 5 times that average volume.

\section{New Reservoir Discoveries in Old Flelds}

Operators in the United States reported 119 million barrels of crude oil reserves from new reservoir discoueries in old fields in 1997. As with retw field discoveries, the most significant portion of the new reservoir discoveries in old fields came from the Gulf of Mexico Federal Offshore-83 million barrels or 70 percent of the total. Louisiana had 10 million barrels ( 8 percent), and Califomia and Texas each had 8 million barrels ( 7 percent each). In the prior 10 years, U.S. operators reported an average of 135 million barrels of reserves from new reserwoir discoveries in old fields per year. Reserves from new reserzoir discoveries in old fields in 1997 were 88 percent of that average.

\section{Revisions and Adjustments}

Thousands of positive and negative reotsions to proved reserves occur each year as infill wells are drilled, well performance is analyzed, new technology is applied, or economic conditions change. Adjustments are the annual changes in the ptublished reserve estimates that cannot be directly attributed to the estimates for other reserve change categories because of the survey and statistical estimation methods employed.

There were 1,434 million barrels of net revisions ond adjusments for crude oil in 1997, 57 percent more than in 1996. Califomia alone had 552 million barrels (38 percent) of net revisions and adjuskments in 1997-466 miltion barrels of these were in the San Joaquin Basin Area. Average revisions and adjustments for the prior 10 years were 1,293 million barrels, and those for 1997 were 11 percent higher than this average.

\section{Production}

U.S. production of crude oil in 1997 was 2,138 million barrels. This was 2 percent lower then 1996's total of 2,173 million bartels. U.S. crude oil production has declined in 11 of the last 12 years. Texas and Alaska are still the largest producers of crude oil in the United States with 22 percent of the total each, the Gulf of Mexico Federal Offshore is thind with 16 percent, and California has 13 percent.

\section{Areas of Note: Large Discoveries and Reserves Additions}

The following State and area discussions summarize notable activities during 1997 concerning expected new field reserves, development plans, and possible 
production rates as reported in various trade publications. The citations do not necessarily reflect EIA's concurrence, but are considered important enough to be brought to the reader's attention.

The following areas are the major sucoess stories for crude oil reserves and production for 1997.

\section{Gulf of Mexico Federal Offshore}

Projects in the Gulf of Mexico Federal Offshore were the biggest success stories for oil exploration during 1997. Most of the United States' crude oil reserve additions from new field discoveries and from new reservoir discozeries in old fields came from this area. The net increase of crude oil proved reserves in the Gulf of Mexico Federal Offshore was $\mathbf{3 8 2}$ million barrels. The Gulf of Mexico produced about 342 million barrels of crude oil in 1997, an increase of 13 percent $(39$ million barrels) over 1996's production.

Deepwater Production and Reserves: In 1997 , just as in 1996, major U.S. operators continue to evaluate and develop large prospects in the deepwater areas of the Gulf of Mexico. An example of the newest deepwater technology is shown on the cover of this year's report-an artist's rendering of the installation of the SeaStar platform planned for British-Borneo Petroleum Inc.'s Morpeth Project. Notable fields in the Gulf of Mexico Federal Offshore in 1997 include:

- Hoover-Diana: In the Gtulf of Mexico Federal Offshore (Texas), in East Breaks Block 945, Exxon is developing the Hoover-Diana fields using a deep-draft caisson vessel (ELA also refers to caisson vessels as "spars"), which began construction in Finland in 1997. In the year 2000, Exxon plans to launch the 62,000 ton Hoover caisson vessel and anchor it in 1,570 meters of water. Exxon estimates that Hoover-Diana holds 400 million barrels of oil equivalent. $\{30\}$

- Europa: In the Gulf of Mexico Federal Offshore (Couisiana), in Mississippi Canyon Błock 935. Shell is developing a new oil field in 3,900 feet of water-the Europa Fjeld. Shell and its partners announced in March 1998 their plans to develop Europa utilizing a subsea system capable of accommodating eight wells, tied back 20 miles to the Mars Tension-Leg Platform in Mississippi Canyon Block 807. Total development costs are approximately $\$ 500$ million, excluding lease costs. Production is scheduled to begin in early 2000 and peak production rates are anticipated to be 60,000 barrels of oil per day and $\mathbf{4 5}$ million cubic feet of gas per day by early 2001 . Total gross ultimate recovery is estimated at about 160 million barrels of oil equivalent.|31\}

- Macaroni: In the Gulf of Mexico Federal Offshore (Texas), in Garden Banks Block 602, Shell is developing a new field in 3,700 feet of water-the Macaroni Field. Shell amounced in March 1998 that it would develop Macaroni using a subsea system of three satellite wells clustered around a four-well subsea manifold, tied back 12 miles to the Auger Tension-Leg Platform in Garden Banks Block 426. Total development cost is approximately $\mathbf{5 2 7 0}$ million, excluding lease and exploration costs.

Production is scheduled to begin in mid-year 1999 and peak production rates are anticipated to be 35,000 barrels of oil per day and 65 million cubic feet of gas per day by late 1999. Total gross tultimate recovery is estimated at about 78 million barrels of oil equivalent.\{32\}

- Marlin In the Gulf of Mexico Federal Offshore (Louisiana), in Viosca Knoll Block 915. Amoco is developing the Marlin field in 3,240 feet of water. Amoco and Shell Deepwater Development Inc. announced in January 1997 plans to develop Marlin using a tension leg platform (TLP). Total development cost is approximately $\$ 500$ million. Production is scheduled to begin in mid-year 1999 and peak production rates are anticipated to be 250 million cubic feet of gas and 40,000 barrels of oil daily. Amoco is the operator and hats a 75-percent working interest in the field which is located some 100 miles south of Mobile, Alabana. Construction of the TLP's hull began in 1997 in Taranto, Italy. It will be towed to Corpus Christi, where the topside facilities are being built. The hull will be fastened to the seafloor with steel tendons. [33]

\section{California}

California's proved oil reserves increased by 313 million barrels in 1997. The largest increase was from revisions and adjustments in the San Joaquin Basin Onshore, home of Califomia's "heavy oil" fields. This area's reserves increased by 274 million bayrels. Californiats production also increased by about 3 percent from 1996's level.

A key player in Califorria's heavy oil industry is Texaco, lnc., which contributed to the surge of proved 
reserves in California by acquiring Monterey Resources, a California independent oil and $g$ ss producer, in November 1997. Texaco expects that application of the continuous-injection steam flood process it used at its Kern River Field, in comparison to Mfonterey's less efficient cyclic steam flood procets, will raise production while simultaneously lowering per-barrel production costs. Over the next 3 years, Texaco's strategic goal is to more than double production from formier Monterey Resources fields to 119,000 barrels of oil equivalent per day.\{34\}

\section{Texas}

Onshore in the lower 48 States, Texas had the most tot 1 discnoeries of crude oil proved reserves in 1997 (131 million barrels). Enhancements in drilling technology, coupled with improved economics (due to reductions in development well costs and higher oil prices), caused increased operator activity.

\section{Alaska}

Alaska reported 135 million barrels of total discoteries of crude oil proved reserves in 1997, third in the Nation following the Gulf of Mexico Federal Offshore artd Texas. Most of Alaska's total discoperies were from new fields on the North Slope. These tields are "satellite fields ${ }^{\prime \prime}$, meaning that they are located next to large, existing Alaskan North Slope fields and can be developed economically by extending facilities from the existing production sites to reach them.

In Jate December 1997, ARCO began commercial production from West Sak, a heavy oll accumulation. Commercial development of West Sak has become feasible because of lower costs, new drillirg technologies, and a production facility sharing agreement with the Kuparuk production facility. The first phase of West Sak development has resulted in proved gross reserves of 50 million barrels of oil (25 net to ARCO). ARCO also had success drilling in a number of satellite accumulations, including the Tarn field. $\{35\}$

\section{Louisiana}

Louisiana's proved oil reserves increased by $56 \mathrm{million}$ barrels. The largest increase was from revistons and adjusiments in the South Onshore region. Loursiana's production also increased by about 4 percent from 1996's level.

\section{Other Gain Areas}

North Dakota: North Dakota's proved oil reserves increased by 31 million barrels. There were 53 million barsels of net rezisions and adjustmenls in North Dakota in 1997. plus 12 million barrels of extensions.

Wyoming: Wyoming's proved oil reser:os increased by 24 million barrels. Although there were no new fields reported, several operators increased their reserves with extensions and revisions and adjustments.

Mississippi: Mississippi's proved oil reserves lncreased by 19 miltion bartels. The boost came from a combination of extensions, revisions and adjustments, and new reserwoirs discovered in old fields.

\section{Areas of Note: Large Reserves Declines}

The following areas had lange declines in crude oil proved reserves due to downward revisions or unreplaced production.

\section{Alaska}

Alaska's proved oil reserves declined by 113 million barrels in 1997-the greatest decline in any state. This is because Alaska's massive production rate far exceeds its reserves replacement rate. Alaska's 1997 production was approximately 473 million barrels, 7 percent less than what Alaska produced in 1996.

No Production Decline in 1999: In 1997, ARCO started several projects and had several successes that it believes will stop the decline in its Alaskan production after 1999. The Prudhoe Bay Miscible Injection Expansion ("MIX") Project was approved by ARCO and its partners. This $\$ 160$ million exparsion of the gas handling facilities is expected to add 50 million gross barrels of petroleum liquids and 20 thousand net barrels of petroleum liquids per day to the field when it becomes operational, targeted for 1999.(35)

\section{Texas}

Texas' proved oil reserves declined by 49 million barrels in 1997. Texas' production also declined about 4 percent from 1996 levels. The largest reserves decline (109 million barrels) was in RRC District 8A. In this district, the net revisions and extensions were exceeded by production. Other areas where oil reserves significantly decreased were RRC District 3 Onshore 
(22 million barrels) and RRC District 6 (11 million barrels).

These declines were counterbalanced somewhat by reserves increases in other RRC Districts. In RRC Districts 5 and $B$, net positive reserves additions of 25 and 21 million barrels of proved reserves, respectively, were added.

\section{Other Decllne Areas}

In the following areas of the United States, development of existing or new oil fields was outpaced by cude oil production, which remained at roughly the same level as the previous year.

Colorado: Proved oil reserves decreased by 14 percent ( 33 million barrels).

Kansas: Proved oil reserves decreased by 11 percent (28 million barrels).

Oklahoma: Proved oil reserves decreased by 4 percent (27 million barrels).

\section{Reserves in Nonproducing Reservoirs}

Not all proved reserves of crude oil were contained in reservoirs that were producing. Operators reported 3,755 million barrels of proved reserves in nonproducing reservoirs, 8 percent more than reported in 1996 (3,474 million barrels).

The reasons for the nonproducing status of these proved reserves are not collected by the EIA. However, previous surveys showed that most were not being produced for operational reasons. These included waiting for well workovers, drilling additional development or replacement wells, installing production or pipeline facilities, and awaiting depletion of other zones or reservoirs before recompletion in reservoirs not currently open to production

\section{Indicated Additional Reserves}

In addition to proved reserves of crude oil, Category 1 and Category IJ operators estimate the quantities of crude oil, other than proved reserves, that may become economically recoverable from known reservoirs through the application of improved recovery techniques using currently available technology. The 1997 volume, 3,207 million barrels, is about 12 percent more than was reported in $1996(2,876$ million barrels $)$.

Table 7 lists the indicated additional reserves by selected States and State subdivisions. The presence of large indicated additional reserves in Alaska, California, south Lowișiand and west Texas implies that significant upward revisions to proved crude oil reserves could occur in the future. 
Table 7. Reported Indicated Additional Crude Oil Reserves, ${ }^{2} 1997$ (Milion Barrels of 42 U S. Gallons)

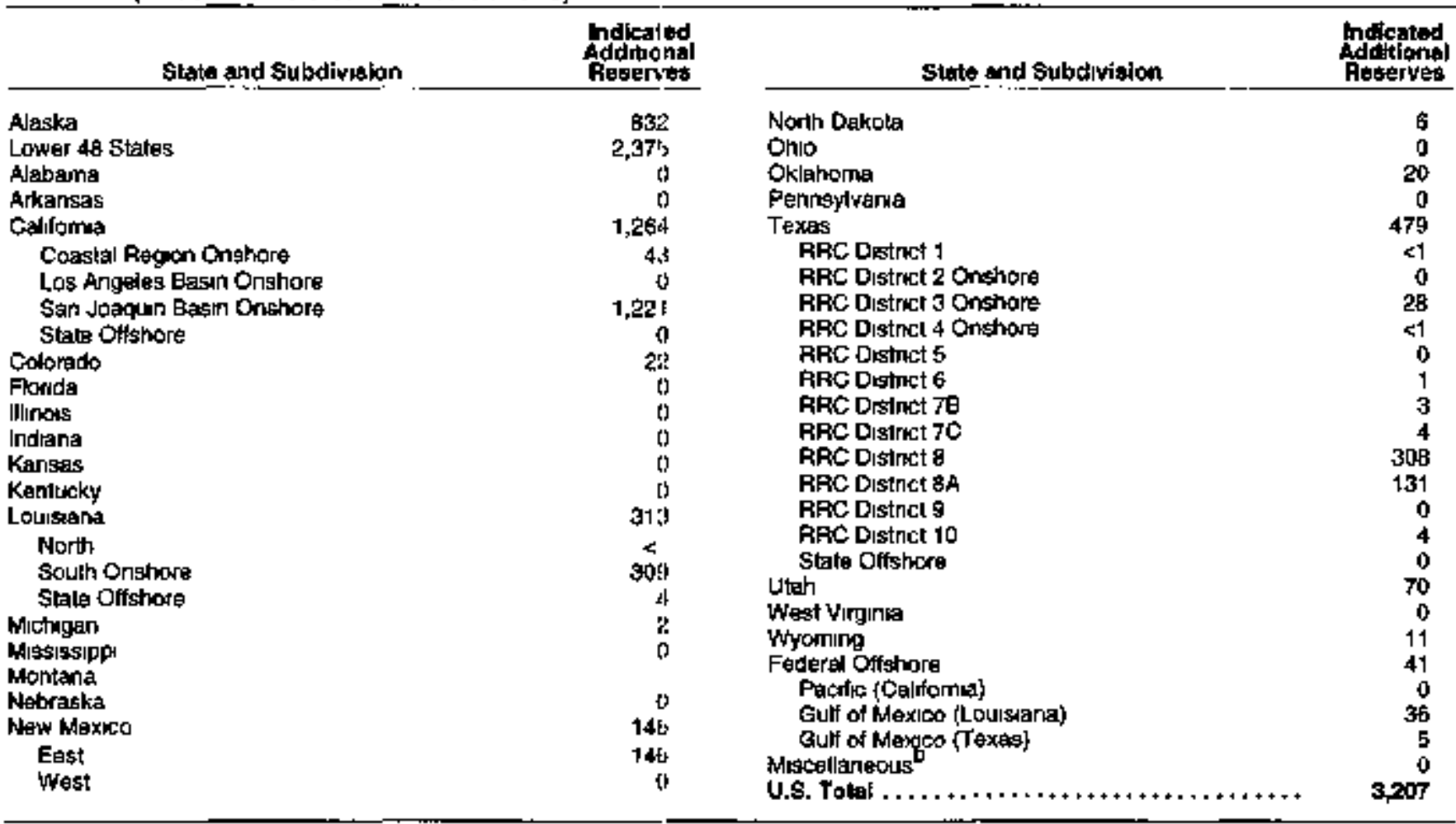

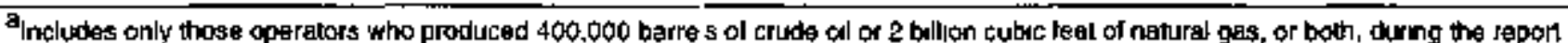
year (Calegory I or Calegory li operators')

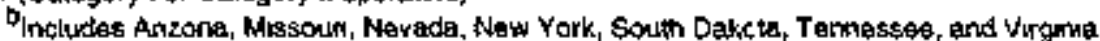

Source Fom ElA-23, 'Annuel Sunvey of Domestre On and Ras Reserves, 1997 


\section{Natural Gas Statistics}

\section{Dry Natural Gas}

\section{Proved Reserves}

The Nation's 1997 proved reserves of dry natural gas were 167,223 billion cubic feet, 0.4 percent ( 749 billion cubic feet) more than in 1996 (Table $B$ ). This is the fourth consecutive year that natural gas reserves have increased. Gas production increased for the second consecutive year.

Additions to reserves replaced 104 percent of gas production in 1997 (Figure 18). New gas discoveries in the Gulf of Mexjco Federal Offshore, Texas, Wyoming, and Louistana, combined with upward revisions of gas reserves in Alaska and Oklahoma, were responsible for this. Lower $\mathbf{4 8}$ States gas reserves declined slightly in 1997 after increasing in 1995 and 1996. The decrease in the Lower 48 in 1997 was more than made up for by the Alaskan gas reserves additions.

Figure 18. Reserve Additlons Exceed U.S. Natural Gas Production 4 Years in a Row

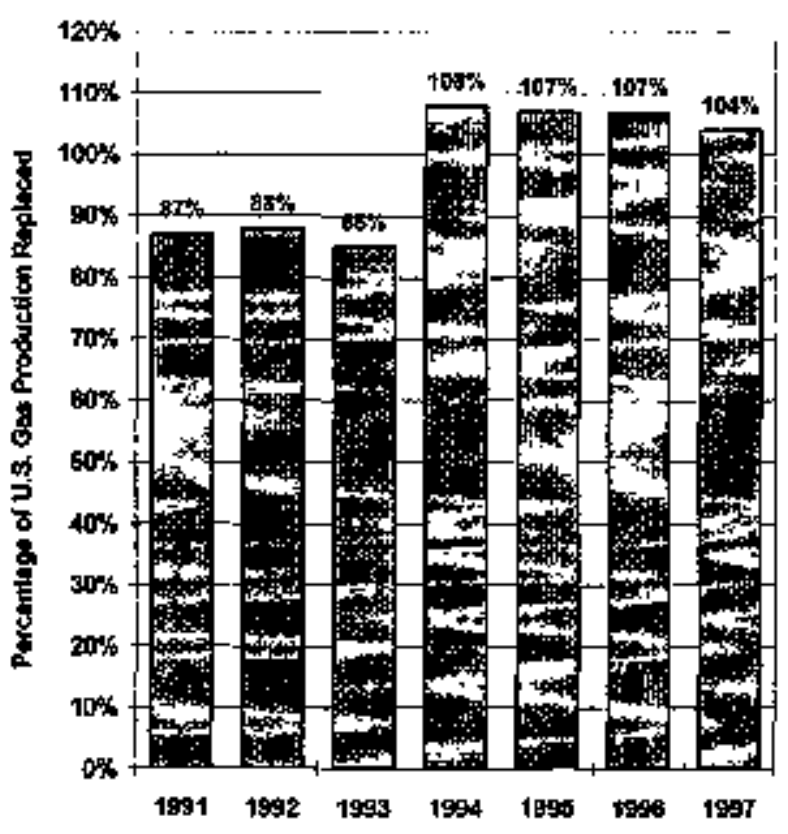

Source- Energy Hormation Administratron, Office of OA and Gas.

Proved reserves by State are shown on the map in Figure 19. Six areas account for 71 percent of the Nation's dry natural gas proved reserves:
Percent of U.S. Gas Peserves

\begin{tabular}{lr} 
Area & U.S. Gas R \\
\hline Texas & 23 \\
Gulf of Mexico Federal Offshore & 17 \\
New Mexico & 9 \\
Wyoming & 8 \\
Oklahoma & 8 \\
Alaska & 6 \\
Area Total & 71
\end{tabular}

Of these six areas, the Gulf of Mexico Federal Offshore, Alaska, Wyoming, and Oklahoma had increased reserves in 1997, while Texas and New Mexico had decreases in dry natural gas proved reserves.

\section{Discussion of Reserves Changes}

Figure $\mathbf{2 0}$ maps the change in dry gas proved reserves from 1996 to 1997 by area. Here's how the top six areas fared, compared to the total United States:

Chenge in U.5. Gas Reserves

\begin{tabular}{lr} 
Area & (billon cubic \\
\hline Texas & -509 \\
Gutf of Mexico Federal Otishore & +70 \\
New Mexico & -971 \\
Wyoming & $+1,242$ \\
Otdahoma & +365 \\
Alaska & $+1,268$ \\
Area Total & $+1,465$ \\
U.S. Total & +749
\end{tabular}

Figure 4 in Chapter 2 shows the components of the changes in dry natural gas proved reserves for 1997 and the preceding 10 years.

\section{Revisions and Ad]ustments}

Reossions and adjustmenis declined $\mathbf{4 5}$ percent in 1997. compared to 1996's level. The net volume of revisions and adjustments was 4,312 billion cubic feet. Alaska had the largest increase in revisions and adjustments (1,729 billion cubic feet). Alaska actually has substantially greater total resources of naturial gas than proved reserves, but no economic market presently exists for Alaskan gas. 
Table 8. Dry Natural Gas Proved Reserwes, Reserves Changes, and Production, 1997 (Billion Cubic Feet at 14.73 psia and $60^{\circ}$ Fahrenhert)

\begin{tabular}{|c|c|c|c|c|c|c|c|c|c|}
\hline \multirow[b]{2}{*}{ statu ind Subdrivion } & \multirow[b]{2}{*}{$\begin{array}{l}\text { Publushed } \\
\text { Prowed } \\
\text { Puberve: } \\
\text { 12/31/is } 6\end{array}$} & \multicolumn{7}{|c|}{ Changas in Feserva During 1987} & \multirow[b]{2}{*}{$\begin{array}{l}\text { Prowed } \\
\text { Peserves } \\
128197 \\
\end{array}$} \\
\hline & & $\underset{(\bullet,-)}{\operatorname{Adj}}$ & $\begin{array}{c}\text { Powtion } \\
\text { incroiate: } \\
{[+]}\end{array}$ & 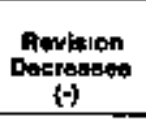 & $\begin{array}{c}\text { Extergadons } \\
1+\}\end{array}$ & $\begin{array}{c}\text { Maw Fiold } \\
\text { Diacoverdes } \\
(+)\end{array}$ & $\begin{array}{l}\text { Raw Raserveir } \\
\text { Discowerlos: } \\
\text { In old Fields } \\
\text { (+) }\end{array}$ & $\begin{array}{c}\text { Erilimated } \\
\text { Productian } \\
\{t\end{array}$ & \\
\hline Alaska & 9.294 & 19 & $1, \mathrm{BOO}$ & 99 & $1 d$ & 0 & 0 & 475 & 10592 \\
\hline Lower 40 statip . . . . . . . . . & $-167,180$ & 609 & 19,849 & 19,667 & 16,5T1 & 2,691 & 2,392 & 19,756 & 168,661 \\
\hline Alabame & 5,030 & .31 & 348 & 54 & 3d & 0 & 14 & 376 & s geb \\
\hline Arkansag & 1470 & 21 & 205 & 101 & 86 & 0 & 15 & 199 & 1475 \\
\hline Celloma & $2,0 \mathrm{A2}$ & 18 & 350 & 52 & 68 & 0 & 10 & 212 & 2,273 \\
\hline Cogstal Regran Onghorg & 156 & 18 & 10 & 10 & 15 & 0 & $\mathbf{1}$ & 17 & 164 \\
\hline Lcts Angolas Bann Orathere & 109 & 21 & 27 & 6 & 0 & 0 & 0 & 10 & 141 \\
\hline San Jorann Bagn Denshore & 1.706 & -31 & 318 & 27 & 53 & 0 & 9 & 178 & 1.912 \\
\hline SÁle Olishore & 49 & 11 & 3 & 0 & D & 0 & 0 & 7 & 56 \\
\hline Colorado & 7.710 & -563 & 959 & 的B & 108 & 2 & 0 & \$0द & 6.828 \\
\hline Fonda & 96 & 5 & 1 & 0 & 0 & 0 & 0 & B & 98 \\
\hline Kangas & 7,694 & $\theta 0$ & 307 & 510 & 54 & $D$ & 4 & top & 0.99 \\
\hline Kenturiky & 983 & 321 & 107 & 3 & 18 & 0 & 17 & 79 & 1364 \\
\hline Loukgena & 8543 & 203 & 1808 & $1,3+\infty$ & 754 & 11 & 412 & 1,490 & 0.673 \\
\hline North & 3.705 & B & 607 & 378 & 175 & 0 & 2 & $42 \$$ & 3,093 \\
\hline South Onshore & 5.704 & 157 & 897 & esse & 648 & 11 & 318 & 911 & $\$, 4 \$ 5$ \\
\hline State Oftshore & 734 & 40 & $1+2$ & 123 & 31 & 0 & 92 & +61 & 725 \\
\hline Muhigen & $20 \$ 1$ & 177 & 252 & 124 & 50 & 0 & 0 & 221 & 2185 \\
\hline Mrsagssops & 831 & .18 & 86 & 58 & 48 & 0 & 2 & 93 & 582 \\
\hline Mondang & 798 & 93 & 99 & t\$9 & 22 & 0 & 0 & 55 & కే6z \\
\hline New Mexoca & 16,485 & -664 & 2,459 & 1,868 & 641 & 2 & 6 & 1,547 & 15,514 \\
\hline Eas! & 2,790 & 12 & 392 & 334 & 182 & 2 & $\mathbf{6}$ & 418 & 2,642 \\
\hline Wota & 13,695 & 676 & 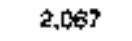 & 1,534 & 446 & 0 & 0 & 1.120 & 12,972 \\
\hline Naw York & 232 & -12 & $\infty$ & 42 & 0 & D & 4 & $c_{18}$ & ${ }^{\mathrm{C}_{224}}$ \\
\hline Noth Dakcka & 462 & -2 & 57 & 17 & 25 & 0 & 0 & 46 & 479 \\
\hline Ohes & 1,713 & 71 & 101 & 75 & 5 & 0 & 17 & 105 & 985 \\
\hline Oklahoms & 13,074 & 82 & 2395 & 1.982 & 639 & 2 & 84 & 1,595 & 13439 \\
\hline Pentesymania & 1896 & EO & 510 & 391 & 82 & 0 & 5 & 129 & 1852 \\
\hline Texas & 30,270 & -495 & $4,6 \in 0$ & 4,447 & 3,949 & 373 & 396 & 4,942 & 97761 \\
\hline RAC Doglnet t & 905 & 自5 & $11 \mathrm{~B}$ & 55 & 149 & 25 & 0 & 105 & $\$ 53$ \\
\hline RRC Dusthet 2 Onshore & 1.322 & 102 & 248 & 200 & 305 & 50 & 30 & 214 & 1694 \\
\hline AFC Duslnct S Onghare & 4,349 & -\$\$\$10 & 784 & 505 & 488 & 193 & 59 & Bag & 4172 \\
\hline FRC Dsinct 4 Onshore & 7,769 & 57 & 1,155 & $t, 179$ & 1,452 & 32 & 105 & 1,292 & B 099 \\
\hline ARC Dustnct 5 & 2070 & 的 & 163 & 808 & 69 & 25 & 152 & $2+0$ & 1,710 \\
\hline ARC Destnct $\theta$ & 5,099 & $1+2$ & 503 & 592 & 583 & 0 & 11 & 599 & 5887 \\
\hline RRC Dustngt T日 & $\$ 200$ & -110 & 105 & 40 & 46 & 23 & 0 & 自? & 478 \\
\hline ARE Drstnct 7C & 3,655 & $\$ 7$ & 305 & 358 & 248 & 4 & 10 & 372 & 3,407 \\
\hline FRC Destrict 8 & 5,459 & $\cdot 210$ & 754 & 373 & $\$ 16$ & $B$ & 22 & 572 & 5,398 \\
\hline RAC D-sinct BA & $\$ 31$ & -22 & 35 & 54 & 12 & 0 & 0 & 55 & 847 \\
\hline FRE Drझnct 9 & 705 & 110 & 47 & 74 & 111 & 0 & $\mathbf{1}$ & 100 & 794 \\
\hline RAC Dranct 10 & 4,391 & -142 & 384 & 340 & 189 & 2 & 3 & 387 & 4094 \\
\hline State Ontshore & 292 & 22 & $\mathbf{5 6}$ & 34 & 7 & 11 & D & 77 & 289 \\
\hline Utah & 1,639 & -40 & 293 & 34 & 164 & 0 & 0 & 177 & 1839 \\
\hline Virģınıa & 1,930 & 344 & 142 & 15 & 113 & 0 & 0 & 68 & 2446 \\
\hline West Virgmila & 2,709 & 182 & 156 & 80 & 12 & 0 & 40 & 167 & 2,946 \\
\hline Wyoming & 12,320 & 207 & 1191 & 1,988 & 2,047 & 39 & 17 & B91 & 13,562 \\
\hline Fedorel onsthorea & 20,096 & .390 & 3486 & 3,8006 & 1, Bds & 2,252 & 1,342 & 5,170 & 28,466 \\
\hline Pacsic (Catiforrug) & 1,244 & 自守 & 6 & 602 & 1 & 1 & 0 & 37 & 544 \\
\hline Gull al hexoo (Loulsana ${ }^{a}$ & 21,958 & 390 & 2.705 & 2,250 & 1,240 & 1,511 & 1.151 & 3,995 & 21,934 \\
\hline Gull of hlaxocs (Tix日a) & 5,996 & 79 & 775 & 951 & 398 & 740 & 191 & 1,238 & 5, 距 \\
\hline Anscellangous & 67 & $\cdot 16$ & 21 & 26 & 0 & 0 & 0 & $5_{3}$ & $0_{43}$ \\
\hline U.8, Total $, \ldots \ldots, \ldots, \ldots$ & 168,474 & -590 & 21,668 & 16,758 & 10,585 & 2,661 & 2,392 & 19,211 & 167,293 \\
\hline
\end{tabular}

Iincludas Faderal otlshore Alaberna

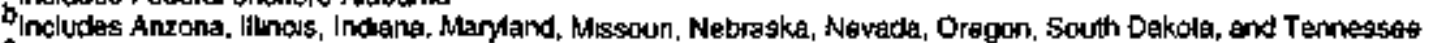

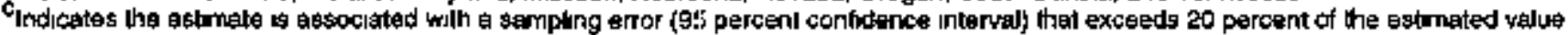

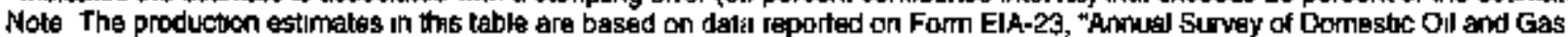

Pejeerves," end Folm ElA-64A, "Annuel Report of the Owgin of Natural Gas Liquads Produchon "They may differ from the offickal Energy

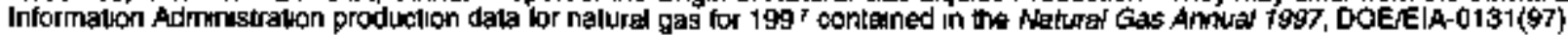
Souree Ensrgy informaton Authenistration, Office ol Oll and cias 


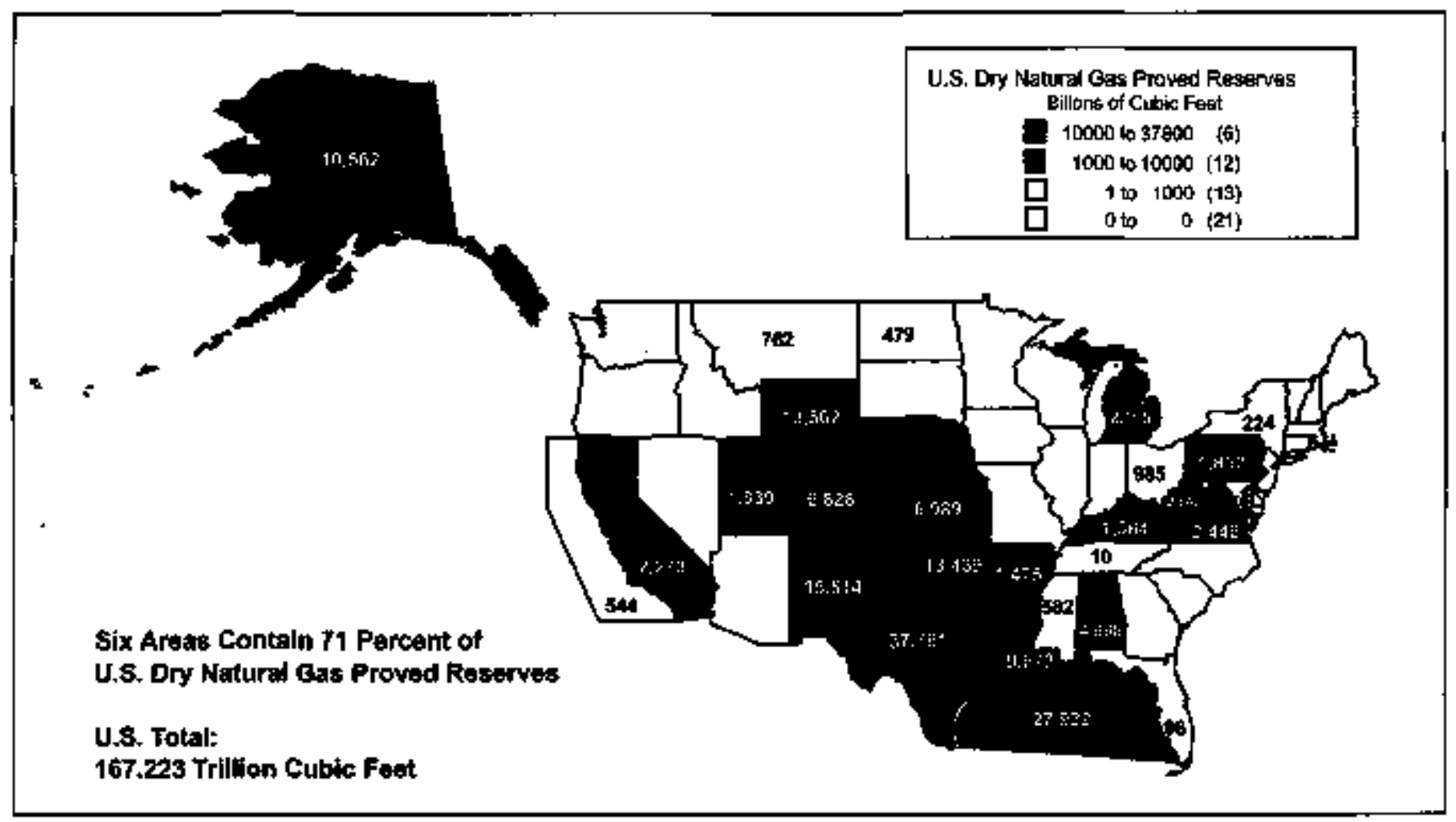

Figure 20. Chenges in Dry Natural Gas Proved Feaerves by Area, 1986 to 1997

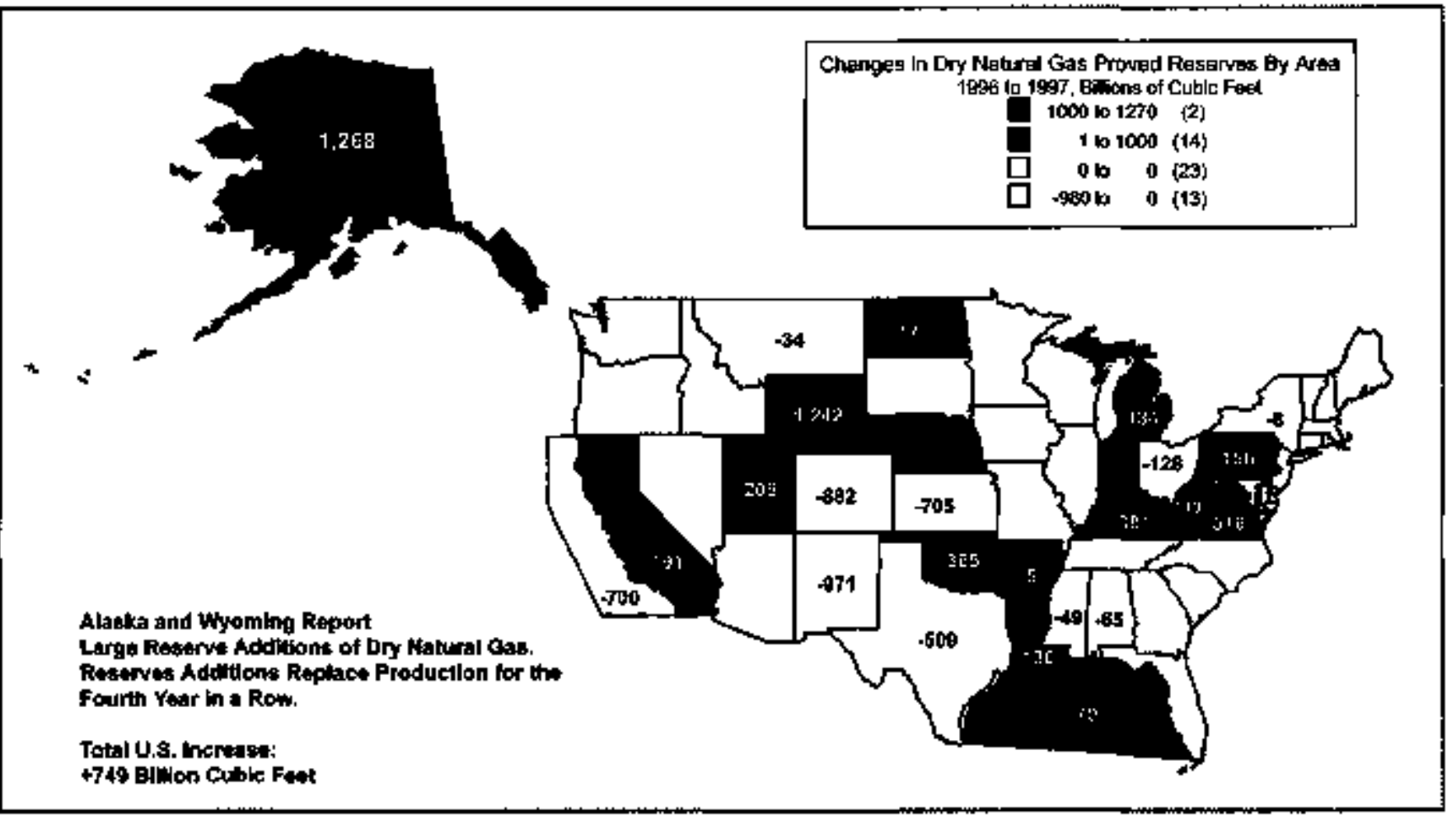

Source: Enangy Intarmation Administration, Othco of OA and Gas. 


\section{Discoveries}

Total discoutries were up from 1996. Total discoveries are those reserves attributable to field extensions, new fietd discoueries, and new reservoir discorderies in old fields; th ey result from drilling exploratory wells. Total discoveries of dry natural gas reserves were 15,648 billion cusic feet, an increase of 27 percent from the level reported in 1996 and equivalent to 81 percent of 1997 हas production. About 64 percent of the total discoveries were in the Gulf of Mexico Federal Offshore and Texis.

Extensions were 10,585 billion cubic feet, 36 percent higher than in 1996. Areas with the largest extensions were:

- Texas ( 37 percent)

- Wyoming (19 percent)

- Gulf of Mexico Federal Offshore (16 percent)

- Louisiana (7 percent)

- New Mexico (6 percent)

- OkJahoma (6 percent).

In the prior 10 years, U.S. operators reported an average of 6,309 bilion cubic feet of reserves from extersions per year. Reserves from extensions in 1997 were 68 percent higher than that ayerage volume.

New field discoveries were 2,681 billion cubic teet-85 percent higher than in 1996. Those areas with the largest new field discoweries were the Gulf of Mexico Federal Offshore (with 84 percent of the total) and Texas (14 percent). In the prior 10 years, U.S. operators reported an average of 1,359 bilition cubic feet of reserves from new feld discoteries per year. Reserves from new field discozeries in 1997 were almost wice that.

New reservoir discoveries in old fields were 2,382 billion cubic feet, 23 percent lower than 1996. Among the areas with the largest new reservoir disconeries in old fields were:

- Gulf of Mexico Federal Offshore (56 percent)

- Louisizna (17 percent)

- Texas ( 16 percent).

In the prior 10 years, U.S. operators reported an average of 2,230 billion cubic feet of reserves from new reserwoirs discovered in old fields per year. Reserves from new reserooirs discovered in old fields in 1997 wers 7 percent higher than that average volume.

\section{Production}

Dry natural gas production increased 2 percent in 1997 (Table 8). U.S. gas production has increased during 8 of the last 10 years. As in 1996, the Gulf of Mexico Federal Offshore and the State of Texas were the leading producers of dry natural gas in 1997, each with over one-fourth of the U.S. total. The next three States combined, Oklahoma (8 percent), Louisiana (8 percent), and New Mexico (s percent), added another one-fourth of the production.

\section{Wet Natural Gas}

U. S. proved reserves of wet natural gas, as of December 31, 1997, were 175,721 billion cubic feet, an increase of 0.3 percent, or 574 billion cubic feet, from that reported in 1996 (Table 9). At year-end 1997 proved wet natural gas reserves for the lower 48 States had decreased by 0.5 percent ( 803 billion cubic feet) compared to 1996, while those of Alaska had increased by 15 percent ( 1,377 billion cubic feet).

The volumetric differences between the estimates reported in Table 8 (dry) and Table 9 (wet) results from the removal of natural gas liquids at natural gas processing plants. A discussion of the methodology used in this report is in found in Appendix F. Alt natural gas proved reserves data shown in this report exclude natural gas held in underground storage.

\section{Nonassoclated Natural Gas}

\section{Proved Reserves}

Proved reserves of nonassociated (NA) natural gas, wet after lease separation, in the United States decreased by 0.7 percent (1,013 billion cubic feet) in 1997 to 143,339 billion cubic feet (Table 10). The lower 48 States' NA wet natural gas proved reserves decreased by 0.5 percent ( 754 billion cubic feet). Those areas with the largest increases in NA wet natural gas feserves were Wyoming. Texas RRC District 1, and Kentucky. There were large decreases in NA wet natural gas reserves in New Mexico, the Gulf of Mexico Federal Offshore, and Kansas.

\section{Discoveries}

NA wet natural gas total discoperies of 13,718 billion cubic feet increased 21 percent $(2,380$ billion cubic feet) in 1997. Texas, the Gulf of Mexico Federal Offshore, 
Table 9. Naturel Gas Proved Roserves, Reserves Changes, and Production, Wet After Lease Separation, 1997 (Billion Cubic Feet at $14.73 \mathrm{ps}$ la and $60^{\circ}$ Fahrenhert)

\begin{tabular}{|c|c|c|c|c|c|c|c|c|c|c|c|}
\hline \multirow[b]{2}{*}{ 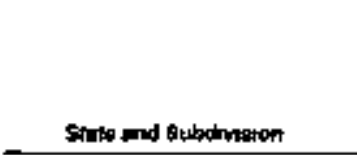 } & \multirow[b]{2}{*}{ 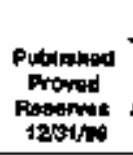 } & \multicolumn{6}{|c|}{ Changes in Fetarveg Dunng 1097} & \multicolumn{4}{|c|}{ 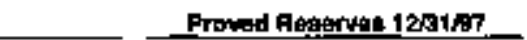 } \\
\hline & & Adjuetonente & 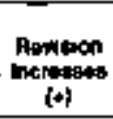 & 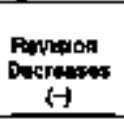 & $\begin{array}{c}\text { Extonemons } \\
\{+\}\end{array}$ & 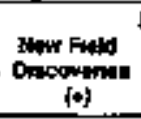 & 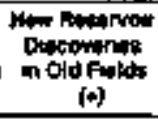 & $\begin{array}{c}\text { Producikon } \\
(-1)\end{array}$ & Totw Gid & $\begin{array}{l}\text { Non- } \\
\text { ansocinied } \\
\text { Eien }\end{array}$ & 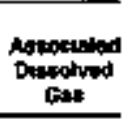 \\
\hline Alaskika & 9,575 & 69 & 202 & 171 & a & 81 & D & 448 & 9,296 & 3,216 & 6,0 \\
\hline Lower da sanies ..., .......... & 180,901 & 4,219 & 17,083 & 13,686 & 9,183 & $1,4 \$ 0$ & 3,200 & 10,350 & 164,861 & 141,136 & 24,745 \\
\hline Alabami & 4,930 & $-\mathbf{d \phi}$ & 507 & 97 & 177 & 0 & 0 & 37 & 5,100 & 5,062 & 38 \\
\hline Aukantas & 1,566 & 32 & 97 & 100 & 57 & 2 & 27 & 201 & 1,472 & 1,363 & 的 \\
\hline Caliontiva & 2,355 & -111 & 217 & 74 & 18 & 0 & 19 & 228 & 2,193 & 610 & 1,586 \\
\hline Coaslal Regionn Onghore & 168 & 11 & 17 & 10 & 1 & 0 & 0 & 14 & 168 & 48 & 120 \\
\hline Los Angejes Be日n Ontshore & 115 & -4 & 16 & 5 & 0 & o & 0 & 10 & 112 & 0 & 412 \\
\hline San Jaquin Besin Onenore & 2,018 & -118 & 193 & 50 & 15 & 0 & 13 & 190 & $1, \mathrm{~B} \times 44$ & 500 & 1,304 \\
\hline Stats Oftshor: & 59 & 0 & 1 & 3 & 0 & 0 & 0 & B & 49 & 2 & 47 \\
\hline Colowado & 7,592 & 49 & $\$ \$ \$ 4$ & 428 & 112 & 0 & 5 & 564 & 8,064 & 7,009 & 1.085 \\
\hline Flonda & 110 & 0 & 15 & 0 & 0 & 0 & 0 & 6 & 110 & 0 & 110 \\
\hline Kansas & 9,093 & 310 & 399 & 1,000 & 62 & 0 & 3 & 744 & 9,145 & $8,06,3$ & 82 \\
\hline Kentuxity & 1,102 & -49 & 45 & 12 & 11 & 1 & 10 & 的 & 1,046 & 1,022 & 24 \\
\hline Louletane & 0,891 & 577 & 1,168 & 1,036 & 804 & 14 & 470 & 1.611 & 10,077 & 9,038 & 1,009 \\
\hline North & 2,863 & 145 & 496 & 170 & 256 & 4 & 10 & 397 & 3,169 & 2,934 & 255 \\
\hline South Onshore & 6,166 & 401 & 672 & 755 & $\$ 17$ & 10 & 410 & $t, 0<5$ & 6,105 & $5,47 \mathrm{~B}$ & 627 \\
\hline Shale Otlshore & 882 & 31 & 110 & 103 & 31 & 0 & 41 & 189 & 783 & 56 & 157 \\
\hline Michıgan & 1,344 & $\operatorname{sig} 9$ & 487 & 158 & 18 & $7 \theta$ & 0 & 215 & 2,125 & 1.778 & 347 \\
\hline Misgespipp & 667 & 13 & 87 & 89 & 43 & 0 & 1 & $\Leftrightarrow$ & 634 & 587 & 47 \\
\hline Monlana & 792 & 17 & 59 & 12 & 2 & 0 & 1 & $\$ 0$ & 806 & 755 & 51 \\
\hline New Manco & 18,747 & 158 & 1,157 & $1,22 z$ & BOP & 4 & 0 & $1,5 \$ 0$ & 17, 媓5 & 16,232 & 1,690 \\
\hline East & 3,207 & 25 & BOS & 219 & 160 & 4 & $\theta$ & 484 & 3,107 & 1,612 & 1,585 \\
\hline West & 15.540 & 133 & 655 & 1004 & 412 & 0 & 0 & 1,086 & 14,728 & 14,020 & 108 \\
\hline Natw York & 197 & 20 & 30 & 12 & 3 & 0 & 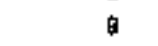 & 21 & 2932 & 229 & s \\
\hline North Daketa & 518 & $1 \mathrm{~s}$ & 41 & 25 & 官 & 3 & 0 & 50 & 512 & 257 & 255 \\
\hline Onio & 1,054 & 102 & 91 & 42 & 0 & 0 & 30 & 121 & 1,114 & tis & 390 \\
\hline Coklathoma & 14,295 & 176 & 2,013 & 1.710 & 792 & 32 & 70 & 1,688 & 13,062 & 12,929 & 1,023 \\
\hline Penningyluanata & 1,488 & 138 & 244 & 103 & 38 & 0 & 29 & 132 & 1,702 & 1,655 & 47 \\
\hline Texas & 39,736 & 1.175 & 5,037 & 3,719 & 3,445 & 543 & 682 & 5,287 & 41,692 & 3432 & 8110 \\
\hline FAC Drstnea t & 7AE & -24 & 225 & 39 & 152 & 2 & 0 & 93 & 969 & 523 & 446 \\
\hline RAC Destna 2 Onsthore & 1,371 & 120 & 198 & 227 & 143 & 0 & 40 & 2008 & 1,430 & 1,178 & 252 \\
\hline RAC Destnch 3 Orsshpres & 4,156 & 290 & 560 & 535 & 570 & 181 & 938 & 916 & 4,052 & 3,612 & 1.040 \\
\hline RAC Desinct 4 Onshore & 8.021 & 139 & 1,129 & 7.191 & 1.023 & 99 & 231 & 1,321 & 8,123 & 7,877 & 246 \\
\hline RAC Desinc1 5 & 1,923 & 60 & 146 & 136 & 94 & 233 & B & 193 & 2,141 & $2, \cos 8$ & 53 \\
\hline AFC Drstnet E & 0,006 & 166 & 647 & 267 & 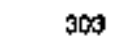 & 0 & 5 & 618 & 6.252 & 5,600 & sa? \\
\hline RRC, Distrin Te & 599 & 52 & 142 & en & 12 & o & $\theta$ & 81 & 610 & 378 & 232 \\
\hline RRG Disknea TC & 3,468 & 45 & 649 & 176 & 477 & 0 & 12 & 412 & 4,063 & 3.371 & 的是 \\
\hline FAC DIgtnG 8 & 6,052 & 342 & 606 & 603 & 272 & 16 & 11 & 846 & 6,050 & 3009 & 2, \\
\hline AAC Disinct BA & 1,333 & 6 & 89 & 45 & B & 0 & 0 & 97 & 1,290 & 18 & 1,276 \\
\hline AAC D.STnCA & 888 & $?$ & 65 & 31 & 89 & 0 & 0 & 128 & 870 & 749 & 121 \\
\hline RAC DIStongt 10 & 4.970 & -20 & sso & 369 & 259 & $\uparrow 2$ & 7 & 304 & 4,845 & 4,592 & 250 \\
\hline State Ottshore & 313 & -13 & 36 & 20 & 43 & 0 & $\theta$ & 72 & 293 & 2B7 & 6 \\
\hline Utah & 1,701 & 39 & 193 & 82 & 132 & 17 & 0 & 199 & 1,747 & 1.446 & 301 \\
\hline Vwgrnı & 1,896 & 100 & 46 & 6 & 0 & 0 & 0 & 55 & 1,990 & 1,900 & 0 \\
\hline Weat Wrgnisis & $2,58 \mathrm{~g}$ & 80 & 312 & 92 & 10 & 0 & 74 & 182 & 2,793 & 2722 & 71 \\
\hline Whormang & 12,712 & 79 & 1,267 & 805 & 437 & 0 & 94 & 850 & 13,064 & 12,280 & 624 \\
\hline Foderg Offonore a & 29.518 & 20 & $3 . \mathbf{x}^{2}$ & 2,045 & 1.646 & 736 & 1,767 & 5,095 & 28,419 & $2 \uparrow, 992$ & 7,437 \\
\hline Packe (Cantonla) & 1,299 & 24 & 19 & 47 & 31 & 0 & 0 & 50 & 1,266 & 113 & 1,151 \\
\hline Gulf of Mexhco (Lotessena) ${ }^{a}$ & 21,664 & 243 & 2,030 & 2,346 & 1,388 & 626 & 1,468 & 3,752 & 22,119 & 16,623 & 5,492 \\
\hline Eulf of Mexoco (Taxas) & 0,566 & -4 & 680 & 552 & 227 & 110 & 301 & 1,283 & 6,034 & 5,240 & 794 \\
\hline Arscerlanecors" & $\mathrm{C}_{\mathrm{Bg}}$ & -3 & 11 & 3 & 0 & 0 & 0 & 6 & $\mathbf{8 8}$ & 40 & 28 \\
\hline U.S. Total , . . . . . . . . . . . & 173,476 & 4,200 & 10,034 & 13,767 & 8,189 & 1,491 & 3,209 & $+9,703$ & 170,147 & 144,852 & 30,705 \\
\hline
\end{tabular}

\footnotetext{
Ineluder Federal offshore Alatuanka

Includes Auzona, illarons, Indaana, Marylend, Missoun, Nebraska, Navada, Oregon, South Dakota, and Tennessee

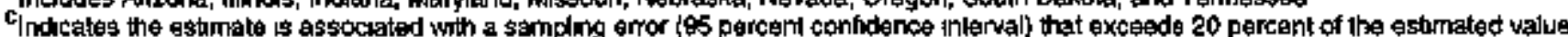

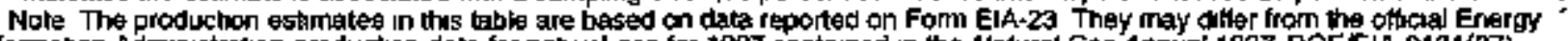

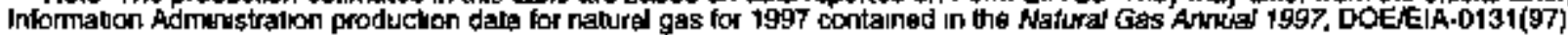

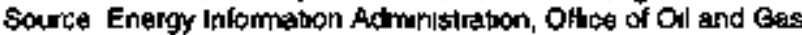


Tabte 10. Nonassociated Natural Gas Proved Reserves, Reserves Changes, and Productlon, Wet After Lease Separation, 1997

(B:llion Cubic Feet at 14.73 psia and $60^{\circ}$ Fahrenhelt)

\begin{tabular}{|c|c|c|c|c|c|c|c|c|c|}
\hline \multirow[b]{2}{*}{ Btabs and substilition } & \multirow[b]{2}{*}{$\begin{array}{l}\text { Publined } \\
\text { Prowed } \\
\text { Feserves } \\
1231 \text { is }\end{array}$} & \multicolumn{7}{|c|}{ Changus in Reserves During̣ 1997} & \multirow[b]{2}{*}{ 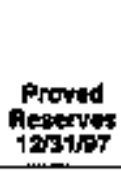 } \\
\hline & & $\begin{array}{c}\text { Aclustment: } \\
\text { (t,.) }\end{array}$ & $\begin{array}{c}\text { Rutidion } \\
\text { mertilests } \\
\text { [+] }\end{array}$ & $\begin{array}{c}\text { Ravision } \\
\text { Dentodists } \\
\text { [t] }\end{array}$ & $\begin{array}{c}\text { Extintidons } \\
{[+]}\end{array}$ & 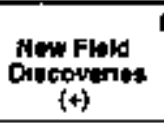 & 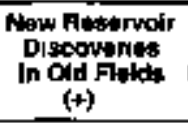 & $\begin{array}{c}\text { Esimatiod } \\
\text { Preduction } \\
(-1)\end{array}$ & \\
\hline Alastion & $\$, \hat{s} \mathbf{1} \boldsymbol{B}$ & -1 & 42 & 93 & 0 & 0 & 0 & स्ग7 & 2,957 \\
\hline Lomer 48 Stater $, \ldots+\ldots, \ldots \ldots$ & $.141,136$ & +491 & 17,745 & 14,321 & 10,063 & 1,307 & 2,280 & 16,905 & $1+0,303$ \\
\hline Alabama & $5,0,82$ & -47 & 346 & 54 & 34 & 0 & 14 & 372 & 4,983 \\
\hline Alkansab & $t, 383$ & 0 & 196 & 94 & 96 & 0 & 15 & 173 & 1,423 \\
\hline Catiomia & 610 & -9 & 41 & 28 & 11 & 0 & $\theta$ & 65 & 570 \\
\hline Coaskal Pognn Onshore & 48 & 9 & 2 & 8 & 0 & 0 & 0 & E & 47 \\
\hline Lus Angoles Beasin Onghore & 0 & 3 & 1 & 0 & 0 & 0 & 0 & 1 & 3 \\
\hline San Joagyin Bassn Onshore & 560 & -21 & 38 & 22 & t1 & 0 & 9 & 57 & 518 \\
\hline Stads Orsthore & 2 & 1 & 0 & 0 & $\theta$ & 0 & 0 & 1 & 2 \\
\hline Colarado & $7, \infty)$ & -474 & 987 & 483 & 99 & 2 & 0 & 533 & $\mathbf{B}, \mathbf{5 2 7}$ \\
\hline Flonda & 0 & 0 & $D$ & 0 & D & 0 & 0 & D & 0 \\
\hline Kanias & $8,0,03$ & -9 & 306 & 497 & 55 & 0 & 4 & 647 & 7,277 \\
\hline Konbuxlyy & 1.022 & 319 & 109 & 2 & 18 & 0 & 18 & 81 & 1.400 \\
\hline Loxicana & 0,038 & -44 & 1,515 & 1,304 & 681 & 5 & 415 & 1,396 & 9.000 \\
\hline North & 2,234 & $9 d$ & 597 & 318 & $13 B$ & 0 & 2 & 368 & 2.869 \\
\hline Boult Onstrore & $5,47 B$ & 22 & 937 & TB3 & 520 & 5 & 321 & $B 62$ & 5,539 \\
\hline Slate Cfishore & 628 & 28 & 81 & $10 \$ 3$ & 25 & 0 & $9 s$ & 13 & 613 \\
\hline Mrchugen & $1.7 \mathrm{rg}$ & 199 & 225 & 74 & 39 & 0 & 0 & 192 & 1,975 \\
\hline Mls5tapspor & 587 & .18 & 68 & $\mathbf{d} \mathbf{8}$ & 36 & 0 & 2 & 85 & 592 \\
\hline Montana & 75 & 35 & 96 & 130 & 21 & 0 & 0 & 51 & 727 \\
\hline Now Haxko & $16,23,2$ & -975 & 2,455 & 1,773 & 680 & 0 & 4 & 1,423 & 15,290 \\
\hline Enat & 1 612 & 9 & 275 & 128 & 175 & 0 & 4 & 253 & $1,60 \mathrm{~d}$ \\
\hline Weal & 14,020 & .694 & 2.180 & 1,645 & 485 & 4 & 0 & 1170 & 13,588 \\
\hline New York & 229 & - $\$ 1$ & 60 & 41 & 0 & 0 & 4 & 18 & 223 \\
\hline North Dakola & 257 & -7 & 23 & 4 & 25 & 0 & 0 & 20 & 274 \\
\hline Ohot & 715 & 63 & 36 & 64 & 5 & 0 & 13 & 49 & 594 \\
\hline Otuehome & 12,929 & 148 & 2340 & 1.2䃇 & 589 & 2 & $\infty$ & 1532 & 13295 \\
\hline Pothosylyantua & 1655 & 59 & 512 & 392 & 49 & 0 & 5 & 123 & 1.789 \\
\hline TExas & 33432 & 81 & 4,0366 & $4, \infty 41$ & 3,719 & 395 & 387 & 4.525 & 33,322 \\
\hline FAC DuAnet 1 & 523 & 205 & 119 & 49 & 149 & 27 & 0 & 104 & $\$ 50$ \\
\hline RBOC Dasiritet 2 Onshore & 1,178 & 51 & 252 & 184 & 317 & 53 & 31 & 201 & 1,497 \\
\hline RAC Distnat 3 Onsthore & 3,612 & -228 & 640 & 430 & 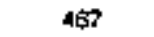 & 205 & 60 & 795 & 363 \\
\hline PAG Distol 4 Dnsther & 7,877 & 47 & 1.193 & 1,207 & 1,449 & 32 & 110 & 1,291 & B.115 \\
\hline ARC Drgknct 5 & 2,098 & 60 & 134 & 6]? & $\theta 9$ & 26 & 155 & 204 & 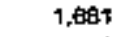 \\
\hline FAC Dsanct e & 5,690 & .10 & 514 & $\sec$ & $6 \operatorname{sos}$ & $B$ & t1 & 58 & 5,616 \\
\hline PRAC Diknct 7E & 378 & 东宗 & 35 & 32 & 1 & 26 & 0 & 45 & 299 \\
\hline PAC Drinkt 7C & 3,371 & -34 & 263 & 294 & 279 & 5 & 3 & 333 & 3,247 \\
\hline RAC Digitel B & $3, \infty 69$ & .119 & $3 \$ \$$ & 155 & 82 & 7 & 10 & 3 & 2.8珀 \\
\hline RRC C16tnct BA & 18 & 35 & 10 & 16 & 0 & 0 & 0 & 7 & 41 \\
\hline RPC Districl 9 & 749 & 50 & 46 & 79 & 119 & 0 & 0 & 100 & 785 \\
\hline RFC Cigmet 10 & 4,592 & -55 & 396 & 344 & 198 & 3 & 4 & 411 & 4.966 \\
\hline State Ottshore & 287 & 22 & 54 & 35 & 17 & 11 & 0 & 76 & 280 \\
\hline Uteh & $1.4 \mathrm{AB}$ & 17 & 248 & 22 & 175 & 0 & 0 & 198 & 1.695 \\
\hline V|rgn: & . 1,930 & .134 & 75 & 16 & 113 & 0 & 0 & $4 \theta$ & 1.023 \\
\hline We:s vigroạ & 2.722 & 1ฆด़ & 139 & 73 & 13 & 0 & 41 & 168 & 2,887 \\
\hline Wroming & 12,260 & 96 & $t .17:$ & 1,404 & 2,145 & 39 & 19 & 854 & 13,471 \\
\hline Fodaral Gilshore & 21,802 & .252 & 2,738 & 2,640 & 1,460 & 944 & 1,239 & 4,393 & 21,098 \\
\hline Pacdic (Calıfomı) & 115 & -53 & 0 & 2 & 0 & 0 & 0 & 2 & 58 \\
\hline Gul of Maroco (Leussane) ${ }^{a}$ & 16,627 & -365 & 2,075 & 1,751 & 1,089 & 797 & 1,056 & 3,297 & 16,241 \\
\hline Guf of hlexocr (Texps) & 5,240 & 168 & 683 & 897 & 391 & 147 & 189 & 1,104 & 4,709 \\
\hline Musodillantough & 40 & 29 & 3 & 0 & 0 & 0 & 0 & 1 & 13 \\
\hline 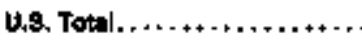 & 144,352 & 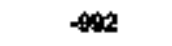 & 17,787 & 14,414 & 10,069 & 1,367 & 2,2609 & 17,112 & 140,339 \\
\hline
\end{tabular}

a Inciudes Foderal ofishore Alabama.

bincludes Anzzona, IIHnots, Indiana, Manyand, Mlssoun, Nebratka, Nevada, Oregon, South Dakota, and Tennessee.

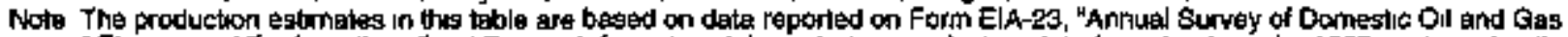
Roservas " They may dhffer fom the oftical Energy Infomkatron Ad mirustraton producton deila lor naturel gas lor 1997 contained in the Nalural Gas Aninuad 1907 , DOEVIA-0131 ( 97 )

Solrte: Energy lialomation Admmisiraton, Othee of OAl and Gas 
Table 11. Associated-Dlssolved Natural Gas Proved Reserves, Reserves Changes, and Production, Wet After Lease Separation, 1997

(Billon Cubic Feet at 14.73 psia and $60^{\circ}$ Fahrenheit)

\begin{tabular}{|c|c|c|c|c|c|c|c|c|c|}
\hline \multirow[b]{2}{*}{ Sisto and Suthdrusion } & \multirow[b]{2}{*}{ 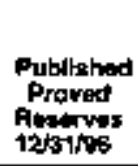 } & \multicolumn{7}{|c|}{ Changes in Raserves During t9g7 } & \multirow[b]{2}{*}{ 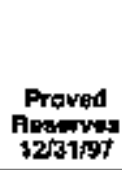 } \\
\hline & & Adjuttrimama & $\begin{array}{c}\text { Raviaion } \\
\text { hucrotesed } \\
(+)\end{array}$ & $\begin{array}{c}\text { Ripwaiten } \\
\text { Deacrasases } \\
\text { (-) }\end{array}$ & $\begin{array}{c}\text { Extanskons } \\
\qquad(+)\end{array}$ & $\begin{array}{c}\text { Naw Fredd } \\
\text { Dracoivents } \\
(+)\end{array}$ & 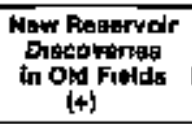 & $\begin{array}{c}\text { Eprimisted } \\
\text { Produotiont } \\
\text { in) }\end{array}$ & \\
\hline Alaska & 6,280 & 106 & 1,792 & 6 & 14 & 0 & 0 & 270 & 7.716 \\
\hline Lowter 48 statea $. ., \ldots, \ldots, \ldots$ & 24,715 & 158 & 3,133 & 3,235 & 1,102 & 1,340 & 187 & 2,752 & $\mathbf{2 4 , 8 8 6}$ \\
\hline Alabam: & 38 & -1 & $\mathbf{5}$ & 2 & 0 & 0 & 0 & 10 & 30 \\
\hline Arkangas & 然 & -19 & to & 7 & 0 & 0 & 0 & 17 & 50 \\
\hline CelMomia & 1,583 & 25 & 336 & 28 & 61 & 0 & 1 & 158 & 1,820 \\
\hline Coastal As & 120 & 10 & 9 & 16 & 16 & 0 & 1 & 11 & 129 \\
\hline Los Angedé Basn Onthere & 112 & 19 & 28 & 6 & 0 & 0 & 0 & 10 & 143 \\
\hline $\operatorname{sen}$ Jarquirn Bastin Onshore & 1,304 & -14 & 296 & 6 & 45 & 0 & 0 & 131 & 1,494 \\
\hline Siele OHfhore & 47 & 10 & 9 & 0 & 0 & 0 & 0 & 6 & 54 \\
\hline Colorads & 1,055 & -59 & 17 & 438 & I5 & 0 & 0 & 57 & $5 \times 3$ \\
\hline Flanda & $1+9$ & -1 & 1 & 0 & 0 & 0 & 0 & 7 & 112 \\
\hline Kansas & 82 & 4 & 14 & 38 & 2 & 0 & 0 & 13 & 51 \\
\hline Kenbucky & 24 & 1 & 3 & 1 & 1 & 0 & 0 & $\mathbf{2}$ & 26 \\
\hline Loulsatara & 1,039 & 73 & 150 & 206 & 104 & 6 & 16 & 165 & 1,018 \\
\hline North & 255 & 7 & 29 & 自7 & 43 & 0 & 0 & 44 & 287 \\
\hline South Onshore & 627 & 15 & 99 & 117 & s5 & 6 & 13 & 93 & 599 \\
\hline Stale Offighore & 157 & 19 & ه & 22 & 白 & 0 & 2 & 28 & 130 \\
\hline Wictingan & 347 & .25 & 34 & 53 & 13 & 0 & 0 & 35 & 291 \\
\hline 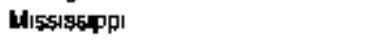 & 47 & o & 8 & a & 12 & 0 & 0 & 8 & 51 \\
\hline Monkane & $\mathbf{5 1}$ & -5 & 4 & 4 & 1 & 0 & 0 & 5 & 42 \\
\hline 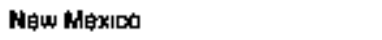 & 1,693 & -3 & 184 & 270 & 44 & 2 & 2 & 232 & 1,420 \\
\hline East & 1,585 & -13 & 170 & 253 & 44 & 2 & 2 & 223 & 1,314 \\
\hline Weal & 108 & 10 & $1 d$ & 17 & 0 & 0 & 0 & 9 & 106 \\
\hline New Yolk & 3 & -1 & D & 1 & 0 & 0 & 0 & 0 & 1 \\
\hline North Dakota & 255 & 5 & 40 & 15 & 3 & 0 & 0 & 31 & $2 \$ 7$ \\
\hline Oheor & sea & +6 & As & 12 & 0 & 0 & 4 & 57 & 391 \\
\hline Oklanoms & 1,023 & -92 & 205 & 97 & 91 & 0 & $g$ & 124 & 1,015 \\
\hline Peninsytuana & 47 & 10 & 10 & 1 & 33 & 0 & 0 & 7 & 92 \\
\hline Texaș & 8, 160 & -370 & 026 & 790 & $\$ 10$ & 3 & 24 & 807 & 7,786 \\
\hline RRC Desinct 1 & 440 & -379 & 7 & 10 & 11 & 0 & a & g & 66 \\
\hline RRC Dembt 2 Oneihere & 252 & 29 & 11 & 37 & 6 & 0 & 0 & 26 & 235 \\
\hline RAC Dsandit 3 Onshore & 1,040 & -149 & 167 & 105 & 49 & 0 & 0 & 143 & 879 \\
\hline 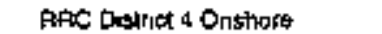 & 248 & 122 & 17 & 28 & 72 & 1 & 0 & E2 & soge \\
\hline RRE Dasinet 5 & 53 & 5 & 32 & 14 & 2 & 0 & 1 & 11 & 68 \\
\hline RAC Desinct 6 & 582 & E & 15 & 42 & 2 & 0 & 0 & 42 & 578 \\
\hline RRC Distued Te & 232 & -47 & 89 & 14 & 53 & 0 & 0 & 33 & 260 \\
\hline RAC D19tncl 7C & 692 & -5 & \$1 & 107 & 自 & 0 & $\theta$ & 81 & 396 \\
\hline PAHC Distnct 8 & 2,801 & .75 & 487 & 281 & 272 & 2 & 14 & 276 & 3,144 \\
\hline RAC OAatnCl BSA & 1,276 & 10 & 4 & 65 & 18 & 0 & 0 & 74 & 1,208 \\
\hline RAC Disthel S & 121 & 37 & 10 & 8 & 10 & 0 & 1 & 24 & 147 \\
\hline RAC Distned 10 & 253 & 3 & 95 & 39 & 7 & 0 & 0 & 28 & 227 \\
\hline Stals Ollsthort & 8 & 1 & 4 & 0 & 0 & 0 & 0 & 1 & 10 \\
\hline Utah & son & .10 & 57 & 15 & 3 & 0 & 0 & 26 & 310 \\
\hline Vinginia & 0 & 478 & 67 & 0 & 0 & 0 & 0 & 22 & 529 \\
\hline Wast Vingonina & 31 & -1 & 3 & 9 & $\theta$ & 0 & a & $\mathbf{5}$ & 59 \\
\hline Wyomung & azd & 54 & 86 & 42 & 15 & 0 & 0 & 87 & 850 \\
\hline Federal Oltshore & 7,437 & 90 & 818 & 1,232 & 194 & 1,349 & 132 & 875 & 7,913 \\
\hline Pactic (Calfomrofe) & 1,151 & .13 & 6 & 512 & 1 & 1 & $\mathbf{0}$ & 38 & 498 \\
\hline 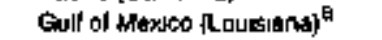 & 5,492 & $18 \mathrm{~g}$ & 695 & $\mathbf{5 0}$ & 186 & 750 & 123 & 697 & 6.167 \\
\hline Culf ol Hexico (Texss) & 794 & -85 & 117 & 70 & 7 & 598 & 9 & 142 & 1.223 \\
\hline Mescallaneous b & $2 B$ & 11 & 20. & 26 & 0 & o & 0 & 2 & 31 \\
\hline It.S. Total. . ................... & 30,795 & 282 & $\mathbf{4 , 9 2 6}$ & 3,241 & 1,118 & 1,350 & 167 & 3,022 & 32,362 \\
\hline
\end{tabular}

Inctudes Federal offshore Alabama

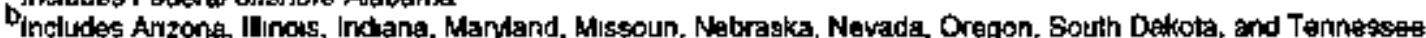

Nole The production estumates in ihss table are based on data reported on Form El|A-23, "Annual Survoy of Domestic Oll and Gas Reserves "They may drffer hrom the othcial Energy Informatorn Adfministratoon productoon data tor naiural gas lop 1997 cortained in the Naturar Clas ANTLei fag7, DOEJEIA-0131 $\{97$

Source Energy information Adrmukstratoon, Ohice of Oil and Cas

Eneroy Infontistion Adminiafration 
Wyoming, and Louisiana accounted for 11,467 billion cubic feet or 84 percent of U.S. NA wet natural gas lotol discoveries in 1997.

\section{Production}

U.S. production of NA wet natural gas increased by 2 percent (267 billion cubic feet) in 1997 (Table 10). Of the five leading producing areas, Texas, the Gulf of Mexico Federal Offshore, and New Mexico increased production, while Louisiana and Oklahoma had slight declines in production. As in 1996, the Gulf of Mexico Federal Offshore and the State of Texas, each with over one-fourth of the U.S. total, were the leading produccrs of NA wet natural gas in 1997.

\section{Associated-Dissolved Natural Gas}

\section{Proved Reserves}

Proved reserves of associated-dissolved (AD) natural gas, wet after lease separation, in the Urited States increased by 5 percent ( 1,587 billion cubic feet) to 32,382 billion cubic feet in 1997 (Table 11). Proved reserves of AD wet natural gas in the lower 48 States decreased by 49 billion cubic feet to 24,666 billion cubjc feet. Those areas of the country with the largest $A D$ wet natural gas reserves were:

- Texas (24 percent)

- Alaska (24 percent)

- Gulf of Mexico Federal Offshore (23 percent)

- California (6 percent)

- New Mexico (4 percent).

These areas logically correspond to the areas of the country with the largest volumes of crude oil reserves.

\section{Production}

U.S. production of $A D$ wet natural gas increased by 3 percent in 1997 (Table 11), and production of $A D$ wet natural gas in the lower 48 States increased by 2 percent (49 billion cubic feet). Those areas of the country with the largest $A D$ wet natural gas production were:

- Gulf of Mexico Federal Offshore (28 percent)

- Texas ( 28 percent)

- Alaska (9 percent)

- New Mexico (8 percent)

- California (5 percent).
Again, these areas logically correspond to the areas of the country with the largest volumes of crude oil production.

\section{Coalbed Methane}

\section{Proved Reserves}

Reserves of coalbed methane increased in 1997 to account for about 7 percent of 1997 dry natural gas reserves (Table 12). Federal tax incentives for new coalbed methane wells expired at the end of 1992 Reserves in coalbed methane fields increased to 11,462 billion cubic feet, 8 percent more than in 1996 . The ELA estimates that the 1997 proved gas reserves of fields identified as having coalbed methane are about double those reported in 1990 (Figure 21). Coalbed methane proved reserves are principally located in New Mexice, Colorado, Alabama, and Virginia. Estimates of proved coalbed methane reserves increased in all these States in 1997 ,

Figure 21. Coalbed Methane Proved Peserves 1989-1997

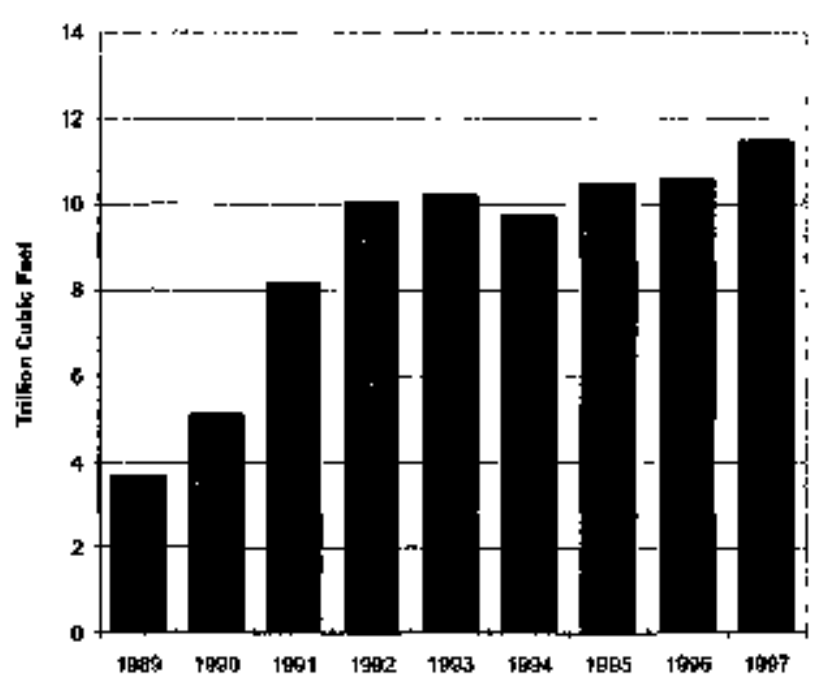

Source: Energy Information Adminislration, Otfice of Oil and Gas.

\section{Production}

Coalbed methane production grew by about 9 percent in 1997 to 1,090 billion cubic feet-about 6 percent of U.S. dry gas production. Most of the 1997 production increase ( 87 billion cubic feet) occurred in the San Juan Basin of Colorado and New Mexico. 
Table 12. U.S. Coalbed Methane Froved Peserves and Production, 1994-1997

(Billion Cubic Feet at 14.73 psia and $60^{\circ}$ Fahrenheit)

\begin{tabular}{|c|c|c|c|c|c|c|c|c|}
\hline Stat: & $\begin{array}{c}\text { 1994 } \\
\text { Raservea }\end{array}$ & $\begin{array}{c}1994 \\
\text { Produetion }\end{array}$ & $\begin{array}{c}1995 \\
\text { Aegaruas }\end{array}$ & $\begin{array}{c}1995 \\
\text { Production }\end{array}$ & $\begin{array}{c}\text { 1996 } \\
\text { Repsinvets }\end{array}$ & $\begin{array}{c}1986 \\
\text { Production }\end{array}$ & Reservas & $\begin{array}{c}1997 \\
\text { Production }\end{array}$ \\
\hline Alabama , . . . . & 978 & 108 & 972 & 109 & 823 & 98 & 1,077 & 111 \\
\hline Colorado....... & 2,913 & 179 & 3,461 & 226 & 3.711 & 274 & 3,890 & 312 \\
\hline New Mexico .... & 4,137 & 530 & 4,299 & 574 & 4,190 & 575 & 4,351 & 597 \\
\hline Oiners $^{a} \ldots . .$. & 1,686 & 34 & 1,767 & 47 & 1,862 & $\$ 6$ & 2,144 & 70 \\
\hline Total $\ldots \ldots \ldots$ & 9,712 & 851 & 10,499 & 956 & 10,566 & 1,003 & 11,462 & 1,090 \\
\hline
\end{tabular}

Anculudes Colahoma, Pannsytrania, Utah, Virginia, West Wieginia, and Wyoming.

Source: Energy Iniormation Adminisiation, Otfice of Oil and Gas.

\section{Areas of Note: Large Discoveries and Reserves Additions}

The following State or area discussions summarize notable activities during the year concerning expected new field reserves, development plans, and possible production rates as extracted from various trade publications and company reports. The citations do not necessarily reflect EIA's concurrence, but are considered important enough to be brought to the reader's attention.

\section{Alaska}

Alaska had a net increase of 1,268 billion cubic feet of dry nattral gas proved reserves in 1997. The largest net gas reserves increase of all States in 1997, it was from adjustments and net revisions.

\section{Gulf of Mexico}

Deep Water: Advarnces in 3-D seismic technology ard previous deepwater successes have encouraged the oil and gas industry to search and drill for hydrocarbons farther offshore. Drilling in the deepwater portions of the Outer Continental Shelf (OCS) of the Gulf of Mexico has greatly increased in the past few years, and is now at an all-time high.

Mensa: Production began in July 1997 from the deepest producing field in the Gulf of Mexico, the Mensa Fjeid in Mississippi Canyon Blocks 686, 687,730 and 731. Shell produces gas from a subsea system of three wells connected to a subsea manifold five miles away, all in 5,300 feet of water. The subsea manifold is tied back to a shallow water platform in West Delta Block 143 via a 63-mile 12-inch diameter flowline. This 68-mile tieback is currently the longest in the world. Shell estimates that the Mensa Field has an ultimate recovery of 720 billion cubic feet of gas and will have peak production rates of 300 million cubic feet of gas per day in the third quarter of 1998. (36)

Marlin: In the Gulf of Mexico Federal Otfshore (Louisiana) in Viosca Knoll Block 915, Amoco is developing the Marlin Field in 3,240 feet of water. Amoco plans in late 1998 to install a tension-leg platform, five producing wells, and full on-deck processing capacity for 40,000 barrels of cil, 250 million cubic feet of gas, and 20,000 barrels of water, per day. The development system will also include two deepwater pipelines to transport the produced oil and gas. Production is expected to begin in 1999. $\{33\}$

Gemini: At the Gemini Field in Mississippi Canyon Block 292, Texaco applied sophisticated 3D imaging to locate and drill into reservoirs that Jie below a 2,900-foot-thick salt sheet which initially defied scientific exploration. Texaco owns $60 \%$ of this subsalt gas and condensate discovery. Texaco expects the Gemini Field to reach a peak rate of about 180 million cubic feet per day by mid-1999.\{37\}

\section{Texas}

Although the State of Texas had a net decline in dry natural gas proved reserves in 1997, the second largest volume of new discoveries in 1997 was nevertheless made there.

South Texas: Operators remain active in the Lobo Trend in the lower Rio Grande Valley of south Texas (RRC District 4). The trend occurs ptimarily in Webb and Zapata counties and contains the four producing horizons, Wilcox, Expanded Wilcox, Frio, and Lobo. Unlike some other parts of the country, one or two fields do not dominate the area. RRC District 4 
increased its dry natural gas reserves by 330 billion cubic feet in 1997. Operators in RRC District 4 reported more extensions ( 37 percent of the State total) than any other district in Texas. This district accounts for 2.1 percent of all of the reserves of dry natural gas in tre State and leads the State in gas production (26 percent of the State total). RRC District 4's dry gas production increased 2 percent in 1997.

Similarly, RRC District 2 had an increase in dry natural gas reserves of 312 billion cubic feet in 1997.mostly from exiensions of existing gas fields. RRC District 2's dry gass production increased 13 percent in 1997.

\section{Wyoming}

In 1997, Burlington Resources acquired Louviana Land and Exploration Company, which consolidated Burlington Resources' holdings in the Madden Field in Fremont County, Wyoming. On March 19, Louisiana Land and Exploratton Company (LL\&E) announced a major extension to the reserves of the Madden Fielc. Open hole $\log$ analysis of the new Big Hom 4-36 well indicated a gross gas column of 260 feet in the Madison formation with no water and excellent reservoir quality. As a result, LL\&E increased its estimate of proved and probable, recoverable natural gas reserves in the Madison reservoir to mose than one trillion cubi: feet. The Big Hom 4-36 well, with a total depth of 24,600 feet, was drilled to a location in the gas-bearing Madison formation 1,000 feet downdip from the crest of a subsurface structure which has a minimum areal extent of 23 square miles.

The Big Horn 4-36 well was drilled as a step-out frorr the Big Horn 1-5 and the Big Horn 2-3 wells. Theses: wells are currently completed in the Madisork formation and are together producing about 52 million cubic feet a day into the Lost Cabin Gas Plant (the current inlet capacity of the plant). Each well is capable of sustained production levels in excess of $\mathbf{3 0}$ million cubic feet a day. Carbon dioxide and hydrogen sulfids! ane removed prior to the sale of the gas and residual gas: sales average approximately 34 million cubic feet a day. $\{38\}$

\section{Areas of Note: Large Reserves Declines}

The following areas had large declines in dry natural gas proved reserves due to downward revisions or unreplaced production.

\section{New Mexico}

This State's proved dry natural gas reserves decreased by 6 percent ( 971 billion cubic feet). Two fields (Basin and Blanco) accounted for about one-fourth of the production and about one half of the total reserves of gas in New Mexico.

\section{Colorado}

This State's proved dry natural gas reserves decreased by 11 percent ( 882 billion cubic feet). However, the stage is set for a future increase in reserves due to a strategic acquisition by operator HS Resources. Near the end of 1997, HS Regources acquired Amoco's Denver- Julesburg Basin properties (in and surrounding Colorado's large Wattenberg field), capping an effort that spanned many years. For HS Resources, the compelling reason for the acquisition was the extraordinary abundance of reserves that could be unlocked by combining the Amoco properties with its own. For example, in many instances HS Resources owns the right to produce from formations which are "behind pipe" in an Amoco producing well. Bringing these reserves into production by recompleting an existing well generates substantially better economics than can be achieved by drilling a new well. In other instances, Amoco owned the right to produce from formations located deeper than horizons from which HS Resources was already prodtucing and deepening an existing well is substantially more economic than drilling a new well. Without these efficiencies, many potential proved reserves were economically "stranded" because drilling a new well could not be justified. The re-aggregation of reserves and value created by the Amoco acquisition gives HS Resources a substantial inventory of projects with virtually no risk and exceptional rates of return. $\{39\}$ 


\section{Kansas}

This State's proved dry natural gas reserves decreased by 9 percent ( 705 billion cubic feet).

\section{Reserves in Nonproducing Reservoirs}

Nonproducing proved natural gas reserves (wet after lease separation) of 33,124 billion cubic feet were reported in 1997 (Table 13). This was 75 billion cubic feet higher than in 1996. About 37 percent of the reserves in nonproducing reservoirs are located in the Gulf of Mexico Federal Offshore area. Much of the new deepwater reserves are in the nonproducing category. Wells or reservoirs are nonproducing due to any of several operational teasons. These include:

- wajting for well workovers

- waiting for additional development or replacement wells to be drilled

- production or pipeline facilities not yet installed

- awaiting depletion of other zones or reservoirs before recompletion in reservoirs not currently open to production (called "behind pipe" reserves). 
Table 13. Reported Reserves of Natural Gas, Wet After Lease Separation, in Nonproducing Reservoirs, $1997^{3}$

(Billion Cubic Feet at 14.73 pida and $60^{\circ}$ Fahrenheil)

\begin{tabular}{|c|c|c|c|}
\hline State end Subdivision & Nonassacleted Gas & $\begin{array}{l}\text { Associaled- } \\
\text { Dissolved Gas }\end{array}$ & Total \\
\hline Alaska $\ldots \ldots \ldots \ldots \ldots \ldots$ & 232 & 82 & 314 \\
\hline Lower \&s states . . . . . . . . . . . . . . & 27,294 & 5,549 & 32,043 \\
\hline Allabama $\ldots \ldots \ldots \ldots \ldots \ldots$ & 359 & 4 & 363 \\
\hline Arkansas $\ldots \ldots \ldots \ldots \ldots \ldots$ & 136 & 10 & 146 \\
\hline$\ldots \ldots \ldots$ & 103 & 130 & 233 \\
\hline Coastal Region Onshore . . . . . . . & 2 & 7 & 9 \\
\hline Los Angeles Basin Onshore. . . . . . . . & 0 & 34 & 34 \\
\hline San Joqquin Basin Onshore. . . . . . & to1 & a9 & 190 \\
\hline State Otfshore $\ldots \ldots \ldots \ldots$ & 0 & 1 & 1 \\
\hline Coltrado . . . . . . . . . & 600 & 74 & 674 \\
\hline Flonida $\ldots \ldots \ldots$ & 0 & 0 & 0 \\
\hline Kansas $\ldots \ldots \ldots \ldots$ & 144 & 2 & 146 \\
\hline$\ldots \ldots$ & 137 & 0 & 137 \\
\hline$\ldots \ldots \ldots$ & 2,946 & 362 & 3,308 \\
\hline . & 896 & 86 & 982 \\
\hline South Onshore, $\ldots \ldots \ldots \ldots$ & 1,794 & 225 & 2,019 \\
\hline South state offshore . . . . . . . . & 256 & 51 & 307 \\
\hline Michigan $\ldots \ldots \ldots \ldots$, & 77 & 10 & 87 \\
\hline Mississlppi . . . . . . . . . . . & 56 & 14 & 70 \\
\hline Montana . . . . . . . . . . . . . & 41 & 2 & 43 \\
\hline New Mexico. . , , . . . . . . . . & 1,700 & 66 & 1,766 \\
\hline East $\ldots \ldots \ldots \ldots \ldots$ & 236 & 63 & 299 \\
\hline$\ldots \ldots \ldots$ & 1,464 & 3 & 1,467 \\
\hline New York, $\ldots \ldots \ldots \ldots \ldots$ & 2 & 0 & 2 \\
\hline North Dakata , . . . . & 109 & 10 & $\uparrow 19$ \\
\hline Ohio. . . . . . . . . . . & 6 & 0 & 6 \\
\hline Oklahoma. . & 1,219 & 107 & 1,326 \\
\hline Pennsylvartia & 24 & 0 & 24 \\
\hline Texas. . . . . . . . . . . & $\$ 5,734$ & 712 & 6,446 \\
\hline RAC District $1 \ldots \ldots \ldots$ & 244 & 2 & 246 \\
\hline RRC District 2 Onshore . . . . . . . . & 339 & 65 & 404 \\
\hline FAC District 3 Onshors $\ldots \ldots \ldots$ & 730 & 39 & 768 \\
\hline RRC District 4 Onshore & 2,024 & 80 & 2,104 \\
\hline RRC District $5 \ldots \ldots \ldots \ldots$ & 323 & 3 & 326 \\
\hline ARC District $6 \ldots \ldots \ldots \ldots$ & 862 & 128 & 990 \\
\hline RRC District $78 \ldots \ldots \ldots$ & 15 & 20 & 35 \\
\hline RAC District $70 \ldots \ldots$ & 255 & 30 & 285 \\
\hline RRC District $8 \ldots \ldots \ldots \ldots$ & 398 & 281 & 659 \\
\hline RRC District BA & 0 & 74 & 74 \\
\hline RAC Distric1 $9 \ldots \ldots \ldots$ & 39 & 2 & 40 \\
\hline RAC District 10 . & 444 & $\mathbf{8}$ & 452 \\
\hline State Offshore . . . . . . . . . . & 62 & 0 & 62 \\
\hline Utah. . . . . . . . . . & 347 & 94 & 441 \\
\hline Virginia . . . . . . . . . . . & 839 & 0 & $\mathbf{8 3 6}$ \\
\hline West Virginia & 375 & 4 & 379 \\
\hline Wroming & 3,709 & 94 & 3,803 \\
\hline Federal Otrshore $\ldots \ldots \ldots \ldots \ldots$ & $\$ 3,629$ & $\mathbf{3 , 8 5 6}$ & 12,485 \\
\hline Pacific (Califomia) . . . . . . . . . . . & 47 & 19 & 66 \\
\hline Gull of Mexico (Louisiana) $\ldots \ldots \ldots$ & 6,809 & 2,980 & 9,769 \\
\hline Gull of Mexico (Texas) $\ldots \ldots \ldots \ldots$ & 1,773 & 877 & 2,650 \\
\hline Miscellaneouss $^{\mathrm{c}} \ldots \ldots \ldots \ldots \ldots$ & 1 & 2 & \\
\hline 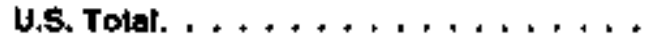 & $2 i, 526$ & 5,681 & 30,157 \\
\hline
\end{tabular}

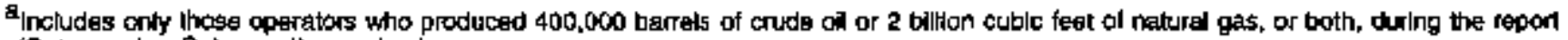
year (Category I or Category II operators).

binchudes Fonderal OHtshore Alabama.

Gncludes Arkorka, Illincis, Indiana, Mtaryland, Missourl, Nebraskg, Nevada, Oregon, South Dakota, and Ternessee.

Sourco: Form ElA-23, "Annual Survey ol Domestic Oll and Gas Regerves," 1997. 


\section{Natural Gas Liquids Statistics}

\section{Natural Gas Liquids}

\section{Proved Reserves}

U.S. natural gas liquids proved reserves increased 2 percent to 7,973 million barrels in 1997 (Table 14). This was the thind annual increase in a row. The 150 million barrel increase was predominantly in Alaska, which nearly doubled it natural gas liquids reserves level in 1997. Overall, reserve additions replaced 117 percent of 1997 natural gas liquids production.

The reserves of six areas account for 83 percent of the Nation's natural gas liquids proved reserves.

$\begin{array}{lc}\text { Arsa } & \text { Percent of } \\ \text { Texas } & \text { NGL Feserves } \\ \text { Gutf of Mexice Federal Otfshore } & 34 \\ \text { New Mexico } & 11 \\ \text { Utah-Wyoming } & 11 \\ \text { Oklahoma } & 10 \\ \text { Alaska } & 9 \\ \text { Area Tota: } & 8 \\ \end{array}$

The volumes of natural gas liquids proved reserves and production shown in Table 14 are the sum of the nafural gas plant liquid volumes listed in Table 15 and the lease condensate volumes listed in Table 16.

\section{Discoveries}

Total discoveries of natural gas liquids reserves increased by 18 percent in 1997 to 739 million barrels, the second highest level ever recorded by ELA Areas with the largest total discoveries were:

- rexas (44 percent)

- Gulf of Mexico Federal Offshore (22 percent)

- Utah and Wyoming (13 percent)

- Louisiana (7 percent)

- New Mexico (6 percent)

- Oklahoma (5 percent).

New fied discoteries in 1997 (114 million barrels) were 75 percent higher than in 1996. Areas with the largest net field discoveries were the Gulf of Mexico Federal Offshore (54 percent of 1997 new field discoveries) and Texas ( 46 percent).

New reserwoir discoueries in old fields ( 90 million barrels) were 17 percent less than what they were in 1996. Areas with the largest new reservoir discoveries in old fields were the Gulf of Mexico Federal Offshore (53 percent of 1997 new reservoir discoveries in old fields), Louisiana (21 percent), and Texas (16 percent).

Exiensions were 535 million barrels, an increase of 19 percent over 1996. Areas with the largest extensions were Texas (48 percent of 1997 extensions), Utah-Wyoming (18 percent), and the Gulf of Mexico Federal Offshore (10 percent).

\section{Production}

Natural gas liquids production was an estinated $\mathbf{8 6 4}$ million barrels in 1997. Alaska production increased 6 percent to 35 million barrels in 1997 , while fower 48 States production incteased 1 percent to 829 million barrels in 1997.

Seven areas accounted for about 90 percent of the Nation's nahurał gas liquids production.

- Texas (37 percent)

- Gulf ol Mexico Federal Offshore (16 percent)

- New Mexico (9 percent)

- OkJahoma (9 percent)

- Louisiana (8 percent)

- Utak-Wyoming (7 percent)

- Alaska (4 percent).

\section{Natural Gas Plant Liquids}

\section{Proved Reserves}

Natural gas plant liquid\$s proved reserves increased 2 percent in 1997 to 6,632 million barrels (Table 15). Six areas accounted for about $\mathbf{8 3}$ percent of the Nation's natural gas plant liquids proved reserves: 
Table 14. Natural Gas Llquids Proved Reserves, Reserves Changes, and Production, 1997 (Mitlion Barrels of 42 U.S. Gallons)

\begin{tabular}{|c|c|c|c|c|c|c|c|c|c|}
\hline \multirow[b]{2}{*}{ State and Supdruston } & \multirow[b]{2}{*}{ 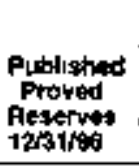 } & \multicolumn{7}{|c|}{ Chancios in Raserves During 1997} & \multirow[b]{2}{*}{ 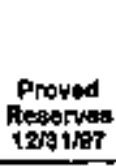 } \\
\hline & & $\begin{array}{l}\text { Adyustments } \\
(4,-)\end{array}$ & 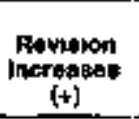 & 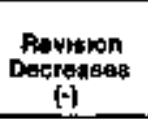 & $\begin{array}{c}\text { Extengasns } \\
{[+]}\end{array}$ & $\begin{array}{c}\text { Now Fuld } \\
\text { Disconterita } \\
(t)\end{array}$ & 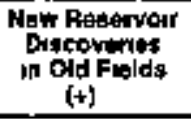 & $\begin{array}{c}\text { Eshimatiod } \\
\text { Producilon } \\
\{x\end{array}$ & \\
\hline Alaşka & $\$ 37$ & 93 & 234 & 0 & 2 & 0 & 0 & 35 & 631 \\
\hline Lomer as siated , $\ldots++, \ldots+\cdots+$ & 7,486 & -107 & \$65 & $\$ 10$ & $\mathbf{5 3 3}$ & 114 & 80 & 129 & 7,342 \\
\hline Ababama & 119 & .13 & 3 & 6 & 0 & 0 & 0 & 10 & 93 \\
\hline Arkangas & $\$$ & 2 & 2 & 0 & 0 & 0 & 0 & 1 & 7 \\
\hline Calformia & 92 & .5 & 15 & 2 & $\mathbf{3}$ & 0 & 0 & 安 & 95 \\
\hline Coasdat Reglon Dnshor日 & $\theta$ & 0 & 1 & 1 & 1 & 0 & 0 & 1 & 9 \\
\hline Los Angelas Basin Onshor to & 3 & 0 & 1 & 0 & Q & 0 & 0 & 0 & 4 \\
\hline San Jompun Oasn Onshore & 80 & .5 & 13 & 1 & 2 & 0 & 0 & $?$ & 82 \\
\hline State Qlfshors & 0 & 0 & 0 & 0 & 0 & 0 & 0 & 0 & 0 \\
\hline Colorado & 287 & 25 & $\$ 4$ & 64 & 5 & 0 & 0 & 23 & $20 \mathbf{4}$ \\
\hline Flonda & 22 & -4 & 0 & 0 & 0 & 0 & 0 & 1 & 17 \\
\hline Kartsas & 338 & 39 & 16 & 21 & 2 & 0 & 0 & 25 & 271 \\
\hline Kentucky & 46 & 1 & 4 & 0 & 1 & 0 & 1 & 3 & 49 \\
\hline Lounsiand & 643 & .109 & 82 & 63 & 35 & $\theta$ & 19 & 70 & 437 \\
\hline Norith & BS & B & 18 & 11 & 6 & 0 & ถ & 12 & 60 \\
\hline Soun Onshare & 411 & 81 & 57 & 45 & 28 & 0 & 16 & 53 & 333 \\
\hline State Ofishore & 47 & -22 & 7 & 7 & 1 & 0 & 3 & $\mathbf{s}$ & 34 \\
\hline MLchigan & 59 & 1 & 白 & 5 & 1 & $\mathbf{a}$ & 0 & 5 & 坫 \\
\hline Messaesapp & 7 & 0 & 0 & 1 & 1 & 0 & 0 & 1 & $\mathbf{s}$ \\
\hline Montana & 7 & 2 & 1 & 1 & 0 & 0 & 0 & 0 & 5 \\
\hline New Mecoco & 1,058 & -156 & 132 & 134 & 47 & 0 & 1 & 80 & 889 \\
\hline Easl & 299 & -11 & $\$ 2$ & 36 & 21 & 0 & 1 & 13 & 273 \\
\hline Yesl & 700 & $1 ₫ 5$ & 90 & 98 & $2 \theta$ & 0 & 0 & 37 & 896 \\
\hline North Dekota & 48 & 2 & 5 & 2 & 2 & 0 & 0 & $\mathbf{d}$ & 47 \\
\hline Dklahoma & 584 & -21 & 134 & 68 & 91 & 0 & 4 & 79 & 685 \\
\hline Texas & 2006 & 81 & 398 & 341 & 255 & 53 & 14 & $3 \times 7$ & 2.687 \\
\hline RAC DIEInç| 1 & 46 & 0 & 7 & 3 & 9 & $\mathbf{1}$ & 吊 & B & \$4 \\
\hline ARC Dis|nis| 2 Onshor & 99 & .15 & 14 & 12 & 18 & 2 & 1 & 12 & $\mathbf{s 7}$ \\
\hline ARC Distnks 3 Chtrora & $\mathbf{2 8 9}$ & 32 & 47 & 37 & 24 & 45 & 3 & 53 & 286 \\
\hline PRC DIEInal 4 Onahore & 3 & 15 & 75 & 105 & as & $\mathbf{\tau}$ & 4 & $\$ 4$ & 347 \\
\hline FAC Distind 5 & 54 & 7 & 3 & 15 & 1 & 0 & 3 & 4 & 35 \\
\hline RRMG Disincl 6 & 290 & -19 & 24 & 34 & 25 & 0 & 0 & 27 & 260 \\
\hline RRAC Disinct TB & 的 & .15 & 14 & $\mathbf{5}$ & $\mathbf{5}$ & 3 & 0 & B & 59 \\
\hline PRE Distrel TC & 303 & 39 & 33 & 30 & 23 & 0 & 1 & 36 & 327 \\
\hline RRXC Disincl : & 429 & 17 & 63 & 33 & 29 & 1 & 2 & 49 & 459 \\
\hline RFC Disinci Bn & 262 & 50 & 12 & 19 & 4 & 0 & 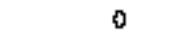 & 19 & 290 \\
\hline RRC Dibruct 9 & 119 & -18 & 6 & 9 & 13 & 0 & 0 & 13 & $\theta$ \\
\hline RRC DISInc| 10 & $3: 32$ & 54 & 37 & 33 & 18 & 0 & 0 & 36 & 382 \\
\hline Shale Olfithore & 1 & 1 & 1 & 0 & 0 & 0 & 0 & 0 & 3 \\
\hline Ulah and womng & 727 & 4 & 73 & 77 & 97 & 0 & 2 & 57 & 761 \\
\hline West Wirgmia & G1 & 11 & 4 & 2 & 0 & 0 & 1 & 4 & 71 \\
\hline Federal Ofilshorg & 776 & 12白 & 196 & 121 & 53 & 61 & 48 & 141 & 920 \\
\hline Pancdic (C)anloma) & 20 & 3 & 0 & 11 & 0 & 0 & 0 & 1 & 14 \\
\hline Gulf of Mexico (Loukspana) ${ }^{\text {t }}$ & 61 & 125 & $10 \mathrm{~s}$ & 89 & 45 & 56 & 45 & 123 & 705 \\
\hline Gulf of Męx|co [Texas] & 132 & 0 & 11 & $2 T$ & 当 & $\mathbf{s}$ & 3 & 17 & 127 \\
\hline Hesodlaneous ${ }^{\circ}$ & 7 & 1 & 2 & 1 & 0 & 0 & 0 & 0 & 9 \\
\hline US, Tolven, ......, , ... & 7,829 & -14 & $t, 199$ & 910 & 535 & 114 & $\mathbf{9 0}$ & 984 & 7,973 \\
\hline
\end{tabular}

andideder Federal offabore Alabairia

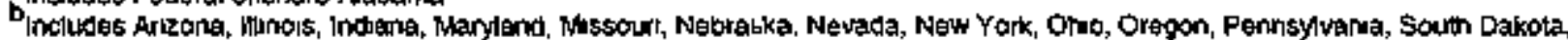

Tennesstese, and Virginia

Note The productocn estimales in this table are based on deta reporled an Form ElA.23, "Arnuel Sunvey of Domegbc Onl and Gas

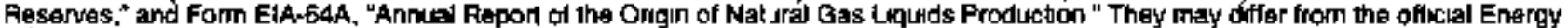
Informaton Admonistration produchon dala for natured gas and nahural gas hquids for 1997 contaned wn the publicabors Petroletum Supply

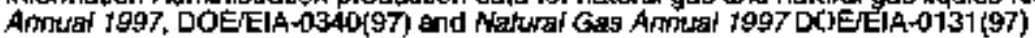

Source Energy Information Admintsiraton, Office of Oll and Gas 
Table 15. Natural Gas Plant Liquids Proved Peserves and Production, 1997 (Million Barrels of 42 U.S. Gallons)

\begin{tabular}{|c|c|c|c|c|c|}
\hline State and Subtilvigion & $\begin{array}{c}1997 \\
\text { Apserves } \\
\end{array}$ & $\begin{array}{c}1997 \\
\text { Prodivolien }\end{array}$ & State and subdivision & $\begin{array}{c}1997 \\
\text { Resserves }\end{array}$ & $\begin{array}{c}1997 \\
\text { Production }\end{array}$ \\
\hline Alaska $\ldots \ldots \ldots \ldots \ldots \ldots \ldots$ & 631 & 35 & North Dakota $\ldots \ldots \ldots \ldots \ldots$ & 40 & 4 \\
\hline Lower 46 Slates. $\ldots \ldots \ldots \ldots \ldots$ & 6,001 & 655 & Oklahoma . . . . . . . . . . . . & 610 & 71 \\
\hline Alabama $\ldots \ldots \ldots \ldots \ldots \ldots \ldots$ & 34 & 4 & Texas $\ldots \ldots \ldots \ldots \ldots \ldots$ & 2,376 & 275 \\
\hline Arkansas. . . . . . . . . . . . . . & 3 & 0 & RFC District 1. $\ldots \ldots \ldots \ldots$ & 46 & 5 \\
\hline Calfomia $\ldots \ldots \ldots \ldots \ldots \ldots$ & 92 & 8 & RRC Disirict 2 Onshore . . . . . & 75 & 10 \\
\hline Coastal Region Onșhore . . . . . & $\mathbf{9}$ & 1 & ARC District 3 Onshore . . . . . & 170 & 36 \\
\hline Los Angeles Basin Onshore. . . & 4 & 0 & RAC District 4 Onsthore . . . . . . & 261 & 42 \\
\hline San Joaquin Basin Onshore. . . & 79 & 7 & RRC District $5 \ldots \ldots \ldots \ldots \ldots$ & 31 & 4 \\
\hline State Offshore............ & 0 & 0 & PAC District $6 . . \ldots \ldots \ldots \ldots$ & 223 & 22 \\
\hline 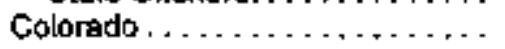 & 244 & 17 & RRC District 7B. . . . . . . . & 57 & 8 \\
\hline Florida $\ldots \ldots \ldots \ldots \ldots \ldots \ldots$ & 17 & 1 & RRC Disirict $7 \mathrm{C} \ldots \ldots \ldots \ldots$ & 309 & 34 \\
\hline Kanses $\ldots \ldots \ldots \ldots \ldots \ldots$ & 263 & 24 & FRC District $8 \ldots \ldots \ldots \ldots \ldots$ & 447 & $4 \overrightarrow{7}$ \\
\hline Kentucky . . . . . . . . & 47 & 3 & RAC District $8 \mathrm{~A} \ldots \ldots \ldots \ldots$ & 290 & 19 \\
\hline Louisiana. . . . . . . . . . & 261 & 41 & RRC District $9 \ldots \ldots \ldots \ldots \ldots$ & 96 & 13 \\
\hline North $\ldots \ldots \ldots \ldots \ldots \ldots$ & 50 & $\begin{array}{r}41 \\
7\end{array}$ & RPC District $10 \ldots \ldots \ldots \ldots$ & 372 & $\$ 5$ \\
\hline South Onshore $\ldots \ldots \ldots \ldots \ldots$ & 50 & 7 & State Offshore. . . . . . . . . . . & 1 & 0 \\
\hline $\begin{array}{l}\text { South Onshore } \ldots \ldots \ldots \ldots \ldots \\
\text { State Otfshore. . . . . . . . . . }\end{array}$ & 199 & 31 & Utah and Wyoming . . . . . . . . . & 680 & 49 \\
\hline $\begin{array}{l}\text { State Qinsnore. } \ldots \ldots \ldots \ldots \ldots \\
\text { Michigan }, \ldots \ldots \ldots \ldots \ldots \ldots\end{array}$ & 36 & 3 & West Virginia. . . . . . . . . . . & 70 & 4 \\
\hline $\begin{array}{l}\text { Michigan }, \ldots \ldots \ldots \ldots \ldots \ldots \ldots \ldots \\
\text { Missisgioni. }\end{array}$ & 12 & 4 & Federal Offshore ${ }^{a} \ldots \ldots \ldots \ldots$ & 389 & 71 \\
\hline Mississippi. , . . . . . . . . . . . . & 45 & 0 & Pacitic (Calitornia) . . . . . . . & 10 & 1 \\
\hline Moniarna $\ldots \ldots \ldots \ldots \ldots \ldots$ & $\mathbf{G}$ & 0 & Gult of Mexico (Loulsiana) ${ }^{\mathbf{a}}$... & 352 & 64 \\
\hline New Mexico $\ldots \ldots \ldots \ldots \ldots \ldots$ & 814 & 75 & Gull of Mexico (Texas\} . . . . . . . & 27 & 6 \\
\hline Eest $\ldots \ldots \ldots \ldots \ldots \ldots \ldots$ & 253 & 40 & Mlscellaneous $\ldots \ldots \ldots \ldots \ldots$ & $\theta$ & 0 \\
\hline 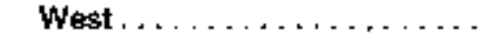 & 561 & 36 & U,S. Total , . . . . . . . . . . . . & 6,632 & 690 \\
\hline
\end{tabular}

\footnotetext{
Includes Federal OHahore Alabama.

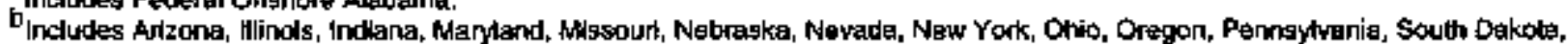
Ternessefe, and Virginia.

Note: The oroduction estimales in $\forall$ ts table are based on data reporied on Form ElA-23, "Annual Survey of Domestic Di and Gas

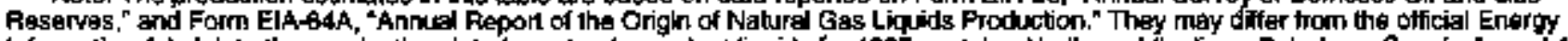

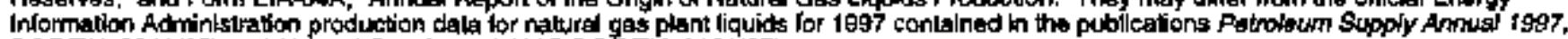
DOEEE A-0340(97) and Natural Gas Annuad 1997, DOE/ELA-0131(97).

Source: Enargy Intormation Adrninistretion, Offles of Oil and Gas.
} 
Table 16. Lease Condensate Proved Resarves and Production, 1997 (Million Bartels of 42 U.S. Gallons)

\begin{tabular}{|c|c|c|c|c|c|}
\hline State and Subdivision & $\begin{array}{c}1997 \\
\text { Reserves: }\end{array}$ & $\begin{array}{c}1997 \\
\text { Producilan }\end{array}$ & Stale and Subdlylsian. & $\begin{array}{c}1997 \\
\text { Rederves }\end{array}$ & $\begin{array}{c}1997 \\
\text { Production }\end{array}$ \\
\hline Alaska . . . . . . . . . . . . . & 0 & 0 & North Dakota . . . . . . . . . . . . & 7 & 0 \\
\hline Lower 48 Stales. . . . . . . . . . . & 1,341 & 174 & Oddahoma $\ldots . . \ldots \ldots \ldots \ldots$ & 76 & 8 \\
\hline Alabama $\ldots \ldots \ldots \ldots \ldots \ldots$ & 59 & 6 & Texas $\ldots . . . . . . . . .$. & 311 & $\$ 2$ \\
\hline Arkankas. . . . . . . . . . . . & 4 & 1 & RRC District $1 \ldots \ldots \ldots \ldots$ & 8 & 1 \\
\hline Califomla. . . . . . . . . . . . . & 3 & 0 & RRC District 2 Onshore . . . . . & 12 & 2 \\
\hline Coastal Region Onshore . . . . . & 0 & 0 & RRC District 3 Onshopre . . . . . & 116 & 17 \\
\hline Los Angeles Basin Onshore. . . & 0 & 0 & RRC District 4 Onshore $\ldots \ldots$ & B6 & 12 \\
\hline San Joaquin Besin Onshore. . . & 3 & 0 & RRC District $5 . \ldots \ldots \ldots \ldots$ & 4 & 0 \\
\hline State Otfshore. . . . . . . . . . & 0 & 0 & RRC District $6 . \ldots \ldots \ldots \ldots$ & 39 & 5 \\
\hline 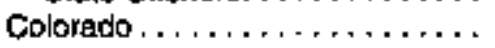 & 20 & 2 & RRC District 7B,$\ldots \ldots \ldots \ldots$ & 2 & 0 \\
\hline Florida $\ldots \ldots \ldots \ldots \ldots \ldots \ldots$ & 0 & $\overline{0}$ & RRC District 7C . . . . . . . & 18 & 2 \\
\hline Kansas $\ldots \ldots \ldots \ldots \ldots \ldots \ldots$ & 8 & 1 & RRC District $8 \ldots \ldots \ldots \ldots \ldots$ & 12 & 2 \\
\hline Kentucky............ & 1 & 0 & RRC District BA . . . . . . & 0 & 0 \\
\hline Louisiana. $\ldots \ldots \ldots \ldots \ldots \ldots$ & 17 & 0 & RAC Diștrict $9 . \ldots \ldots \ldots \ldots$ & 2 & 0 \\
\hline $\begin{array}{r}\text { Loussiana, } \ldots \ldots \ldots \ldots \ldots \ldots \\
\text { North. } \ldots \ldots \ldots \ldots \ldots \ldots \ldots\end{array}$ & 176 & 29 & RRC District 10. . . . . . . . & 10 & 1 \\
\hline $\begin{array}{l}\text { North } \ldots \ldots \ldots \ldots \ldots \ldots \ldots \\
\text { South Gishore } \ldots \ldots \ldots \ldots \ldots \ldots\end{array}$ & 30 & $\mathbf{5}$ & State Offshore. . . . . . . . . & 2 & 0 \\
\hline $\begin{array}{l}\text { South Dishore } \ldots \ldots \ldots \ldots \ldots \\
\text { State Ofishore. . . . . . . . . . . }\end{array}$ & 134 & 22 & Utah and Wyoming $\ldots \ldots \ldots \ldots$ & 81 & 8 \\
\hline $\begin{array}{l}\text { State olishore. } \ldots \ldots \ldots \ldots \ldots \\
\text { Michigan } \ldots \ldots \ldots \ldots \ldots \ldots \ldots\end{array}$ & 12 & 2 & West Virglnia . . . . . . . . . . . & 1 & 0 \\
\hline 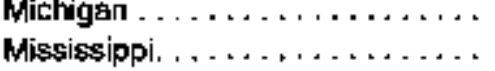 & 5 & 1 & Federal Offshore ${ }^{a} \ldots \ldots \ldots \ldots$ & 631 & 70 \\
\hline $\begin{array}{l}\text { Mississippi. . . . . . . . . . . . . . . } \\
\text { Montena . . . . . . . . . . . . . . }\end{array}$ & 4 & 1 & Pacitic (Calltornia). . . . . . . . . & 4 & 0 \\
\hline $\begin{array}{l}\text { Montena } \ldots \ldots \ldots \ldots \ldots \ldots \ldots \\
\text { New Mexico } \ldots \ldots \ldots \ldots \ldots \ldots\end{array}$ & D & 0 & Gulf of Mexico (Louvisiana) ${ }^{a} \ldots$ & 433 & 59 \\
\hline $\begin{array}{l}\text { New Mexico } \ldots \ldots \ldots \ldots \ldots \\
\text { East } \ldots \ldots \ldots\end{array}$ & 55 & $\mathbf{5}$ & Gulf of Mexiç (Texas) . . . . . . & 94 & 11 \\
\hline East $\ldots \ldots \ldots \ldots \ldots \ldots \ldots$ & 20 & 3 & Miscellaneous ${ }^{\circ} \ldots \ldots \ldots \ldots . . . .$. & 0 & 0 \\
\hline West . . . . . . . . . . . . & 35 & 2 & U.S. Total . . . . . . . . . . . . & $1,34 t$ & 174 \\
\hline
\end{tabular}

乌nchudes Federal Ottghore Alebarma.

Inchudes Arizona, Ifinois, Indanz, Marytand, Mlssouri, Nebraska, Nevgda, New York, Ohio, Oregon. Penrnsylvania, South Dakotg, Tennesses, and Virginia.

Note: The equmates in this table are based on date fepprted on Form ElA-23, "Annual Survey of Domestic Oil and Gas Reserves" 1997.

Sourcet: Energy Information Admbișirabon, Office of OAl and Clas. 
Parbent of

Arẹa v.S. Gas Plant Liquids

Texas 36

New Mexico

12

Utah-Wyoming

10

Alaska

Oklahoma

Gulf of Mexico Federal Orfshore

Area Total

\section{Production}

Natural gas plank liquids production increased only slightly in 1997-from 688 million barrels in 1996 to 690 million barrels of production (Table 15). The top six areas for proved reserves of nakural gas plant liquids accounted for about 83 percent of the Nation's natural gas plant liquids production:

- Texas ( 40 percent)

- New Mexico (11 percent)

- Oklahoma (10 percent)

- Gulf of Mexico Federal Offshore (10 percent)

- Utah and Wyoming (7 percent)

- Alaska (5 percent).

Natural gas processing plants are usually located in the same general area where the natural gas is produced. Table E4 in Appendix $E$ lists the volumes of natural gas produced and processed in the same State, and the volumes of liquids extracted.

\section{Lease Condensate}

\section{Proved Reserves}

Proved reserves of lease condensate in the United States were 1,341 million barrels in 1997 (Table 16). This was 34 million barrels or 3 percent higher than in
1996. The reserves of four areas account for about 81 percent of the Nation's lease condensate proved reserves.

Percent of U.S.

Area Condensate Reserves

$\begin{array}{lr}\text { Gulf of Mexico Federal Oftshore } & 39 \\ \text { Texas } & 23 \\ \text { Louisiana } & 13 \\ \text { Ulah-Wyoming } & 6 \\ \text { Area Total } & 81\end{array}$

\section{Production}

Production of lease condensate was 174 million barrels, an increase of 12 million bartels, or 7 peroent, in 1997. The production of four areas account for about 86 percent of the Nation's lease condenșate production.

- Gulf of Mexico Federal Offshore (40 percent)

- Texas (24 percent)

- Louisiana (17 percent)

- Utah-Wyoming (5 percent).

\section{Reserves in Nonproduclng Reservoirs}

Like crude oil and natural gas, not all lease condensate proved reserves were contained in reservoirs that were producing during 1997. Proved reserves of 591 million barrels of lease condersate, an increase of 1 percent from 1996, were reported in nonproducing reservoirs in 1997. These reserves were reported by Category I and Category II operators who collectively accounted for more than 97 percent of total lease condensate production. About 63 percent of the nomproducing lease condenșate reșerves were located in the Gulf of Mexico Federal Offshore. 



\section{References}

1. Energy Information Administration. February 1980. U.S. Crude OiI and Naturd Gas Reserves 1977 Artnukl Report. DOE/ELA-0216(77). Washington, DC.

2. Energy Information Administration. September 1980. U.S. Crude Oil and Natural Gas Reserves 1978 Annial Report. DOE/ELA-0216(78). Washington, DC.

3. Energy Information Administration. August 1981. U.S. Crude Oil, Natural Gas, and Natural Gas Liquids Reserves 1979 Annual Report. DOE/EIA-0216(79). Washington, DC.

4. Energy Information Administration. Oxtober 1981. U.S. Crude Oils Natural Gas, and Natural Gas Liguids Reserves 1980 Antrual Report. DOE/EIA-0216(80). Washington, DC.

5. Energy Information Administration. August 1982. W.S. Crude Od, Natuml Gas, and Natural Gas Liguids Reserves 1981 Annual Report. DOE/ELA-0216(81). Washington, DC.

6. Energy Information Administration. August 1983. U.S. Crude Oil, Natzral Gas, and Natural Gas Liquids Reșenves 1982 Annual Reporl. DOE/ELA-1216(82). Washington, DC.

7. Energy Information Administration. October 1984. U.S. Crude Oil, Natunal Gas, and Nafurd Gas Liquids Resertes 1983 Annual Report. DOE/ELA-0216(83). Washington, DC.

8. Energy Irformation Administration. October 1985. U.S. Crude Oil, Natural Gas, and Natural Gas Liquids Reserves 1984 Annual Report. DOE/ELA-0216(84). Washington, $D C$.

9. Energy Information Administration. October 1986. U.S. Crude Oil, Narural Gas, and Nafural Gas Liquids Reserues 1985 Annual Report. DOE/EIA-0216(85). Washington, $\mathrm{DC}$.

10. Energy Irformation Administration. October 1987. U.S. Crude Oil, Natural Gas, and Natural Gas Liquids Reserves 7986 Annual Report. DOE/ELA-0216(86). Washington, DC.
11. Energy Information Administration. October 1988. U.S. Crude Oil, Natural Guts, and Natural Gas Liquids Reserves 1987 Annual Reporl. DOE/ELA-0216(87). Waskington, DC.

12. Energy Information Administration. September 1989. U.S. Crude Oil, Natumal Gas, and Natural Gas Liquids Reserves 1988 Annual Report. DOE/ELA-0216(88). Washington, DC.

13. Energy Information Administration. October 1990. 4.S. Crude Oil, Natural Ges, and Natural Gas Liquids Reserves 1989 Annual Report. DOE/EIA-0216(89). Washington, DC.

14. Energy Information Administration. September 1991. U.S. Crude Oil, Natural Gas, and Notural Gas Liquids Reserves 1990 Annual Report. DOE/ELA-0216(90). Washington, $D C$

15. Energy Information Administration. November 1992. L.5. Crade Oil, Natural Gas, and Natural Gas Liquids Reserdes 1991 Annual Report. DOE/ELA-0216(91). Washington, DC.

16. Energy Information Administration. October 1993. U.S. Crude Oil, Natural Gas, and Natural Gas Liquids Reserves 1992 Anntual Report. DOE/ELA-0216(92). Washington, $D C$

17. Energy Information Administration. October 1994. U.S. Crude Oil, Notulyal Gas, and Natural Gas Liquids Reserves 1993 Annual Report. DOE/ELA-0216(93). Washington, $D C$.

18. Energy Information Administration. October 1995. U.S. Crude Oil, Natural Gas, and Natural Gas Liquids Resenves 1994 Annual Report. DOE/ELA-0216(94). Washington, DC.

19. Energy Information Administration. November 1996. U.S. Cnade Oil, Natioral Gas, and Natural Gas Liquids Reserves 1995 Annual Report. DOE/EIA-0216(95). Washington, DC.

20. Energy Information Adrninistration. December 1997. U.S. Crude Oil, Natural Gas, and Natural Gas Liquids Reserves 1996 Annual Report. DOE/ELA-02I6(96). Washington, DC. 
21. Energy Irformation Administration. Otober 1908. Pelroleum Marketing Monthly. DOE/EIA. 0380(98/10). Washington, DC.

22. Energy Information Administration. October 1908 . Natural Gas Monthly. DOE/EIA-0130(98/10). Washington, DC, pg. 16.

23. Amerjcan Petroleun lnstitute, American Gis Association, and Canadian Petroleum Assactation. Reserves of Crude Oil, Natural Gas Liquids, and Nainral Gas in the United States and Canada as of December 31, 1979. Vol. 34. Jure 1980. Washington, DC.

24. Energy Irformation Administration, August 1990. U.S. Oit and Gas Reserves by Year of Field Discowery. DOE/EIA-0534. Washington, DC.

25. Oil \& Gas joumal. December 29, 1997. "Worldwide Look and Reserves and Production". Vol. 95, No. 52. Tulsa, OK, p. 24.

26. World Oil. August 1998. "World Trends". Houston, TX, P. 29.

27. World Oil. August 1998, "South America". Houston, $\mathrm{TX}, \mathrm{pp}, 45-47$.

28. Energy Information Administration. July 1998. 1997 Antua! Energy Reoiew. DOE/EIA-0384(97). Washington, DC, p. 39.

29. Energy Information Administration. July 1998. 1997 Annual Energy Review. DOE/ELA-0384(97). Washington, DC, p. 159.

30. Exxon Corporation, Irving, TX. Press Releas?, April 8, 1997.

31. Shell Exploration and Production Company. Houston, TX. Http://www.shellus,con, download of Europa Fact Sheet.
32. Shell Exploration and Production Company. Houston, TX. Http://www.shellus.com, dowrload of Macaroni Fact Sheet.

33. Amoco Corporation, Chicago, IL. Press Release, January $29,1997$.

34. Texaco Incorporated. White Plains, NY. 1997 Arroual Report. pp.14-15.

35. Atlantic Richtield Company. Los Angeles, CA. 1997 Annual Report. p. 5.

36. Shell Exploration and Production Company. Houston, TX. Http://www shelluss.som, download of Mensa Fact Sheet.

37. Texaco Incorporated. White Plains, NX. 1997 Arnual Report. P.17.

38. Louisiana Land and Exploration Company, New Orleans, LA Press Release, March 19, 1997.

39. HS Resources, Incorpated. San Francisco, CA. 1997 Annual Report. P. 9.

40. U.S. Department of Energy. DOE This Month. Vol. 15. No. 8. Washington, DC, August 1992- p. 13.

41. U.S. Department of the Interior. 1995. National Assessment of the United States Oil and Gas Resources. U.S. Geological Survey Circular 1118. Denver, CO, Pp. $5-6$.

42. U.\$. Department of the Interior, Minerals Management Service, Gulf of Mexico OCS Regional Office. August 1995. Estimated Proved Oil and Gas Reserves, Guif of Mexico, December 31, 1994. OCS Report MMS 94-0045. New Orleans, LA. PP. 5-6. 
Appendix A

Operator Data by Size Class 



\section{Operator Data by Size Class}

To remain competitive in the domestic oil and gas industry, companies have to reduce costs and look for areas of profitable growth. Over the past few years, we have seen companies restructure to focus on their core areas of profit. This restructuring has taken many forms, for example, laying off employees, early retirements and buyouts, flattening management structure, selective sales of marginally profitable properties, and acquisitions. Documenting some of these changes is important. Appendix $\mathrm{A}$ is a series of tables of the proved reserves and production by production size dass for the years 1992 through 1997 for oil and gas well operators. The tables show the volumetric change and percent change from the previous year and from 1992. In addition they show the 1997 average per operator in each class. All companies that reported to EJA were ranked by production size for each of the 6 years. We computed company production size classes as the sum of the barrel ojl equivalent of the crude oil production, lease condersate production, and wet gas production for each operator. The companies were then placed in the following production size classes: 1-10, 11-20, 21-100, 101-500, and all "other" oil and gas operators. The "other" category contains 22,178 small operators. We estimate production and teserves for small operatots each year from a sample of approximately 8 percent of these operators.

Class 1-10 contains the 10 highest producing comparies each year on a barrel oil equivalent basis. These companies are not necessarily the same 10 companies each $y$ ear.

We also include statistics for operator Category sizes at the bottom portion of tables in this appendix. These are the categories used by EIA in processing and assessing reserves surveys and are presented here as additional perspective. For further explanation of categories sizes see definitions and descriptions in Appendix $\mathrm{E}$.

\section{Natural Gas}

\section{Proved Reserves}

The wet natural gas proved reserves reported for 1992 through 1997 have changed from 173.3 tritlion cubic feet to 175.7 trillion cubic feet (Table A1). Reserves have been increasing for the last five yeats in a row and are above the 1992 level. These proved reserves are highly concentrated in the larger companies. In 1997, the top 20 (Class 1-10 and Class 11-20) producing companies had 55 percent of the proved reserves of natural gas. The next two size classes contain 80 and 400 companies and account for 26 and 13 percent of the U.S. natural gas proved reserves, respectively. The top 20 operators had a decline of 6 percent in their natural gas proved reserves from 1992 to 1997. While the rest of the operators (Class 21-100, Class 101-500, and Class Other) had an increase of 12 percent. In 1997, the top 20 operators' natural gas reserves increased by 15 percent from 1996.

\section{Production}

Wet natural gas production has slightly increased from 19.9 trillion cubic feet to 20.1 trillion cubic feet in 1997 (Table A2). In 1997, the top 20 producing companies had 52 percent of the production of wet natural gas, while having 55 percent of the proved reserves. The next two size classes have 28 and 13 percent of the wet natural gas production, respectively. The top 20 operators had an increase of 8 percent in their wet natural gas production from 1992 to 1997. The rest of the operators had an increase of 12 percent from 1992 to 1997. The top 20 operators' wet natural gas production had an decrease of 5 percent in 1997, while the rest of the operators remained the same. 


\section{Crude OII}

\section{Proved Reserves}

Proved reserves of crude oil are more highly concentrated in a few companies than those of natural gas. The 20 largest oil and gas producing companies in 1997 had 64 percent of $U S$. proved reserves of crude oil (Table A3), in contrast to wet natural gas where these same companies operated only 55 percent of the total proved reserves. These companies have tended in the past few years to concentrate their domestic operations in fewer fields and focus more of their resources on their foreign operations.

U.S. proved reserves of crude oil increased 2.4 percent in 1997 . The top 20 producing companies had a decline of 6 petcent in their domestic proved reserves of crude oil during 1997. The top 20 class had a decline of 20 percent in their crude oil proved reserves from 1992 to 1997. The next two size classes each account for 9 percent of the US. crude oil proved reserves. The class "other" had a 15 percent decrease from 1992 to $199 \%$. During the 1992-1997 period, many operators were continuing to actively buy, selt, and restructure their on property positions.

\section{Production}

Crude oil production reported for 1992 through 1997 has decreased from 2.4 billion barrels to 2.1 billion barrels (Table A4). The 20 largest oil and gas producing companies had 61 percent of U.S. production of crude oil in 1997, while in 1992 they accounted for 69 percent of production. This is in contrast to wet natural gas where these same comparies prodused only 52 percent of the total.

U.S. production of crude oil declined by 13 percent from 1992 to 1997 . The top 20 operators had a decline of 22 percent in their oil production during the same periad. U.S. production of crude oil dectined by 2 percent from 1996 to 1997 , while the top 20 operators production decreased by 7 percent. The next two size classes account for 17 and 11 percent of the U.S. crude oil production, respectively.

\section{Fields}

The number of fields in which Category I and Category Il operators were active dropped significantly during the 1992-1997 period. Fields in which these large operators were active dropped by 2,227 or 8 percent (Table A5). Most of the changes in operator field counts resulted from the top 20 operators class concentrating their effort in a diminishing number of fields. From 1992 through 1997, the rumber of fields in which the top 20 operators were active in dropped by 2,798 or 37 percent, while in 1997 the number dropped 8 percent from 1996. 
Table A1. Natural Gas Proved Reserves, Wet After Lease Separation, by Operalor Production Size Class, 1992-1997 (Bjtion Cubic Feet at 14.73 psia and $60^{\circ}$ Fahrenhelt)

\begin{tabular}{|c|c|c|c|c|c|c|c|c|c|}
\hline Size Clase & 1992 & 1993 & 1994 & 1095 & 1996 & 1997 & $\begin{array}{l}1996-1997 \\
\text { Volumi and } \\
\text { Percent } \\
\text { Change }\end{array}$ & $\begin{array}{l}\text { 19B2-1997 } \\
\text { Volunt and } \\
\text { Percent } \\
\text { Chang? }\end{array}$ & $\begin{array}{c}1997 \\
\text { Average } \\
\text { Poterves } \\
\text { por Operator }\end{array}$ \\
\hline $\begin{array}{l}\text { Class 1-10 } \\
\text { Percent of Tolal }\end{array}$ & $\begin{array}{r}74,350 \\
42.9 \%\end{array}$ & $\begin{array}{l}77.552 \\
\quad 45.5 \%\end{array}$ & $\begin{array}{l}76,665 \\
44.6 \%\end{array}$ & $\begin{array}{r}75,856 \\
43.7 \%\end{array}$ & $\begin{array}{r}72,606 \\
41.5 \%\end{array}$ & $\begin{array}{l}68.876 \\
39.2 \%\end{array}$ & $\begin{array}{r}-3,730 \\
-5.1 \%\end{array}$ & $\begin{array}{r}-5,474 \\
.7 .4 \%\end{array}$ & $6,887,600$ \\
\hline $\begin{array}{l}\text { Class 11-20 } \\
\text { Percent of Tolal }\end{array}$ & $\begin{array}{l}26,442 \\
16.4 \%\end{array}$ & $\begin{array}{l}22,467 \\
13,2 \%\end{array}$ & $\begin{array}{l}22,691 \\
13,2 \%\end{array}$ & $\begin{array}{r}24,648 \\
14.2 \%\end{array}$ & $\begin{array}{l}25,416 \\
14.5 \%\end{array}$ & $\begin{array}{l}27.705 \\
15.9 \%\end{array}$ & $\begin{array}{l}2,289 \\
9.0 \%\end{array}$ & $\begin{array}{l}-737 \\
-2.6 \%\end{array}$ & $2,770.500$ \\
\hline $\begin{array}{l}\text { Claes 21-100 } \\
\text { Percent of Todel }\end{array}$ & $\begin{array}{l}38,398 \\
22.2 \%\end{array}$ & $\begin{array}{l}39.135 \\
23.0 \%\end{array}$ & $\begin{array}{l}40,566 \\
23.6 \%\end{array}$ & $\begin{array}{l}42,604 \\
24.6 \%\end{array}$ & $\begin{array}{l}43,900 \\
24.7 \%\end{array}$ & $\begin{array}{l}45.593 \\
25.9 \%\end{array}$ & $\begin{array}{l}2,293 \\
5.3 \%\end{array}$ & $\begin{array}{l}7,205 \\
19.8 \%\end{array}$ & 569.913 \\
\hline $\begin{array}{l}\text { Class } 101-500 \\
\text { Percent of Total }\end{array}$ & $\begin{array}{l}19,728 \\
11.4 \%\end{array}$ & $\begin{array}{l}19,870 \\
11.7 \%\end{array}$ & $\begin{array}{l}20,608 \\
12,0 \%\end{array}$ & $\begin{array}{l}20.150 \\
\quad 11.6 \%\end{array}$ & $\begin{array}{l}23.483 \\
12.8 \%\end{array}$ & $\begin{array}{r}23,338 \\
13.3 \%\end{array}$ & $\begin{array}{l}655 \\
3.8 \%\end{array}$ & $\begin{array}{l}3,610 \\
18.3 \%\end{array}$ & 58.345 \\
\hline $\begin{array}{l}\text { Class Olher (22,178) } \\
\text { Percent of Total }\end{array}$ & $\begin{array}{r}12,401 \\
7,2 \%\end{array}$ & $\begin{array}{l}11,466 \\
6.7 \%\end{array}$ & $\begin{array}{r}11.409 \\
6.6 \%\end{array}$ & $\begin{array}{l}10,218 \\
5.9 \%\end{array}$ & $\begin{array}{r}11,342 \\
6.5 \%\end{array}$ & $\begin{array}{l}10,209 \\
5.6 \%\end{array}$ & $\begin{array}{l}-1,133 \\
-10.0 \%\end{array}$ & $\begin{array}{l}-2,192 \\
-17.7 \%\end{array}$ & 0.460 \\
\hline $\begin{array}{l}\text { Category I (169) } \\
\text { Percenl ol Totel }\end{array}$ & $\begin{array}{r}144,351 \\
83.3 \%\end{array}$ & $\begin{array}{r}742,892 \\
83.8 \%\end{array}$ & $\begin{array}{r}143.703 \\
83.6 \%\end{array}$ & $\begin{array}{r}148,293 \\
85.4 \%\end{array}$ & $\begin{array}{r}146,601 \\
83.7 \%\end{array}$ & $\begin{array}{r}147.491 \\
83.9 \%\end{array}$ & $\begin{array}{l}090 \\
0.6 \%\end{array}$ & $\begin{array}{l}3.140 \\
2.2 \%\end{array}$ & $872.72 d$ \\
\hline $\begin{array}{l}\text { Category II (418) } \\
\text { Percent ol Totel }\end{array}$ & $\begin{array}{l}17,662 \\
10.2 \%\end{array}$ & $\begin{array}{l}17,305 \\
10.2 \%\end{array}$ & $\begin{array}{l}18.159 \\
10.6 \%\end{array}$ & $\begin{array}{l}15.828 \\
9.1 \%\end{array}$ & $\begin{array}{l}18,382 \\
10.5 \%\end{array}$ & $\begin{array}{l}17.764 \\
10.1 \%\end{array}$ & $\begin{array}{l}-618 \\
-3.4 \%\end{array}$ & $\begin{array}{l}82 \\
0.5 \%\end{array}$ & 42.498 \\
\hline $\begin{array}{l}\text { Category Ill }\{22,037) \\
\text { Percent of Jotal }\end{array}$ & $\begin{array}{r}11.276 \\
6.5 \%\end{array}$ & $\begin{array}{r}10,292 \\
6,0 \%\end{array}$ & $\begin{array}{l}10,078 \\
5.9 \%\end{array}$ & $\begin{array}{r}9,416 \\
5,4 \%\end{array}$ & $\begin{array}{l}10,164 \\
5.8 \%\end{array}$ & $\begin{array}{l}10,466 \\
6.0 \%\end{array}$ & $\begin{array}{l}302 \\
3.0 \%\end{array}$ & $\begin{array}{l}.810 \\
-7.2 \%\end{array}$ & 0.475 \\
\hline $\begin{array}{l}\text { Total Publlshed } \\
\text { Percent of Totad }\end{array}$ & $\begin{array}{l}173,3 \times 9 \\
100,0 \%\end{array}$ & $\begin{array}{l}170,490 \\
100.0 \%\end{array}$ & 171,939 & $\begin{array}{l}173,476 \\
100.0 \%\end{array}$ & $\begin{array}{r}175,147 \\
100.0 \%\end{array}$ & $\begin{array}{l}176,721 \\
100.0 \%\end{array}$ & $\begin{array}{l}574 \\
0.3 \times\end{array}$ & $\begin{array}{r}2,412 \\
1.4 \%\end{array}$ & 7.740 \\
\hline
\end{tabular}

Note: There were 22,037 achwe Gategory III operators In the 1997 sample frame. The reserves and producllon of Category ill operators were estimatied trom an adjusted sample of 2,494 Category lil operators (Table E2). The "other" size class tepresents 22,178 operators in the 1997 frane (22, 678 acthe operators minus the 500 largesi operators).

Source: Energy Information Administration, Otfice of Oil and Gas. 
Table A2. Naturel Gas Production, Wet After Lease Separation, by Operator Production Size Class, 1992-1997

(Billion Cubic Feet at $14.73 \mathrm{psia}$ and $60^{\circ}$ Fahrenhelt)

\begin{tabular}{|c|c|c|c|c|c|c|c|c|c|}
\hline Size Clasg & 1992 & 1993 & 1994 & $19 \dot{15}$ & 1996 & 1997 & $\begin{array}{l}\text { 1996-1ba7 } \\
\text { Volume and } \\
\text { Percent } \\
\text { Change }\end{array}$ & $\begin{array}{c}1992-1997 \\
\text { Volume and } \\
\text { Pereent } \\
\text { Chainge }\end{array}$ & $\begin{array}{c}1997 \\
\text { Average } \\
\text { production } \\
\text { per Operaliox }\end{array}$ \\
\hline $\begin{array}{l}\text { Clase 1-10 } \\
\text { Percent of Toral }\end{array}$ & $\begin{array}{l}6,625 \\
36.3 \%\end{array}$ & $\begin{array}{l}6,801 \\
36.5 \%\end{array}$ & $\begin{array}{l}7.216 \\
37.6 \%\end{array}$ & $\begin{array}{l}7,174 \\
38.0 \%\end{array}$ & $\begin{array}{l}7,448 \\
37,5 \%\end{array}$ & $\begin{array}{l}7,178 \\
35.7 \%\end{array}$ & $\begin{array}{l}-270 \\
-3.6 \%\end{array}$ & $\begin{array}{l}553 \\
8.3 \%\end{array}$ & 717.800 \\
\hline $\begin{array}{l}\text { Cless 11-20 } \\
\text { Percent of Tolet }\end{array}$ & $\begin{array}{l}3.036 \\
+5.6 \%\end{array}$ & $\begin{array}{l}2,851 \\
15,3 \%\end{array}$ & $\begin{array}{l}3,083 \\
18.0 \%\end{array}$ & $\begin{array}{l}3.101 \\
1 / 5.4 \%\end{array}$ & $\begin{array}{l}3,002 \\
15.1 \%\end{array}$ & $\begin{array}{l}3,286 \\
16.3 \%\end{array}$ & $\begin{array}{l}284 \\
9.5 \%\end{array}$ & $\begin{array}{l}250 \\
8.2 \%\end{array}$ & 328.600 \\
\hline $\begin{array}{l}\text { Class 21-100 } \\
\text { Percent of Total }\end{array}$ & $\begin{array}{r}4,592 \\
25.1 \%\end{array}$ & $\begin{array}{l}4,694 \\
26.3 \%\end{array}$ & $\begin{array}{l}4,878 \\
25.4 \%\end{array}$ & $\begin{array}{l}4, \mathrm{e} 71 \\
2.5 .8 \%\end{array}$ & $\begin{array}{l}5.316 \\
26.7 \%\end{array}$ & $\begin{array}{l}5.729 \\
28.4 \%\end{array}$ & $\begin{array}{l}413 \\
7.8 \%\end{array}$ & $\begin{array}{l}1.137 \\
24.9 \%\end{array}$ & 71.813 \\
\hline $\begin{array}{l}\text { Clase 101-500 } \\
\text { Percent ol Total }\end{array}$ & $\begin{array}{l}2,411 \\
13.2 \%\end{array}$ & $\begin{array}{l}2,597 \\
13.9 \%\end{array}$ & $\begin{array}{l}2,552 \\
13.3 \%\end{array}$ & $\begin{array}{l}2,477 \\
13.1 \%\end{array}$ & $\begin{array}{l}2,623 \\
13.2 \%\end{array}$ & $\begin{array}{l}2,665 \\
\$ 32 \%\end{array}$ & $\begin{array}{l}42 \\
1.6 \%\end{array}$ & $\begin{array}{l}254 \\
10.5 \%\end{array}$ & 6.663 \\
\hline $\begin{array}{c}\text { Clase Ohinr }[22,178) \\
\text { Porcent al Tolal }\end{array}$ & $\begin{array}{l}1,605 \\
8.8 \%\end{array}$ & $\begin{array}{l}1,4 \mathrm{BB} \\
8.0 \%\end{array}$ & $\begin{array}{l}1,481 \\
7,7 \%\end{array}$ & $\begin{array}{l}1,251 \\
1) .6 \%\end{array}$ & $\begin{array}{l}1,494 \\
7.5 \%\end{array}$ & $\begin{array}{l}1,276 \\
8.3 \%\end{array}$ & $\begin{array}{l}-20 \mathrm{~B} \\
-14.0 \%\end{array}$ & $\begin{array}{l}-329 \\
-20.5 \%\end{array}$ & 0.058 \\
\hline $\begin{array}{l}\text { Cetegory II (418) } \\
\text { Percent of Total }\end{array}$ & $\begin{array}{l}2,036 \\
11.1 \%\end{array}$ & $\begin{array}{l}2,159 \\
11.6 \%\end{array}$ & $\begin{array}{l}2,221 \\
11.6 \%\end{array}$ & $\begin{array}{l}1,923 \\
10.2 \%\end{array}$ & $\begin{array}{l}2,129 \\
10.7 \%\end{array}$ & $\begin{array}{l}1,979 \\
9.8 \%\end{array}$ & $\begin{array}{l}-149 \\
-7.0 \%\end{array}$ & $\begin{array}{l}-57 \\
-2.8 \%\end{array}$ & 4.734 \\
\hline $\begin{array}{c}\text { Category if }(22,037) \\
\text { Percent of Total }\end{array}$ & $\begin{array}{l}1,466 \\
8.0 \%\end{array}$ & $\begin{array}{l}1,360 \\
7.3 \%\end{array}$ & $\begin{array}{l}1,3933 \\
0.9 \%\end{array}$ & $\begin{array}{l}1,131 \\
\text { ti.1\% }\end{array}$ & $\begin{array}{l}1,304 \\
6.7 \%\end{array}$ & $\begin{array}{l}1,258 \\
62 \%\end{array}$ & $\begin{array}{l}-106 \\
-7.8-\%\end{array}$ & $\begin{array}{l}-208 \\
-14.2 \%\end{array}$ & 0.057 \\
\hline $\begin{array}{l}\text { Total Published } \\
\text { Purcent of Total }\end{array}$ & $\begin{array}{c}18,269 \\
100,0 \%\end{array}$ & $\begin{array}{c}18,641 \\
100.0 \%\end{array}$ & $\begin{array}{c}19,210 \\
100,0 \%\end{array}$ & $\begin{array}{c}18,874 \\
100,0 \%\end{array}$ & $\begin{array}{c}19,873 \\
100.0^{\%}\end{array}$ & $\begin{array}{c}20,134 \\
100.0 x\end{array}$ & $\begin{array}{l}261 \\
1.3 \%\end{array}$ & $\begin{array}{c}1,665 \\
10.2 \%\end{array}$ & 0.888 \\
\hline
\end{tabular}

Note: There mere 2z, 037 active Category III operators in the 1697 gample frame. The foserves and production of Category lil operators were estimated trom an adjusted sample of 2,494 Category ill ope1ators (Table E2). The "other' size class represenis 22, 178 operators in ine 1997 frame (22.679 acthe operators minus the 5 too largest cperalars).

Source; Enercy Informuation Administralion, Office of Oil and Gas. 
Table A3. Crude Oil Proved Reserves by Operator Production Size Class, 1992-1997 (Million Barrels of 42 U.S. Gallons)

\begin{tabular}{|c|c|c|c|c|c|c|c|c|c|}
\hline Size Class & 1992 & 1993 & 1904 & 1905 & 1996 & 1997 & $\begin{array}{l}1995-1997 \\
\text { volume and } \\
\text { Proent } \\
\text { Change }\end{array}$ & $\begin{array}{l}1992-1907 \\
\text { Volume and } \\
\text { Percent } \\
\text { Change }\end{array}$ & $\begin{array}{c}1997 \\
\text { Average } \\
\text { Reperves } \\
\text { par Opartor }\end{array}$ \\
\hline $\begin{array}{l}\text { Cleass } t-10 \\
\text { Fercent of Total }\end{array}$ & $\begin{array}{l}15,733 \\
66.3 \%\end{array}$ & $\begin{array}{r}14,894 \\
64,9 \%\end{array}$ & $\begin{array}{l}14,351 \\
69.9 \%\end{array}$ & $\begin{array}{l}13,69 \uparrow \\
62.1 \%\end{array}$ & $\begin{array}{l}13,362 \\
\quad 60.7 \%\end{array}$ & $\begin{array}{l}11,434 \\
50.7 \%\end{array}$ & $\begin{array}{l}-1,926 \\
-14.4 \%\end{array}$ & $\begin{array}{l}-4,299 \\
.27 .3 \%\end{array}$ & $1,143.400$ \\
\hline $\begin{array}{l}\text { Class 11-20 } \\
\text { Pertent of Totel }\end{array}$ & $\begin{array}{r}2,250 \\
9.5 \%\end{array}$ & $\begin{array}{l}2,389 \\
10.4 \%\end{array}$ & $\begin{array}{l}2,276 \\
10.1 \%\end{array}$ & $\begin{array}{l}2.422 \\
10.8 \%\end{array}$ & $\begin{array}{l}2,013 \\
9.1 \%\end{array}$ & $\begin{array}{l}2.977 \\
13.2 \%\end{array}$ & $\begin{array}{l}964 \\
47.9 \%\end{array}$ & $\begin{array}{l}727 \\
32.3 \%\end{array}$ & 297.700 \\
\hline $\begin{array}{l}\text { Class 21-100 } \\
\text { Percent of Total }\end{array}$ & $\begin{array}{l}2,370 \\
10,0 \%\end{array}$ & $\begin{array}{l}2,401 \\
10,5 \%\end{array}$ & $\begin{array}{l}2,807 \\
11.6 \%\end{array}$ & $\frac{2,109}{11.7 \%}$ & $\begin{array}{l}3,155 \\
14,3 \%\end{array}$ & $\begin{array}{l}4,384 \\
19,4 \%\end{array}$ & $\begin{array}{l}1,229 \\
39.0 \%\end{array}$ & $\begin{array}{l}2,014 \\
85.0 \%\end{array}$ & 54.800 \\
\hline $\begin{array}{l}\text { Class 101-500 } \\
\text { Percenl of Total }\end{array}$ & $\begin{array}{l}1.463 \\
8.2 \%\end{array}$ & $\begin{array}{l}1.440 \\
6.3 \%\end{array}$ & $\begin{array}{l}1,512 \\
6.7 \%\end{array}$ & $\begin{array}{l}1.793 \\
\mathbf{B . 0 \%}\end{array}$ & $\begin{array}{r}1,838 \\
8.3 \%\end{array}$ & $\begin{array}{l}2,111 \\
9.4 \%\end{array}$ & $\begin{array}{l}273 \\
14.9 \%\end{array}$ & $\begin{array}{l}648 \\
44.3 \%\end{array}$ & 5.278 \\
\hline $\begin{array}{c}\text { Class Oiner }[22,178] \\
\text { Percent of Total }\end{array}$ & $\begin{array}{l}1,929 \\
0.1 \%\end{array}$ & $\begin{array}{l}1,8333 \\
8.0 \%\end{array}$ & $\begin{array}{c}1,711 \\
7.6 \%\end{array}$ & $\begin{array}{l}1, \text { 㿟之 } \\
7.3 \%\end{array}$ & $\begin{array}{l}1,649 \\
7.5 \%\end{array}$ & $\begin{array}{l}1.640 \\
7.3 \%\end{array}$ & $-0.5 \%$ & $\begin{array}{l}-289 \\
-15.0 \%\end{array}$ & 0.074 \\
\hline $\begin{array}{l}\text { Category I (169) } \\
\text { Percent of Total }\end{array}$ & $\begin{array}{l}20,787 \\
87.5 \%\end{array}$ & $\begin{array}{l}20,090 \\
07.5 \%\end{array}$ & $\begin{array}{l}19,848 \\
\text { 6.7.5\% }\end{array}$ & $\begin{array}{l}19,647 \\
\quad 37.9 \%\end{array}$ & $\begin{array}{l}19,312 \\
87,7 \%\end{array}$ & $\begin{array}{r}19,461 \\
86.3\end{array}$ & $\begin{array}{l}149 \\
0.8 \%\end{array}$ & $\begin{array}{r}-1,306 \\
-6.3 \%\end{array}$ & 115.154 \\
\hline $\begin{array}{l}\text { Cetegony l| (418) } \\
\text { Perceln of T면. }\end{array}$ & $\begin{array}{l}1,750 \\
4,8 \%\end{array}$ & $\begin{array}{l}1,131 \\
4.9 \%\end{array}$ & $\begin{array}{l}1,142 \\
5.1 \%\end{array}$ & $\begin{array}{l}1,103 \\
4.9 \%\end{array}$ & $\begin{array}{l}1.117 \\
5.1 \%\end{array}$ & $\begin{array}{r}1,400 \\
6.2\end{array}$ & $\begin{array}{l}283 \\
25.3 \%\end{array}$ & $\begin{array}{l}250 \\
21.7 \%\end{array}$ & 3.349 \\
\hline $\begin{array}{c}\text { Category ill }(22,037) \\
\text { Porcenl of Tolal }\end{array}$ & $\begin{array}{r}1,823 \\
7.7 \%\end{array}$ & $\begin{array}{r}1.737 \\
7.8 \%\end{array}$ & $\begin{array}{r}1.668 \\
7.4 \%\end{array}$ & $\begin{array}{l}1,600 \\
7.2 \%\end{array}$ & $\begin{array}{l}1,588 \\
7.2 \%\end{array}$ & $\begin{array}{r}1,685 \\
7.5\end{array}$ & $\begin{array}{l}97 \\
6.1 \%\end{array}$ & $\begin{array}{l}-959 \\
-5.0 \%\end{array}$ & 0.076 \\
\hline $\begin{array}{l}\text { Total Publlshod } \\
\text { Percent of Total }\end{array}$ & $\begin{array}{l}23,745 \\
100.0 \%\end{array}$ & $\begin{array}{l}22,957 \\
10000 \times\end{array}$ & 20,457 & 22,351 & $\begin{array}{l}28,017 \\
100,0 \%\end{array}$ & $\begin{array}{l}22,548 \\
100,0 \%\end{array}$ & $\begin{array}{l}529 \\
2.4 \% 6\end{array}$ & $-1,199$ & 0.994 \\
\hline
\end{tabular}

Note: There were 22,037 active Calegory III operaibrs in the 1997 sample frame. The reserves and production of Category lil operators were estingled fron an adjusted sample of 2,494 Category lil operators (Table E2). The "other" size class represents 22,178 operalors in Ihe

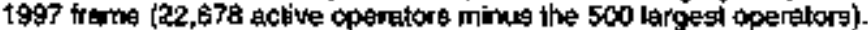

Solros: Energy Information Administration, Office of Oil and Gas. 
Table A4. Crude Oil Productlon by Operator Production Size Class, 1992-1997 (Miltion Barrels of 42 U.S. Gallons)

\begin{tabular}{|c|c|c|c|c|c|c|c|c|c|}
\hline Sire Ctass & 1902 & 1993 & 1994 & 1995 & $199 \%$ & 1997 & $\begin{array}{l}1994-1997 \\
\text { Volume and } \\
\text { Percant } \\
\text { Chonge }\end{array}$ & $\begin{array}{l}1992-1997 \\
\text { Volume and } \\
\text { Percent } \\
\text { Change }\end{array}$ & $\begin{array}{c}1997 \\
\text { Average } \\
\text { Production } \\
\text { per Operator }\end{array}$ \\
\hline $\begin{array}{l}\text { Class 1-10 } \\
\text { Percent ot Total }\end{array}$ & $\begin{array}{l}1,458 \\
58.6 \%\end{array}$ & $\begin{array}{l}1.346 \\
57.5 \%\end{array}$ & $\begin{array}{l}1,310 \\
\mathbf{5 7 . 8 \%}\end{array}$ & $\begin{array}{l}1,270 \\
5,77.4 \%\end{array}$ & $\begin{array}{l}1,220 \\
56.1 \%\end{array}$ & $\begin{array}{l}1,047 \\
49.0 \%\end{array}$ & $\begin{array}{l}-173 \\
-14.2 \%\end{array}$ & $\begin{array}{l}-411 \\
-28.2 \%\end{array}$ & 104.700 \\
\hline $\begin{array}{l}\text { Class 11-20 } \\
\text { Percent of Total }\end{array}$ & $\begin{array}{l}231 \\
9.4 \%\end{array}$ & $\begin{array}{l}236 \\
10.1 \%\end{array}$ & $\begin{array}{l}2.24 \\
9.9 \%\end{array}$ & $\begin{array}{l}221 \\
10.0 \%\end{array}$ & $\begin{array}{l}185 \\
8.5 \%\end{array}$ & $\begin{array}{l}202 \\
12.3 \%\end{array}$ & $\begin{array}{c}77 \\
41.6 \%\end{array}$ & $\begin{array}{c}31 \\
13.4 \%\end{array}$ & 26.200 \\
\hline $\begin{array}{l}\text { Class 21-100 } \\
\text { Percent of Totel }\end{array}$ & $\begin{array}{l}272 \\
11.1 \%\end{array}$ & $\begin{array}{l}278 \\
11.8 \%\end{array}$ & $\begin{array}{l}287 \\
12.7 \%\end{array}$ & $\begin{array}{l}276 \\
12.5 \%\end{array}$ & $\begin{array}{l}307 \\
14.1 \%\end{array}$ & $\begin{array}{l}373 \\
17.4 \%\end{array}$ & $\begin{array}{c}66 \\
21.5 \%\end{array}$ & $\begin{array}{l}101 \\
37.1 \%\end{array}$ & 4.863 \\
\hline $\begin{array}{l}\text { Class } 101-500 \\
\text { Percerm of Tolal }\end{array}$ & $\begin{array}{l}213 \\
8.7 \%\end{array}$ & $\begin{array}{l}202 \\
8.6 \%\end{array}$ & $\begin{array}{l}200 \\
6.8 \%\end{array}$ & $\begin{array}{l}: 214 \\
9.7 \%\end{array}$ & $\begin{array}{l}213 \\
9.8 \%\end{array}$ & $\begin{array}{l}237 \\
11.1 \%\end{array}$ & $\begin{array}{l}24 \\
11.3 \%\end{array}$ & $\begin{array}{c}24 \\
11.3 \%\end{array}$ & 0.593 \\
\hline $\begin{array}{c}\text { Class Other }\{22,178) \\
\text { Percent of Tolal }\end{array}$ & $\begin{array}{l}272 \\
11.1 \%\end{array}$ & $\begin{array}{l}279 \\
11.9 \%\end{array}$ & $\begin{array}{l}247 \\
10.9 \%\end{array}$ & $\begin{array}{l}232 \\
10.5 \%\end{array}$ & $\begin{array}{l}246 \\
11.4 \%\end{array}$ & $\begin{array}{l}219 \\
10.2 \%\end{array}$ & $\begin{array}{c}-29 \\
-11.7 \%\end{array}$ & $\begin{array}{c}-53 \\
-19.5 \%\end{array}$ & 0.010 \\
\hline $\begin{array}{l}\text { Category ! (169) } \\
\text { Percent of Total }\end{array}$ & $\begin{array}{l}2,022 \\
82.7 \%\end{array}$ & $\begin{array}{l}1.922 \\
82.2 \%\end{array}$ & $\begin{array}{l}1,87 \% \\
\$ 2.6 \%\end{array}$ & $\begin{array}{l}1, \sqrt{3444} \\
83.3 \%\end{array}$ & $\begin{array}{l}1,791 \\
82.4 \%\end{array}$ & $\begin{array}{l}1.760 \\
82.3 \%\end{array}$ & $\begin{array}{l}-31 \\
.1 .7 \%\end{array}$ & $\begin{array}{l}-262 \\
.13 .0 \%\end{array}$ & 10.414 \\
\hline $\begin{array}{l}\text { Categony if }\{418\} \\
\text { Percenl of Total }\end{array}$ & $\begin{array}{l}163 \\
6.7 \%\end{array}$ & $\begin{array}{l}153 \\
6.5 \%\end{array}$ & $\begin{array}{l}150 \\
6.6 \%\end{array}$ & $\begin{array}{l}130 \\
6.3 \% \%\end{array}$ & $\begin{array}{l}143 \\
B .6 \%\end{array}$ & $\begin{array}{l}157 \\
7,3 \%\end{array}$ & $\begin{array}{l}14 \\
9.9 \%\end{array}$ & $\begin{array}{l}-5 \\
-3.7 \%\end{array}$ & 0.376 \\
\hline $\begin{array}{l}\text { Category III }(22,037) \\
\text { Pencent of Total }\end{array}$ & $\begin{array}{l}261 \\
10.7 \%\end{array}$ & $\begin{array}{l}264 \\
11.3 \%\end{array}$ & $\begin{array}{l}239 \\
10.5 \%\end{array}$ & $\begin{array}{l}\% 30 \\
10.4 \%\end{array}$ & 230 & $\begin{array}{l}22.1 \\
10.3 \%\end{array}$ & $\begin{array}{l}-18 \\
.7 .5 \%\end{array}$ & $\begin{array}{c}-40 \\
.15 .3 \%\end{array}$ & 0.010 \\
\hline $\begin{array}{l}\text { Total Pubslaned } \\
\text { Purcent of Total }\end{array}$ & $\begin{array}{l}2,446 \\
100.0 \%\end{array}$ & $\begin{array}{l}2,339 \\
100.0 \%\end{array}$ & $\begin{array}{l}2,268 \\
100.0 \%\end{array}$ & $\begin{array}{l}2,713 \\
100.0 \%\end{array}$ & $\begin{array}{l}2,173 \\
100.0 \%\end{array}$ & $\begin{array}{l}2,138 \\
100.0 \%\end{array}$ & $\begin{array}{l}-35 \\
-1.6 \%\end{array}$ & $\begin{array}{c}-308 \\
-12.6 \%\end{array}$ & 0.094 \\
\hline
\end{tabular}

Nove: There were 22,037 active Calegory IIJ aperators in the 1997 sample frame. The resenves and proctuction ol Category III operalors

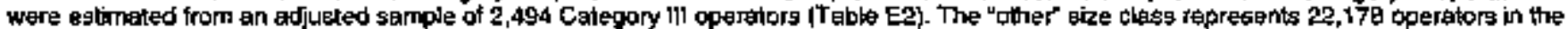
1997 frame (22,678 active operators minus the 500 largest operathrs).

Soures: Energy Infomation Administration, Office of Oil and Gas. 
Table A5. Operator Fivld Count by Operator Production Size Class, 1992-1997

\begin{tabular}{|c|c|c|c|c|c|c|c|c|c|}
\hline Siza Class & 1992 & 1993 & 1894 & 1998 & 1996 & 1997 & $\begin{array}{l}\text { 19ati-1997 } \\
\text { Number and } \\
\text { Poront } \\
\text { Chrorige }\end{array}$ & $\begin{array}{l}\text { 1992-1997 } \\
\text { Number and } \\
\text { Porcanl } \\
\text { Cheinge }\end{array}$ & $\begin{array}{l}1997 \text { Avartagt } \\
\text { Number of } \\
\text { Flolds por } \\
\text { Operator }\end{array}$ \\
\hline $\begin{array}{l}\text { Class 1-10 } \\
\text { Percent of Total }\end{array}$ & $\begin{array}{l}4.189 \\
14.7 \%\end{array}$ & $\begin{array}{l}3.591 \\
13.2 \%\end{array}$ & $\begin{array}{l}3,258 \\
12.2 \%\end{array}$ & $\begin{array}{l}3.113 \\
11,9 \%\end{array}$ & $\begin{array}{l}2, \mathrm{BOO} \\
10,7 \%\end{array}$ & $\begin{array}{l}2,586 \\
10.4 \%\end{array}$ & $\begin{array}{l}-234 \\
-0.4 \%\end{array}$ & $\begin{array}{c}-1,623 \\
-38,7 \%\end{array}$ & 257 \\
\hline $\begin{array}{l}\text { Class 11-20 } \\
\text { Percent ol Total }\end{array}$ & $\begin{array}{l}3.482 \\
12.1 \%\end{array}$ & $\begin{array}{l}2,998 \\
11.1 \%\end{array}$ & $\begin{array}{l}2,795 \\
10.5 \%\end{array}$ & $\begin{array}{l}2,772 \\
10.6 \%\end{array}$ & $\begin{array}{l}2,441 \\
9.3 \%\end{array}$ & $\begin{array}{r}2,257 \\
9.1 \%\end{array}$ & $\begin{array}{l}-184 \\
-7.5 \%\end{array}$ & $\begin{array}{l}-1,175 \\
-34.2 \%\end{array}$ & 226 \\
\hline $\begin{array}{l}\text { Class } 21-100 \\
\text { Percent of Totad }\end{array}$ & $\begin{array}{l}8,003 \\
28.2 \%\end{array}$ & $\begin{array}{l}7,600 \\
28.0 \%\end{array}$ & $\begin{array}{l}7,752 \\
29.1 \%\end{array}$ & $\begin{array}{l}7.589 \\
28.9 \%\end{array}$ & $\begin{array}{l}7.526 \\
28.7 \%\end{array}$ & $\begin{array}{l}7,159 \\
28.9 \%\end{array}$ & $\begin{array}{l}-367 \\
-4.9 \%\end{array}$ & $\begin{array}{l}-844 \\
-10.5 \%\end{array}$ & 89 \\
\hline $\begin{array}{l}\text { Class t01-500 } \\
\text { Percent of Total }\end{array}$ & $\begin{array}{r}11,896 \\
41.9 \%\end{array}$ & $\begin{array}{l}11,881 \\
43.9 \%\end{array}$ & $\begin{array}{c}11,078 \\
44.6 \%\end{array}$ & $\begin{array}{r}11,886 \\
45.4 \%\end{array}$ & $\begin{array}{l}12,492 \\
47.7 \%\end{array}$ & $\begin{array}{r}12,878 \\
52.0 \%\end{array}$ & $\begin{array}{l}386 \\
3.1 \%\end{array}$ & $\begin{array}{l}982 \\
8.3 \%\end{array}$ & 32 \\
\hline $\begin{array}{l}\text { Rest } \\
\text { Percent of Total }\end{array}$ & $\begin{array}{r}2,059 \\
7.2 \%\end{array}$ & $\begin{array}{l}1,715 \\
6.3 \%\end{array}$ & $\begin{array}{c}1,897 \\
7.1 \%\end{array}$ & $\begin{array}{l}1,601 \\
6.1 \%\end{array}$ & $\begin{array}{l}a_{952} \\
{ }_{3.6 \%}\end{array}$ & $\begin{array}{l}1,332 \\
5.4 \%\end{array}$ & $\begin{array}{l}380 \\
39.9 \%\end{array}$ & $\begin{array}{l}-727 \\
-35.3 \%\end{array}$ & 15 \\
\hline $\begin{array}{l}\text { Gategory I } \\
\text { Percent of Tolat }\end{array}$ & $\begin{array}{l}17,620 \\
62.0 \%\end{array}$ & $\begin{array}{l}16,6003 \\
61.2 \%\end{array}$ & $\begin{array}{l}15.161 \\
\quad 90.7 \%\end{array}$ & $\begin{array}{l}16,256 \\
62.1 \%\end{array}$ & $\begin{array}{r}15,635 \\
59.7 \%\end{array}$ & $\begin{array}{l}15,232 \\
58.2 \%\end{array}$ & $\begin{array}{l}-403 \\
-2.6 \%\end{array}$ & $\begin{array}{l}-2,38.8 \\
-13.6 \%\end{array}$ & 90 \\
\hline $\begin{array}{l}\text { Category ll } \\
\text { Parcent of Toles }\end{array}$ & $\begin{array}{l}10.790 \\
38.0 \%\end{array}$ & $\begin{array}{l}10.516 \\
38.8 \%\end{array}$ & $\begin{array}{l}10,457 \\
39.3 \%\end{array}$ & $\begin{array}{l}9,939 \\
37.9 \%\end{array}$ & $\begin{array}{r}10,576 \\
40.3 \%\end{array}$ & $\begin{array}{r}10,960 \\
41.8 \%\end{array}$ & $\begin{array}{l}384 \\
3.6 \%\end{array}$ & $\begin{array}{l}161 \\
1.5 \%\end{array}$ & 22 \\
\hline $\begin{array}{l}\text { Total Reported } \\
\text { Peroen Chargat }\end{array}$ & $\begin{array}{l}28,419 \\
100,0 \%\end{array}$ & $\begin{array}{l}27,119 \\
100,0 \%\end{array}$ & $\begin{array}{l}28,613 \\
100.0^{\circ}\end{array}$ & $\begin{array}{c}26,195 \\
100,0 \%\end{array}$ & $\begin{array}{l}26,211 \\
1000 \%\end{array}$ & $\begin{array}{c}26,192 \\
100.0 \%\end{array}$ & $\begin{array}{l}-19 \\
-0.1 \%\end{array}$ & $\begin{array}{r}-2,227 \\
-7,8 \%\end{array}$ & 39 \\
\hline
\end{tabular}

aThe reduced 1996 survey had fewer aperators and flekts in the "rest" class.

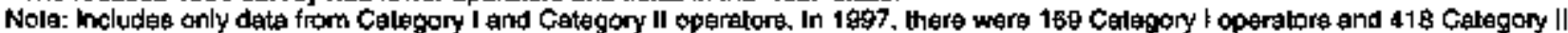
operators. The "resr' size class had 87 operators in 1997.

Source: Energy Intomeation Adrinistration, Dttice of Oil and Gas. 

Appendix B

Top 100 Oil and Gas Fields for 1997 



\section{Appendix B}

\section{Top 100 Oil and Gas Fields for 1997}

This appendix presents estimates of the proved reserves and production of the top 100 onl and gas fields. The oil field production and reserve data include both crude oil and lease condensate. The gas field production and reserve data is total wet natural gas (associated-dissolved plus nonassociated, wet after lease separation).

The top 100 oil fields in the United States as of December 31, 1997, had 14,940 million barrels of proved reserves (Table B1) accounting for 63 percent of the total United States (Table 6 and Table 16). Although there is considerable grouping of field-level statistics within the tables, rough orders of magnitude can be estimated for the proved reserves and production of most fjelds. Many fields in the top 100 groups are operated by only one or two operators, therefore, the totals for proved reserves are grouped as top 10, fop 20, top 50, and top 100 to avoid revealing company proprietary data. In the top 20 oil fields for 1997 there are three fields, Mississippi Canyon Blk 807 (Mars), Green Canyon Blk 244 (Troika), and Mississippi Canyon Blk 854 (Ursa) in the deep water of the Gulf of Mexico Federal Offshore. The top 100 oil fields in the United States as of December 3], 1997, had 1,176 million barrels of production (Table B1) or 51 percent of the total (Table 6 and Table 16). Many of the oil fields in the top 100 are very old, 47 oil fields were discovered prior to 1946. The oldest, Coalinga in California, was discovered in 1887. The fields with newer discovery dates are located in the Gulf of Mexico Offshore and Alaska.

The top 100 gas fjelds in the United States as of December 31, 1997, had 80,698 billion cubic feet of proved reserves (Table B2) or 46 percent of the total (Table 9). The top 100 gas fields in the United States as of December 31, 1997, had 6,563 bitlion cubic feet of production (Table B2) or 33 percent of the total (Table 9). Fewer of the gas fieids in the top 100 are very old. There were 19 gas fields that were discovered prior to 1946. The oldest, Big Sandy in Kentucky, was discovered in 1881. The fields with newer discovery dates are located in the Gulf of Mexico Offshore, south Texas and Virginia Several of the same fields are in both tables. 
Table B1. Top 100 U.S. Flelds Fanked by Oll Production within Proved Resenves Group, from Reported 1997 Field Level Deta"

(Million Barrels of 42 U.S. Gallons)

\begin{tabular}{|c|c|c|c|c|}
\hline Findd Neme & Loestion & $\begin{array}{l}\text { Discovery } \\
\text { Year }\end{array}$ & $\begin{array}{l}\text { Proved Reoervas } \\
\text { Renk Broup }\end{array}$ & $\begin{array}{l}\text { Reporkad Production } \\
\text { Aank Group }\end{array}$ \\
\hline Prudhoe Bay & AK & 1967 & 1.10 & 252.3 \\
\hline Kuparuk Fivyer & AK & 1969 & 1.10 & 96.1 \\
\hline Maldway-Sunset & $\mathrm{CA}$ & 1901 & $1-10$ & 61.6 \\
\hline Kan Fiver & $C_{A}$ & 1899 & $1 \cdot 10$ & 48.8 \\
\hline Belridge South & CA & $19+1$ & $1 \cdot 10$ & 41.1 \\
\hline Malssussippi Canyon Bak BO7 & GF & 1989 & 1.10 & 31.9 \\
\hline Yates & TX & 1928 & $1 \cdot 10$ & 20.9 \\
\hline 白k $\mathbf{H}$ 五ls & CA & 1919 & 1.10 & 20.5 \\
\hline Milne Point & AK & 1982 & 1.10 & 19.0 \\
\hline Wasson & $T X$ & 1937 & $\tau-10$ & 15.0 \\
\hline $\begin{array}{l}\text { Top } 10 \text { Volume Subtotal } \\
\text { Top } 10 \text { Percentage of U.s. Tota }\end{array}$ & & & $\begin{array}{r}0,001.3 \\
.33 .5 \% \\
\end{array}$ & $\begin{array}{l}607.1 \\
26.3 \%\end{array}$ \\
\hline Point Mcintyre & AK & 1988 & $11-20$ & 58.8 \\
\hline Spraberry Trend Area & $T X$ & 1950 & 11-20 & 22.1 \\
\hline Endleott & AK & 1978 & $11-20$ & 21.4 \\
\hline Wilmington & CA & 1932 & 11.20 & 19.5 \\
\hline Hondo & PF & 1969 & 11.20 & 16.3 \\
\hline Slaughtar & $T X$ & 1937 & 11.20 & 14.5 \\
\hline Levelland & $T X$ & 1945 & $11 \cdot 20$ & 9.4 \\
\hline San Ardo & CA & 1947 & $11 \cdot 20$ & 4.2 \\
\hline Green Canyon Bkx 244 & BF & 1994 & $11 \cdot 20$ & 1.1 \\
\hline Miesissippi Canyon B* 854 & GF & 1992 & $11 \cdot 20$ & 0.0 \\
\hline $\begin{array}{l}\text { Top } 20 \text { Yolume Subtotal } \\
\text { Top } 20 \text { Parentage of U.s. Total }\end{array}$ & & & $\begin{array}{r}10,140.9 \\
428 \% \\
\end{array}$ & $\begin{array}{l}774.5 \\
33.5 \% \\
\end{array}$ \\
\hline Garden Eanks Bk 426 & GF & 1997 & $21 \cdot 50$ & 282 \\
\hline Giddings & TX & 1960 & $21-50$ & 20.7 \\
\hline Easti Texas & $T X$ & 1930 & $21-50$ & 17.1 \\
\hline Vhosca Knoll Blk 980 & GF & 1981 & $21-50$ & 16.4 \\
\hline Pascado & PF & 1970 & 21.50 & 15.0 \\
\hline Cymric & $C A$ & 1916 & 21.50 & 14.0 \\
\hline Siminole & $T x$ & 1936 & 21.50 & 11.9 \\
\hline Niakuk & AK & 1984 & 21.50 & 10.5 \\
\hline Lost Hils & CA & 1910 & $21-50$ & 10.2 \\
\hline Coalinga & $\mathrm{CA}$ & 1887 & $21-50$ & $9 . \hat{6}$ \\
\hline Sall Creak & $T X$ & 1842 & 21.50 & 9.4 \\
\hline Courden North & $T X$ & 1930 & $21-50$ & 0.2 \\
\hline Say Manthand Bax 2 & GF \& LA & 1949 & $21-50$ & 8.6 \\
\hline Vácuum & NM & 1929 & $21-50$ & 8.0 \\
\hline Sho-Veltulm & OK & 1905 & $21-50$ & 7.4 \\
\hline Rangely & co & $190 \Omega$ & $21-50$ & 7.4 \\
\hline Ancelroy & $T x$ & 1926 & $21-60$ & 7.3 \\
\hline Fullerton & $T X$ & 1042 & $21-50$ & 7.0 \\
\hline Greater Angth & UT & 1956 & $21 \cdot 50$ & 6.0 \\
\hline Ventura & $\mathrm{GA}$ & 1916 & $21-50$ & 5.3 \\
\hline Goldsmith & $T x$ & 1985 & 21.50 & 4.3 \\
\hline Robertson North & $T x$ & 1956 & $21-50$ & 3.6 \\
\hline Hawkins & $T X$ & 1940 & $21 \cdot 50$ & $3 . \hat{2}$ \\
\hline Howard-Glasscock & $T x$ & 1925 & $21-50$ & 2.0 \\
\hline Eunice Monument & $N M$ & 1929 & $21-50$ & 1.3 \\
\hline Viosea Knall Ba 956 & GF & 1985 & $21-50$ & 0.9 \\
\hline Wasson 72 & $T x$ & 1940 & 21.50 & 0.0 \\
\hline East Breaks Blk 945 & GF & 1994 & $21-50$ & 0.0 \\
\hline Groen Caryan Blk 20S & GF & 1988 & $21-50$ & 0.0 \\
\hline Mississippi Canyon BK $\$ 35$ & GF & 1994 & $21-50$ & 0.0 \\
\hline $\begin{array}{l}\text { Top 5o Volume Suthtolel } \\
\text { Top 5D Porcentage of U.S. Totel }\end{array}$ & & & $\begin{array}{l}12,998.7 \\
54.3 \%\end{array}$ & $\begin{array}{r}1,019,9 \\
44,1 \% \\
\end{array}$ \\
\hline
\end{tabular}


Table E1. Top 100 U.S. Fields Ranked by Oil ${ }^{a}$ Production within Proved Roserves Group, from Reported 1997 Fleld Level Date" (Continued) (Minlion Barrets of $42 \mathrm{U.S.} \mathrm{Gallons)}$

\begin{tabular}{|c|c|c|c|c|}
\hline Ftad Name & Location & $\begin{array}{l}\text { OHgeovery } \\
\text { Year }\end{array}$ & $\begin{array}{l}\text { Praved Resirveo } \\
\text { Rank Croup }\end{array}$ & $\begin{array}{l}\text { Peported Produxilon } \\
\text { Rink Croup }\end{array}$ \\
\hline Ewing Bank Bdk Br3 & Gr & 1981 & 51.100 & 12.1 \\
\hline Eughone Islend Sa Bk 330 & CF & 1971 & $51-100$ & 9.5 \\
\hline Ship Shoal Blk 169 & CF & 1981 & $51-100$ & 7.2 \\
\hline Main Pass Sa Btk 299 & CF & 1967 & $51-100$ & 6.6 \\
\hline West Delta Bnk 30 & cF & 1949 & $51-100$ & 6.5 \\
\hline South Pass Bak 61 & GF & 1955 & $51-100$ & 6.5 \\
\hline Ceder Hills & ND & 1995 & $51-100$ & 6.1 \\
\hline Jay & FL \& AL & 1970 & 51.100 & 5.6 \\
\hline Wattenberg & $\infty$ & \pm 970 & $51-100$ & 5.6 \\
\hline South TImbaler Blk 52 & cF & 1950 & $51-100$ & 5.4 \\
\hline Viosca Knot Blk 826 & GE & 1988 & $51-100$ & 53 \\
\hline Oregon Bastr & WY & 1912 & $51-100$ & 4.4 \\
\hline Means & Tx & 1984 & $\$ 1-100$ & 4.2 \\
\hline Grayturg-Jeckson & NM & 1929 & $51-100$ & 4.0 \\
\hline Huntington Beach & CA & 1920 & 51.100 & 3.6 \\
\hline Priniar Reservoir East & $W Y$ & 1978 & $51-100$ & 3.5 \\
\hline Dollarnive & TX AN & 1945 & $51-700$ & 3.5 \\
\hline Shlp Snocal Sa Bk 349 & GF & 1994 & 51.100 & 3.3 \\
\hline Beta & PF & 1976 & 51.100 & 32 \\
\hline TXL & $T \times$ & 1944 & $51-100$ & 32 \\
\hline Monument & NMt \& UT & 1935 & 51.100 & 2.8 \\
\hline BAugbell & UT & 1949 & $51 \cdot 100$ & 2.7 \\
\hline Codar Lake & TX & 1938 & $51-100$ & 27 \\
\hline Dos Cuadras & PF & 1968 & 51.100 & 2.6 \\
\hline Stepheris County Regular & $T X$ & 1915 & $51-100$ & 2.5 \\
\hline Jo-N同 & $\mathbf{T X}$ & 1953 & 51.100 & 2.5 \\
\hline Inglewood & CA & 1924 & $51-100$ & 2.5 \\
\hline Mabee & $T X$ & $19 \mathrm{dd}$ & $51-100$ & 2.4 \\
\hline Ek Basin & WratT & 1915 & $51-100$ & 2.3 \\
\hline Hamiton Dome & $W Y$ & 1918 & 51.100 & 22 \\
\hline Sand HIls & TX & 1930 & $51-100$ & 2.1 \\
\hline Welch & Tx & 1942 & 51.100 & 2.1 \\
\hline Hantzog Draw & $W Y$ & 1976 & 51.100 & 2.1 \\
\hline Pennel & MT & 1905 & $51-100$ & 2.0 \\
\hline Postles & OK & 1958 & $51-100$ & 1.8 \\
\hline Cowden Soulh & TX & 1930 & $51-100$ & 1.7 \\
\hline Kam Front & CA & 1925 & 51.100 & 1.7 \\
\hline Cottonwood Creak & Wr & 1953 & $51-100$ & 1.5 \\
\hline Bovenly Hals & $\mathrm{CA}_{\mathrm{A}}$ & 1900 & $51-100$ & 1.4 \\
\hline Brealolinds & CA & 1897 & $\$ 1-100$ & 1.4 \\
\hline Hobbs & NM & 1928 & 51.100 & 1.1 \\
\hline Justls & NM & 1957 & $51-100$ & 1.0 \\
\hline Cote Blancho Bay West & LA & 1940 & 51.100 & 0.6 \\
\hline Gosegit Sounth & $T X$ & 1956 & $51-100$ & 0.5 \\
\hline Breon Canyon Blk 254 & GF & 1994 & $51-+00$ & 0.0 \\
\hline Graen Canyon Elk 159 & CF & 1902 & $51-100$ & 0.0 \\
\hline Viosca Knoll Elk 915 & GaF & 1093 & $\$ 1.100$ & 0.0 \\
\hline Viosca Knoll Bak 786 & GF & 1006 & $51-100$ & 0.0 \\
\hline Gardẹn Banks Bdk 260 & GF & 1995 & $51-100$ & 0.0 \\
\hline Ewing Bank E1k 921 & CF & 1998 & $51-100$ & 0.0 \\
\hline 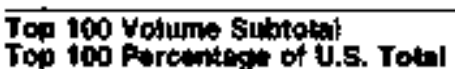 & & & $14,939.6 \%$ & $\begin{array}{l}1,175.5 \\
50.6 \%\end{array}$ \\
\hline
\end{tabular}

Anciudes lease cendansatis.

bDily $B 7$ percent of the estimated reserves were sunveyed by ElA at the fletd level toom large and intermedlate operailors.

Noles: Fieles art grouped in "proved roserves rank groups" and then listed within that group in descernding order by annuat production

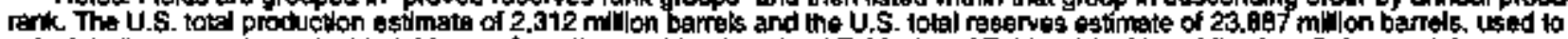

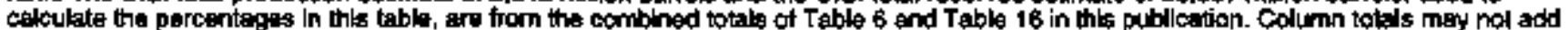
chlo to independent rounding

Source: Energy Information Adkinistration, Ofifee of Oll and Gas. 
Table B2. Top 100 U.S. Fields Ranked by Gas ${ }^{a}$ Production within Proved Reserves Group, from Reported 1997 Fiold Level Data'

(Eillion Cubic Feat)

\begin{tabular}{|c|c|c|c|c|}
\hline Fleild Namę & Location & $\begin{array}{c}\text { Oiscovery } \\
\text { Year. }\end{array}$ & $\begin{array}{l}\text { Proved Reserves } \\
\text { Rank Group } \\
\end{array}$ & $\begin{array}{l}\text { Reported Production } \\
\text { Rank Broup }\end{array}$ \\
\hline Basin & NM & 1947 & $1-10$ & 787.2 \\
\hline Blanco & NH \& $\mathrm{CO}$ & 1927 & $\uparrow-10$ & 605.9 \\
\hline Carthage & TX & 1936 & 1.10 & 211.7 \\
\hline Frudhoe Bxy & AK & 1967 & 1.10 & 207.8 \\
\hline Moblls Bay & Al & 1995 & $1-10$ & 132.9 \\
\hline Oakwood & VA & 1990 & $1 \cdot 10$ & 25.5 \\
\hline Bigg Piney-Labarge & $\mathbf{W Y}$ & 1964 & $1 \cdot 10$ & 9.7 \\
\hline $\begin{array}{l}\text { Top } 10 \text { Yoluma Subtotal } \\
\text { Top } 10 \text { Purcentage of U.S. Totar }\end{array}$ & & & $\begin{array}{r}37,405.0 \\
21,3 \%\end{array}$ & $\begin{array}{r}2,643.6 \\
13.1 \% \\
\end{array}$ \\
\hline Giddings & $\operatorname{TX}$ & 1660 & $1-20$ & 393.2 \\
\hline Cook Inleal North & AK & 1082 & $1-20$ & 52.0 \\
\hline Big Sandy & KY & 1881 & 1.20 & 49.0 \\
\hline Fogarty Creek & WY & 1975 & $1-20$ & 32.5 \\
\hline Lake Ridge & WY & 1981 & 1.20 & 14.0 \\
\hline $\begin{array}{l}\text { Top } 20 \text { Volume Subtoted } \\
\text { Top } 20 \text { Percentage of U.S. Total }\end{array}$ & & & $\begin{array}{r}47,596.9 \\
27.7 \% \\
\end{array}$ & $\begin{array}{r}3,629.5 \\
18.0 \% \\
\end{array}$ \\
\hline Bob Wast & $T x$ & 1900 & $21-50$ & $10 \mathrm{~B} .6$ \\
\hline Anschulz Ranch East & UT \& WY & 1979 & $21-50$ & 79.1 \\
\hline Strong City District & OK & 1986 & $21-50$ & 73.4 \\
\hline Whilney Canyon-Carter Creek & Wh & 1978 & 21.50 & 73.0 \\
\hline Mocane-Laveme Gas Arra & OK \& KS\& DX & 1946 & $21-50$ & 69.6 \\
\hline Mobile B1k 823 & GF & 1983 & $21-50$ & 52.0 \\
\hline Oak Hالثال & $\mathbf{D X}$ & 1958 & $21-50$ & 48.7 \\
\hline Kuista & ок & 1014 & $21-50$ & 46.4 \\
\hline Nelural Bulleg & UT & 1940 & $2\}-50$ & 44.8 \\
\hline Sawyer & $T x$ & 1980 & $21-50$ & 43.1 \\
\hline Nora & VA & 1949 & $21-50$ & 39.8 \\
\hline Lower Moblle Bay-Ntary Ann & AL & 1979 & $21 \cdot 50$ & 36.3 \\
\hline Brady & $w r$ & 1872 & $21-50$ & 35.5 \\
\hline Beluga Aiver & $\mathbf{A K}$ & 1962 & $21-50$ & 34.9 \\
\hline Mobile Bak 864 & GF & 1983 & $21-50$ & 31.4 \\
\hline Mississippl Canyon Blk $73 t$ & GF & 1987 & $21-50$ & 10.8 \\
\hline Judge Dagby & LA & 1977 & 21.50 & 122 \\
\hline Vhosca Knofl Blk 958 & GF & 1995 & $21-50$ & 8.7 \\
\hline Wasson & $\mathrm{TX}$ & 1937 & 21.50 & 2.5 \\
\hline Missiseipgi Canyon Bk 854 & GF & 1992 & 21.50 & 0 \\
\hline East Breaks Blk 945 & GF & 1994 & 21.50 & 0 \\
\hline $\begin{array}{l}\text { Top S0 Volume Subtotal } \\
\text { Top So Puroentagt of U.s. Tolal }\end{array}$ & & & $\begin{array}{r}65,643.7 \\
37.4 \%\end{array}$ & $\begin{array}{r}6,015.2 \\
24,0 \% \\
\end{array}$ \\
\hline
\end{tabular}


Table E2. Top 100 U.S. Fields Ranked by Gas ${ }^{2}$ Production within Proved Reserves Group, from Reported 1997 Field Level Data (Continued) (Bittion Cubic Feat)

\begin{tabular}{|c|c|c|c|c|}
\hline Fleld Name & Locatlon & $\begin{array}{c}\text { Dlacovery } \\
\text { Year }\end{array}$ & $\begin{array}{l}\text { Proved Peservea } \\
\text { Rink Croup }\end{array}$ & $\begin{array}{l}\text { Reportad Protuction } \\
\text { Rank Crous }\end{array}$ \\
\hline Garden Banks BH 426 & GF & 1987 & $51-100$ & 81.5 \\
\hline Matagorda Island Bink be3 & GF & 1980 & $51-100$ & 78.3 \\
\hline Indian Basin & A M & 1903 & 51.100 & 71.7 \\
\hline Mcallen Ranch & $T X$ & 1960 & $51-100$ & 65.1 \\
\hline South Timbalier Eak 17g & GF & 1965 & $51-100$ & 53.6 \\
\hline Jefirests $\mathrm{Ne}$ & $T X$ & 1975 & 51.100 & 52.5 \\
\hline Double A Wells & $T x$ & 1960 & $\$ 1-100$ & \$op. \\
\hline Waltrian & $w r$ & 1959 & $51-100$ & 45.4 \\
\hline Sibley & LA & 1929 & $51-100$ & 45.2 \\
\hline Gkk City & OK & 1947 & $51-100$ & 43.2 \\
\hline Port Hutson & LA & 1977 & $51-100$ & 43.0 \\
\hline South Pass Sa B1k Bs & GF & 1969 & $59-100$ & 42.4 \\
\hline Mississlopl Canyon Blk 354 & GF & 1977 & 51.100 & 40.7 \\
\hline Yiosca Knol 6qk 783 & GF & 1985 & $\$ 1.100$ & 39.8 \\
\hline Boonsuille & $\mathrm{TX}$ & 1045 & $5+100$ & 39.0 \\
\hline Vepden & OK & 1948 & $51-100$ & 38.6 \\
\hline Lake Anther South & LA & 1955 & $51 \cdot 100$ & 36.5 \\
\hline Fainway & AL. & 1886 & $51-100$ & 34.9 \\
\hline Moorawood Ne & $O K$ & 1979 & $5+100$ & 34.5 \\
\hline Mississippi Canyon Blk gob & GF & 1980 & $51-100$ & 33.7 \\
\hline Puckett & $T X$ & 1952 & $51-100$ & 33.5 \\
\hline Brown-Basset1 & $\mathrm{TX}$ & 1993 & $5+100$ & 33.2 \\
\hline Painter Peservoir East & Wr & 1970 & $\$ 1 \cdot 100$ & 32.7 \\
\hline Big Piney & $W Y$ & 1964 & $51-100$ & 边2 \\
\hline Main Pass Blk 41 & GF & $19 \$ 6$ & $51-100$ & 30.9 \\
\hline MFssiosippl Campon Eak 194 & GF & 1975 & $\$ 1-100$ & 29.0 \\
\hline Kupanuk River & AK & 1969 & $51-100$ & 28.5 \\
\hline$A W P$ & $T x$ & 1907 & $51-100$ & 25.6 \\
\hline Newaik East & $T X$ & 1981 & $51-100$ & 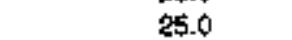 \\
\hline Warnsulter & WY & 1958 & $5 x-100$ & 23.3 \\
\hline Cedar Corve Coal Degas & $A L$ & 1963 & $51 \cdot 100$ & 22.6 \\
\hline Tip Top & $w Y$ & 1928 & $51-100$ & 22.1 \\
\hline Drunkaids Wash & UT & 19甲9g9 & $51-100$ & 21.3 \\
\hline Lost HAlls & $\mathrm{CA}$ & 1910 & $51-100$ & 212 \\
\hline Standard Draw & WY & 1979 & $51-100$ & 20.5 \\
\hline Hondo & PF & 1969 & $51+700$ & 20,4 \\
\hline Trawldk & TX & 1049 & $51-100$ & 19.8 \\
\hline Siratton & TX & 1937 & $51 . t 00$ & 19.0 \\
\hline Pulison & $\infty$ & 1953 & $51-100$ & 16.2 \\
\hline Biance South & NM & 1951 & $51-100$ & 18.0 \\
\hline Belrighe Solth & $\mathrm{CA}$ & 1911 & $51-100$ & 15.3 \\
\hline Sarita East & $\mathbf{T X}$ & 1967 & $51-100$ & 14.9 \\
\hline Kenai & AK & 1059 & $\$ 1-100$ & 12.7 \\
\hline Grand Valley & $\infty$ & 1985 & $51-100$ & 12.3 \\
\hline Sugg Parnch & $7 x$ & 1985 & $51-100$ & 12.3 \\
\hline Endicott & AK & 1978 & $51-100$ & 9.5 \\
\hline Cochrenton & PA & 1980 & $51-100$ & 56 \\
\hline Green Canyon Blk 244 & GF & 1994 & $51-100$ & 1.4 \\
\hline Viosca Know Blk 915 & GF & 1993 & $51 \cdot 100$ & 0.0 \\
\hline Masss|ssippl Camyon E'k 292 & GF & 1900 & $51-100$ & 0.0 \\
\hline \multicolumn{3}{|c|}{ 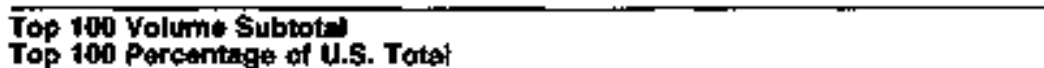 } & $80,698.1$ & 6.562 .7 \\
\hline
\end{tabular}

Awet after lease separation.

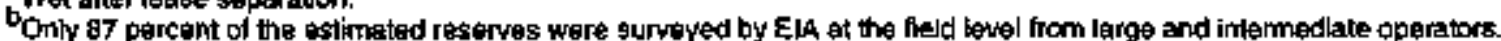

- In Nor Applcable.

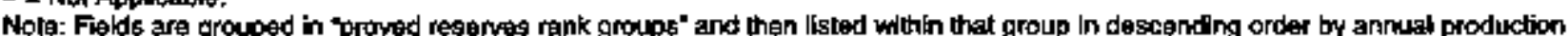
rank. The U.S. Iotal production eslimate of 20,134 bilion cubic leet and the U.S. totad reserves estimate of 175,721 bllion cubic iegt, used to calculate the percenteges in this table, are from Table 9 in this puttication. Column totals may not edd dud to independent rounding.

Source: Enargy Information Adminisiration, Ofiloe of Oll and Ges. 


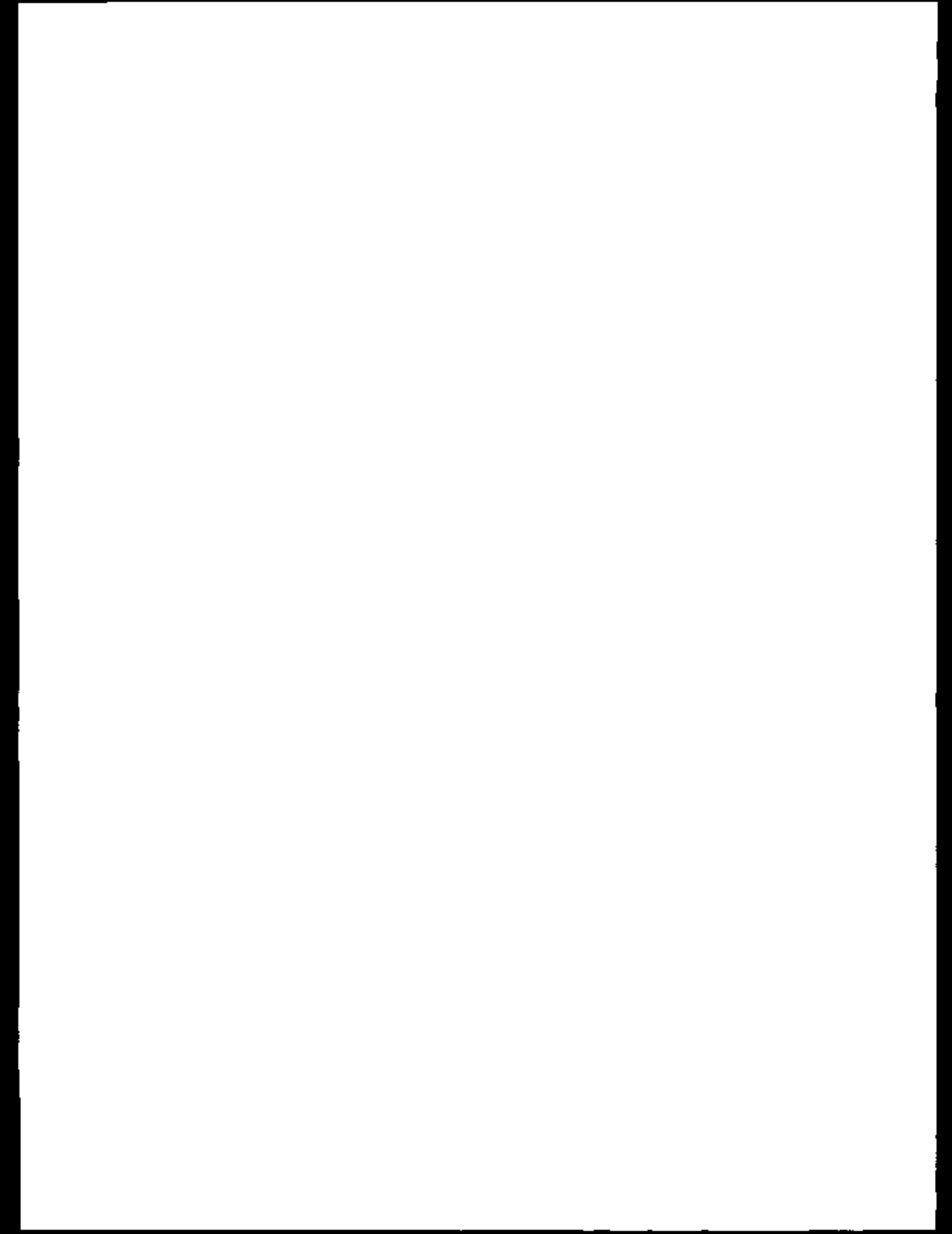


Appendix C

Conversion to the Metric System 



\section{Conversion to the Metric System}

Public Law 100-41B, the Onnibus Trade and Competitiveness Act of 1988, states: "It is the declared policy of the United States-

(1) to designate the metric system of measurement as the preferred system of weights and measures for United States trade and commerce....

(2) to require that each Federal agency, by the end of Fiscal Year 1992, use the metric system of measurement in its procurements, grants, and other business-related activities." $\{40\}$
Table $\mathrm{Cl}$ is in keeping with the spirit of this law. The petroleum industry in the United States is slowly moving in the direction prescribed by this law and the data collected by EJA are collected in the units that are still common to the U.S. petroleurn industry, namely barrels and cubic feet. Standard metric conversion factors were used to convert the Nabional level volumes in Table 1 to the metric equivalents in Table C1. Barrels were multiplied by 0.1589873 to convert to cubic meters and cubic feet were multiplied by 0.02831685 to convert to cubic meters. 
Table C1. U.S. Proved Peserves of Crude Oil, Dry Natural Gas, and Natural Gas Liquids, in Metric Units, 1987 - 1997

\begin{tabular}{|c|c|c|c|c|c|c|c|c|c|c|c|}
\hline Year & Adpustments & 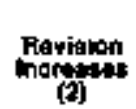 & $\begin{array}{l}\text { Revision } \\
\text { Decruasus } \\
\text { (3) }\end{array}$ & $\begin{array}{l}\text { Poviskons" } \\
\text { and } \\
\text { Adjustmends } \\
\text { [4] }\end{array}$ & $\begin{array}{c}\text { Exiengons } \\
\text { (b) }\end{array}$ & Ching Finkt & 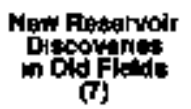 & $\begin{array}{c}\text { Tolal's } \\
\text { Desconertes } \\
\text { (b) }\end{array}$ & $\begin{array}{l}\text { Production } \\
\text { (ब) }\end{array}$ & 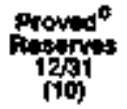 & $\begin{array}{l}\text { Changes } \\
\text { Irom } \\
\text { Prigr } \\
\text { (11) }\end{array}$ \\
\hline
\end{tabular}

Cruade Oil (million cubtc maters)

\begin{tabular}{|c|c|c|c|c|c|c|c|c|c|c|c|}
\hline 1987 & 372 & 5862 & 2180 & 4054 & 769 & 153 & 176 & 1098 & 456 日 & 4,3334 & 584 \\
\hline 1988 & 578 & 4267 & 1941 & 2904 & 564 & 113 & 202 & $\mathbf{B ?}$ & 4469 & 4,2648 & -566 \\
\hline 1969 & 339 & 4209 & 2170 & 2458 & 817 & 178 & 143 & 1138 & 4111 & 4,2133 & -515 \\
\hline 1900 & 137 & 3948 & 1500 & 2465 & 725 & 156 & 215 & 1096 & 3983 & $4.17 \$ 1$ & -392 \\
\hline 1981 & 259 & 3934 & 2979 & 614 & 590 & 154 & 146 & 600 & $\operatorname{sog} 4$ & $3.92 * 1$ & -2500 \\
\hline 1902 & $4 \theta 2$ & 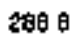 & 1700 & 1 的 0 & 622 & 13 & 135 & 770 & 3889 & 3.7752 & -1489 \\
\hline 1983 & 431 & 3197 & 2410 & 1218 & $\$ 65$ & 607 & 176 & 1248 & 3719 & 3,5498 & -1253 \\
\hline 1094 & 301 & 3758 & 2157 & 1902 & 601 & 102 & 176 & 909 & 3506 & 3,5704 & -795 \\
\hline 1995 & 194 & 2899 & 1264 & 1的 8 & 705 & 181 & 545 & 1521 & 3518 & 3,5535 & $-+B 9$ \\
\hline 190 & 280 & 2739 & 1558 & 1451 & 863 & अ 6 & 224 & 1473 & 3455 & 3,5004 & -531 \\
\hline 1997 & ex 7 & 3177 & 1723 & 2280 & 758 & 2013 & 189 & 1960 & 3399 & 3,5842 & 838 \\
\hline
\end{tabular}

Dry Natural Bas (bulkon cubc meters)

\begin{tabular}{|c|c|c|c|c|c|c|c|c|c|c|c|}
\hline 19a? & 3591 & $40 \leqslant 31$ & 40258 & 12924 & 129 的 & 3084 & 4245 & $\cos 18$ & 45830 & 5,30129 & $-123 \mathrm{AB}$ \\
\hline 1968 & 6200 & 65169 & $d_{1,0}$ & -35436 & 19264 & 4630 & 5406 & 29309 & 47204 & 44,75791 & -54932 \\
\hline 1989 & 8535 & 755 & 68950 & 17119 & 17950 & 4106 & 851 & 284 b7 & 4891 & $4,7 \$ 220$ & -2571 \\
\hline 1990 & 4408 & 53748 & 39068 & 20090 & 22518 & 5675 & 6830 & 35023 & 49 & 4,79535 & 8315 \\
\hline 1991 & 6382 & 56322 & $438+7$ & 20887 & Itad 13 & 2401 & 4542 & 21356 & 48711 & 4,73067 & -8488 \\
\hline 1992 & 6329 & 51126 & 33873 & 23582 & 13238 & 18. 38 & $48 B 2$ & 19858 & 49636 & 467271 & -5796 \\
\hline 1999 & 2751 & 49829 & 34682 & 17898 & 17282 & 2546 & 5284 & 25112 & 50573 & $4,599 \mathrm{nB}$ & $-73 \mathrm{Bg}$ \\
\hline 1994 & 5508 & 60499 & 449 70 & 21037 & 19555 & 5380 & 9854 & $34: 72$ & 51882 & 4,63935 & 4027 \\
\hline 1995 & 1642 & 57950 & 36050 & 23542 & 19377 & 4718 & 6943 & 31039 & 50874 & 4,67841 & 3700 \\
\hline 1990 & 10718 & 48512 & 36942 & 22288 & 21965 & 4109 & 8807 & 34981 & 53408 & 4,71402 & 3781 \\
\hline 1997 & -7570 & 63328 & 47447 & 12710 & 29973 & 7592 & 6745 & $\$ 4310$ & 34400 & 4,73529 & 2122 \\
\hline
\end{tabular}

Matural Gas, Liquits (mmon cubuc meters)

\begin{tabular}{|c|c|c|c|c|c|c|c|c|c|c|c|}
\hline 1987 & 368 & 1347 & 1043 & 672 & 339 & 62 & 67 & $4 B B$ & 1180 & 1,8933 & -28 \\
\hline 1988 & 18 & 1857 & 1137 & 738 & $42 \pi$ & 65 & 114 & 605 & $t 199$ & 13097 & 144 \\
\hline وفئو & -440 & 1817 & 1622 & -245 & 412 & 132 & $11 \mathrm{~B}$ & 的 2 & 1162 & $1.235 \mathrm{z}$ & -745 \\
\hline 1990 & -132 & $13+5$ & 943 & 220 & 475 & 62 & 116 & 653 & 1164 & 1.2051 & $-29 t$ \\
\hline 1991 & 371 & 1312 & 1105 & 578 & 300 & 40 & 87 & 427 & 1199 & 1,1987 & -184 \\
\hline 1992 & $\$ 57$ & 2281 & 的市 & 772 & 해루 & 32 & 102 & 436 & T2t 9 & 1,1845 & -21 \\
\hline 1983 & 162 & 1215 & 1018 & 359 & उष्ष 0 & 38 & $10 \hat{2}$ & 530 & 1253 & 1,1482 & -364 \\
\hline 1984 & 69 & $138 \mathrm{~B}$ & 1075 & 382 & 499 & 86 & $20 \mathrm{~B}$ & 793 & 1258 & 1,1399 & -83 \\
\hline 1998 & 306 & 1639 & 1099 & 745 & 687 & 83 & 107 & $B 77$ & 1258 & 1,1763 & 364 \\
\hline 1996 & 755 & 1342 & 1064 & 1033 & 717 & 103 & 173 & 693 & 1355 & 1,2498 & 675 \\
\hline 1997 & .24 & 1906 & 1447 & 438 & 85 1 & 18t & 14\$ & 1175 & 133 & 1,267 日 & $2 \$ 8$ \\
\hline
\end{tabular}

\footnotetext{
Revisions and adjustrients - Col $1+\operatorname{Col} 2-\operatorname{Cot} 3$

b

CProved reserves - Col 10 hom pror year + Col $4+$ Col B- Col 5

A An unusualy large revision decrease to North Shops dry natural gas reserves was made in 1996 it recogrizes some 69659 billon

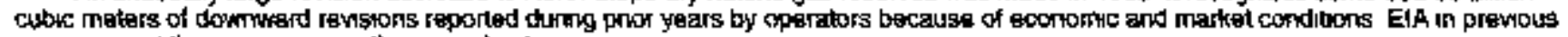
years carriet thesa reserves in the proved calegory

Notes Old means dhecowered in a pnor year New means disco vered dunng the report year The production esturnates in this table are based on deta reported on Form ElA- 23 , "Annupl Survay of Domestoc Gal and Gas Reserves" and Fomm Ela-64A, "Annulyal Fepoorl of the Orkgn of Natural Gas Liquids Producbon " The fothowng conversion fachors were used to convert data m Columns 2, 3, 5, 6, 7, 9, and 10

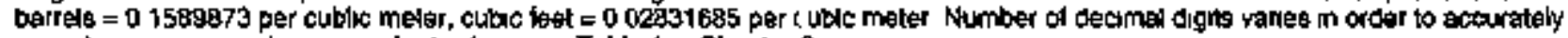
reproduce cortesponding equivalents shiown on Table 1 in Chapter 2

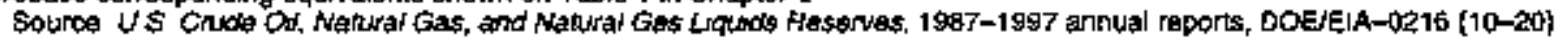


Appendix D

Historical Reserves Statistics 



\section{Historical Reserves Statistics}

These are selected historical data presented at the State and National level. All historical statigticg included have previously been published in the annual reports of 1977 through 1997 of the ELA publication L.S. Crude Oil, Naturat Gas, and Natural Gas Liquids Reserves, DOE ELA-0216-\{1-20\}

Liquid volumes are in million barrels of 42 U.S. gallons. Gas volumes are in billion cubic feet (Bcf), at 14,73 psia and $60^{\circ}$ Fahternheit. NA appears in this appendix wherever data are not available or are withheld to avoid disclosure of data which may be proprietary. An asterisk ( $\left.{ }^{(}\right)$marks those estimates associated with sampling errors (95 percent confidence interval) greater than 20 percent of the value estimated.

\begin{tabular}{|c|c|c|c|c|}
\hline Year & $\begin{array}{l}\text { Crudt Oil } \\
\text { Proved } \\
\text { Reservess }\end{array}$ & $\begin{array}{l}\text { Crude ofi } \\
\text { Indictatod } \\
\text { Adchtlonal } \\
\text { Recerves }\end{array}$ & $\begin{array}{l}\text { Dry } \\
\text { Natural } \\
\text { Gas } \\
\text { Proved } \\
\text { Peserves }\end{array}$ & $\begin{array}{l}\text { Natural } \\
\text { coss } \\
\text { Liqulds } \\
\text { Proved } \\
\text { Peserves }\end{array}$ \\
\hline
\end{tabular}

\begin{tabular}{|c|c|c|c|c|}
\hline Year & $\begin{array}{l}\text { Crude Oil } \\
\text { Proved } \\
\text { Reserves }\end{array}$ & $\begin{array}{l}\text { Crudte oll } \\
\text { Indicaled } \\
\text { Actitional } \\
\text { Peserves }\end{array}$ & $\begin{array}{l}\text { Dry } \\
\text { Nagtural } \\
\text { Gas } \\
\text { Proved } \\
\text { Foserves }\end{array}$ & $\begin{array}{c}\text { Natural } \\
\text { Ges } \\
\text { Lquidd } \\
\text { Proved } \\
\text { Reserves }\end{array}$ \\
\hline
\end{tabular}

\begin{tabular}{|c|c|c|c|c|}
\hline \multicolumn{5}{|c|}{ Alaboma } \\
\hline 1977 & 85 & 0 & 530 & NA \\
\hline 1978 & 74 & 0 & 514 & NA \\
\hline 1979 & 45 & NA & 652 & 213 \\
\hline $19 B D$ & 54 & NA & 636 & 226 \\
\hline 1981 & 55 & NA & 648 & 192 \\
\hline 1982 & 54 & NA & $a_{648}$ & 193 \\
\hline 1983 & 51 & NA & $a_{785}$ & 216 \\
\hline 1984 & "6B & NA & Ag61 & 200 \\
\hline 1985 & 69 & NA & $a_{821}$ & 182 \\
\hline 1986 & 56 & 20 & $b_{951}$ & 177 \\
\hline 1987 & 55 & 20 & $b_{842}$ & 168 \\
\hline 1988 & 54 & 20 & $b_{809}$ & 166 \\
\hline 5989 & 43 & 20 & $b_{B 19}$ & 168 \\
\hline 1990 & 44 & $<1$ & $c_{4,125}$ & 170 \\
\hline 1991 & 43 & $<1$ & $c_{5,414}$ & 145 \\
\hline 1092 & 41 & 0 & $C_{5}, 802$ & 171 \\
\hline 1993 & 41 & 0 & $c_{5,140}$ & 158 \\
\hline 1994 & 44 & $\phi$ & $c_{4,830}$ & 142 \\
\hline 1995 & 43 & 0 & $c_{4,968}$ & 120 \\
\hline 1996 & 45 & 0 & $c_{5,033}$ & 119 \\
\hline 1997 & 47 & 0 & $c_{4,968}$ & 83 \\
\hline
\end{tabular}

\begin{tabular}{|c|c|c|c|c|}
\hline \multicolumn{5}{|c|}{ Alaska } \\
\hline $\begin{array}{l}1977 \\
1978\end{array}$ & $\begin{array}{l}8,413 \\
9,384\end{array}$ & $\begin{array}{l}846 \\
398\end{array}$ & $\begin{array}{l}32,243 \\
32,045\end{array}$ & $\begin{array}{l}\text { NA } \\
\text { NA }\end{array}$ \\
\hline 1979 & $\mathbf{8 , 8 7 5}$ & 398 & 32,259 & 23 \\
\hline 1980 & 8,751 & 0 & 33,382 & 11 \\
\hline 1961 & 8.283 & 0 & 33,037 & 10 \\
\hline 1982 & 7,400 & 60 & $\$ 4,990$ & 9 \\
\hline $19 B 3$ & 7,307 & 576 & 34,286 & $B$ \\
\hline 1984 & 7,563 & 369 & 34,476 & 19 \\
\hline 1985 & 7,056 & 379 & 33,847 & 983 \\
\hline 1986 & 6,875 & 902 & 32,664 & 381 \\
\hline 1987 & 7,378 & 566 & 33,225 & 418 \\
\hline 1988 & 6,959 & $\$ 31$ & 9,07B & 401 \\
\hline 1989 & 6,674 & 750 & 8,939 & 380 \\
\hline 1990 & 6,524 & 969 & 9,300 & 340 \\
\hline 1991 & 6.083 & 1,456 & 9.559 & 360 \\
\hline 1992 & 6,022 & 1,331 & 9,630 & 347 \\
\hline 1993 & 5,775 & 1,161 & 9,907 & a21 \\
\hline 1994 & 5,767 & 1,022 & 9,733 & 301 \\
\hline 1995 & 6,580 & 582 & 9,497 & 306 \\
\hline 1996 & 5,274 & 962 & 9,294 & 337 \\
\hline 1997 & 5,161 & 832 & 10,582 & 631 \\
\hline
\end{tabular}

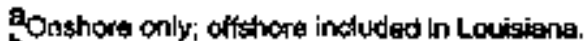

Onshore conly: offshore Inctuded in Federiel Offshore - Gulf of Mhexloo (Louisienta).

Cucludes State ollahore: 2,519 Bct in $1990 ; 3,191$ 8cf in 1991: 3,233 Bcf in 1992: 3,364 Be in 1993; 3,297 Bef in 1994; 3,432 Bef in $1995 ; 3,509$ Bei in 1996; 3,420 Bef in 1997 .

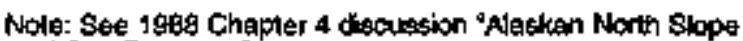
Natural Gas Reservas". 


\begin{tabular}{|c|c|c|c|c|}
\hline Yoar & $\begin{array}{l}\text { Crude ol! } \\
\text { Proved } \\
\text { Pleserves }\end{array}$ & $\begin{array}{l}\text { Crude oil } \\
\text { Indicoted } \\
\text { Addltonal } \\
\text { Reserves }\end{array}$ & $\begin{array}{c}\text { Dry } \\
\text { Malural } \\
\text { Gate } \\
\text { Proved } \\
\text { Pogserves }\end{array}$ & $\begin{array}{l}\text { Natural } \\
\text { Ges } \\
\text { Lquids } \\
\text { Proved } \\
\text { Raserves }\end{array}$ \\
\hline
\end{tabular}

\begin{tabular}{lrrrr}
\hline \multicolumn{5}{c}{ Arkansas } \\
\hline 1977 & 116 & 17 & 1,660 & NA \\
1978 & 111 & 8 & 1,681 & NA \\
1979 & 107 & 6 & 1,703 & 17 \\
1980 & 107 & 11 & 1,774 & 16 \\
1981 & 113 & 11 & 1,801 & 16 \\
1982 & 107 & 4 & 1,958 & 15 \\
1983 & 120 & 4 & 2,069 & 11 \\
1984 & 114 & 6 & 2,227 & 12 \\
1985 & 97 & 11 & 2,019 & 11 \\
1986 & 69 & 9 & 1,992 & 16 \\
1987 & 82 & 0 & 1,997 & 16 \\
1998 & 77 & $<1$ & 1,986 & 13 \\
1989 & 66 & 1 & 1,772 & 9 \\
1990 & 60 & 1 & 1,731 & 9 \\
1991 & 70 & 0 & 1,669 & 5 \\
1992 & 58 & 51 & 1,750 & 4 \\
1993 & 65 & 0 & 1,552 & 4 \\
1994 & 51 & 0 & 1,607 & 6 \\
1995 & 48 & 0 & 1,563 & 6 \\
1996 & 58 & 0 & 1,470 & 4 \\
1997 & 45 & 0 & 1,475 & 7 \\
& & & &
\end{tabular}

\begin{tabular}{|c|c|c|c|c|}
\hline \multicolumn{5}{|c|}{ Caltifornie - Total } \\
\hline $\begin{array}{l}1977 \\
1978 \\
1979 \\
1980 \\
1961 \\
1982 \\
1983 \\
1964 \\
1985 \\
1986 \\
1987 \\
1986 \\
1989 \\
1990 \\
1991 \\
1992 \\
1993 \\
1994 \\
1995 \\
1996 \\
1997\end{array}$ & $\begin{array}{r}5,0,55 \\
4,974 \\
5,265 \\
5,470 \\
5,441 \\
5,405 \\
5,348 \\
5,707 \\
d_{4,810} \\
d_{4,734} \\
d_{4,709} \\
d_{4,879} \\
d_{4,816} \\
d_{4,658} \\
d_{4,217} \\
d_{3,893} \\
d_{3,764} \\
d_{3,573} \\
d_{3,462} \\
d_{3,437} \\
d_{3,750}\end{array}$ & $\begin{array}{r}7,047 \\
968 \\
9900 \\
891 \\
660 \\
616 \\
576 \\
674 \\
590 \\
d_{616} \\
d_{1,493} \\
d_{1,440} \\
d_{1,608} \\
d_{1,425} \\
d_{1,471} \\
d_{1,299} \\
d_{965} \\
d_{635} \\
d_{823} \\
d_{905} \\
d_{1,264}\end{array}$ & $\begin{array}{l}4,737 \\
4,947 \\
5,022 \\
5,414 \\
5,617 \\
5,552 \\
5,781 \\
5,554 \\
d_{4,325} \\
d_{3,928} \\
d_{3,740} \\
d_{3,519} \\
d_{3,374} \\
d_{3,185} \\
d_{3,004} \\
d_{2,779} \\
d_{2,682} \\
d_{2,402} \\
d_{2,243} \\
d_{2,082} \\
d_{2,273}\end{array}$ & $\begin{array}{r}N A \\
N A \\
111 \\
120 \\
82 \\
154 \\
151 \\
d_{146}^{141} \\
d_{134} \\
d_{130} \\
d_{123} \\
d_{113} \\
d_{105} \\
d_{92} \\
d_{99} \\
d_{104} \\
d_{92} \\
d_{92} \\
d_{92} \\
d_{95}\end{array}$ \\
\hline
\end{tabular}

Exchudes Federal ofishore; now inchuded in Federa Offohore. Pacilic (Cealifornia).

\begin{tabular}{|c|c|c|c|c|}
\hline Yoar & $\begin{array}{l}\text { Crude Oil } \\
\text { Proved } \\
\text { Popserves }\end{array}$ & $\begin{array}{l}\text { Grude oil } \\
\text { Inditiated } \\
\text { Addlitonal } \\
\text { Ripserves }\end{array}$ & $\begin{array}{c}\text { Dry } \\
\text { Natural } \\
\text { Cas } \\
\text { Proved } \\
\text { Reserwes }\end{array}$ & $\begin{array}{c}\text { Netural } \\
\text { Gass } \\
\text { Liquids } \\
\text { Proved } \\
\text { Peserves }\end{array}$ \\
\hline
\end{tabular}

\begin{tabular}{lcccc}
\hline \multicolumn{5}{c}{ Calfornia - Coastal Feglon Onshore } \\
\hline 1977 & 679 & NA & 334 & NA \\
1978 & 602 & NA & 350 & NA \\
1979 & 578 & NA & 365 & 22 \\
1980 & 652 & NA & 299 & 23 \\
1981 & 621 & NA & 306 & 14 \\
1982 & 580 & NA & 362 & 16 \\
1983 & 659 & NA & 381 & 17 \\
1984 & 628 & 140 & 265 & 15 \\
1985 & 631 & 152 & 256 & 16 \\
1986 & 592 & 164 & 255 & 15 \\
1987 & 625 & 298 & 239 & 13 \\
1989 & 576 & 299 & 215 & 13 \\
1989 & 731 & 361 & 224 & 11 \\
1990 & 589 & 310 & 217 & 12 \\
1991 & 554 & 327 & 216 & 12 \\
1992 & 522 & 317 & 203 & 10 \\
1993 & 528 & 313 & 189 & 12 \\
1994 & 480 & 238 & 194 & 11 \\
1995 & 456 & 234 & 153 & 8 \\
1996 & 425 & 261 & 156 & 9 \\
1997 & 430 & 43 & 164 & 9
\end{tabular}

\begin{tabular}{|c|c|c|c|c|}
\hline \multicolumn{5}{|c|}{ Califomla - Los Angeles Basin Onshore } \\
\hline 1977 & 910 & NA & 255 & NA \\
\hline 1978 & 493 & NA & 178 & NA \\
\hline 1979 & 513 & NA & 160 & 10 \\
\hline 1980 & 454 & NA & 190 & $\uparrow 5$ \\
\hline 1981 & 412 & NA & 154 & 6 \\
\hline 1982 & 370 & NA & 90 & $\mathbf{6}$ \\
\hline 1993 & 343 & NA & 107 & 6 \\
\hline 1984 & 373 & 126 & 156 & $\mathbf{5}$ \\
\hline 1986 & 420 & 86 & 181 & 6 \\
\hline 1986 & 330 & 66 & 142 & 8 \\
\hline 1987 & 安1 & 105 & 148 & 8 \\
\hline 1988 & 391 & 106 & 151 & 7 \\
\hline 1989 & 342 & 32 & 137 & 4 \\
\hline 1990 & 316 & 3 & 100 & 5 \\
\hline 1991 & 272 & 4 & 115 & 4 \\
\hline 1992 & 236 & 4 & 97 & 6 \\
\hline 1993 & 238 & 4 & 102 & 6 \\
\hline 1994 & 221 & 4 & 109 & 5 \\
\hline 1995 & 227 & 4 & 111 & 4 \\
\hline 1986 & 234 & 0 & 109 & 3 \\
\hline 1997 & 268 & 0 & 141 & 4 \\
\hline
\end{tabular}



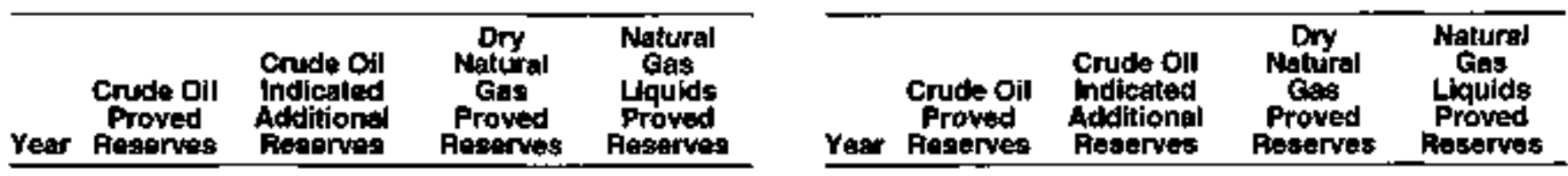

\begin{tabular}{|c|c|c|c|c|c|c|c|c|c|}
\hline \multicolumn{5}{|c|}{ Caltifornia - San Joaquin Basin Onshore } & \multicolumn{5}{|c|}{ California - State Offare } \\
\hline 1977 & 2,965 & NA & 3,784 & NA & 1977 & 181 & NA & 114 & NA \\
\hline 1978 & 3,090 & NA & 3,940 & NA & +978 & $\$ 19$ & NA & 213 & NA \\
\hline 1979 & 3,294 & NA & 3,941 & 77 & 1979 & 692 & NA & 231 & 2 \\
\hline 1980 & 3,360 & NA & 4,344 & 81 & $\dagger 9 \mathrm{gO}$ & 604 & NA & 164 & 1 \\
\hline 1981 & 3,225 & NA & 4,163 & 57 & 1981 & $\mathrm{NA}$ & NA & NA & NA \\
\hline 1982 & $3,0 \mathrm{B1}$ & 神A & 3901 & 124 & 1982 & NA & $\mathrm{NA}$ & NA & NA \\
\hline 1983 & 3,032 & NA & 3,819 & 117 & 1993 & NA & NA & NA & NA \\
\hline 1984 & 3,197 & 384 & 3,685 & 105 & 1984 & NA & 25 & NA & NA \\
\hline 1985 & 3,250 & 350 & 3,574 & 120 & 1985 & 501 & 0 & $\$ 14$ & 4 \\
\hline 1986 & 3,270 & 368 & 3,277 & 109 & 1986 & 542 & 18 & 254 & 2 \\
\hline 1987 & 3,208 & 1,070 & 3,102 & 107 & 1997 & 515 & 18 & 252 & 2 \\
\hline 1988 & 3,439 & 1,029 & 2,912 & 101 & 1988 & 473 & 6 & 241 & 2 \\
\hline 1989 & 3,301 & 1,210 & $2,7 \mathrm{B2}$ & 95 & 1989 & 442 & 5 & 231 & 3 \\
\hline 1990 & 3,334 & 1,109 & 2,670 & 66 & 1990 & 420 & 3 & 192 & 2 \\
\hline 1991 & 3.126 & 1,139 & 2,614 & 75 & 1991 & 265 & 1 & 59 & 1 \\
\hline 1992 & 2,898 & 977 & 2,415 & 83 & 1992 & 237 & 1 & 63 & 1 \\
\hline 1993 & 2,772 & 648 & 2,327 & 85 & 1993 & 226 & 0 & 64 & $t$ \\
\hline 1994 & 2,647 & 593 & 2,044 & 75 & 1094 & 225 & 0 & 61 & 1 \\
\hline 1905 & 2,577 & 586 & 1,920 & 80 & 1995 & 202 & 0 & 59 & 0 \\
\hline 1996 & 2,597 & 644 & 1,768 & 80 & 1996 & 181 & 0 & 49 & 0 \\
\hline 1997 & 2,871 & 1,221 & 1,912 & 62 & 1997 & 181 & 0 & 56 & 0 \\
\hline
\end{tabular}

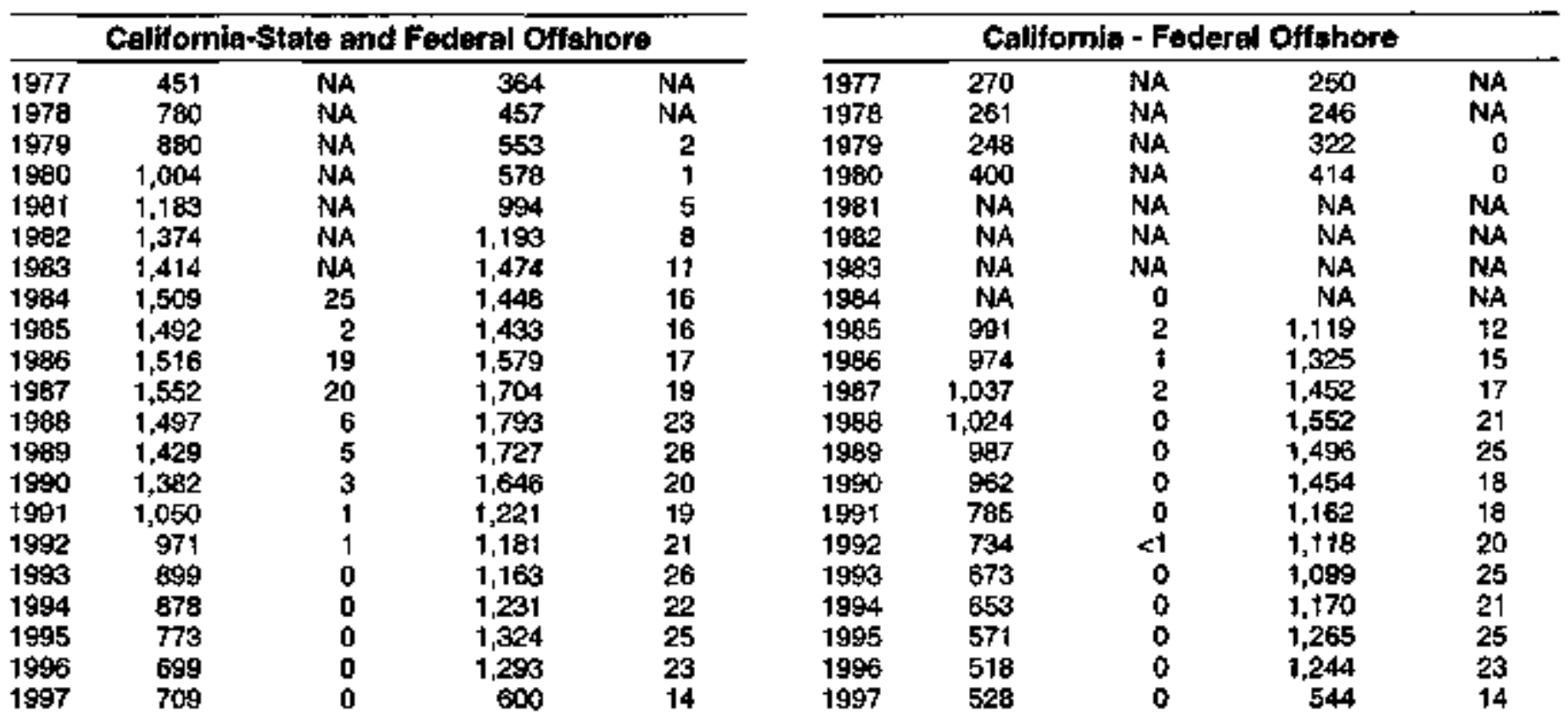




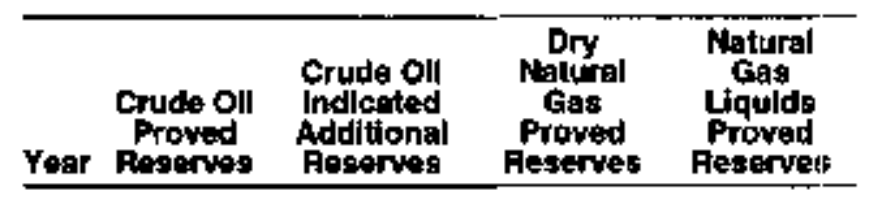

\begin{tabular}{|c|c|c|c|c|}
\hline Year & $\begin{array}{l}\text { Crudde Ojl } \\
\text { Proved } \\
\text { Ripserves }\end{array}$ & $\begin{array}{l}\text { Crude Oit } \\
\text { Indicated } \\
\text { Additionel } \\
\text { Retserves }\end{array}$ & $\begin{array}{c}\text { Dry } \\
\text { Natural } \\
\text { Gas } \\
\text { Proved } \\
\text { Reserves }\end{array}$ & $\begin{array}{l}\text { Natural } \\
\text { Gap } \\
\text { Liquids } \\
\text { Proved } \\
\text { Reserves }\end{array}$ \\
\hline
\end{tabular}

\begin{tabular}{lrrrr}
\hline \multicolumn{5}{c}{ Colorado } \\
\hline 1977 & 290 & 73 & 2,512 & NA \\
1978 & 194 & 75 & 2,765 & NA \\
1979 & 159 & 43 & 2,608 & 177 \\
1980 & 189 & 46 & 2,922 & 194 \\
1981 & 147 & 47 & 2,961 & 204 \\
1982 & 169 & 100 & 3,314 & 186 \\
1983 & 186 & 113 & 3,148 & 183 \\
1984 & 198 & 119 & 2,943 & 155 \\
1985 & 198 & 119 & 2,881 & 173 \\
1986 & 207 & 95 & 3,027 & 148 \\
1987 & 272 & 67 & 2,942 & 165 \\
1988 & 257 & 67 & 3,535 & 181 \\
1999 & 359 & 8 & 4,274 & 209 \\
1990 & 305 & 8 & 4,555 & 169 \\
1991 & 329 & 33 & 5,767 & 197 \\
1992 & 304 & 34 & 6,198 & 226 \\
1993 & 284 & 22 & 6,722 & 214 \\
1994 & 271 & 22 & 6,753 & 248 \\
1995 & 252 & 24 & 7,256 & 273 \\
1996 & 231 & 22 & 7,710 & 287 \\
1997 & 196 & 22 & 6,826 & 264
\end{tabular}

\begin{tabular}{|c|c|c|c|c|}
\hline \multicolumn{5}{|c|}{ Illinois } \\
\hline 1977 & .150 & 1 & NA & NA \\
\hline 1978 & +158 & 1 & NA. & NA \\
\hline 1979 & ×1\$6 & 1 & NA & $N A$ \\
\hline 1880 & 113 & 2 & NA & NA \\
\hline 1981 & 129 & 1 & NA & NA \\
\hline 1982 & 150 & 1 & NA & NA \\
\hline 1983 & 135 & 1 & NA & NA \\
\hline 1984 & 153 & 1 & NA & NA \\
\hline 1985 & 136 & 1 & NA & NA \\
\hline 1986 & 135 & 1 & NA & NA \\
\hline 1987 & 153 & 5 & NA & NA \\
\hline 1988 & 143 & $<1$ & NA & NA \\
\hline 1989 & 123 & $<1$ & NA & $\mathrm{NA}$ \\
\hline 1 망이 & 131 & 0 & NA & $\mathrm{NA}$ \\
\hline 1991 & 128 & 52 & NA & $\mathrm{NA}$ \\
\hline 1992 & 138 & 0 & NA & NA \\
\hline 1993 & 116 & 0 & NA & NA \\
\hline 1994 & 117 & 0 & NA & NA \\
\hline 1095 & 119 & 0 & NA & $\mathrm{NA}$ \\
\hline 1996 & 94 & 0 & NA & $\mathrm{NA}$ \\
\hline 1997 & 92 & 0 & NA & NA \\
\hline
\end{tabular}

\begin{tabular}{lcccc}
\hline & \multicolumn{3}{c}{ Florida } & \\
\hline 1977 & 213 & 1 & 151 & NA \\
1978 & 168 & 1 & 119 & NA \\
1979 & 128 & 1 & 77 & 21 \\
1980 & 134 & 1 & 84 & 27 \\
1981 & 109 & 1 & 69 & NA \\
1982 & 97 & 1 & 64 & 17 \\
1983 & 76 & 4 & 49 & 11 \\
1984 & 82 & 2 & 65 & 17 \\
1965 & 77 & 2 & 55 & 17 \\
1986 & 67 & 2 & 49 & 14 \\
1987 & 61 & 0 & 49 & 9 \\
1988 & 59 & 0 & 51 & 16 \\
1989 & 60 & 0 & 46 & 10 \\
1990 & 42 & 0 & 45 & B \\
1991 & 37 & 0 & 38 & 7 \\
1992 & 36 & 0 & 47 & 9 \\
1993 & 40 & 0 & 50 & 9 \\
1994 & 71 & 0 & 98 & 18 \\
1995 & 71 & 0 & 92 & 17 \\
1996 & 97 & 0 & 96 & 22 \\
1997 & 91 & 0 & 96 & 17
\end{tabular}

\begin{tabular}{|c|c|c|c|c|}
\hline \multicolumn{5}{|c|}{ Indtiana } \\
\hline 1977 & 20 & 0 & NA & NA \\
\hline 1970 & 29 & 0 & $\mathrm{MAA}$ & NA \\
\hline 1979 & 40 & 0 & $N A$ & NA \\
\hline 1980 & 23 & 0 & NA & NA \\
\hline 1881 & 23 & 0 & NA & NA \\
\hline 1982 & 28 & 1 & NA & NA \\
\hline 1983 & 34 & 3 & NA & NA \\
\hline 1984 & 133 & 2 & NA & NA \\
\hline 1905 & 35 & 2 & NA & NA \\
\hline 1986 & +32 & 2 & NA & NA \\
\hline 1987 & 23 & 2 & NA & NA \\
\hline 1988 & $\cdot 22$ & 0 & NA & NA \\
\hline 1989 & +16 & 0 & NA & NA \\
\hline 1990 & 12 & 0 & NA & NA \\
\hline 1991 & +16 & 0 & NA & NA \\
\hline 1992 & 17 & 0 & NA & NA \\
\hline 1998 & 15 & 0 & NA & NA \\
\hline 1994 & 15 & 0 & NA & NA \\
\hline 1995 & 13 & 0 & NA & NA \\
\hline 1996 & 11 & 0 & NA & NA \\
\hline 1997 & +10 & 0 & NA & NA \\
\hline
\end{tabular}




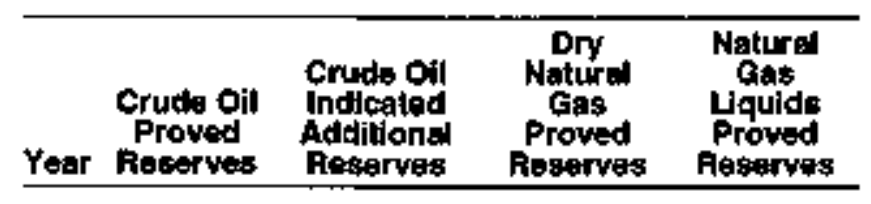

\begin{tabular}{|c|c|c|c|c|}
\hline Yoar & $\begin{array}{l}\text { Crube oll } \\
\text { Proved } \\
\text { freserves }\end{array}$ & $\begin{array}{l}\text { Cride oll } \\
\text { Indicaled } \\
\text { Addillonel } \\
\text { Reserves }\end{array}$ & $\begin{array}{l}\text { Dry } \\
\text { Natural } \\
\text { Cas } \\
\text { Proved } \\
\text { Reserves }\end{array}$ & $\begin{array}{l}\text { Natured } \\
\text { Ges } \\
\text { Liquids } \\
\text { Proved } \\
\text { Pogerves }\end{array}$ \\
\hline
\end{tabular}

\begin{tabular}{lrrrr}
\hline \multicolumn{5}{c}{ Kanstas } \\
\hline 1977 & 349 & 3 & 11,457 & NA \\
1978 & 303 & 3 & 10,992 & NA \\
1979 & -377 & 9 & 10,249 & 402 \\
1990 & 310 & 2 & 9,508 & 389 \\
1981 & 371 & 2 & 9,860 & 409 \\
1992 & 378 & 13 & 9,724 & 302 \\
1983 & 344 & 13 & 9,553 & 443 \\
1994 & 377 & 2 & 9,367 & 424 \\
1995 & 423 & $<1$ & 9,337 & 373 \\
1996 & 312 & $<1$ & 10,509 & 440 \\
1997 & 357 & $<1$ & 10,494 & 462 \\
1998 & 327 & $<1$ & 10,104 & 345 \\
1989 & 338 & 3 & 10,091 & 329 \\
1990 & 321 & $<1$ & 9,614 & 313 \\
1991 & 300 & $<1$ & 9,369 & 428 \\
1992 & 310 & 0 & 9,681 & 444 \\
1993 & 271 & 0 & 9,349 & 380 \\
1994 & 260 & 0 & 9,156 & 399 \\
1995 & 275 & $<1$ & 8,571 & 369 \\
1996 & 266 & $<1$ & 7,694 & 338 \\
1997 & 298 & 0 & 6,989 & 271
\end{tabular}

\begin{tabular}{|c|c|c|c|c|}
\hline \multicolumn{5}{|c|}{ Louislana - Toted } \\
\hline $\begin{array}{l}1977 \\
1978\end{array}$ & $\begin{array}{l}3,600 \\
3.448\end{array}$ & $\begin{array}{l}139 \\
143\end{array}$ & $\begin{array}{l}57.010 \\
55.725\end{array}$ & $\begin{array}{l}N A \\
N A\end{array}$ \\
\hline 1979 & 2,760 & 76 & 50,042 & 1,424 \\
\hline 1980 & 2,751 & 62 & 47,325 & 1,346 \\
\hline 1981 & 2,985 & 50 & 47,377 & 1,327 \\
\hline 1982 & 2,728 & 49 & 944,916 & 1,296 \\
\hline 1983 & 2,707 & 45 & $e_{42,561}$ & 1,332 \\
\hline 1984 & 2,661 & 65 & $\mathrm{e}_{41,399}$ & 1,188 \\
\hline 1985 & 883 & 35 & 14,038 & 546 \\
\hline 1986 & 1826 & 47 & 12,930 & $f_{524}$ \\
\hline 1987 & $f_{807}$ & 155 & $f_{12,430}$ & $f_{525}$ \\
\hline 1988 & $f_{800}$ & $t_{69}$ & $f_{12,224}$ & $f_{517}$ \\
\hline 1989 & $f_{745}$ & $f_{63}$ & 12,516 & 522 \\
\hline 1990 & 1705 & 12 & 111,720 & $t_{538}$ \\
\hline 1991 & 1679 & & $f_{10,912}$ & 526 \\
\hline 1992 & 668 & & 9,780 & t495 \\
\hline 1993 & 1639 & f336 & 19,174 & 421 \\
\hline 1994 & $f_{649}$ & 340 & 9,748 & 4,434 \\
\hline 1995 & 637 & 475 & 9.274 & $f_{601}$ \\
\hline 1996 & 658 & 331 & 9,543 & 1543 \\
\hline 1997 & 1714 & $\mathrm{f}_{313}$ & $f_{9,673}$ & $1_{437}$ \\
\hline
\end{tabular}

Oinchudes State and Faderal olishore Alabama.

Excludes Federal offschore: now incuhded in Federal Ofishore Gulf of Maxich (Louigienta).

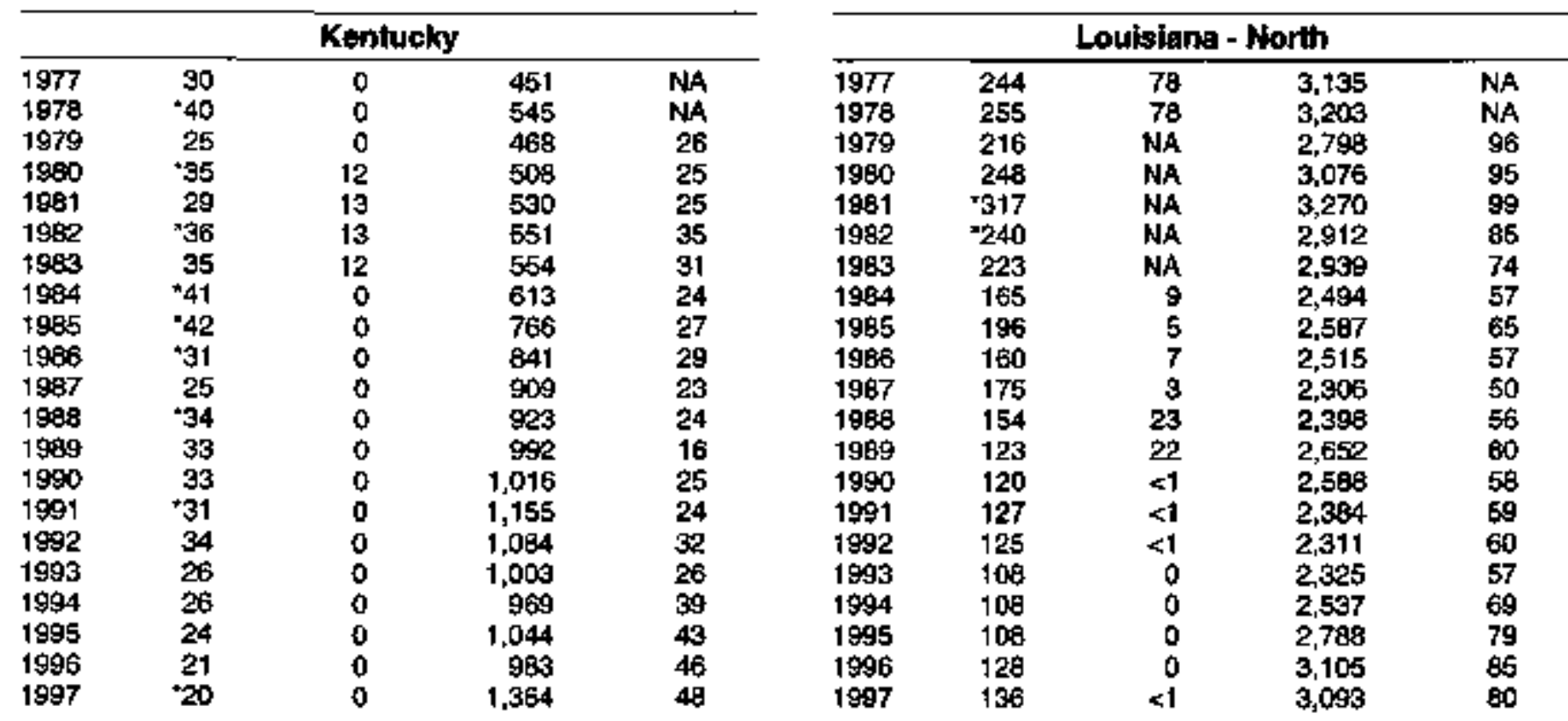




\begin{tabular}{|c|c|c|c|c|}
\hline Yed & $\begin{array}{l}\text { Crude Oil } \\
\text { Proved } \\
\text { Feservits }\end{array}$ & $\begin{array}{l}\text { Crude oll } \\
\text { Indicatod } \\
\text { Additiondat } \\
\text { Rererves }\end{array}$ & $\begin{array}{c}\text { Dry } \\
\text { Natural } \\
\text { Gac } \\
\text { Proved } \\
\text { Reserves }\end{array}$ & $\begin{array}{l}\text { Natural } \\
\text { Gas } \\
\text { Utquids } \\
\text { Proved } \\
\text { Peserved }\end{array}$ \\
\hline
\end{tabular}

\begin{tabular}{|c|c|c|c|c|}
\hline Year & $\begin{array}{l}\text { Crude of } \\
\text { Proved } \\
\text { Pesterves }\end{array}$ & $\begin{array}{l}\text { Crude ôl } \\
\text { Indicitod } \\
\text { Additiondal } \\
\text { Retserves }\end{array}$ & $\begin{array}{l}\text { Dry } \\
\text { Natural } \\
\text { Gas } \\
\text { Proved } \\
\text { Reserves }\end{array}$ & $\begin{array}{l}\text { Malural } \\
\text { Gas } \\
\text { Liquids } \\
\text { Proved } \\
\text { Reserves }\end{array}$ \\
\hline
\end{tabular}

\begin{tabular}{|c|c|c|c|c|}
\hline \multicolumn{5}{|c|}{ Lotisiana - South Onshore } \\
\hline 1977 & 1,3这 & 46 & 18,580 & NA \\
\hline 1978 & 1,242 & 38 & 17,755 & NA \\
\hline 1979 & 682 & NA & 13,994 & 676 \\
\hline 1900 & 682 & NA & 13,026 & 540 \\
\hline 1981 & 642 & NA & 12,645 & 544 \\
\hline 1982 & 611 & NA & 11,801 & 501 \\
\hline 1983 & 569 & NA & 11,142 & 527 \\
\hline 1984 & 585 & 20 & 10,331 & 454 \\
\hline 1985 & 565 & 16 & 9,809 & 442 \\
\hline 1986 & 647 & 30 & 9,10 & 428 \\
\hline 1987 & 505 & 22 & 6,693 & 429 \\
\hline 1988 & 511 & 35 & 8,654 & 421 \\
\hline 1989 & 479 & 30 & 8,645 & 411 \\
\hline 1990 & 435 & 11 & 6,171 & 431 \\
\hline 1991 & 408 & 33 & 7,604 & 417 \\
\hline 1992 & 417 & 26 & 6,693 & 380 \\
\hline 1990 & 382 & 329 & 5,932 & 334 \\
\hline 1994 & - 391 & 331 & 6,251 & 337 \\
\hline 1995 & 397 & 324 & 5,648 & 495 \\
\hline 1990 & 382 & 322 & 5.704 & 411 \\
\hline 1997 & 427 & 309 & 5,855 & 333 \\
\hline
\end{tabular}

\begin{tabular}{|c|c|c|c|c|}
\hline \multicolumn{5}{|c|}{ Michigan } \\
\hline 1977 & 233 & 0 & 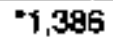 & NA \\
\hline 1978 & $\pi / 220$ & 9 & $* 1,422$ & NA \\
\hline 1979 & 159 & 23 & 1,204 & 112 \\
\hline 1980 & 205 & 14 & $-1,406$ & 112 \\
\hline 1981 & -240 & 17 & 1,178 & 102 \\
\hline 1982 & 184 & 34 & 1,084 & 97 \\
\hline 1963 & 209 & 48 & 1,219 & 105 \\
\hline 1984 & 180 & 46 & 1,112 & 84 \\
\hline 1985 & 191 & 37 & 985 & 67 \\
\hline 1986 & 146 & 94 & 1,139 & 89 \\
\hline 1967 & 151 & 27 & 1,451 & 111 \\
\hline 1988 & 132 & 27 & 1,323 & 99 \\
\hline 1989 & 128 & 8 & 1,342 & 97 \\
\hline 1990 & 124 & 3 & 1,243 & 81 \\
\hline 1991 & 119 & 0 & 1,334 & 72 \\
\hline 1992 & 102 & 0 & 1,223 & 68 \\
\hline 1993 & 90 & 0 & 1,160 & 57 \\
\hline 1994 & 91 & 1 & 1,323 & 54 \\
\hline 1995 & 75 & 1 & 1,294 & 45 \\
\hline 1996 & 74 & 0 & 2,061 & 53 \\
\hline 1997 & 68 & 2 & 2,195 & 50 \\
\hline
\end{tabular}

\begin{tabular}{|c|c|c|c|c|c|c|c|c|c|}
\hline \multicolumn{5}{|c|}{ Loufsiana - Stale Offshort } & \multicolumn{5}{|c|}{ Afrobissippi } \\
\hline 1977 & 1,974 & 15 & 35,295 & NA & 1977 & 241 & 9 & 1,437 & $\mathrm{NA}$ \\
\hline 1978 & 1,951 & 27 & 34,767 & NA & 1978 & 250 & 27 & 1,635 & $\mathrm{NA}$ \\
\hline 1979 & $1,8 \mathrm{Bg}$ & 14 & 33,250 & 652 & 1979 & 238 & 24 & 1,504 & 16 \\
\hline 1980 & 1,821 & 13 & 31,223 & 711 & 1980 & 202 & 36 & 1,769 & 20 \\
\hline 1981 & 2,026 & 16 & 31,462 & 684 & 1981 & 209 & 93 & 2,035 & 18 \\
\hline 1982 & 1,877 & 21 & $\theta_{30,203}$ & 709 & 1982 & 223 & 86 & 1,796 & 18 \\
\hline 1983 & 1,915 & t5 & $\theta_{28,4 B O}$ & 731 & 1983 & 205 & 77 & 1,596 & 19 \\
\hline 1964 & 1,911 & 27 & 28,574 & 677 & 1984 & 201 & 50 & 1,491 & 15 \\
\hline 1985 & 122 & 2 & 1,643 & 30 & 1985 & 184 & 53 & 1,360 & 12 \\
\hline 1986 & $\$ 119$ & & 1,312 & 30 & 1986 & 199 & 16 & 1,300 & 11 \\
\hline 1987 & 1127 & $f_{22}$ & $f, 4,431$ & $1_{46}$ & 1987 & 202 & 12 & 1,200 & 11 \\
\hline 1988 & 1135 & $f_{11}$ & $f_{1,172}$ & 140 & 1988 & 21 & 10 & 1,143 & 12 \\
\hline 1969 & $t 143$ & $f_{11}$ & $f_{1,219}$ & 51 & 1989 & 218 & 6 & 1,104 & 12 \\
\hline 1990 & $f_{150}$ & & & 49 & 1990 & 227 & 8 & 1,126 & 11 \\
\hline 1991 & $t_{144}$ & & $f_{1}, 024$ & 50 & 1991 & 194 & 8 & 1,057 & 10 \\
\hline 1992 & $1 / 26$ & & 776 & 55 & 1992 & 165 & 7 & 869 & 9 \\
\hline 1993 & $f_{149}$ & & $f_{917}$ & 130 & 1903 & 133 & 44 & 797 & 11 \\
\hline 1994 & 150 & & $f_{960}$ & 28 & 1994 & 151 & 40 & 650 & $g$ \\
\hline 1995 & $t_{142}$ & $f_{161}$ & gass & 127 & 1995 & 140 & 6 & 669 & $g$ \\
\hline 1996 & 148 & 9 & 734 & 47 & 1996 & 164 & 6 & 631 & 7 \\
\hline & & & 725 & 124 & 1997 & 183 & 0 & 582 & $\theta$ \\
\hline
\end{tabular}

Oinchudes State ard Federal offshore Alabama.

Exchudes Federal oftshore; now inchuded in Federel Offshore. Butf of Mexico (Lotolgana). 


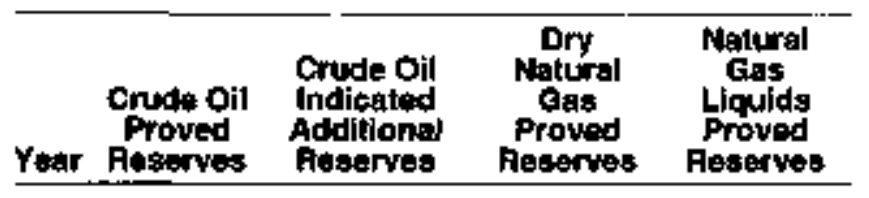

\begin{tabular}{|c|c|c|c|c|}
\hline Year & $\begin{array}{l}\text { Crude Qil } \\
\text { Proved } \\
\text { Peserves }\end{array}$ & $\begin{array}{l}\text { Crude oil } \\
\text { Indicated } \\
\text { Add'tions } \\
\text { Aeserves }\end{array}$ & $\begin{array}{c}\text { Dry } \\
\text { Niatural } \\
\text { Gas } \\
\text { Proved } \\
\text { Aegervas }\end{array}$ & $\begin{array}{l}\text { Natural } \\
\text { Gus } \\
\text { Licuids } \\
\text { Proved } \\
\text { Aeserves }\end{array}$ \\
\hline
\end{tabular}

\begin{tabular}{|c|c|c|c|c|c|c|c|c|c|}
\hline \multicolumn{5}{|c|}{ Montana } & \multicolumn{5}{|c|}{ New Mexico - Total } \\
\hline 1977 & 175 & 27 & '887 & NA & 1977 & 605 & 97 & 12,000 & NA \\
\hline 1978 & 158 & 27 & 926 & NA & 1978 & 579 & 90 & 12,688 & NA \\
\hline 1979 & 152 & 38 & 625 & 10 & 1979 & 563 & 77 & 13,724 & 530 \\
\hline 1980 & 179 & 13 & $-1,287$ & 16 & 1980 & 547 & 58 & 13,297 & 541 \\
\hline 1981 & 186 & 11 & $* 1,321$ & 11 & 1981 & 555 & 90 & 13,870 & 560 \\
\hline 1982 & 216 & 6 & 847 & 18 & 1982 & 583 & 76 & 12,418 & $\mathbf{5 3 1}$ \\
\hline 1983 & 234 & 8 & 896 & 19 & 1983 & 576 & 75 & 11,676 & 551 \\
\hline 1984 & 224 & 4 & 802 & 18 & 1984 & 660 & 87 & $\$ 1,364$ & 511 \\
\hline 1985 & 232 & 3 & 857 & 21 & 1985 & 639 & 99 & 10,900 & 445 \\
\hline 1986 & 248 & 27 & 803 & 16 & 1986 & 644 & 225 & 11,800 & 577 \\
\hline 1987 & 246 & $<1$ & 790 & 16 & 1987 & 654 & 235 & 11,620 & 771 \\
\hline $198 B$ & 241 & 0 & 819 & 11 & 1988 & 661 & 241 & 17,166 & 1,023 \\
\hline 1989 & 225 & $<1$ & 867 & 16 & 1989 & 665 & 256 & 15,434 & 933 \\
\hline 1990 & 221 & 0 & 899 & 15 & 1990 & 687 & 256 & 17,260 & 990 \\
\hline 1991 & 201 & 0 & 831 & 14 & 1991 & 721 & 275 & 18,539 & 908 \\
\hline 1992 & 193 & 0 & 859 & 12 & 1992 & 757 & 293 & 18,998 & 1,066 \\
\hline 1993 & 171 & 0 & 673 & 8 & 1993 & 707 & $2+1$ & 18,619 & 996 \\
\hline 1994 & 175 & 0 & 717 & $\boldsymbol{B}$ & 1994 & 718 & 215 & 17,228 & 1,011 \\
\hline 1995 & 178 & 0 & 782 & $B$ & 1995 & 732 & 185 & 17,491 & 943 \\
\hline 1996 & 168 & 0 & 796 & 7 & 1996 & 744 & 148 & 16,465 & 1,059 \\
\hline 1997 & 159 & 1 & 762 & 5 & 1997 & 735 & 146 & 15,514 & 860 \\
\hline
\end{tabular}

\begin{tabular}{|c|c|c|c|c|c|c|c|c|c|}
\hline \multicolumn{5}{|c|}{ Nebraska } & \multicolumn{5}{|c|}{ Naw Maxico - East } \\
\hline 1977 & 22 & 0 & NA & NA & 1977 & 576 & 95 & 3,848 & NA \\
\hline 1978 & 30 & $t$ & NA & NA & 1978 & 554 & 68 & 3,869 & NA \\
\hline 1979 & 25 & 0 & NA & NA & 1979 & 542 & 77 & 4,031 & 209 \\
\hline 1990 & -46 & 0 & NA & NA & 1980 & 518 & 58 & 3,530 & 209 \\
\hline $198 t$ & $4 t$ & 0 & $\mathrm{NA}$ & NA & 1981 & 522 & 93 & 3,508 & 214 \\
\hline 1982 & -32 & 0 & NA & NA & 1982 & 537 & 76 & $3,4,52$ & 209 \\
\hline 1983 & 44 & 0 & NA & NA & 1963 & 542 & 75 & 3,230 & 232 \\
\hline 1084 & $\star 46$ & 0 & NA & NA & 1984 & 625 & 87 & 3,197 & 221 \\
\hline 1985 & 42 & 0 & NA & $N A$ & 1985 & 649 & 98 & $3,0 \$ 4$ & 209 \\
\hline 1986 & $* 45$ & 7 & NA & NA & 1986 & 593 & 225 & 2,694 & 217 \\
\hline 1987 & 33 & 0 & $N A$ & NA & 1987 & 608 & 230 & 2,881 & 192 \\
\hline $198 B$ & 42 & 0 & NA & NA & 1988 & 621 & 236 & 2,945 & 208 \\
\hline 1989 & 32 & 0 & NA & NA & 1989 & 619 & 252 & 3,075 & 196 \\
\hline 1990 & 26 & 0 & NA & $N A$ & 1990 & 633 & 253 & 3,256 & 222 \\
\hline 1991 & 26 & 0 & NA & NA & 1991 & 694 & 275 & 3,206 & 205 \\
\hline 1992 & 26 & 0 & NA & MA & 1992 & 731 & 293 & 3,130 & 223 \\
\hline 1993 & 20 & 0 & NA & NA & $199 \mathrm{~s}$ & 688 & 211 & 3,004 & 233 \\
\hline 1894 & 22 & 0 & NA & NA & 1994 & 702 & 215 & 3,021 & 234 \\
\hline 1995 & 25 & 0 & NA & NA & 1995 & 713 & 185 & 2,867 & 247 \\
\hline 1996 & 28 & 0 & NA & NA & 1996 & 731 & 148 & 2,700 & 299 \\
\hline 1997 & -21 & 0 & NA & NA & 1997 & 719 & 146 & 2,642 & 273 \\
\hline
\end{tabular}

Eneroy Informotion Adminletrition 


\begin{tabular}{cccc}
\hline Crude Oll & $\begin{array}{c}\text { Crude oil } \\
\text { Indlceted } \\
\text { Proved } \\
\text { Additional }\end{array}$ & $\begin{array}{c}\text { Dry } \\
\text { Nattiral } \\
\text { Gas } \\
\text { Proved } \\
\text { Pear Reserves }\end{array}$ & $\begin{array}{c}\text { Natural } \\
\text { Gass } \\
\text { Liquids } \\
\text { Proved } \\
\text { Reserves }\end{array}$ \\
\hline
\end{tabular}

\begin{tabular}{|c|c|c|c|c|}
\hline \multicolumn{5}{|c|}{ New Mexlco - West } \\
\hline 1977 & 29 & 2 & 8,152 & NA \\
\hline 1978 & 25 & 2 & 8.799 & NA \\
\hline 1979 & 21 & 0 & 9,690 & 321 \\
\hline $19 B 0$ & 29 & 0 & 9.757 & 332 \\
\hline 1981 & 33 & 0 & 10,272 & 346 \\
\hline 1982 & 26 & 0 & 8,966 & 322 \\
\hline 1983 & 34 & 0 & 8,446 & 319 \\
\hline 1984 & 35 & 0 & 8,167 & 290 \\
\hline 1965 & 45 & 1 & 7,866 & 236 \\
\hline 1986 & 51 & 0 & 8,114 & 360 \\
\hline 1987 & 46 & 5 & 8,739 & 579 \\
\hline 1988 & 40 & 6 & 14,221 & 815 \\
\hline 1989 & 46 & 4 & 12,359 & 737 \\
\hline 1990 & 54 & 3 & 14,004 & 768 \\
\hline 1991 & 27 & 0 & 15,333 & 703 \\
\hline 1992 & 26 & 0 & 15,868 & 843 \\
\hline 1993 & 19 & 0 & 15,585 & 763 \\
\hline $19 \$ 4$ & 16 & 0 & 14,207 & 777 \\
\hline 1995 & 19 & 0 & 14,624 & 696 \\
\hline 1996 & 13 & 0 & 13,695 & 760 \\
\hline 1997 & 16 & 0 & 12,872 & 596 \\
\hline
\end{tabular}

\begin{tabular}{|c|c|c|c|c|}
\hline Yoar & $\begin{array}{c}\text { Crude Oll } \\
\text { Proved } \\
\text { Reserves }\end{array}$ & $\begin{array}{l}\text { Crude Oil } \\
\text { Indlcated } \\
\text { Addlitional } \\
\text { Peserves }\end{array}$ & $\begin{array}{c}\text { Dry } \\
\text { Naturof } \\
\text { Ges } \\
\text { Proved } \\
\text { Reverves }\end{array}$ & $\begin{array}{l}\text { Natural } \\
\text { Gas } \\
\text { Liquids } \\
\text { Proved } \\
\text { Reserves }\end{array}$ \\
\hline
\end{tabular}

\begin{tabular}{lrrrr}
\hline \multicolumn{5}{c}{ North Dakota } \\
\hline 1977 & 155 & 10 & 361 & NA \\
1978 & 162 & 4 & 374 & NA \\
1979 & 217 & 6 & 439 & 47 \\
1980 & 214 & 6 & 537 & 61 \\
1981 & 273 & 8 & 581 & 66 \\
1982 & 237 & 8 & 629 & 71 \\
1983 & 258 & 53 & 600 & 69 \\
1984 & 260 & 54 & 566 & 73 \\
1985 & 255 & 34 & 569 & 74 \\
1986 & 218 & 35 & 541 & 69 \\
1987 & 215 & 33 & 508 & 67 \\
1988 & 216 & 39 & 541 & 52 \\
1989 & 246 & 31 & 561 & 59 \\
1990 & 285 & 0 & 586 & 60 \\
1991 & 232 & 4 & 472 & 56 \\
1992 & 237 & 3 & 496 & 64 \\
1993 & 226 & 7 & 525 & 55 \\
1994 & 226 & 2 & 507 & 55 \\
1995 & 233 & 6 & 463 & 53 \\
1996 & 248 & 6 & 462 & 48 \\
1997 & 279 & 6 & 479 & 47
\end{tabular}

\begin{tabular}{|c|c|c|c|c|c|c|c|c|c|}
\hline \multicolumn{5}{|c|}{ New York } & \multicolumn{5}{|c|}{ Ohio } \\
\hline 1977 & NA & $\mathbf{N A}$ & 165 & $\mathrm{NA}$ & 5977 & 74 & 0 & 495 & NA \\
\hline 1978 & NA & NA & 190 & NA & 1978 & 69 & 0 & 684 & NA \\
\hline 1979 & NA & MA & 211 & 0 & 1979 & *82 & 0 & $* 1,479$ & 0 \\
\hline 1980 & NA & NA & 208 & 0 & 1980 & $" 116$ & 0 & $-1,699$ & 0 \\
\hline 1981 & $\mathrm{NA}$ & $\mathrm{NA}$ & 264 & 0 & 1981 & $\star 112$ & 0 & 965 & 0 \\
\hline 1982 & $\mathrm{NA}$ & $\mathrm{NA}$ & 229 & NA & 1982 & 111 & 0 & $1,14 t$ & NA \\
\hline 1983 & NA & NA & 295 & $\mathrm{NA}$ & 1983 & $1 \$ 0$ & 0 & 2,030 & NA \\
\hline $19 \$ 4$ & NA & NA & 389 & NA & 1984 & -116 & 0 & 1,547 & NA \\
\hline 1965 & NA & NA & -369 & $N A$ & 1985 & 79 & 0 & 1,331 & MA \\
\hline 1986 & $\mathrm{NA}$ & $\mathrm{NA}$ & 457 & NA & 1986 & 72 & 0 & 1,420 & $\mathrm{NA}$ \\
\hline 1987 & NA & NA & 410 & NA & 1987 & 66 & 0 & 1,069 & NA \\
\hline 1988 & NA & NA & 351 & NA & $198 \mathrm{~B}$ & 64 & 0 & 1,229 & NA \\
\hline $19 \mathrm{Bg}$ & NA & NA & 368 & $N A$ & 1989 & 56 & 0 & 1,275 & NA \\
\hline 1990 & NA & NA & 354 & $\mathrm{NA}$ & 1990 & 65 & 0 & 1,214 & NA \\
\hline 1991 & NA & NA & 331 & NA & 1991 & 66 & 0 & 1,181 & NA \\
\hline 1992 & $\mathrm{NA}$ & NA & 329 & NA & 1992 & 68 & 0 & 1,161 & NA \\
\hline 1993 & NA & NA & "264 & $\mathrm{NA}$ & 1993 & 54 & 0 & 1,104 & NA \\
\hline 1994 & NA & NA & 242 & NA & 1994 & 58 & 0 & 1,094 & NA \\
\hline 1995 & NA & NA & 197 & NA & 1905 & E3 & 0 & 1,054 & NA \\
\hline 1955 & NA & NA & 232 & NA & 1996 & 53 & 0 & $1,1+3$ & NA \\
\hline 1997 & NA & NA & ${ }^{\star} 224$ & NA & 1997 & +43 & 0 & 985 & NA \\
\hline
\end{tabular}




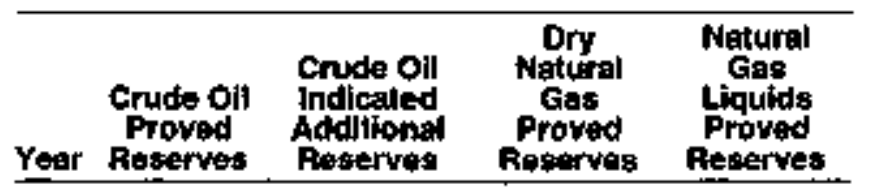

\begin{tabular}{|c|c|c|c|c|}
\hline Yoar & $\begin{array}{l}\text { Crude oil } \\
\text { Proved } \\
\text { Reserves }\end{array}$ & $\begin{array}{l}\text { Crude all } \\
\text { lndicated } \\
\text { Addlitine: } \\
\text { Reserves }\end{array}$ & $\begin{array}{c}\text { Dry } \\
\text { Nalural } \\
\text { Gas } \\
\text { Proved } \\
\text { Peserves }\end{array}$ & $\begin{array}{l}\text { Natural } \\
\text { Gas } \\
\text { Liquids } \\
\text { Proved } \\
\text { Poserves }\end{array}$ \\
\hline
\end{tabular}

\begin{tabular}{rrrrr}
\hline & \multicolumn{4}{c}{ Oklahoma } \\
\hline 1977 & 1,109 & 69 & 13,889 & NA \\
1978 & 979 & 33 & 14,417 & NA \\
1979 & 1,014 & 35 & 13,816 & 583 \\
1980 & 930 & 27 & 13,136 & 604 \\
1981 & 950 & 43 & 14,699 & 631 \\
1982 & 971 & 25 & 16,207 & 745 \\
1983 & 931 & 27 & 16,211 & 829 \\
1984 & 940 & 40 & 16,126 & 769 \\
1985 & 935 & 97 & 16,040 & 626 \\
1986 & 674 & 35 & 16,685 & 657 \\
1997 & 788 & 56 & 16,711 & 781 \\
1989 & 796 & 79 & 16,495 & 765 \\
1989 & 769 & 63 & 15,916 & 654 \\
1990 & 734 & 37 & 16,151 & 657 \\
1991 & 700 & 54 & 14,725 & 628 \\
1992 & 698 & 54 & 13,926 & 629 \\
1993 & 690 & 40 & 13,289 & 643 \\
1994 & 689 & 47 & 13,497 & 652 \\
1995 & 676 & 48 & 13,438 & 674 \\
1996 & 632 & 43 & 13,074 & 684 \\
1997 & 605 & 20 & 13,439 & 685
\end{tabular}

\begin{tabular}{|c|c|c|c|c|}
\hline & & Text: & tral & \\
\hline $\begin{array}{l}1977 \\
1978\end{array}$ & $\begin{array}{l}9.751 \\
8.911\end{array}$ & $\begin{array}{l}637 \\
533\end{array}$ & $\begin{array}{l}56,422 \\
55,583\end{array}$ & $\begin{array}{l}\text { NA } \\
\text { NA }\end{array}$ \\
\hline$\uparrow 979$ & 8,284 & 471 & 53,021 & 2,482 \\
\hline 1980 & 6,206 & 364 & 50,287 & 2,452 \\
\hline 1981 & $B, 093$ & 459 & 50,469 & 2,646 \\
\hline 1982 & 7,616 & 377 & 49,757 & 2,771 \\
\hline 1983 & 7,539 & 421 & 50,052 & 3,038 \\
\hline 1984 & 7,557 & 735 & 49,883 & 3,048 \\
\hline 1985 & 97,782 & 609 & 941,775 & G2,961 \\
\hline 1996 & 97,152 & 1,270 & 940,574 & 92,964 \\
\hline 1967 & $97,+12$ & $1,02 B$ & $9_{38,711}$ & $92, \mathrm{B2} 2$ \\
\hline 1988 & 97,043 & 1,099 & $9_{\beta B, 16 ?}$ & 92,617 \\
\hline 1989 & 96,966 & 806 & 938,381 & 92,563 \\
\hline 1990 & 97,106 & 618 & 938,192 & $9_{2,575}$ \\
\hline 1991 & 96,797 & 756 & 936,174 & 92,493 \\
\hline 1992 & 96,441 & 9612 & 935,093 & 92,402 \\
\hline 1993 & $9_{6,171}$ & 9581 & 934,718 & $9_{2,469}$ \\
\hline 1994 & 96,847 & 9491 & 935,974 & 92,414 \\
\hline 1995 & 95,743 & 9395 & 936,542 & $\$ 2,524$ \\
\hline 1996 & 95,736 & 9358 & $9_{38,270}$ & 92,606 \\
\hline 1997 & 96,687 & 9479 & $\mathbf{g}_{37,761}$ & 92,687 \\
\hline
\end{tabular}

9Exuludes Foderal oftishore; now Included in Foderal Ottshore. Gulf of Moxico (Texess).

\begin{tabular}{|c|c|c|c|c|c|c|c|c|c|}
\hline \multicolumn{5}{|c|}{ Peninaylyanin } & \multicolumn{5}{|c|}{ Texts - RRC District I } \\
\hline 1977 & $\$ 57$ & 0 & 769 & NA & 1977 & $r 174$ & 0 & 1,319 & NA \\
\hline 1978 & 27 & 0 & 899 & NA & 1978 & 111 & 2 & 986 & NA \\
\hline 1979 & 33 & 0 & ${ }^{n} 1,515$ & 1 & 1979 & 110 & 0 & 919 & 23 \\
\hline $19 \mathrm{gOO}$ & 35 & 0 & 951 & 0 & 1960 & $\cdot 150$ & 0 & 829 & 24 \\
\hline$\$ 981$ & 32 & 0 & $=1,264$ & 0 & 1981 & 127 & 5 & $\because 1,022$ & 26 \\
\hline 1982 & 37 & 0 & 1,429 & NA & 1982 & 129 & 6 & 892 & 29 \\
\hline 1983 & 41 & 0 & 1,882 & NA & 1983 & 165 & 6 & 1,087 & 43 \\
\hline 1994 & $=40$ & 0 & 1,575 & $\mathrm{NA}$ & 1984 & 173 & 4 & 838 & 39 \\
\hline 1995 & -38 & 0 & $-1,617$ & NA & 1985 & 177 & $\mathbf{B}$ & 967 & 40 \\
\hline 1986 & +26 & 0 & 1,560 & 1 & 1986 & 144 & 1 & 913 & 35 \\
\hline 1997 & 26 & 0 & $t, 647$ & NA & $10 \mathrm{~g} 7$ & 143 & 1 & 812 & 27 \\
\hline 1988 & -27 & 0 & 2,072 & NA & 1988 & 136 & 1 & 1,173 & 30 \\
\hline 1989 & 26 & 0 & 1,642 & NA & 1989 & 139 & 1 & 1,267 & 25 \\
\hline 1990 & 20 & 0 & 1,720 & NA & 1090 & 252 & 0 & 1,048 & 26 \\
\hline 1991 & 15 & 0 & 1,629 & NA & 1991 & 227 & 0 & 1,030 & $2 B$ \\
\hline 1992 & 16 & 0 & 1,528 & NA & 1992 & 185 & 0 & 933 & 27 \\
\hline 1993 & 14 & 0 & 1,717 & NA & 1993 & 133 & 0 & 698 & 26 \\
\hline 1994 & 15 & 0 & $1_{T} 800$ & NA & 1994 & 100 & 1 & 703 & 26 \\
\hline 1995 & 11 & 0 & 1,482 & NA & 1995 & 90 & 6 & 712 & 26 \\
\hline 1996 & 10 & 0 & 1,696 & NA & 1996 & $B 6$ & 1 & 906 & 46 \\
\hline 1997 & 17 & 0 & 1,852 & NA & 1997 & $B 3$ & $<1$ & 953 & 54 \\
\hline
\end{tabular}




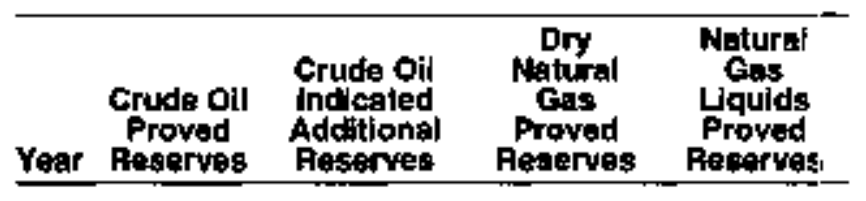

\begin{tabular}{|c|c|c|c|c|}
\hline Year & $\begin{array}{l}\text { Crude Oall } \\
\text { Proved } \\
\text { Peserves }\end{array}$ & $\begin{array}{l}\text { Crudit Oil } \\
\text { Indlcoted } \\
\text { Additional } \\
\text { Reserwes }\end{array}$ & $\begin{array}{c}\text { Dry } \\
\text { Natural } \\
\text { Gas } \\
\text { Proved } \\
\text { Reserves }\end{array}$ & $\begin{array}{l}\text { Natural } \\
\text { Gas } \\
\text { Lquids } \\
\text { Proved } \\
\text { Reserves }\end{array}$ \\
\hline
\end{tabular}

\begin{tabular}{|c|c|c|c|c|c|c|c|c|c|}
\hline \multicolumn{5}{|c|}{ Texes - RAC District 2 Onshore } & \multicolumn{5}{|c|}{ Texas - RAC Dlstrict 4 Onshore } \\
\hline 1977 & 395 & 80 & 3,162 & NA & 1977 & 145 & 7 & 9,621 & NA \\
\hline 1978 & 334 & 1 & 2.976 & NA & 1978 & 123 & 3 & 9,031 & NA \\
\hline 1979 & 292 & 1 & 2,974 & 64 & 1979 & 113 & 4 & 8,326 & 248 \\
\hline $19 \$ 0$ & 252 & 1 & 2,502 & 64 & 1900 & 96 & 3 & 8,130 & 252 \\
\hline 1981 & 229 & 1 & 2,629 & 68 & 1981 & 97 & $\theta$ & 8,004 & 260 \\
\hline 1982 & 206 & 0 & 2,493 & 75 & 1982 & 87 & 7 & 8,410 & 289 \\
\hline 1983 & 190 & 0 & 2,534 & 99 & 1983 & 96 & 3 & 8,316 & 292 \\
\hline 1964 & 192 & $<1$ & $2,5+2$ & 103 & 1984 & 99 & 3 & 8,525 & 295 \\
\hline 1985 & $16 \mathrm{~B}$ & 0 & 2,358 & 100 & 1985 & 98 & 2 & 8,250 & 269 \\
\hline 1986 & 148 & $<1$ & 2,180 & 89 & 1986 & 87 & 2 & 8,274 & 281 \\
\hline 1987 & 137 & 0 & 2,273 & 102 & 1987 & 80 & 2 & 7,490 & 277 \\
\hline t9a8 & 117 & 0 & 2,097 & 92 & 1988 & 65 & 1 & 7,029 & 260 \\
\hline 1989 & 107 & 0 & 1,770 & 72 & 1989 & 77 & $<1$ & 7,111 & 280 \\
\hline 1990 & 91 & 0 & 1,737 & 80 & 1990 & 67 & $<1$ & 7,475 & 279 \\
\hline 1991 & 90 & 0 & 1,390 & 75 & 1991 & 52 & $<1$ & 7,048 & 273 \\
\hline 1992 & 86 & 0 & 1,389 & 80 & 1992 & 50 & $<1$ & 6,739 & 272 \\
\hline 1993 & $\pi$ & 0 & 1,321 & 86 & 1993 & 69 & $<1$ & 7,036 & 278 \\
\hline 1994 & 74 & 0 & 1,360 & 86 & 1994 & 41 & $<1$ & 7.547 & 290 \\
\hline 1995 & 61 & 0 & 1,251 & 93 & 1995 & 50 & $<1$ & 7.709 & 287 \\
\hline t996 & 63 & $<1$ & 1,322 & 93 & 1996 & 51 & 0 & 7,769 & 323 \\
\hline 1997 & 66 & 0 & 1,634 & 87 & 1997 & 70 & $<1$ & 8,099 & 347 \\
\hline
\end{tabular}

\begin{tabular}{|c|c|c|c|c|c|c|c|c|c|}
\hline \multicolumn{5}{|c|}{ Texas - ARC Distriet 3 Onshore } & \multicolumn{5}{|c|}{ Texas - ARC Dlstrlet 5} \\
\hline 1977 & 937 & 33 & 7,518 & NA & 1977 & 88 & 0 & 931 & NA \\
\hline 1978 & 794 & 22 & 7,166 & NA & 1978 & 78 & 0 & $-1,298$ & NA \\
\hline 1979 & 630 & 32 & 6,915 & 231 & 1979 & 55 & 1 & 1,155 & 34 \\
\hline 1990 & 581 & 11 & 5.531 & 216 & 1980 & 52 & 0 & 1,147 & 44 \\
\hline 1981 & 552 & 11 & 5,292 & 230 & 1981 & 49 & 0 & 1,250 & 48 \\
\hline 1982 & 509 & 22 & 4,756 & 265 & 1982 & 45 & 0 & 1,308 & 5 \\
\hline 1983 & 517 & 27 & 4,680 & 285 & 7983 & 42 & 0 & 1,448 & 73 \\
\hline 1984 & 522 & 25 & 4,700 & 270 & 1984 & 36 & 51 & 1,874 & 74 \\
\hline 1985 & 471 & 6 & 4,180 & 260 & 1985 & +59 & 1 & 2,058 & 77 \\
\hline $19 \% 6$ & 420 & 3 & 3,753 & 237 & 1986 & 53 & 1 & 2,141 & $B$ \\
\hline 1987 & 396 & 4 & 3,632 & 241 & 1987 & 54 & 0 & 2,119 & 86 \\
\hline 1988 & 360 & 16 & 3,422 & $20 \mathrm{~B}$ & 1988 & 48 & 0 & 1,996 & 81 \\
\hline 1989 & 307 & 11 & 3,233 & 213 & 1989 & 46 & 0 & 1,845 & 80 \\
\hline 1990 & 275 & 13 & 2,894 & 187 & 1990 & 47 & 0 & 1,675 & B1 \\
\hline 1991 & 300 & 28 & 2,885 & 208 & 1991 & 46 & 0 & 1,863 & 71 \\
\hline 1992 & 304 & 27 & 2,684 & 211 & 1992 & 55 & 0 & 1,747 & 71 \\
\hline 1993 & 327 & 31 & 2,972 & 253 & $1 \operatorname{tgg} \beta$ & 52 & 0 & $t, 867$ & 64 \\
\hline 1994 & 330 & 61 & 3,366 & 254 & 1994 & 49 & 0 & 2,011 & 59 \\
\hline 1995 & 267 & 27 & 3,866 & 272 & 1995 & 34 & 0 & 1,682 & 54 \\
\hline 1996 & 281 & 27 & 4,349 & 289 & 1996 & 29 & 0 & 2,079 & 54 \\
\hline 1997 & 259 & 28 & 4.172 & 286 & 1997 & 54 & 0 & $\dagger, 710$ & 35 \\
\hline
\end{tabular}




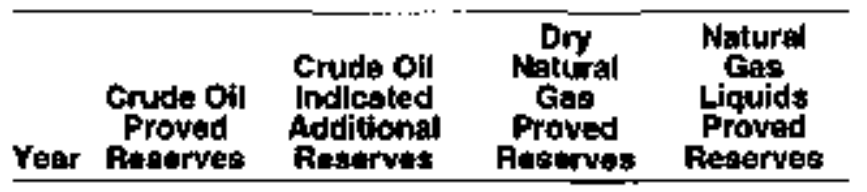

\begin{tabular}{|c|c|c|c|c|}
\hline Year & $\begin{array}{l}\text { Crude Oil } \\
\text { Proved } \\
\text { Peeidrves }\end{array}$ & $\begin{array}{l}\text { Crude oil } \\
\text { Indicated } \\
\text { Acditlonal } \\
\text { Reserves }\end{array}$ & $\begin{array}{c}\text { Dry } \\
\text { Natural } \\
\text { Guss } \\
\text { Proved } \\
\text { Roeervee }\end{array}$ & $\begin{array}{l}\text { Natural } \\
\text { Gas } \\
\text { Liquids } \\
\text { Proved } \\
\text { Recerves }\end{array}$ \\
\hline
\end{tabular}

\begin{tabular}{lrrrr}
\hline \multicolumn{5}{c}{ Texas - RRC District 6 } \\
\hline 1977 & 1,568 & 12 & 3,214 & NA \\
1978 & 1,444 & 3 & 3,240 & NA \\
1979 & 1,177 & 6 & 3,258 & 272 \\
1990 & 1,115 & 6 & 4,230 & 321 \\
1981 & 1,040 & 7 & 4,177 & 308 \\
1982 & 947 & 6 & 4,326 & 278 \\
1993 & 918 & 5 & 4,857 & 342 \\
1984 & 889 & 5 & 4,703 & 298 \\
1985 & 851 & 4 & 4,822 & 293 \\
1986 & 750 & 2 & 4,854 & 277 \\
1987 & 733 & 3 & 4,682 & 264 \\
1968 & 685 & 5 & 4,961 & 263 \\
1989 & 631 & 4 & 5,614 & 266 \\
1990 & 605 & 6 & 5,763 & 247 \\
1991 & 504 & 7 & 5,233 & 243 \\
1992 & 442 & 7 & 5,317 & 251 \\
1993 & 406 & $<1$ & 5,508 & 248 \\
1994 & 424 & $<1$ & 5,381 & 265 \\
1995 & 409 & 1 & 5,726 & 271 \\
1996 & $\$ 59$ & 1 & 5,899 & 290 \\
1997 & 348 & 1 & 5,867 & 260
\end{tabular}

\begin{tabular}{lllll}
\hline \multicolumn{5}{c}{ Texas - RRC Digtrict 7C } \\
\hline 1977 & 191 & NA & 2,831 & NA \\
1978 & 202 & NA & 2,821 & NA \\
1979 & 206 & NA & 2,842 & 182 \\
1980 & 207 & NA & 2,378 & 135 \\
1991 & 230 & NA & 2,503 & 196 \\
1982 & 229 & NA & 2,659 & 199 \\
1983 & 229 & NA & 2,568 & 219 \\
1984 & 240 & 24 & 2,866 & 233 \\
1985 & 243 & 21 & 2,914 & 256 \\
1996 & 213 & 22 & 2,721 & 246 \\
1987 & 220 & 26 & 2,708 & 243 \\
1988 & 212 & 31 & 2,791 & 238 \\
1989 & 247 & 16 & 3,180 & 238 \\
1990 & 274 & 8 & 3,514 & 256 \\
1991 & 253 & 9 & 3,291 & 241 \\
1992 & 255 & 33 & 3,239 & 269 \\
1993 & 199 & 15 & 3,215 & 273 \\
1994 & 221 & 14 & 3,316 & 265 \\
1995 & 204 & 8 & 3,107 & 274 \\
1996 & 219 & 5 & 3,655 & 303 \\
1997 & 227 & 4 & 3,407 & 327
\end{tabular}

\begin{tabular}{|c|c|c|c|c|c|c|c|c|c|}
\hline \multicolumn{5}{|c|}{ Texas - RAC Dlsitrlet 7B } & \multicolumn{5}{|c|}{ Texas - RAC Dlstrlet $\mathrm{B}$} \\
\hline 1977 & 250 & NA & 699 & NA & 1977 & 2,915 & 127 & 17,728 & NA \\
\hline 1978 & 190 & NA & 743 & NA & 1978 & 2,795 & 102 & 11,093 & NA \\
\hline 1979 & 208 & NA & 751 & 64 & 1979 & 2,686 & 88 & 10,077 & 505 \\
\hline 1980 & 196 & NA & 745 & 85 & 1980 & 2,597 & 86 & 9,144 & 498 \\
\hline 1991 & 254 & NA & 804 & 102 & 1981 & 2,503 & 105 & 8,546 & 537 \\
\hline 1982 & 199 & NA & 805 & 105 & 1982 & 2,312 & 75 & 8,196 & 588 \\
\hline 1986 & 217 & $\mathrm{NA}$ & 1,027 & 133 & 1983 & 2,350 & 99 & 8,156 & 681 \\
\hline 1984 & 218 & 62 & 794 & 106 & 1904 & 2,342 & 363 & 7,343 & 691 \\
\hline 1995 & 239 & 63 & 708 & 104 & 1985 & 2,333 & 325 & 7,330 & 665 \\
\hline 1996 & 193 & 64 & 684 & 109 & 1926 & 2,183 & 592 & 7,333 & 717 \\
\hline 1997 & 200 & 46 & 697 & 92 & 1987 & 2,108 & 399 & 6,999 & 640 \\
\hline 1988 & 205 & 42 & 704 & 98 & 1988 & 2,107 & 412 & 7,058 & 547 \\
\hline 1989 & 204 & 11 & 459 & 73 & 1989 & 2,151 & 366 & 6,753 & 554 \\
\hline 1990 & 198 & 8 & 522 & 76 & 1990 & 2,152 & 282 & 6,614 & 558 \\
\hline 1991 & 184 & 8 & 423 & 82 & 1991 & 2,114 & 328 & 6,133 & 477 \\
\hline 1992 & 163 & 11 & 455 & 68 & 1992 & 2,013 & 260 & 5,924 & 444 \\
\hline 1993 & 171 & 7 & 477 & 79 & 1993 & 2,057 & 262 & 5,516 & 439 \\
\hline 1994 & 145 & 5 & 425 & 62 & 1994 & 2,002 & 256 & 5,442 & 414 \\
\hline 1995 & 126 & 4 & 440 & 70 & 1995 & 2,032 & 187 & 5,441 & 444 \\
\hline 1996 & 136 & 4 & 520 & 65 & 1996 & 2,079 & 217 & 5,452 & 429 \\
\hline 1997 & 155 & 3 & 478 & 59 & 1997 & 2,100 & 308 & 5,397 & 459 \\
\hline
\end{tabular}

Energy Information Administraklon 


\begin{tabular}{|c|c|c|c|c|}
\hline Year & $\begin{array}{c}\text { Crude oll } \\
\text { Proved } \\
\text { Peserver }\end{array}$ & $\begin{array}{l}\text { Crude ot| } \\
\text { Indicalod } \\
\text { Addilianal } \\
\text { Reoenves }\end{array}$ & $\begin{array}{l}\text { Dry } \\
\text { Naturat } \\
\text { Gas } \\
\text { Prowed } \\
\text { Reserves }\end{array}$ & $\begin{array}{l}\text { Natural } \\
\text { Gas } \\
\text { Liquids } \\
\text { Proved } \\
\text { Reserves }\end{array}$ \\
\hline
\end{tabular}

\begin{tabular}{cccc}
\hline Crude Oil & $\begin{array}{c}\text { Crude Oll } \\
\text { Indigatied }\end{array}$ & $\begin{array}{c}\text { Dry } \\
\text { Natural } \\
\text { Gas } \\
\text { Proved } \\
\text { Additional } \\
\text { Proved } \\
\text { Pear Reserves }\end{array}$ & $\begin{array}{c}\text { Natural } \\
\text { Gas } \\
\text { Liquids } \\
\text { Proved } \\
\text { Roserves }\end{array}$ \\
\hline
\end{tabular}

\begin{tabular}{|c|c|c|c|c|}
\hline \multicolumn{5}{|c|}{ Toxas - RAC Dlatrict BA } \\
\hline 1977 & 2,626 & 291 & 1,630 & NA \\
\hline 1978 & 2,439 & 330 & 1,473 & $N A$ \\
\hline 1979 & 2,371 & 270 & 1,055 & 351 \\
\hline 1900 & 2,504 & 196 & 1,057 & 290 \\
\hline 1991 & 2,538 & 247 & $1,07 t$ & 335 \\
\hline 1982 & 2,481 & 200 & 1,041 & 296 \\
\hline 1983 & 2,366 & 203 & 966 & 262 \\
\hline 1984 & 2,413 & 217 & 907 & 282 \\
\hline 1985 & 2,711 & 147 & 958 & 283 \\
\hline 1986 & 2,618 & 559 & 845 & 331 \\
\hline 1987 & 2,735 & 525 & 876 & 307 \\
\hline 1988 & 2,800 & 569 & 832 & 326 \\
\hline 1989 & 2,754 & 377 & 1,074 & $3 \$ 2$ \\
\hline 1990 & 2,847 & 285 & 1,096 & 354 \\
\hline 1991 & 2,763 & 363 & 1,073 & 333 \\
\hline 1992 & 2,500 & 273 & 1,239 & 267 \\
\hline 1998 & 2,435 & 264 & 1,043 & 298 \\
\hline 1994 & 2,223 & 154 & 1,219 & 267 \\
\hline 1995 & 2,233 & 156 & 041 & 284 \\
\hline 1996 & 2,207 & 99 & $93 t$ & 262 \\
\hline 1997 & 2,099 & 131 & 847 & 200 \\
\hline
\end{tabular}

\begin{tabular}{|c|c|c|c|c|}
\hline \multicolumn{5}{|c|}{ Texas - RRC District 10} \\
\hline 1977 & -120 & 4 & 7,744 & NA \\
\hline 1978 & 90 & 0 & 7,406 & NA \\
\hline 1979 & 97 & 2 & 6,784 & 375 \\
\hline 1980 & go & 2 & 6,435 & 369 \\
\hline 1961 & 107 & 2 & 6,229 & 364 \\
\hline 1982 & 112 & 2 & 6,210 & 391 \\
\hline 1983 & 105 & 6 & 5,919 & 413 \\
\hline 1984 & 100 & 6 & 5,467 & 440 \\
\hline 1985 & ${ }^{*} 140$ & 5 & 5,469 & 433 \\
\hline 1986 & $=104$ & 5 & 5,276 & 428 \\
\hline 1987 & 102 & 2 & 4,962 & 417 \\
\hline 1988 & 99 & 4 & 4,830 & 363 \\
\hline 1989 & 97 & 3 & 4,767 & 342 \\
\hline 1990 & 99 & 3 & 4,490 & $32 B$ \\
\hline 1991 & 95 & 2 & 4,509 & 356 \\
\hline 1992 & 89 & $<1$ & 4,409 & 336 \\
\hline 1990 & 83 & $<1$ & 4,040 & 329 \\
\hline 1994 & 75 & $<1$ & 4,246 & 326 \\
\hline 1995 & 80 & 6 & 4,436 & 353 \\
\hline 1996 & 74 & 4 & 4,391 & 332 \\
\hline 1997 & 79 & 4 & 4,004 & 382 \\
\hline
\end{tabular}

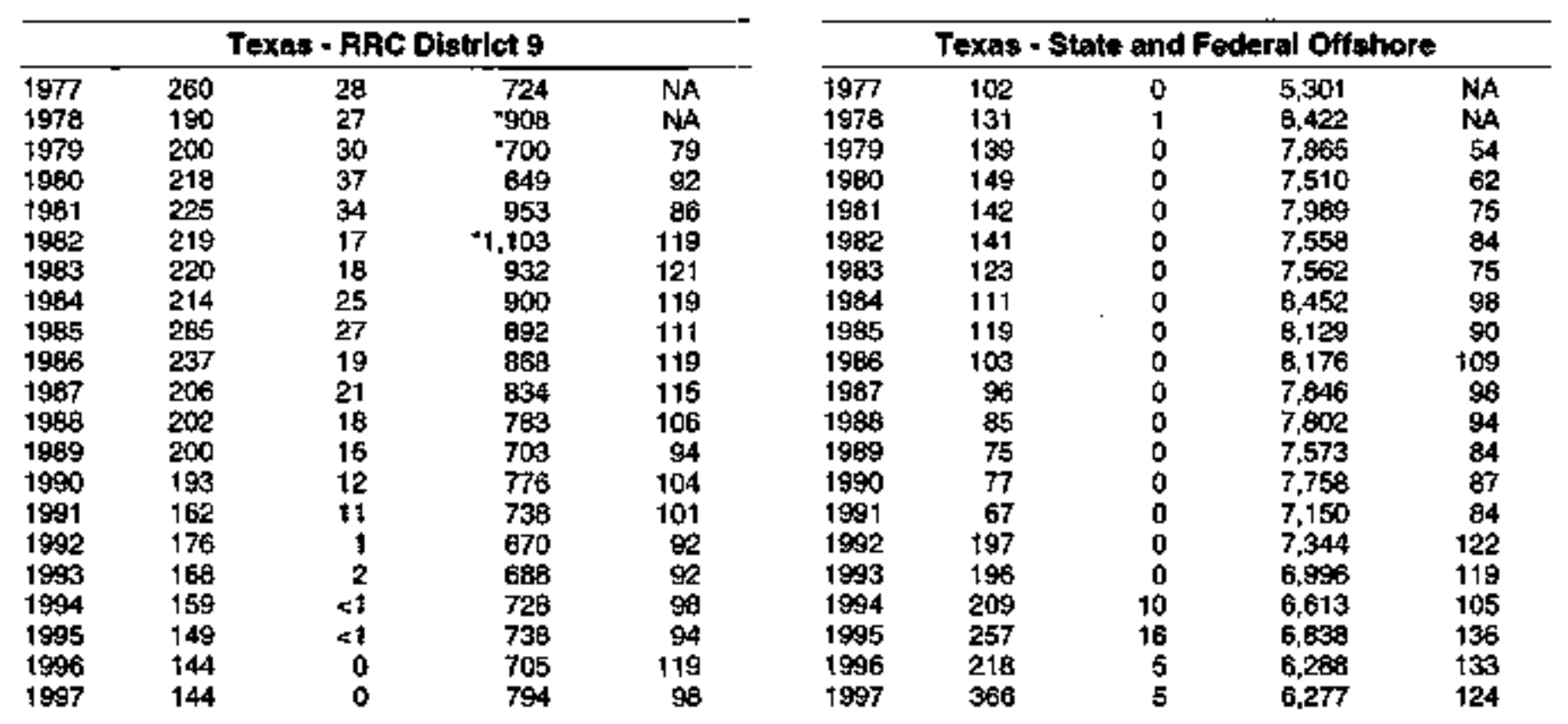




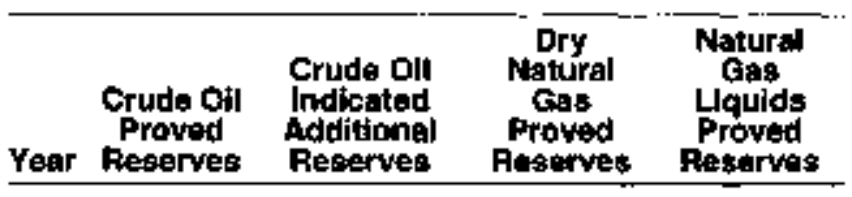

\begin{tabular}{cccc} 
Crude Oll & $\begin{array}{c}\text { Crude Oll } \\
\text { Proved } \\
\text { Addicated } \\
\text { Reserval }\end{array}$ & $\begin{array}{c}\text { Dry } \\
\text { Naturat } \\
\text { Gas } \\
\text { Proved } \\
\text { Reserves }\end{array}$ & $\begin{array}{c}\text { Natural } \\
\text { Gas } \\
\text { Liquids } \\
\text { Proved } \\
\text { Pleserves }\end{array}$ \\
\hline
\end{tabular}

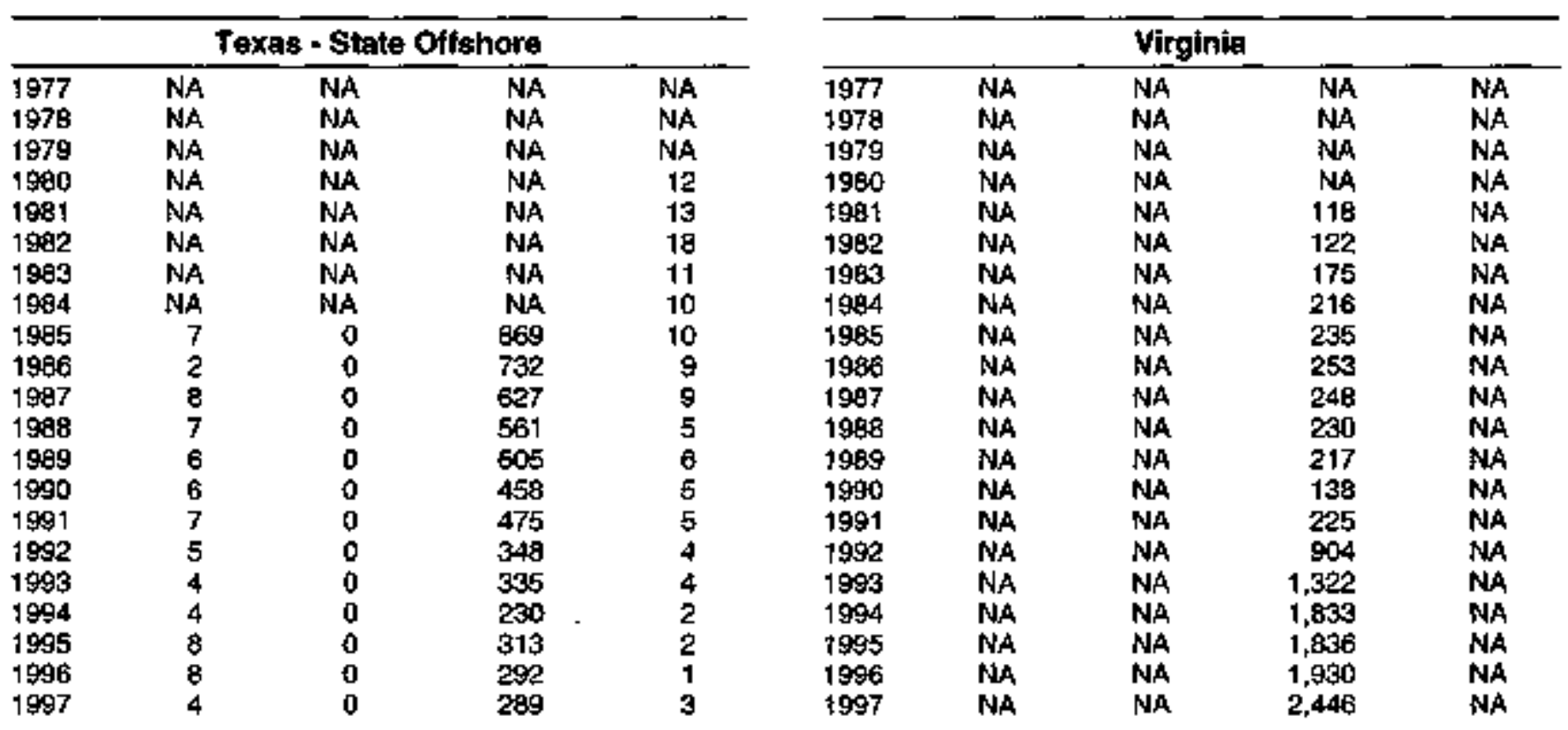

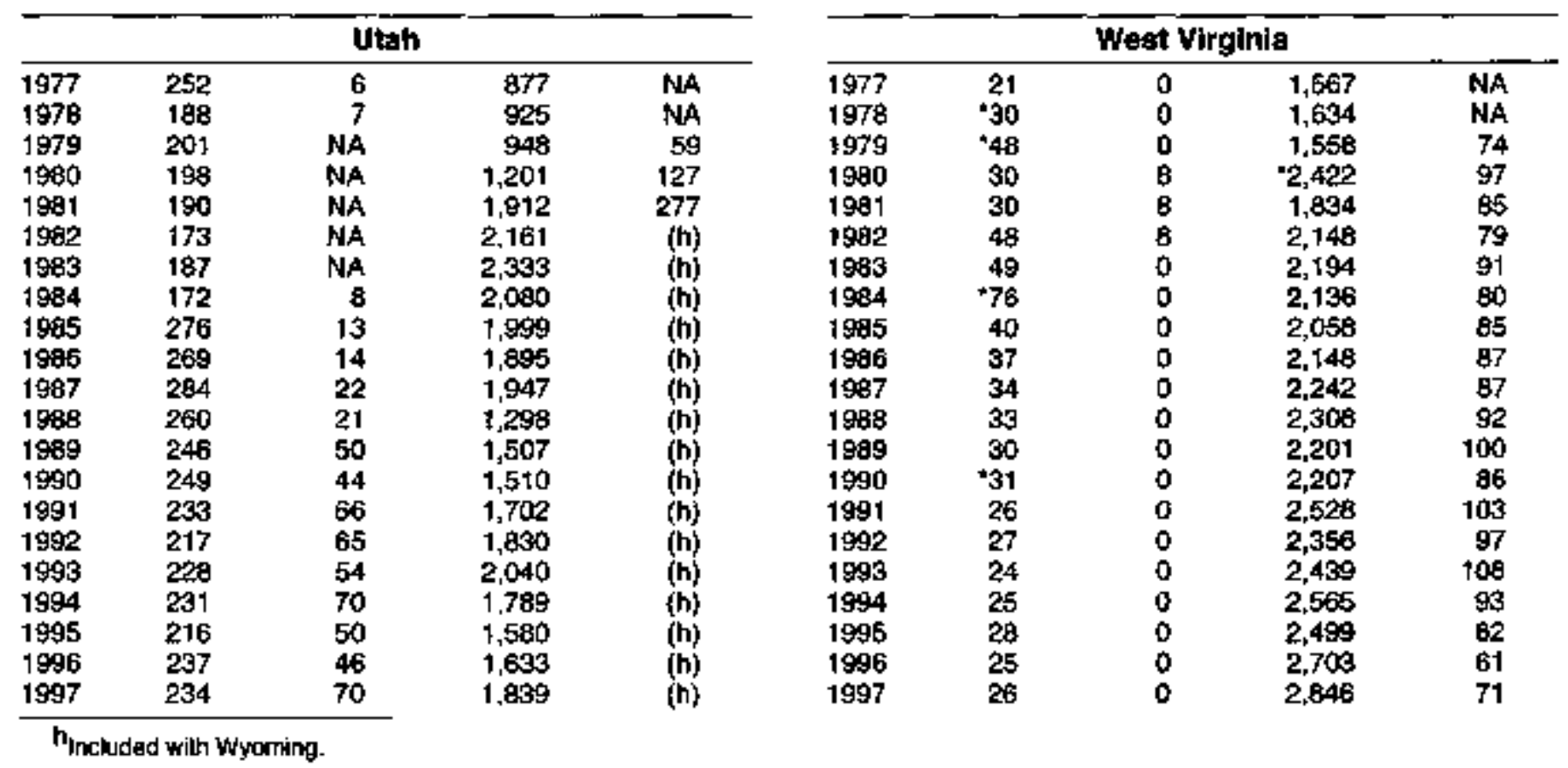




\begin{tabular}{|c|c|c|c|c|}
\hline Year & $\begin{array}{l}\text { Crude Oil } \\
\text { Proved } \\
\text { Rastrvess }\end{array}$ & $\begin{array}{l}\text { Conde okl } \\
\text { Indicaled } \\
\text { Additlonal } \\
\text { Ruserves }\end{array}$ & $\begin{array}{c}\text { Dry } \\
\text { Natural } \\
\text { Gas } \\
\text { Proved } \\
\text { Reserves }\end{array}$ & $\begin{array}{l}\text { Natural } \\
\text { Gaps } \\
\text { Liquids } \\
\text { Proved } \\
\text { Roserves }\end{array}$ \\
\hline
\end{tabular}

\begin{tabular}{|c|c|c|c|c|}
\hline Yerar & $\begin{array}{l}\text { Crude oll } \\
\text { Proved } \\
\text { Roserves }\end{array}$ & $\begin{array}{l}\text { Crude Oij } \\
\text { Anchieated } \\
\text { Additional } \\
\text { Reserves }\end{array}$ & $\begin{array}{l}\text { Dry } \\
\text { Niatural } \\
\text { Cass } \\
\text { Proved } \\
\text { Pisserves }\end{array}$ & $\begin{array}{c}\text { Netural } \\
\text { Cas } \\
\text { Lisusids } \\
\text { Proved } \\
\text { Reserves }\end{array}$ \\
\hline
\end{tabular}

\begin{tabular}{|c|c|c|c|c|}
\hline \multicolumn{5}{|c|}{ Wyoming } \\
\hline 1977 & 851 & 31 & 6,305 & NA \\
\hline 1978 & 845 & 36 & 7.211 & NA \\
\hline 1979 & 941 & 40 & 7,526 & 285 \\
\hline 1980 & 928 & 28 & 9,100 & 341 \\
\hline 1981 & 840 & 53 & 9,307 & 384 \\
\hline 1992 & 856 & 58 & 9,758 & 681 \\
\hline 1903 & 957 & 61 & 10,227 & 789 \\
\hline 1984 & 954 & 71 & 10,482 & $i_{860}$ \\
\hline 1985 & 951 & 18 & 10,617 & İ49 \\
\hline 1986 & 849 & 126 & 9,756 & 950 \\
\hline 1997 & 854 & 27 & 10,023 & 1924 \\
\hline 1988 & 815 & 35 & 10,308 & $i_{1,154}$ \\
\hline 1909 & 825 & 46 & 10,744 & 896 \\
\hline 1990 & 794 & 42 & 9,944 & 812 \\
\hline 1991 & 757 & 24 & 9,941 & 748 \\
\hline 1992 & $6 \mathrm{ES}$ & $1 B$ & 10,826 & $i_{000}$ \\
\hline 1993 & 624 & 12 & 10,933 & 1600 \\
\hline 1994 & 565 & 13 & 10,879 & 564 \\
\hline 1995 & 605 & 12 & 12,166 & 590 \\
\hline 1996 & 600 & 14 & 12,320 & 727 \\
\hline 1997 & 627 & 11 & 13,562 & 1761 \\
\hline
\end{tabular}

\begin{tabular}{lcccc}
\hline \multicolumn{5}{c}{ Federal Ofishore - Pacific (Calfiornis) } \\
\hline 1995 & 991 & NA & 1,119 & 12 \\
1986 & 974 & 2 & 1,325 & 15 \\
1987 & 1,037 & 2 & 1,452 & 17 \\
1998 & 1,024 & 0 & 1,552 & 21 \\
1989 & 987 & 0 & 1,496 & 25 \\
1990 & 962 & 0 & 1,454 & 16 \\
1991 & 785 & 0 & 1,162 & 16 \\
1992 & 734 & 0 & 1,118 & 20 \\
1993 & 673 & 0 & 1,099 & 25 \\
1994 & 653 & 0 & 1,170 & 21 \\
1995 & 571 & 0 & 1,265 & 25 \\
1996 & 518 & 0 & 1,244 & 23 \\
1997 & 526 & 0 & 544 & 14 \\
\hline
\end{tabular}

Note: Deata not tabulated for yeers $1977-1984$.

iulah and wyoming are comblnect.

\begin{tabular}{lllll}
\hline & \multicolumn{3}{c}{ Fedieral Offshore - Total } & \\
\hline 1986 & 2,862 & 11 & 34,492 & 702 \\
1986 & 2,715 & 16 & 34,223 & 681 \\
1987 & 2,639 & 21 & 31,931 & 638 \\
1988 & 2,629 & 21 & 32,264 & 622 \\
1989 & 2,747 & 32 & 32,651 & 678 \\
1990 & 2,805 & 49 & 31,433 & 619 \\
1991 & 2,620 & 18 & 29,448 & 640 \\
1992 & 2,569 & 31 & 27,767 & 610 \\
1993 & 2,745 & 18 & 27,143 & 630 \\
1994 & 2,760 & 53 & 28,388 & 624 \\
1995 & 3,089 & 62 & 29,182 & 655 \\
1996 & 3,095 & 45 & 29,096 & 776 \\
1997 & 3,477 & 41 & 29,466 & 920 \\
\hline
\end{tabular}

Jinciudes Slale otlehore Alabema.

Note: Data not tabulated for yegrs $1977-1984$.

\begin{tabular}{|c|c|c|c|c|}
\hline \multicolumn{5}{|c|}{ Federal Offshore - Gulf of Mexlco (Loulsiana) } \\
\hline $\begin{array}{l}19 B 6 \\
1986 \\
1987 \\
1988 \\
1989 \\
1990 \\
1991 \\
1992 \\
1993 \\
1994 \\
1995 \\
1996 \\
1997\end{array}$ & $\begin{array}{l}1,759 \\
1,640 \\
1,514 \\
1,527 \\
1,691 \\
1,772 \\
1,775 \\
1,643 \\
1,890 \\
1,922 \\
2,269 \\
2,357 \\
2,587\end{array}$ & $\begin{array}{l}11 \\
14 \\
19 \\
21 \\
32 \\
49 \\
18 \\
31 \\
18 \\
43 \\
48 \\
40 \\
36\end{array}$ & $\begin{array}{l}f_{26,113} \\
f_{25,454} \\
f_{23,260} \\
f_{23,471} \\
f_{24,187} \\
k_{22,679} \\
k_{21,611} \\
k_{19,653} \\
k_{19,333} \\
k_{20,835} \\
k_{21,392} \\
k_{21,656} \\
k_{21,934}\end{array}$ & $\begin{array}{l}610 \\
566 \\
532 \\
512 \\
575 \\
k_{519} \\
k_{546} \\
k_{472} \\
k_{490} \\
k_{500} \\
k_{496} \\
k_{621} \\
k_{765}\end{array}$ \\
\hline
\end{tabular}




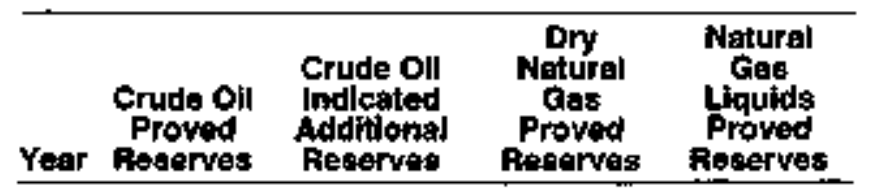

\begin{tabular}{|c|c|c|c|c|}
\hline Yeer & $\begin{array}{l}\text { Crude Oil } \\
\text { Proved } \\
\text { Reserves }\end{array}$ & $\begin{array}{l}\text { Crude otl } \\
\text { Indlcated } \\
\text { Additional } \\
\text { Rerenres }\end{array}$ & $\begin{array}{c}\text { Dry } \\
\text { Notural } \\
\text { Gas } \\
\text { Proved } \\
\text { Peserves }\end{array}$ & $\begin{array}{l}\text { Natural } \\
\text { coss } \\
\text { Llaculds } \\
\text { Proved } \\
\text { Reserves }\end{array}$ \\
\hline
\end{tabular}

\begin{tabular}{lcccc}
\hline \multicolumn{5}{c}{ Federai Offshore + Gulf of Mexico (Texas) } \\
\hline 1985 & 112 & 0 & 7,260 & 80 \\
1986 & 101 & 0 & 7,444 & 100 \\
1987 & 88 & 0 & 7,219 & 89 \\
1988 & 78 & 0 & 7,241 & 89 \\
1969 & 69 & 0 & 6,968 & 78 \\
1990 & 71 & 0 & 7,300 & 82 \\
1991 & 60 & 0 & 6,675 & 79 \\
1992 & 192 & 0 & 6,996 & 118 \\
1993 & 192 & 0 & 6,661 & 115 \\
1994 & 205 & 10 & 6,363 & 103 \\
1995 & 249 & 16 & 6,525 & 734 \\
1996 & 210 & 5 & 5,996 & 132 \\
1997 & 362 & 5 & 5,988 & 721 \\
\hline
\end{tabular}

\begin{tabular}{|c|c|c|c|c|}
\hline \multicolumn{5}{|c|}{ Miscellaneous } \\
\hline 1977 & 23 & 0 & 102 & NA \\
\hline 1978 & 24 & 0 & 109 & NA \\
\hline 1979 & 20 & 1 & $* 153$ & 2 \\
\hline 1980 & +38 & 0 & 176 & 3 \\
\hline 1991 & 40 & 7 & 191 & 21 \\
\hline 1982 & 33 & 0 & 69 & 4 \\
\hline 1983 & 30 & B & 78 & 5 \\
\hline 1984 & 23 & 0 & 75 & 5 \\
\hline 1995 & 35 & 0 & 76 & 3 \\
\hline 1986 & 33 & 0 & 133 & 2 \\
\hline 1887 & 30 & 0 & 65 & 4 \\
\hline 1988 & 34 & 0 & 83 & 5 \\
\hline 1999 & 39 & 0 & 83 & 5 \\
\hline 1990 & 43 & 1 & -70 & 3 \\
\hline 1991 & 42 & 5 & 75 & 8 \\
\hline 1992 & 29 & 0 & 92 & $\mathrm{~B}$ \\
\hline 1993 & 34 & 0 & 94 & 8 \\
\hline 1994 & 20 & 0 & 65 & B \\
\hline 1995 & ${ }^{*} 22$ & 0 & +69 & 7 \\
\hline 1996 & $\overline{18}$ & 0 & 67 & 7 \\
\hline 1997 & 19 & 0 & 43 & 9 \\
\hline
\end{tabular}

Nole: States knckuded may vary tor different report years ard inydrocarton typee.

\begin{tabular}{|c|c|c|c|c|}
\hline \multicolumn{5}{|c|}{ Lower 48 States } \\
\hline 1977 & 23,367 & 2,168 & 175,170 & NA \\
\hline 1978 & 21,971 & $\$, 964$ & 175,988 & NA \\
\hline 1979 & 20,935 & 1,878 & 163,738 & 6,592 \\
\hline 1980 & 21,054 & 1,622 & 165,639 & 6,717 \\
\hline 1981 & $21,14 \$$ & 1,694 & 169,693 & 7,058 \\
\hline 1932 & 20.452 & 1,478 & 166,522 & 7,212 \\
\hline 1983 & 20,428 & 1,548 & 165,964 & 7,693 \\
\hline 1984 & 20,883 & 1,956 & 162,987 & 7,624 \\
\hline 1985 & 21,360 & 1,682 & 159,522 & 7,561 \\
\hline 1986 & 20,014 & 2,597 & $15 \mathrm{~A}, 922$ & 7,784 \\
\hline 1987 & 19,078 & 3,064 & 153,986 & 7,729 \\
\hline 1988 & 19,886 & 3.169 & 168,946 & 7,837 \\
\hline 1989 & 19,827 & 2,999 & 158,177 & 7,389 \\
\hline 1990 & 19,730 & $2,5: 4$ & 160,046 & 7,246 \\
\hline 1991 & 18,599 & 2,810 & 157,509 & 7,104 \\
\hline 1992 & 17,723 & 2,451 & 155.377 & 7,104 \\
\hline 1993 & 17,182 & 2,292 & 152,508 & 6,901 \\
\hline 1994 & 16,690 & 2,129 & 154,104 & 6,869 \\
\hline 1995 & 18,771 & 2,087 & 155,649 & 7,090 \\
\hline 1996 & 16,743 & 1,924 & 157,180 & 7,486 \\
\hline 1997 & 17,385 & 2,375 & 156.661 & 7,342 \\
\hline
\end{tabular}

\begin{tabular}{llllr}
\hline & \multicolumn{4}{c}{ U,S. Total } \\
\hline 1977 & 31,780 & 3,014 & 207,413 & NA \\
1978 & 31,355 & 2,362 & 208,033 & NA \\
1979 & 29,810 & 2,276 & 200,997 & 5,615 \\
1980 & 29,805 & 1,622 & 199,021 & 6,728 \\
1981 & 29,426 & 1,594 & 201,730 & 7,068 \\
1982 & 27,659 & 1,478 & 201,512 & 7,221 \\
1983 & 27,735 & 2,124 & 200,247 & 7,901 \\
1984 & 28,446 & 2,326 & 197,463 & 7,643 \\
1985 & 28,416 & 2,041 & 193,369 & 7,944 \\
1986 & 26,869 & 3,499 & 191,586 & 8,165 \\
1987 & 27,256 & 3,649 & 187,211 & 8,147 \\
1988 & 26,875 & 3,600 & 168,024 & $B, 238$ \\
1989 & 26,501 & 3,749 & 167,116 & 7,769 \\
1990 & 26,264 & 3,483 & 169,346 & 7,586 \\
1991 & 24,682 & 4,266 & 167,062 & 7,464 \\
1992 & 23,745 & 3,782 & 165,015 & 7,451 \\
1993 & 22,957 & 3,453 & 162,415 & 7,228 \\
1994 & 22,457 & 3,151 & 163,837 & 7,170 \\
1995 & 22,351 & 2,669 & 165,146 & 7,399 \\
1996 & 22,017 & 2,876 & 166,474 & 7,623 \\
1997 & 22,546 & 3,207 & 167,223 & 7,973 \\
\hline
\end{tabular}


Table D1, U.S. Proved Reserves of Crude Oll, 1976-1997 (Milion Barrels of 42 U.S. Gallons)

\begin{tabular}{|c|c|c|c|c|c|c|c|c|c|c|c|}
\hline Yest & $\begin{array}{c}\text { At:uatumenta" } \\
\text { (1) }\end{array}$ & $\begin{array}{l}\text { Rervisian } \\
\text { incrititit: } \\
\text { (2) }\end{array}$ & 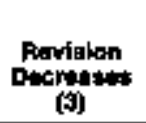 & 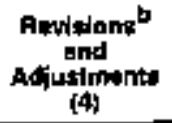 & $\begin{array}{c}\text { Extensians } \\
\text { (5) }\end{array}$ & $\begin{array}{l}\text { Now Fipld } \\
\text { Discoverlat } \\
\text { (6) }\end{array}$ & 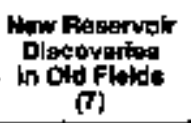 & $\begin{array}{c}\text { Totels } \\
\text { Dincowerien } \\
\text { (b) }\end{array}$ & $\begin{array}{c}\text { Production } \\
\text { for }\end{array}$ & 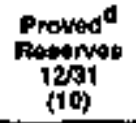 & $\begin{array}{l}\text { Chengen } \\
\text { Irom } \\
\text { Prior Year } \\
\text { (1) }\end{array}$ \\
\hline $197 \theta$ & - & - & - & - & - & - & - & - & - & ${ }^{3} 33,502$ & $=$ \\
\hline 197 & $f-40$ & 1,503 & 1,117 & 346 & 496. & 16 & 130 & 794 & 2,062 & 31,7的 & $-1,722$ \\
\hline 1978 & 366 & 2.799 & 1.409 & 1,756 & 444 & 267 & 116 & 827 & 3,028 & 31,355 & -425 \\
\hline 1979 & 337 & 2,438 & 2,001 & 774 & 424 & 108 & 104 & 536 & 2,956 & 20,810 & $-1,545$ \\
\hline 1980 & 210 & 2,683 & 994 & 2,108 & 572 & 143 & 147 & 692 & 2,975 & 29,805 & -5 \\
\hline 1981 & 138 & $2,15 t$ & 890 & 1,409 & 754 & 254 & 157 & 1.161 & 2.940 & 20,426 & -978 \\
\hline $19 \mathrm{A2}$ & -83 & 2,245 & $1, B 11$ & 351 & 634 & 204 & 193 & 1.031 & 2,950 & 27.858 & -1.588 \\
\hline 1983 & 462 & 2.810 & 1,299 & 1,979 & 6 6ᄅ & 105 & 190 & 924 & $\$, 020$ & 27,735 & -123 \\
\hline 1984 & 159 & 3,072 & 1,227 & 2,6004 & 744 & 242 & 158 & 1,144 & 3,0987 & 29.446 & +711 \\
\hline 1985 & 429 & 3.097 & 1.499 & $2,0,7$ & $74 \hat{2}$ & 84 & 169 & 395 & 3,052 & 20,416 & -30 \\
\hline 1968 & 57 & 2,724 & 1,889 & 912 & $4 C=$ & 48 & $\mathbf{B 1}$ & 634 & 2.973 & 26, & $-1,527$ \\
\hline 1987 & 293 & 3,687 & 1.371 & 2,549 & 484 & 96 & 111 & 691 & 2,073 & 27,256 & +367 \\
\hline 1989 & 364 & 3.684 & 1,221 & 1,827 & 355 ' & 71 & 127 & 553 & 2.811 & 26, & -431 \\
\hline 1989 & 213 & 2,698 & 1,385 & 1,540 & 514 & 112 & 50 & 716 & 2,580 & 20,501 & -324 \\
\hline 1900 & 86 & 2499 & 1,000 & 1,569 & 456 & 98 & 195 & 689 & 2,505 & 26.254 & -247 \\
\hline 1991 & 163 & 2,407 & 1,874 & 38 穴 & 305 & 97 & 92 & 554 & 2,512 & 24,682 & $-1,572$ \\
\hline 1962 & 290 & 1,804 & 1,060 & 1,025 & 391 & $\mathbf{B}$ & 85 & 484 & 2,446 & 23,745 & -937 \\
\hline 1993 & 271 & 2,011 & 1,518 & 706 & 356 & 319 & 110 & 785 & 2,339 & 22,957 & -780 \\
\hline 1994 & $18 \theta$ & 2,364 & 1,357 & 1,196 & 397 & 64 & 111 & 572 & 2,208 & 22,457 & -500 \\
\hline 1985 & 122 & 1,823 & 795 & 1.150 & $5 \infty 0$ & 114 & 343 & 857 & 2,219 & 29,951 & -106 \\
\hline 1998 & 175 & 1,723 & 986 & 912 & 543 & 243 & 941 & 927 & 2,173 & 22,017 & -334 \\
\hline 1997 & 520 & $\uparrow, 998$ & 1,084 & 1,434 & 477 & 697 & $11 \theta$ & 1.233 & 2,190 & $22,5 d 6$ & +529 \\
\hline
\end{tabular}

Includes operator reported corrections for the years 1976 though 1981. After 1981 operators included conrections with revislons.

"Fewsions and adjusimenls = Col. $1+$ Col. 2 - Col. 3.

Totel discoveries a Col. $5+$ Col, $6+$ Col. 7 .

Proved restrves = Cod. 10 from prior yeer + Col. 4 + Col. 8 - Col. 9 .

Based on following year data only.

'Consists bnly of operater reported corrections and no other achistrnents.

- - Nol applicable.

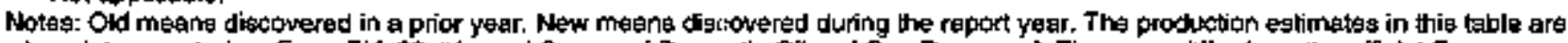
based on data reported on Form ElA-23. "Anmual Survey of Domerstic oll and Gas Reserves". They may ditter 1rom the officien Energy"

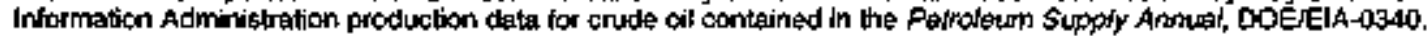

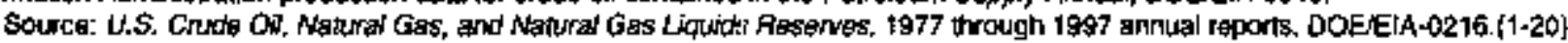


Table D2. U.S. Lower 49 Proved Reserves of Crude OA, 1976-1997 (Multion Barrels of 42 U.S. Gallons)

\begin{tabular}{|c|c|c|c|c|c|c|c|c|c|c|c|}
\hline Yalr & Actustimtio & 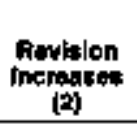 & $\begin{array}{c}\text { Revisan } \\
\text { Decrratiats } \\
\text { (a) }\end{array}$ & $\begin{array}{l}\text { Revigionts } \\
\text { and } \\
\text { Adjughments } \\
\text { (4) }\end{array}$ & $\begin{array}{c}\text { Exlontiont } \\
\text { (5) }\end{array}$ & 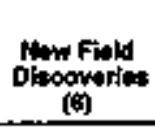 & 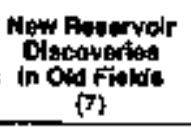 & $\begin{array}{c}\text { Totate } \\
\text { Diateorentod } \\
\text { (d) }\end{array}$ & $\begin{array}{c}\text { Productol } \\
\text { (\%) }\end{array}$ & $\begin{array}{l}\text { Proved } \\
\text { Reperyes } \\
+2 / 31 \\
\text { (10) }\end{array}$ & $\begin{array}{l}\text { Changes } \\
\text { Irom } \\
\text { Prior Yoar } \\
\text { (11) }\end{array}$ \\
\hline 1976 & - & - & - & - & - & - & - & $=$ & $=$ & $\theta_{24,920}$ & $=$ \\
\hline $19 \pi$ & $f_{-40}$ & 1,460 & 1,116 & 343 & 496 & 198 & 130 & 794 & 2,698 & 23,387 & $-1,561$ \\
\hline 1978 & -48 & 1,909 & 1,400 & 461 & $\$ 44$ & 142 & 116 & 702 & 2.559 & 21,971 & $-1,306$ \\
\hline 1979 & 342 & 2,404 & 1,975 & 771 & 424 & 108 & 104 & 636 & 2,443 & 20,935 & $-1,086$ \\
\hline 1980 & 210 & 2,505 & 981 & 1,734 & 479 & 143 & 147 & 769 & 2.504 & $21,05 d$ & $+\$ 19$ \\
\hline 1991 & 276 & 1,887 & 978 & 1,285 & 750 & 254 & $15 ?$ & 1,161 & 2,357 & 21,143 & +89 \\
\hline 1982 & -8 & 2,146 & 1,462 & 802 & 830 & 204 & t99 & 1,090 & 2,323 & 20,452 & -691 \\
\hline 1963 & 462 & 2,247 & 1,2006 & 1,411 & 625 & 105 & 190 & $\sec$ & $2, \$ \$ 5$ & 20,429 & -24 \\
\hline 1984 & 160 & 2,801 & 1,214 & 1,747 & 742 & 207 & 158 & 1,107 & 2,399 & 20,889 & +455 \\
\hline 19995 & 361 & 2,884 & 1,197 & 2,028 & 581 & Bd & 169 & क्ष\$4 & 2,366 & द 1,3000 & +477 \\
\hline 1986 & 70 & 2.001 & 1,642 & 429 & 390 & 48 & B1 & 528 & 2,303 & 20,014 & $-1,348$ \\
\hline 198 ? & 233 & 2,586 & 1,213 & 1,586 & 294 & 38 & 101 & 489 & 2,155 & 19.678 & -136 \\
\hline 1988 & 759 & 2,399 & 1.21 & 1,640 & 340 & 43 & $12 ?$ & 510 & $2,0 \mathrm{ert}$ & $19,8 \times 6$ & -12 \\
\hline 1989 & 216 & 2,438 & $1,3: 5$ & 1,327 & 342 & 106 & 97 & 537 & 1,903 & 19,627 & -99 \\
\hline 1990 & 151 & $1,909 ?$ & 996 & 1,152 & 371 & 98 & 135 & 604 & 1,863 & 19,730 & -97 \\
\hline 1981 & 164 & 1,898 & 1,848 & 214 & 37 & 97 & 87 & 511 & 1,858 & 18,599 & $-1,131$ \\
\hline 1992 & 297 & 1.343 & 1,096 & 574 & 279 & 日 & 84 & 371 & 1,821 & 17.723 & -976 \\
\hline 1989 & 250 & 1,712 & 1,514 & 448 & 343 & $\$ 16$ & 109 & 771 & 1,700 & $17, \operatorname{tg} 2$ & -541 \\
\hline 1904 & iB? & 1,873 & 1,346 & 714 & 916 & BA & 111 & 481 & 1,697 & 15.600 & -492 \\
\hline 1996 & 117 & 1,521 & 785 & 673 & 434 & 114 & 330 & 1 & 1,073 & 16,77 & +81 \\
\hline 1986 & 172 & 1,654 & 926 & 000 & 479 & 115 & 141 & 圽6 & 1.683 & 16,743 & -20 \\
\hline 1997 & 514 & $1,72 \overline{4}$ & 1,028 & 1,209 & 459 & 520 & 119 & 1,098 & 1605 & 17,335 & +642 \\
\hline
\end{tabular}

a Includes operator reported corrections for the years 1978 through 1981 . After 1981 operalors included conections with ravislons.

Detisions and adustiments = Col. $1+$ Col. 2 - Col. 3.

Total discoveries $=$ Col. $5+$ Cot. $6+$ Col. 7 .

Prowed reserves a Col. 10 irom prlor year + Col. 4 + Col. 8 - Col. 9 .

Based on following yoar data only.

fConsists conly' of operator reportod corrections and no othor adjuztriants.

$\vec{*}=$ Nib applicable.

Notes: Old means dlscovered th a prior year. New means dlscovered during the meprt year. The production estimales In this table are

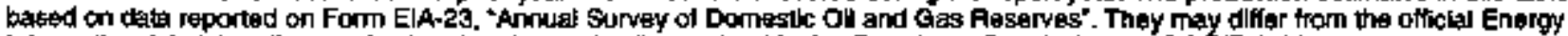

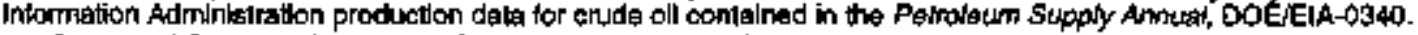

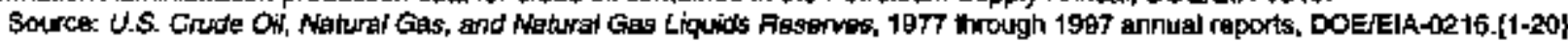


Table D3. U.S. Proved Reterves of Dry Natural Gas, 1976-1997 (Billion Cubic Feet at 14.73 psia and $60^{\circ}$ Fahrenheit)

\begin{tabular}{|c|c|c|c|c|c|c|c|c|c|c|c|}
\hline rear & (1) & 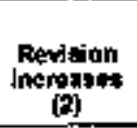 & $\begin{array}{c}\text { Reviaion } \\
\text { Decretiots: } \\
\text { (अ) }\end{array}$ & $\begin{array}{l}\text { Agulglons } \\
\text { and } \\
\text { adjusiments } \\
\text { [d] }\end{array}$ & $\begin{array}{c}\text { Extengiont } \\
\text { (디 }\end{array}$ & $\begin{array}{c}\text { New Fiald } \\
\text { Dleconverlet } \\
\text { (6) }\end{array}$ & 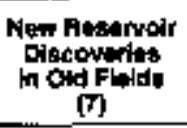 & $\begin{array}{c}\text { Totalt } \\
\text { Disebvited } \\
\text { (a) }\end{array}$ & $\begin{array}{c}\text { Production } \\
\text { (ө) }\end{array}$ & $\begin{array}{l}\text { Prowed } \\
\text { Restrves } \\
12191 \\
\text { (10) }\end{array}$ & $\begin{array}{l}\text { Chenge } \\
\text { drom } \\
\text { Priot Yeer } \\
\text { (11) }\end{array}$ \\
\hline 1976 & - & - & - & - & - & - & - & - & $\neg$ & 실. & $=$ \\
\hline 1977 & -20 & 13, f $^{\prime} 1$ & 15,296 & $-1,035$ & 9,129 & 3.173 & 3,301 & 14,603 & 18,643 & 207,413 & $-5,865$ \\
\hline 1978 & 2,429 & 14,969 & 15,904 & 1,404 & 9,582 & 3,860 & 4,579 & 18,021 & 18,905 & 206033 & +620 \\
\hline 1979 & $-2,264$ & 16,410 & 16,的9 & $-2,4 ; 03$ & 8,960 & 3,180 & 2.566 & 14,704 & 19,257 & 200,997 & $-7,0236$ \\
\hline 1980 & 1,201 & 16,972 & 15,923 & 2,250 & 9,357 & 2,539 & 2,577 & 14.473 & 19,699 & 199,021 & $-1,97 \theta$ \\
\hline 1981 & 1,627 & 侒A12 & 13,013 & 4.228 & 10,401 & 3,731 & 2.998 & 17,220 & 18,737 & 201,730 & +2.708 \\
\hline 1002 & 2.378 & 19,795 & 19.340 & $2,8,33$ & 8,349 & 2, ह97 & 3,419 & 14,456 & +7.500 & 201,512 & -210 \\
\hline 1980 & 9,000 & 17.602 & 17.017 & 3,075 & 6,908 & 1,574 & 2,985 & 11,448 & 15,769 & 200,247 & $-1,255$ \\
\hline 1804 & $-2,241$ & 17,841 & 14,712 & 869 & 8,299 & 2,536 & 2.686 & $13, \$ 21$ & 17.190 & 197,469 & $-2,784$ \\
\hline 1985 & $-1,708$ & 18,775 & 16,304 & 763 & 7.169 & 999 & 2.960 & 11,128 & 15,905 & 190,369 & $-4,094$ \\
\hline 1988 & 1,320 & 21.269 & 17.697 & 4,892 & 요, 0065 & 1,099 & $1.77 t$ & B. 0335 & 15,610 & 191,586 & -1.788 \\
\hline 1007 & 1,268 & 17,527 & 14,231 & 4.564 & 4,587 & 1,089 & 1,499 & 7,175 & 19,114 & 187211 & $-4,375$ \\
\hline 1908 & 2,190 & 30,367 & 30,427 & $-12,807$ & 0.003 & 1.636 & 1,900 & 10,350 & 18,670 & $9_{160,024}$ & -19.167 \\
\hline 1809 & 3,013 & 26,673 & 23,643 & 6,043 & 6.339 & 1,450 & 2,243 & 10,032 & 16,983 & 167.116 & -908 \\
\hline 1990 & 1,557 & t9,081 & 13,443 & 7,095 & 7,059 & 2,004 & 2,412 & 12,369 & 17,233 & 169,346 & $+2,230$ \\
\hline 1991 & 2,900 & $19, \mathbf{s} 90$ & 15,474 & 7,378 & 5.090 & 848 & 1.604 & 7,642 & 17,202 & 167.062 & -2.284 \\
\hline 1952 & 2.235 & 18,055 & 11,062 & 0.328 & 4,675 & Bd9 & 1.724 & 7,048 & 17,423 & 165,015 & $-2,047$ \\
\hline 1993 & 972 & 17,597 & 12,248 & 1,921 & 6,101 & 白穴 & 1,866 & $0,0 \approx 8$ & 17,789 & $\$ 62,415$ & $-2,6 \infty 0$ \\
\hline 1904 & 1.046 & $21, \$ 06$ & $15,8 \mathrm{~g} 1$ & 7,429 & 6,941 & 1,894 & 3,480 & 12315 & 10,322 & 163.837 & $+1,422$ \\
\hline 1985 & 580 & 20.465 & 12,731 & 8,314 & 6,843 & 1,665 & 2,452 & 10,561 & 17.906 & 165.148 & - 1.309 \\
\hline 1998 & 3,785 & 17,132 & 13,046 & 7,871 & 7.757 & 1,451 & 3,110 & 12,318 & 18,801 & 168,474 & $+1,328$ \\
\hline 196? & -590 & 21,658 & 16,755 & 4,912 & 10,585 & 2,861 & 2,392 & 15,648 & 19,211 & 167,223 & +749 \\
\hline
\end{tabular}

Indudeg operator reported corrections for the years 1978 threxph 1961 . Afler 1981 operators included corrections with revisions.

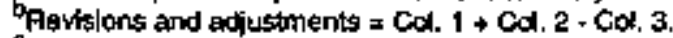

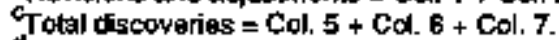

Proved reserves = Col. 10 irom pripr year + Col. 4 + Col. 8 - 130l. 9.

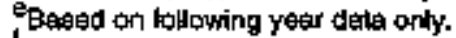

Consists onty ol operator reported corrections and on other adjustrments.

SAn unusually large revision decrease to North Siope dry nalural gas veserves was made in 1988. It recogrizes some 24.6 tillion cubic feet of downward revizions reporled during the last faw years by operalors because of econonic and markel conditions. ElA in previous years cerried these resense in the proved categony.

$-=$ Alot appilcable.

Notes: Old means discovered in a pror year. New means dlscovered during the report year. The production esfimates in thts tabse are

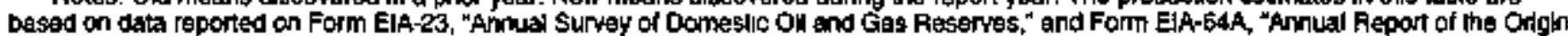
of Naturat Gas Llqubds Production". They may differ from the offletal Energy Imformallon Adoministration production data lor natural gas Comiatned In the Naturar Gas ANNuar, DOEEEIA-D137.

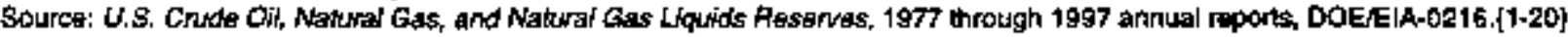


Table D4. U.S. Lower 48 Proved Reserves of Dry Natural Gas, 1976-1997 (Billion Cubic Feet at 14.73 psia and $60^{\circ}$ Fahrenheit)

\begin{tabular}{|c|c|c|c|c|c|c|c|c|c|c|c|}
\hline Yar & 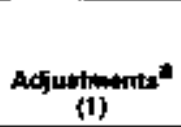 & $\begin{array}{l}\text { Arrision } \\
\text { Increagera } \\
\text { [2] }\end{array}$ & $\begin{array}{c}\text { Aowiolion } \\
\text { Derreapess } \\
\text { (a) }\end{array}$ & $\begin{array}{l}\text { Aeviations } \\
\text { and } \\
\text { Ackidualments } \\
\text { [4] }\end{array}$ & $\begin{array}{c}\text { Eximaiens } \\
\text { (5) }\end{array}$ & $\begin{array}{l}\text { New Fleqld } \\
\text { Driconeribs } \\
\text { (b) }\end{array}$ & $\begin{array}{c}\text { New Reservolr } \\
\text { Disogreries } \\
\text { in Old Finlds } \\
\text { (7) }\end{array}$ & $\begin{array}{c}\text { Totpe } \\
\text { Ditcoveries } \\
\text { (o) }\end{array}$ & $\begin{array}{l}\text { Production } \\
\text { (9] }\end{array}$ & $\begin{array}{l}\text { Proved } \\
\text { Raservet } \\
\text { 12/51 } \\
\text { (10) }\end{array}$ & $\begin{array}{l}\text { Chongh } \\
\text { trom } \\
\text { Prlor Your } \\
\text { (11) }\end{array}$ \\
\hline 1976 & $=$ & - & - & - & - & - & - & $=$ & - & $\mathrm{B}_{1 \mathrm{~B}, \mathrm{e}, \mathrm{e}}$ & - \\
\hline 1977 & $1-21$ & 13.699 & 15,229 & -1.561 & 8,056 & 3.173 & 3,301 & 14,530 & 18,637 & 175,170 & $-5,688$ \\
\hline 1978 & 2.446 & 13,912 & 14,670 & 1,689 & 9,582 & 3.880 & 4,277 & 17,719 & 18,589 & 175.968 & 618 \\
\hline 1979 & $-2,202$ & 15,691 & 16,$90 ;$ & $-2,009$ & 8,949 & 3,173 & 2,568 & $14,6 \mathrm{Bg}$ & 19,029 & 169.733 & $-7,240$ \\
\hline 1000 & 1,163 & 15,891 & 15,819 & 1,225 & 9,046 & 2,539 & $2,5 \pi$ & 14,162 & 18,486 & 166,639 & -4090 \\
\hline 1081 & $1,8 d 0$ & 10.258 & 13,752 & 4,340 & 10,485 & 3.731 & 2.994 & 17,210 & 18,502 & 168,693 & 3,054 \\
\hline 1902 & 2,367 & 17,570 & 19,318 & 619 & 8,349 & 2,687 & 3,419 & 14,455 & 17.245 & 166,522 & -2.171 \\
\hline 1083 & 3,080 & 17,296 & 16,875 & 3,510 & 6.908 & 1,574 & 2.965 & 11,447 & 15,515 & 165,064 & -558 \\
\hline 1994 & $-2,245$ & 16,934 & 14,317 & 372 & 0,298 & 2,536 & 2,686 & 13,520 & 16,869 & 1自2,9日7 & -2097 \\
\hline 1905 & -1.349 & 10,252 & 15,758 & 1,151 & 7,090 & 990 & 2,800 & 11,057 & 15,673 & 159,522 & $-3,458$ \\
\hline 1 的s & 1,自1自 & 21,024 & 16,940 & 5,782 & 6,084 & 1,099 & 1,761 & 8,924 & $15,2 \%$ & 158,622 & -600 \\
\hline 1987 & 1,086 & 16,8009 & 14.164 & 3,711 & 4,542 & $1,0 \mathrm{r}$ & 1,499 & 7,118 & 15,765 & 153,986 & -4.936 \\
\hline 1988 & 2.013 & 22,571 & 13,876 & 10.912 & 6,771 & 1.638 & 1,900 & 10,318 & 16,270 & 158.946 & 4.980 \\
\hline 1989 & 2,997 & 26,446 & 23,507 & 5,935 & 6,184 & 1,450 & 2,243 & 9,877 & 10,582 & 158,177 & -7放 \\
\hline 1990 & †.677 & 17,016 & 13,344 & 6,449 & $7, \mathrm{~B}$ & 2,004 & 2,412 & 12,314 & $+6,804$ & 160,048 & +1869 \\
\hline 1091 & 2,067 & 19,095 & 15,235 & 6,827 & 5,074 & 848 & 1,563 & 7,485 & 16,849 & 157,509 & $-2,537$ \\
\hline 1992 & 1,940 & โ7. Btr & $1 t, 941$ & $7, \mathrm{Bg}+3$ & 4,621 & 649 & 1,724 & 6,904 & 17,009 & 155,377 & $-2,132$ \\
\hline 1993 & 915 & 16,918 & 12,139 & 5,694 & 6,076 & 899 & 1,858 & 8,833 & 17,396 & 152,500 & -2869 \\
\hline 1994 & 1,6506 & 21,121 & 15,832 & $7,1 \mathrm{BS}$ & 6.936 & 1, 自4 & 3,480 & 12,310 & 17,899 & 154,104 & +1596 \\
\hline 1995 & 973 & 19,900 & 12,680 & B, 196 & 6,801 & 1,866 & 2,452 & 10,918 & 17,570 & 155,648 & +1.545 \\
\hline 1985 & 3,640 & 16,830 & 12,075 & 7,695 & 7,751 & 1,390 & 3,110 & 12,251 & 19,415 & 157,180 & $+1,531$ \\
\hline 1097 & -609 & 19,849 & 16,657 & 2,583 & 10.571 & 2,681 & 2.382 & 15,634 & 18,734 & 156,661 & -519 \\
\hline
\end{tabular}

Includes operator reported comecticns for the years 1978 through 1981. Afler 1981 operalors inchuded corrections with revitions.

Bevisions and adjustrients $=$ Col. $1+$ Col. 2 - Col. 3 .

Total dlscoveries = Cor. $5+$ Col. 6 + Col. 7 .

Prowed reperves = Col, 10 from prior year + Col. $4+$ Cos. $8-$ Col. 9 .

Based on follawing year data only.

Conststs only of operalor reported corrections and no olther adjustments.

- = Nor applicable.

Notes: Old means discovered in a prior year. Naw moans discovered cturing the roport year. The produltion tsitmates in lhis bable are

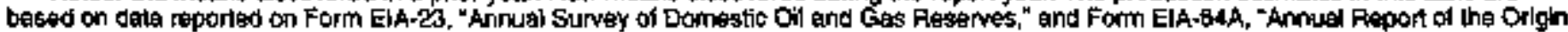
of Nasural Bas Liqulds Produxtlon". They miey differ trom the olficial Energy Information Adrininstralon production data for natural gas conteingd in the Netorar Gas AnNUar, DÓEJEIA-0131.

Source: U.S. Crade ON, Natura' Gas, and Nahwat Gas Liquids Regerves, 1977 hrough 1997 annual reports, DOEFEIA-02 16.[1-20] 
Table 05. U.S. Proved Reserves of Wet Natural Gas, After Lease Separatlon, 1978-1997 (Billion Cubic Feet al 14.73 psia and $60^{\circ}$ Fahrenheit)

\begin{tabular}{|c|c|c|c|c|c|c|c|c|c|c|c|}
\hline var & Ad]urtmantes & 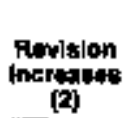 & $\begin{array}{c}\text { Roviaion } \\
\text { Dererangats } \\
\text { (J) }\end{array}$ & $\begin{array}{c}\text { Auvitions } \\
\text { and } \\
\text { Adfititmento } \\
\text { (4) }\end{array}$ & $\begin{array}{c}\text { Extengions } \\
\text { (5) }\end{array}$ & 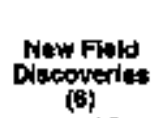 & 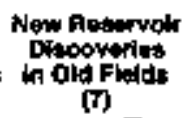 & $\begin{array}{c}\text { Total" } \\
\text { Dlecowerive } \\
\text { (8) }\end{array}$ & $\begin{array}{l}\text { Prostupton } \\
\text { (D) }\end{array}$ & 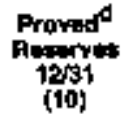 & $\begin{array}{l}\text { Change } \\
\text { from } \\
\text { Prior Yor } \\
\text { [t1] }\end{array}$ \\
\hline 1978 & - & - & - & - & - & - & - & - & - & ${ }_{2008,033}$ & - \\
\hline 197g & 5,358 & 17,077 & 17.300 & 5,133 & 9,3 ב & 3.279 & 2637 & 15,248 & 20,079 & 208,335 & +302 \\
\hline 1980 & 1,250 & 17.688 & $16,53 \mathrm{r}$ & 2,390 & 9,757 & 2,629 & 2,618 & 15,004 & 19,500 & 206.250 & -2.076 \\
\hline 1981 & 2,057 & 17,158 & 14,413 & 4,800 & 10.979 & 3,670 & 3,000 & 17,929 & 15,554 & 200,434 & $+3,175$ \\
\hline 1992 & 2.5peg & 20.598 & 20,141 & 3,053 & 8,754 & 2,785 & 3.520 & 15,059 & 18,292 & 20,254 & -160 \\
\hline 1983 & $4,3 \times \sqrt{3}$ & 19.442 & 18,385 & 4,420 & 7,203 & 1,629 & 3,071 & 11,902 & 16,590 & 209,046 & -206 \\
\hline 1984 & $-2,413$ & 18,751 & 15,418 & 920 & $8,6 \mathrm{BP}$ & 2,584 & 2,778 & 14,050 & 18,698 & 205,394 & $-3,0$ 하 \\
\hline 1985 & $-1,299$ & 10,732 & 17,045 & 1,308 & $7.6,35$ & 1,040 & 3.063 & 11,自安 & 15,7嫜 & 202,202 & $-3,782$ \\
\hline 1998 & 2,137 & 22,392 & 18,557 & 5.972 & 6,359 & 1,122 & 1,855 & 9,338 & 16,101 & 201,100 & $-1,003$ \\
\hline $19 d \vec{t}$ & 1,190 & 18,455 & 14.933 & 4.721 & 4,812 & 1.128 & 1.556 & 7,502 & 16,904 & 196,429 & -4.681 \\
\hline 1898 & 2,180 & 24,639 & $I_{39,589}$ & $-12,751$ & 7,132 & 1,677 & 1.979 & 10,788 & 17,466 & $I_{176,899}$ & $-19,428$ \\
\hline 1999 & 2,537 & 27,044 & 24,624 & 5,757 & 6,623 & 1,489 & 2.313 & 10,424 & 17,758 & 175,428 & $-1,571$ \\
\hline 1960 & 1.494 & 19,061 & 14,024 & 7,391 & 9,287 & 2,041 & 2,492 & 12,820 & 18,003 & 177.576 & +2.148 \\
\hline 1991 & 3,398 & 20,758 & 16.189 & 7,937 & 5,296 & 871 & 1,655 & 7, 名류) & 18,012 & 176,325 & -2.251 \\
\hline 1992 & 2,543 & $1 \mathrm{~B}, 906$ & 12,532 & B.917 & 4,095 & 688 & 1,773 & 7,338 & 18,269 & 173,309 & $-2,016$ \\
\hline 1903 & 1,048 & 16,394 & 12.853 & 6.599 & $6,37 \mathrm{~F}$ & $92 ?$ & 1,930 & 0,233 & 18,641 & 170,490 & $-2,819$ \\
\hline 1994 & 1,977 & 22,343 & 16,509 & 7,813 & 7,295 & 1,941 & 3,606 & $t 2,840$ & 19,210 & +71.939 & $+1,449$ \\
\hline 1005 & 889 & 21.640 & 19,467 & $\theta, 000$ & $7,20 \mathrm{~d}$ & 1,700 & 2518 & $11,43 y$ & 18,974 & 173,476 & $+1,537$ \\
\hline 1996 & 4,2089 & $18,0<4$ & 13.757 & B.565 & 9.155 & 1,491 & 3.209 & $12,8=9$ & 19,7es & 175,147 & +1.871 \\
\hline 1997 & -730 & 22,712 & 17.655 & 4.327 & 11,175 & 2.747 & 2,456 & 16,381 & 30,134 & 175,721 & +574 \\
\hline
\end{tabular}

Indudes operalar jeported corrections for the years 1978 through 1981 . Alter 1981 operalors inchucted contections with revisions.

"Pevisions and adjustments $=\mathrm{Col}, 1+\mathrm{Col}, 2$ - Col. 3 .

TTotal discoveriles $=$ Col. $5+$ Coll. $6+$ Col. 7 .

dproved reserves = Col. 10 fróm prior yér + Col. $4+$ Col. B - Col. 9.

Eased on following year dala ondy.

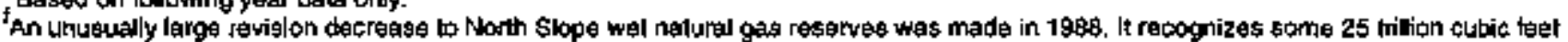
of dowmward revisions reported duaing the last few years by operators because of economic and market conditions. ElA in previbus years carrled these reserves in the proved category.

- - Not applicable.

Notes: Old maans discovered in a prlor year. New means diccovered during the report year. The production estimales in lhis table are

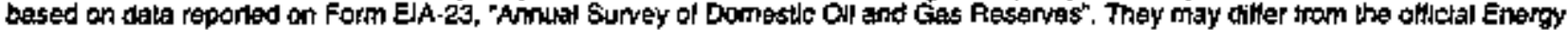
Intermation Adrinistretion production dala for nalural gas contained in the Nakural Gas Anfust, DOEJEIA-013.

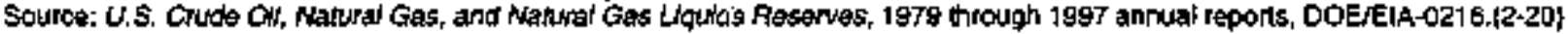


Table D6. U.S. Lower 48 Proved Reserves of Wet Natural Gas, After Lease Separation, 1978-1997 (Billion Cubic Feel at 14.73 psia and $60^{\circ}$ Fahrenheit)

\begin{tabular}{|c|c|c|c|c|c|c|c|c|c|c|c|}
\hline Yar & Aciuntmonts: & 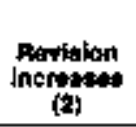 & $\begin{array}{c}\text { Pewiblan } \\
\text { Dectiaten } \\
\text { (3) }\end{array}$ & 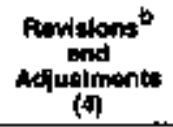 & $\begin{array}{c}\text { Extonofions } \\
\text { (5) }\end{array}$ & $\begin{array}{c}\text { How Fiald } \\
\text { Dleconteries } \\
\text { (9) }\end{array}$ & 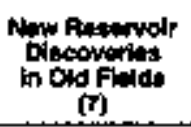 & $\begin{array}{c}\text { Total" } \\
\text { Dlecoveriot } \\
\text { (t) }\end{array}$ & $\begin{array}{c}\text { Produolion } \\
(\hat{(\theta)})\end{array}$ & $\begin{array}{l}\text { Prowed } \\
\text { Pesenven } \\
\text { 1201 } \\
(10)\end{array}$ & $\begin{array}{c}\text { Chamge } \\
\text { 1rom } \\
\text { Prtor Your } \\
\text { (11) } \\
\end{array}$ \\
\hline 1978 & - & - & - & - & - & - & - & - & - & $0_{175,080}$ & - \\
\hline 1979 & 5,402 & 16,358 & 17.0009 & 4,691 & 9,391 & 3.264 & 2,637 & 15,232 & 19,651 & 178,000 & +72 \\
\hline 1000 & 1,218 & 16,577 & 16,427 & 1,368 & 9,446 & 2,029 & 2,640 & 14,723 & $19,20 ?$ & 172.664 & $-3,196$ \\
\hline 1981 & 2,270 & 17,002 & 14.952 & 4.920 & 10,973 & 3,870 & 3,076 & 17,919 & 79,319 & 176,385 & $\bullet 3,521$ \\
\hline 1982 & Z & 18.371 & 20,110 & 938 & 8,754 & 2,385 & 3,520 & 15.059 & 18,000 & 174,252 & $-2,133$ \\
\hline 1983 & 4,3366 & 18,138 & 17,843 & 4,859 & 7,202 & $1,62 \mathrm{~B}$ & 3,071 & 11,961 & $16,31\}$ & 174,755 & +5003 \\
\hline 1884 & $-2,409$ & 17.844 & 15,023 & 412 & 8,687 & 2,584 & $2,77 \theta$ & 14.049 & 17,700 & 171,508 & $-3,247$ \\
\hline 1985 & $-1,313$ & 19,200 & 16,490 & 1,400 & 7,483 & 1,040 & 3,053 & 11,558 & 16,485 & $16\}, 97 \theta$ & $-3,529$ \\
\hline 1968 & 2,114 & 22,207 & 17,797 & 0,524 & 6.357 & $1,1 \mathrm{~g} 7$ & 1,645 & 9,324 & 16,073 & 167,754 & -225 \\
\hline 1987 & 1,200 & 17,793 & 14,865 & 4,068 & 4.772 & 1,116 & $1,5 \$ 6$ & 7,444 & 16,653 & $1 \mathrm{e2}, 71 \mathrm{~s}$ & $-6,041$ \\
\hline 1988 & 2,095 & 23,029 & 14,439 & 17,415 & 7,099 & 1,677 & 1,979 & 10,755 & 17,060 & 163,820 & $+5,107$ \\
\hline $18 B$ & 2545 & 27,616 & $24,48 B$ & 5,673 & 6,467 & 1.485 & 2.313 & 10.365 & 17,340 & 168,409 & $-1.41 t$ \\
\hline 1990 & $\dagger, 811$ & 18,784 & 13,925 & 6,670 & B.2352 & 2,041 & 2,492 & 12,765 & 17,601 & 169,183 & $+1,774$ \\
\hline 1991 & 3,367 & 79,861 & 15,346 & 3,300 & $5,2 B 1$ & 871 & 1,614 & 7.766 & 17,655 & 165,672 & $-2,511$ \\
\hline 1992 & 2,296 & 78,728 & 12,511 & 8,492 & 4.840 & 608 & 1,773 & 7,281 & 17,861 & 163,584 & $-2,080$ \\
\hline t593 & 996 & 17,714 & 12,749 & 5,967 & 6,349 & 927 & 1,922 & 9,198 & 18,245 & 180,504 & $-3,000$ \\
\hline t984 & 1.924 & 22,071 & 16,458 & 7,537 & 7.294 & 1,941 & 3,600 & $12,84^{\dagger}$ & 19.756 & 162,126 & $+1,622$ \\
\hline 1995 & 1,304 & 20,928 & 13,403 & 8,629 & 7,182 & 1,300 & 2,518 & 11,309 & 18,443 & 103,901 & $\bullet 1,775$ \\
\hline 1908 & 4,219 & 17,632 & 13,589 & a,465 & 0,183 & 1,430 & 3,203 & 12,902 & 19.937 & 165,051 & $+1,950$ \\
\hline 1997 & -836 & 20,878 & 17,656 & 2,497 & 11,165 & 2,747 & 2.455 & 16,367 & 19,867 & 106,048 & $-\operatorname{exs}$ \\
\hline
\end{tabular}

Inchudes operater reported corrections for the years 1970 through 19e1. After 1981 operators included corrections with revlsions

TRevisions and ad|usturents = Cod, 1 + Col. 2 - Col. 3.

${ }^{\circ}$ Tolen discoreries $=\mathrm{C}$ ol. $5+\mathrm{Col} .6+\mathrm{Col} .7$.

Trowed reserves $=$ Col. 10 1rom prlor year + Col, $4+$ Col $.8 \cdot$ Col 9.

"Based on lodlowing year data only.

- Not applicable.

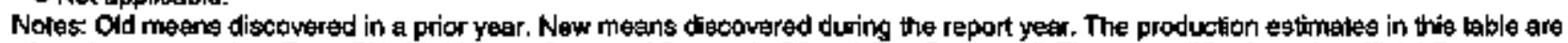
based on data reported on Form ElA.ż, "Annual Survey of Domestic Onl and Gas Roserves". They may difter from the otticial Energy'

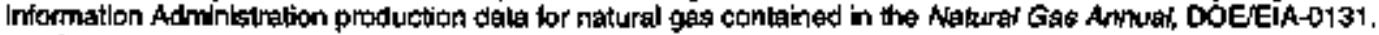

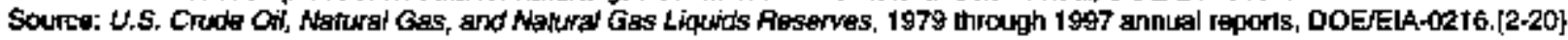


Table D7. U.S. Proved Reserves of Natural Gas Liquids, 1978-1997 (Million Barrels of 42 U.S. Gallons)

\begin{tabular}{|c|c|c|c|c|c|c|c|c|c|c|c|}
\hline Yeer & $\underset{\text { (t) }}{\text { Ac|usfimints }}$ & 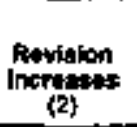 & 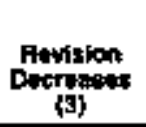 & 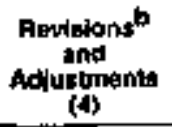 & $\begin{array}{c}\text { Extentionts } \\
\text { (5) }\end{array}$ & $\begin{array}{l}\text { New Figld } \\
\text { Oloctoviteríat } \\
\text { (B) }\end{array}$ & 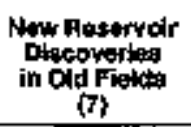 & $\begin{array}{c}\text { Totalc } \\
\text { Diacowerias } \\
\text { (8) }\end{array}$ & $\begin{array}{c}\text { Prothertinn } \\
\text { [9] }\end{array}$ & $\begin{array}{l}\text { Proredt } \\
\text { Regerves } \\
1231 \\
\text { (10) }\end{array}$ & $\begin{array}{l}\text { Ehangs } \\
\text { fram } \\
\text { Prich Yoe } \\
\text { (11) }\end{array}$ \\
\hline 1978 & - & - & - & $=$ & - & - & - & - & $=$ & $0_{6,772}$ & $=$ \\
\hline 1979 & $f_{k 4}$ & 67 & 726 & 15 & 354 & 94 & $\theta 7$ & s\$s & 727 & $6,6 \$ 5$ & -157 \\
\hline 1900 & 153 & 743 & 649 & 257 & $42 \xi_{1}$ & 90 & 79 & 587 & $7 \$ 1$ & 6,729 & 4123 \\
\hline 198: & 234 & 729 & 643 & 317 & $54 i$ & 131 & 91 & 784 & 741 & 7,068 & +340 \\
\hline 1982 & 299 & 911 & 832 & 278 & $37 t$ & 112 & 100 & $5 S 9$ & 721 & 7,221 & +159 \\
\hline 1963 & 649 & 847 & 781 & 915 & 31 & 70 & 99 & 490 & 725 & 7,801 & +890 \\
\hline 18P4 & -123 & 86 & 724 & 19 & 34: & 55 & 96 & 198 & 776 & 7,043 & -258 \\
\hline $19 \$ 5$ & 426 & 906 & 744 & 580 & $33 \mathrm{H}^{\prime}$ & 44 & 85 & 486 & 753 & 7,944 & +301 \\
\hline 1986 & 307 & 1,030 & 87 & 590 & 265 & 34 & 72 & 369 & 738 & 6.165 & +221 \\
\hline 1097 & 231 & 847 & 656 & 422 & $21 \hat{i}$ & 39 & 55 & 307 & 747 & B.147 & -18 \\
\hline 19888 & 11 & 1,168 & 715 & 464 & 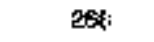 & $\$ 1$ & 72 & 9日81 & 754 & 8,238 & 491 \\
\hline 1390 & -277 & 1,143 & 1,000 & -154 & $25+1$ & B3 & 74 & 416 & 731 & 7,760 & -469 \\
\hline 1990 & - & B27 & $\theta 0 \theta$ & 198 & $2 \theta: 1$ & 39 & 73 & 111 & 732 & 7,506 & -183 \\
\hline 1981 & 233 & 625 & 695 & 363 & $18 \varsubsetneqq$ & 25 & 55 & 269 & 754 & 7,464 & -122 \\
\hline+992 & 225 & 00 & 345 & 488 & 190. & 20 & $B A$ & 274 & 773 & 7.459 & -13 \\
\hline 1993 & 102 & 704 & 640 & 226 & $24 \mathrm{Bi}$ & 24 & EA & 333 & T珀 & 3,222 & -229 \\
\hline 1994 & 43 & B73 & 676 & 240 & $\mathbf{\$} 1 t$. & S4 & 131 & 490 & 791 & 7.170 & -52 \\
\hline 196 & 182 & 时白 & 691 & 469 & 43:! & 52 & 67 & 551 & 791 & 7,390 & +220 \\
\hline$t 996$ & 474 & B44 & 69 & 649 & $45^{\prime}$ & 65 & 109 & 623 & 850 & 7.823 & +424 \\
\hline 1997 & -14 & $1,+198$ & 910 & 275 & $595 i$ & 114 & 90 & 739 & 864 & 7,973 & +150 \\
\hline
\end{tabular}

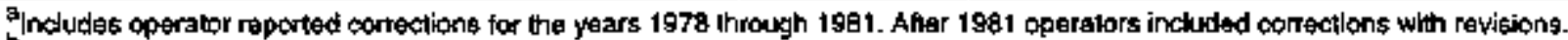

b Pevisions end adjustments = Col. $1+$ Cod. 2 - Col. 3.

Total dlscovertes = Col. 5 + Col. 6 + Col. 7.

Troved reserves = Col. 10 from prior year + Col, 4 + Coll $B$ - Col. 9.

Based on following year dala only.

fConsists only of operaker raportad corrections and no other adjustrmenls.

$-=$ Not applichble.

Notes: Old means dscowered in a prior year. New meens chiscovered during the report year. The production estimates in this table are

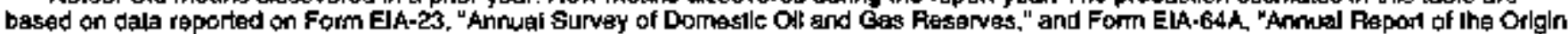
of Natural Gas Liqu|ds Production", They may differ trom the offic|al Energy informathon Adm|nistrathon production date Ior nalurat gas lichuids conteined in the Nalural Ges Annuat, DOENElA-0131.

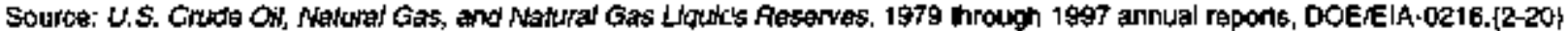


Table D8. U.S. Lower 48 Proved Roseryes of Notural Gas Liquids, 1978-1997 (Million Barrels of 42 U.S. Gallons)

\begin{tabular}{|c|c|c|c|c|c|c|c|c|c|c|c|}
\hline Yere & Ad] & 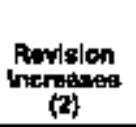 & 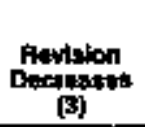 & $\begin{array}{l}\text { Fuvialonsts } \\
\text { and } \\
\text { Autivitiments } \\
\text { (d) }\end{array}$ & $\begin{array}{c}\text { Exidertolions } \\
\text { (15) }\end{array}$ & $\begin{array}{c}\text { New Fistd } \\
\text { Discontulad } \\
\text { [(\$) }\end{array}$ & $\begin{array}{l}\text { Dieconedes } \\
\text { in Old Fiether } \\
\text { (T) }\end{array}$ & 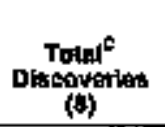 & $\begin{array}{c}\text { Prthortion } \\
\text { [9] }\end{array}$ & 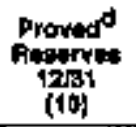 & $\begin{array}{l}\text { Chinge } \\
\text { from } \\
\text { Pritr Year } \\
\text { (11) }\end{array}$ \\
\hline 1978 & - & - & - & - & $=$ & - & - & - & - & $e_{6,749}$ & - \\
\hline 1979 & $f_{69}$ & 677 & 726 & 14 & 304 & 9 & 97 & 555 & 726 & 6,592 & -157 \\
\hline 1980 & 165 & 743 & 639 & 269 & 418 & 90 & 79 & 587 & 731 & 6,717 & +125 \\
\hline 1991 & 230 & 720 & f43 & 378 & 542 & 131 & 91 & 764 & 741 & 7,058 & +341 \\
\hline $19 a 2$ & 300 & B11 & 832 & 279 & 375 & 112 & tos & 596 & 721 & 7,212 & +154 \\
\hline 18的 & 850 & B47 & 781 & 916 & 321 & 70 & 99 & 490 & 725 & 7,898 & $+6 B 1$ \\
\hline 1904 & -115 & $B 47$ & 724 & 8 & 348 & 55 & 88 & 499 & 776 & 7,624 & -269 \\
\hline 1 영다. & 70 & gosg & 731 & $2 \not 20$ & 34 & 4 & 25 & 463 & 748 & 7,561 & -6 \\
\hline 1986 & 353 & 1,020 & $B 04$ & 580 & 283 & 34 & 72 & 369 & 735 & 7,784 & +223 \\
\hline 1987 & 170 & 940 & 655 & 370 & 212 & 39 & 55 & 906 & 7951 & 7.729 & -55 \\
\hline 1988 & 10 & $1,+67$ & 715 & 482 & 287 & 41 & 72 & 380 & 734 & 7,837 & +100 \\
\hline $18 B 9$ & -273 & 1,141 & 1.018 & -150 & 258 & $B 3$ & 74 & 418 & 714 & 7,389 & -448 \\
\hline 1800 & $-\infty 0$ & 县7 & $\infty 8$ & โ61 & 298 & 39 & 73 & 410 & $7+4$ & 7.246 & -143 \\
\hline 1991 & 183 & 815 & 677 & 321 & 187 & 25 & 55 & 267 & 730 & 7,104 & -142 \\
\hline 1002 & 295 & 796 & 542 & 479 & 183 & 20 & 6d & 267 & 746 & 7,104 & o \\
\hline 1993 & to1 & 755 & 631 & 225 & 245 & 24 & 白 & 353 & 781 & 169,6 & 1012 \\
\hline 1994 & 38 & 973 & 678 & 234 & 314 & 54 & 131 & 499 & 765 & 8,809 & -39 \\
\hline 1095 & $20 \mathrm{~d}$ & 916 & 68 & 434 & $43 \underset{2}{ }$ & 59 & $\theta 7$ & 551 & 761 & 7,009 & +224 \\
\hline 1998 & 417 & 032 & 654 & 595 & 450 & $\$ 6$ & 109 & 615 & 817 & 7,488 & +393 \\
\hline 1907 & $-t 07$ & 965 & 910 & -52 & 533 & 114 & 㫙 & 737 & 629 & 7,342 & -144 \\
\hline
\end{tabular}

Includes operater reporied correations for the years 1978 through 1981. After 1981 operators inchuded corrections with revisions.

Bevisions and adjustments = Cor. $1+$ Col, 2 - Col. 3

Total dlscoveries a Col. $5+$ Col. $6+$ Col. 7 .

dProwed reserves = Col. 10 from pror year $+\operatorname{Cot} 4+$ Col. $8-$ Cod. 9 .

Based on lollowing yedor data conly.

'Consists only of operator reported corrections and no other adjustments.

- = Not applyabla.

Notes: Old moans discovered in a prior year. New means figcowered duing the report yeer. The production estimates in this table are besed on data reported on Form EIA-23, "Annual Survey of Domestic Oi and Ges Reserves," and Form ElA-64A, "Annual Feport of the Origin

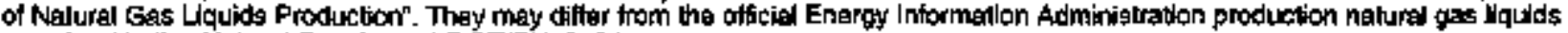
conteinad in the Netwat Gas Arrid, DOEFEIA-0131.

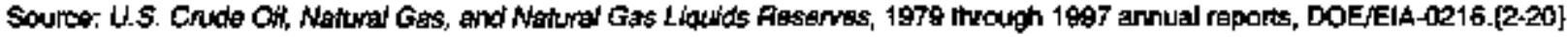

Energy Informalion Adminiotration 
Table D9. Deepwater Production and Proved Reserves of the Gulf of Mexico Federal Offshore, 1992-1997

\begin{tabular}{|c|c|c|c|c|c|c|}
\hline \multirow[b]{2}{*}{ Year } & \multicolumn{3}{|c|}{ Gulf of hexico. } & \multicolumn{2}{|c|}{ Depth } & \multirow[b]{2}{*}{$\begin{array}{l}\text { Deepwater } \\
\text { Percentege }\end{array}$} \\
\hline & Total & Louliaiena" & Toxas & $\begin{array}{l}\text { Greeter than } \\
200 \text { meters }\end{array}$ & $\begin{array}{l}\text { Lese then } \\
200 \text { motars }\end{array}$ & \\
\hline & \multicolumn{5}{|c|}{ Crude Oll (miltion barrels of 42 U.S. gallons) } & \\
\hline Production & & & & & & \\
\hline 1992 & 267 & 253 & 14 & R48 & R221 & $\mathbf{R} 17.2$ \\
\hline 1993 & 266 & 252 & 14 & R46 & $R 220$ & R17.9 \\
\hline 1994 & 265 & 245 & 20 & P53 & R212 & $\mathrm{R} 20.1$ \\
\hline 1995 & 292 & 262 & 30 & A77 & Re15 & R26.4 \\
\hline 1996 & 303 & 265 & 38 & $\mathrm{R} 90$ & R213 & R29.7 \\
\hline 1997 & 342 & 298 & 44 & 123 & 219 & 36.0 \\
\hline \multicolumn{7}{|l|}{ Ponervon } \\
\hline 1892 & 1,835 & 1,643 & 192 & A557 & $\mathrm{F} 1,279$ & R3 30.4 \\
\hline 1993 & 2,072 & 1,880 & +92 & $\mathrm{Ra24}$ & A1,248 & $R 30.8$ \\
\hline 1994 & 2,127 & 1,922 & 205 & $\mathrm{~F} 877$ & $R 1,250$ & R41.2 \\
\hline 1995 & 2,518 & 2,269 & 249 & A1,241 & R1,277 & $\mathrm{R} 49.3$ \\
\hline 1096 & 2,567 & $\hat{2}, 357$ & 210 & P1,311 & R1,258 & R51.1 \\
\hline 1997 & 2,949 & 2,587 & 362 & 1,682 & 1,267 & 57.0 \\
\hline
\end{tabular}

Natumal Gas, Wot After Lease Separation

\begin{tabular}{|c|c|c|c|c|c|c|}
\hline \multirow{2}{*}{ Production } & \multicolumn{5}{|c|}{ (billion cubic foet et $14.73 \mathrm{psia}$ and $60^{\circ}$ Fahrenheit) } & \multirow{3}{*}{ R3.6 } \\
\hline & & & & & & \\
\hline 1992 & 4,576 & 3,292 & :284 & A166 & $\mathbf{R} \mathbf{4 , 4 1 0}$ & \\
\hline 1993 & 4,651 & 3,383 & -.268 & ค229 & R4,422 & R4.9 \\
\hline 1894 & 4,797 & 3,505 & 1,292 & F294 & $\mathbf{R} 4,503$ & R6. 1 \\
\hline 1995 & 4,679 & 3,421 & I,258 & R3554 & R4,315 & A7.8 \\
\hline 1996 & 5,046 & 3,752 & 1,293 & R.549 & $\mathbf{F} 4,496$ & F10.9 \\
\hline 1997 & 5,290 & 3,984 & 1.240 & 577 & 4,653 & 11.0 \\
\hline \multicolumn{7}{|l|}{ Reserves } \\
\hline 1092 & 27,050 & 20,008 & $r, 044$ & R3,273 & $\mathrm{A} 23, \pi / 7$ & F12.1 \\
\hline 1990 & 26,463 & 19,751 & $\$, 712$ & $\mathbf{R 3 , 4 9 5}$ & A22,968 & R13.2 \\
\hline 4994 & 27,626 & 21,208 & $1 ;, 418$ & $\mathbf{R}, \pi / 2$ & F22,854 & F17.3 \\
\hline 1995 & 28,229 & 21,664 & 1,566 & R5,811 & R22,418 & R20.6 \\
\hline 1996 & 28,153 & 22,119 & 13,0304 & P6,389 & $\mathbf{R} 21,7 \mathbf{6} \mathbf{4}$ & R22.7 7 \\
\hline \multirow[t]{2}{*}{1997} & 28.455 & 22,428 & 6,027 & 7,491 & $20,95,4$ & 26.3 \\
\hline & \multicolumn{5}{|c|}{ Naturtu Gas Liquids (million barrele of 42 U.S. gallons) } & \\
\hline \multicolumn{7}{|l|}{ Production } \\
\hline 1992 & $\$ 1$ & 76 & 15 & R4 & R87 & $\mathbf{F 4 , 4}$ \\
\hline 1993 & 97 & 80 & 17 & Fo6 & F91 & R日.2 \\
\hline 1994 & 98 & 89 & 16 & A6 & $\mathbf{P} \theta \mathbf{2}$ & A6.1 \\
\hline 1995 & 85 & 71 & 14 & $\mathbf{R} 12$ & A7t & R14.1 \\
\hline 1996 & 101 & B4 & 17 & $R+3$ & A白高 & R12.,9 \\
\hline 1947 & 140 & 123 & 17 & 17 & 123 & 121 \\
\hline \multicolumn{7}{|l|}{ Reserves } \\
\hline 1992 & 590 & 472 & 118 & R91 & R499 & R15.4 \\
\hline 1993 & $\mathrm{BDS}$ & 490 & 115 & $R \otimes 7$ & P508 & R16.0 \\
\hline 1994 & 603 & 500 & 103 & R110 & R493 & R18.2 \\
\hline 1995 & 630 & 496 & 134 & R2O4 & R336 & R46.7 \\
\hline 1998 & 753 & 621 & 132 & $\mathrm{R} 300$ & R453 & F.39.8 \\
\hline 1997 & 906 & 785 & 121 & 349 & 557 & 38.5 \\
\hline
\end{tabular}

ancludes Federal Offthore Alabauma.

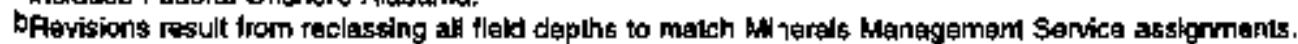

Source; Based on dab reported on Form EIA-23, "Anmuad Strvey of Domestbc Oll and Gas Aesarves." 
Appendix $E$

Summary of Data Collection Operations 



\section{Summary of Data Collection Operations}

\section{Form ElA-23 Survey Design}

The data collected on Forn ELA-23, "Annual Survey of Domestic Oil and Gas Reserves," were used to produce this report. This section provides information concerning the survey design, response statistics, reporting requirements, and frame maintenance.

Form EIA-23 is mailed arnually to all known large and intermediate size operators, and a scientífically selected sample of small operators. Operator size categories were based upon their anuual production as indicated in various Federal, State, and commercial records. The term State/subdivision refers to an individual subdivisjion within a State or an individual State that is not subdivided. Operators were divided into the three size categories shown below.

- Category I - Large Operators: Operators who produced 1.5 million batrels or more of crude oil, or 15 billion cubic feet or more of natural gas, or both.

- Category Il - Intemediate Operators: Operators who produced at least 400,000 barrels of crude oil or 2 billion cubic feet of natural gas, or both, but less than Category I operators.

- Category IIl - Small Operators: Operators who produced less than the Category if operators.

Category III operators were further subdivided into operators sampled with Certainty (Certainty) and operators that were randomly sampled (Noncertainty).

Data were filed for calendar year 1997 by crude oil or natural gas well operators who weze active as of December 31, 1997. ELA defines an operator as an organization or person responsible for the management and day-to-day operation of crude oil or natural gas wells. The purpose of this definition is to eliminate responses from royalty owners, working interest owners (unless they are also operators), and others not directly responsible for operations. An operator need not be a separately incorporated entity. To minimize reporting burden, corporations are permitted to report on the basis of operating units of the company converient for them. A large corporation may be represented by a single form or by several forms.

Table E1 shows a comparison of the ELA-23 sample and sampling frame between 1990 and 1997, and depicts the number of active operators, 1990 showing the largest in the series. The 1997 sampling frame consisted of 180 Category It 461 Category II, 1,194 Category III Certainty, and 20,843 Category III Noncertainty operators, for a total of 22,678 active operators. The survey sample consisted of 1,835 operators selected with certainty that included all of the Category I and II Certainty operators, the 1,194 smaller operators that were selected with certainty because of their size in relation to the area or areas in which they operated, and 1,645 Noncertainty operators selected as a systematic random sample of the remaining operators.

\section{Form EIA-23 Response Statistics}

Each company and its parent company or subsidiaries were required to file Form ELA-23 if they met the survey specifications. Response to the 1997 survey is summarized in Table E2. EIA makes a considerable effort to gain responses from all operators. About 13 percent of those selected turned out to be nonoperators (those that reported being nonoperators during the report year and operators that could not be located). Of the $\mathbf{4 4 2}$ nonoperators, $\mathbf{4 3}$ had successor operators that had taken over the production of the nonoperator. These successor operators were subsequently sampled. The overall response tate for the 1997 survey was 99.8 percent. This compares with a 100 percent overall response rate for all operators in 1996.

\section{Form EIA-23 Reporting Requirements}

The collection format for Form EIA-23 actually consists of two forms. The form the respondent is required to file is dependent upon the annual production levels of crude oil, natural gas, and lease condensate. Category I and Category Il operators tile a more detailed field 
Table E1. Comparison of the ElA-23 Sample and Sampling Frame, 1990-1997

\begin{tabular}{|c|c|c|c|c|c|c|c|c|}
\hline \multirow[b]{2}{*}{ Dperator Categor } & \multicolumn{8}{|c|}{ Number of Opeartars } \\
\hline & 1990 & 1991 & 1992 & 1993 & 1994 & 1995 & 1996 & 1997 \\
\hline \multicolumn{9}{|l|}{ Cartainty } \\
\hline Category I. $\ldots \ldots \ldots \ldots$ & 144 & 144 & 157 & 160 & 161 & 161 & 176 & 180 \\
\hline Category II . . . . . . . . . & 468 & 484 & 480 & 500 & 489 & 476 & 486 & 461 \\
\hline Category III . . . . . . . . . . & 2.316 & 2,074 & 1,896 & 1.723 & 1,694 & 1,596 & 3 & 1,194 \\
\hline Sampled $\ldots \ldots \ldots \ldots$ & 2,928 & $2.70 \mathrm{P}$ & 2,533 & 2,383 & 2,337 & 2,233 & 605 & 1,835 \\
\hline Percent Sampled . . . . . . & 100 & 100 & 100 & 100 & 100 & 100 & 100 & 100 \\
\hline \multicolumn{9}{|l|}{ Noncertainty } \\
\hline Sampied ............... & 1,431 & 1,760 & 1,724 & 1,691 & 1,737 & 1,632 & 0 & 1,645 \\
\hline Percent Sampled . . . . . . & 6 & 自 & 8 & B & 8 & 8 & 0 & 8 \\
\hline \multicolumn{9}{|l|}{ Tolal } \\
\hline Active Operators. . . . . . . & 27,556 & $\mathrm{R} 24,846$ & R24,173 & A23,656 & $\mathrm{R} 24,292$ & 22,766 & 23,410 & 22,678 \\
\hline Nö Sampled . . . . . . . & 23,196 & 20,384 & 10,916 & 19.791 & $20,14 B$ & 18,901 & 22,745 & 19,198 \\
\hline Samplad . . . . . . . . . . . . & 4,360 & 4,462 & 4,257 & 4,074 & 4,074 & 3,865 & 685 & 3,480 \\
\hline Percent Sampled . . . . . . . & 18 & 10 & 18 & 17 & 17 & 17 & 3 & 15 \\
\hline
\end{tabular}

ReRevised data

Nore: Acllwe operators in 1997 include 10 cperators added atler Decenter 29, 1997 and nol included in Table E3.

Source: Energy iniomation Admin|stralion, Office of Oil and tias.

Table Ez. Form EIA-23 Survey Response Statistics, 1997

\begin{tabular}{|c|c|c|c|c|c|c|c|c|c|}
\hline \multirow[b]{2}{*}{ Operator Cotingory } & \multirow{2}{*}{$\begin{array}{l}\text { Original } \\
\text { Sample } \\
\text { selected }\end{array}$} & \multirow{2}{*}{$\begin{array}{l}\text { Successoor } \\
\text { Operatore }\end{array}$} & \multirow{2}{*}{$\begin{array}{c}\text { Net } \\
\text { Cotogkiry } \\
\text { Changes }\end{array}$} & \multirow{2}{*}{$\begin{array}{c}\text { Non-c } \\
\text { operalors }\end{array}$} & \multirow{2}{*}{$\begin{array}{l}\text { Adjusted } \\
\text { Sampte }\end{array}$} & \multicolumn{2}{|c|}{$\begin{array}{l}\text { Pesponding } \\
\text { Dparaters }\end{array}$} & \multicolumn{2}{|c|}{$\begin{array}{c}\text { Nonresponding } \\
\text { op arators: }\end{array}$} \\
\hline & & & & & & Number & Perceni & Nomber & PorcenI \\
\hline \multicolumn{10}{|l|}{ Cortesinty } \\
\hline Calegoryl I . . . . & 180 & $\mathbf{3}$ & 0 & -14 & 169 & 169 & 100.0 & 0 & 0.0 \\
\hline Category II . . . . . . & 461 & $\mathbf{5}$ & -11 & $\cdot 37$ & 418 & 418 & 100.0 & 0 & 0.0 \\
\hline Category III . . . . . . & 1,194 & 21 & 11 & .80 & 1,146 & 1,146 & 100.0 & 0 & 0.0 \\
\hline Subrtotel . . . . . . & 1,895 & 29 & 0 & -131 & 1,733 & $1,7,33$ & 100.0 & 0 & Q.D \\
\hline Noncartalnty . . . . . . . & 1,645 & 14 & 0 & 311 & 1,348 & 1,342 & 99.6 & 6 & 0.4 \\
\hline Total $\ldots \ldots \ldots \ldots$ & 3.480 & 43 & 0 & -442 & 3,081 & 3,075 & 99.8 & 6 & 0.2 \\
\hline
\end{tabular}

a Successor operators are those. not Inil ally sempled, that have laken over the production ol a sampled operalor.

Net of recatejontzod operators in the sample (exiltiting nonjperalors\}).

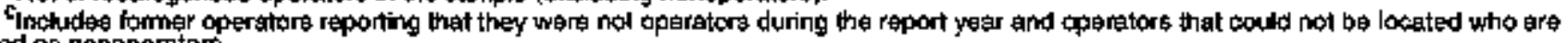
treated as ronoperators.

Ad]usted sample truals oflgina sample plus successor ope ators plus net category chenges minus nonoperators.

Source: Fom ElA-23, "Avinual Sumvey of Domestic, Oit and Gias Reservos" 1997.

level data form. Category III operators file a summary report which is aggregated at a State/subdivision level.

The cover page required of all respondents identifies each operator by name and address (Fjgure I't, Appendix I). The oil and gas producing industry includes a large number of small enterprises. To minimize reporting burden, only a sample of small operators were required to file a summary report of Forrn ELA-23 (Figures I2 and 13, Appendix 1). Report $y$ ear production data were required by State/subdivision areas for crude oil, nahural gas, and lease condensate. Proved reserves data for operators were required only for those properties where estimates existed in the respondent's records.
All Category I and Category II operators were required to file field level data on Schedule A, "Operated Proved Reserves, Production, and Related Data by Field," for each oil and/or gas field in which the respondent operated properties (Figure I4, Appendix 1). All Category I and those Category II operators who had reserve estimates were required to file on a total operated basis for crude oil, nonassociated natural gas, associated-dissolved natural gas, and lease condensate. The following data items were required to be filed: proved reserves at the beginning and the end of the report year, revision increases and revision decreases, extensions, new field discoveries, new reservoirs in old fields, production, indicated additional reserves of crude oil, nonproducing reserves, field discovery year, water depth, and field location information. 
Category II operators who did not have reserves estimates were required to file the field location information and report year production for the four hydrocarbon types from properties where reserves were not estimated. These respondents used Schedule B, "Footnotes," to provide clarification of reported data items when required in the instructions, or electively to provide narrative or detail to explain any data itern filed (Figure [5, Appendix l).

Crude oil and lease condensate volumes were reported rounded to thousands of barrels of 42 U.S. gallons at $60^{\circ}$ Fahrenheit, and natural gas volumes were reported rounded to millions of cubic feet. All natural gas volumes were requested to be reported at $60^{\circ}$ Fahrenheit and a pressure base of 14.73 pounds per square inch absolute. Other minor report preparation standards wete specified to assure that the filed data could be readily proctessed.

\section{Oil and Gas Fleld Coding}

A major effort to create standardized codes for all identified oil or gas fields throughout the United States was implemented during the $19 \$ 2$ survey year. Information from previous lists was reviewed and reconciled with State lists and a consolidated list was created. The publication of the Oil and Gas Field Code Master List 1997, in February of 1998, was the 16th annual report and reflected data collected through October 1997. This publication was mailed to operators to assist in identifying the field code data necessary for the preparation of Form Ela-23. A copy of this publication may be purchased from the National Energy Information Center (see inside cover page).

\section{Form EIA-23 Comparison with Other Data Series}

Estimated crude oil, lease condensate, and natural gas production volumes from Form ELA-23 were compared with official ELA production data supplied by Federal and State oil and natural gas regulatory agencies and published in EIA's monthly and annual reports. Reports published by the Federal and State oil and natural gas regulatory agencies were used to compare specific operator production responses to these agencies with Form ElA-23 responses. When significant differences were found, responses were researched to detect and reconcile possible reporting exrors.
For 1997, Form ElA-23 National estimates of production were 2,312 million barrels for crude oil and lease condensiate or $\mathbf{4 3}$ million barrels (1.8 percent) lower than that reported in the Petroleum Supply Antual 1997 for crude oil and lease condensate. Form EIA-23 National estimates of production for dry natural gas were 19,211 billion cubic feet or 310 billion cubic feet (1.6 percent) higher than the Nafural Gas Annut 1997 for 1997 dry natural gas production.

\section{Form EIA-23 Frame Maintenance}

Operator frame maintenance is a major data quality control effort. Extensive effort is expended to keep the trante as current as possible. The Form ELA-23 frame contains a listing of all coude oil and natural gas well operators in the United States and must be maintained and updated regularly in order to ensure an accurate frame from which to draw the sample for the annual crude oil and natural gas reserves survey. The original frame, ceated in 1977, has been revised annually. In addition, outside sources, such as State publications and computer tapes, and commercial information data bases such as Dwight's Energydata and Petroleum Information, are used to obtain information on operator status and to update addresses for the frame each year.

A maintenance procedure is utilized, using a postcard form with prepaid return postage, to contact possible active crude oil and natural gas well operators presently listed on EIA's master frame, but for whom the listing had not been updated for 2 years. This procedure identifies active operators and nonoperators which improves the frame for future sample selections for the survey. Table E3 provides a summary of changes made to the Form ELA-23 frame of crude oil and natural gas well operators for the 1995 survey mailing. These changes resulted from all frame maintenance activities.

The Form EIA-23 operator franke contained a total of 65,420 entries as of December 29, 1997. Of these, 22,668 were confirmed operators. These are operators who have filed in the past or for whom the EIA has recent production data from an outside source. The remaining operators (including both definite and probable nonoperators) exist as a pool of names and addresses that may be added to the active list if review indicates activity. 
Table E3. Summery of the 1997 Operator Frame Activity, Form ElA-23

\begin{tabular}{|c|c|}
\hline $\begin{array}{r}\text { Total } 1996 \text { Operator Frame } \ldots \ldots \ldots \ldots \ldots \\
\text { Operators. } \ldots \ldots \ldots \ldots \ldots \ldots \ldots \ldots \ldots \\
\text { Nonoperatoss. } \ldots \ldots \ldots \ldots \ldots \ldots \ldots\end{array}$ & $\begin{array}{l}64,17: 2 \\
22,240 \\
41,93: 2\end{array}$ \\
\hline Changes to 1996 Operator Status. . . . . . & \\
\hline $\begin{array}{l}\text { or } \ldots \ldots \\
\text { or } \ldots \ldots\end{array}$ & $\begin{array}{l}177 \\
986\end{array}$ \\
\hline $\begin{array}{l}\text { No Chenges to } 1996 \text { Operator Status. } \\
\text { Operators. . . } \ldots \ldots \ldots \ldots \ldots \ldots \ldots \ldots \\
\text { Nonoperators } \ldots \ldots \ldots \ldots \ldots \ldots \ldots\end{array}$ & $\begin{array}{l}63,009 \\
21,254 \\
41,755\end{array}$ \\
\hline $\begin{array}{l}\text { Additions to } 1996 \text { Operator Frame } \ldots \ldots \\
\text { Operator } \ldots \ldots \ldots \ldots \ldots \ldots \ldots \ldots \ldots \\
\text { Nonoperator. } \ldots \ldots \ldots \ldots \ldots \ldots \ldots\end{array}$ & $\begin{array}{r}1,248 \\
1,237 \\
11\end{array}$ \\
\hline $\begin{array}{l}\text { Total } 1997 \text { Opers } \\
\text { Operators... } \\
\text { Nonoperators }\end{array}$ & $\begin{array}{l}65,420 \\
22,663 \\
42,75 \%\end{array}$ \\
\hline
\end{tabular}

Nole: Includes operator hame activity through December 29, 199:" Soume: Enargy Infomation Adrinilstration, Otlice of Oil and Gas.

\section{Form EIA-64A Survey Design}

The data for this report are also collected on Form ElA-64A "Anntal Report of the Origin of Natural Gas Liquids Production." This section provides information concerning the survey design, response statistics, reporting requiremests, and frame maintenance for Form ELA-64A.

Form ElA-23 for report years 1977 and 1978 required nakural gas well operators to report their nahural gas data on a fully dry basis. It was discovered in the course of those surveys that many operators had little or no knowledge of the extraction of liquids from their produced natural gas streams once custody transfer had taken place. Therefore, these operators reverted to reporting the only natural gas volume data they had in their possession. These volume data were for dryer natural gas than that which had passed through the wellhead, but wetter than fully dry natural gas. With reference to Figure E1, they reported their volumes either at the wellhead or after removal of leass condensate in their lease or field separation facilities.

Some of the larger operators, however, also owned or operated natural gas processing plants. They reported their volumes after removal of both lease condensate and plant liquids, as required by Form EIA-23. The aggregate volumes resulting from the 1977 and 1978 surveys, therefore, were neithcr fully dry (as was intended) nor fully wet. They do appear to have been more dry than wet simply because the operators who reported fully dry volumes also operated properties that contained the bulk of proved natural gas neserves.

The EIA recognized that its estimates of proved reserves of natural gas tiquids (NGL) had to reflect not only those volumes extractable in the future inder current economic and operating conditions at the lease or field (lease condenssate), but also volumes (plant liquids) extractable downstream at existing natural gas processing plants. Form EIA-64, which already canvassed these processing plants, did not request that the plants' production volumes be attributed to source areas. Beginning with the 1979 survey, a new form to collect plant liquids production according to the area or areas where their input natural gas stream had beer produced was mailed to all of the operating plants. The instructions for filing the Form EIA-23 were altered to collect data from natural gas well operators that reflected those volumes of natural gas dried only through the lease or field separation facilities. The reporting basis of these volumes are referred to as "wet after lease separation." The methodology used to estimate NGL reserves by State and State subdivision is provided in Appendix F.

\section{Form EIA-64A Response Statistics}

ELA mailed ELA-64A forms to all known natural gas processing plant operators as of January 10,1998 . In addition, plant operators whose plants were shut down or dismantled during 1997 were requised to complete forms for the portion of 1997 when the plants were in operation.

Natural gas processing plant operators were requested to file a Form ELA-64A for each of their plants. A total of 263 operators of 659 plants were sent forms. This number included 4 new plants, 2 reactivated plants, and 16 successor plants identified after the iritial 1997 survey mailing. A total of 27 plants were reported as nonoperating according to the Form ElA-64A definition. For the eleventh consecutive year the resportse rate was 100 percent.

Form EIA-64A respondents were requested to report natural gas liquids production data by area of origin. Table EA summarizes the responses by plant operators of the volume and origin of natural gas delivered to the 


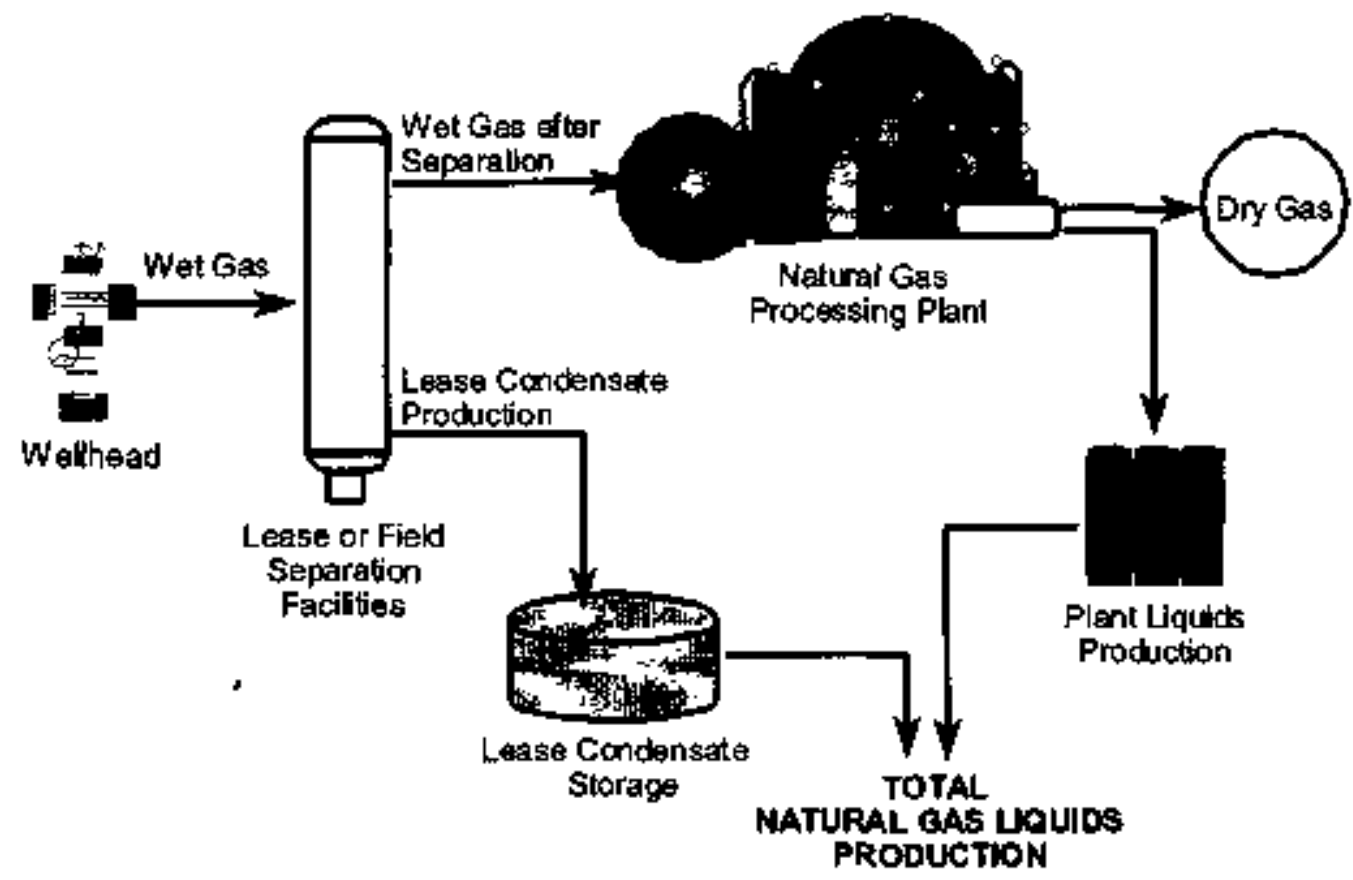

Source: Enengy Information Administration, Office of ONl and Gas.

processing plants and the volume of the natural gas liquids extracted by the plants by State. The majority of the plant operators reported only one area of origin for the natural gas that was processed by a plant. The State or area of origin reported is generally also the plant's location.

\section{Form EIA-64A Reporting Requirements}

Form BIA-64A consisted of the reporting schedule shown in Figure 16, Appendix I. The form identifies the plant, its geographic location, the plant operator's name and address, and the parent company name. The certification was signed by a responsible official of the operating entity. The form pertains to the volume of natural gas received and of natural gas liquids produced at the plant, allocated to each area of origin. Operators also filed the data pertaining to the amount of natural gas shrinkage that resulted from extraction of natural gas liquids at the plant and the amount of fuel used in processing.

Natural gas liquids volumes were reported rounded to thousands of barrels of 42 U.S. gallons at $60^{\circ}$ Fahrenheit, and natural gas volumes were reported rounded to millions of cubic feet. All natural gas volumes were requested to be reported at $60^{\circ}$ Fahrenheit and a pressure base of 14.73 pounds per square inch absolute. Other minor report preparation standards were specified to assure that the filed data could be readily processed.

\section{Form EIA-64A Comparison with Other Data Series}

Form EIA-64A plant liquids production data were compared with data collected on Form EIA-816, "Monthly Natural Gas Liquids Report." Aggregated production from Form ElA-816 represents the net volume of nahural gas processing plant liquid output less input for the report year. These data are published in EIA's Petroleum Supply Annual reports. The Form EIA-64A annual responses reflect all corrections and revisions to EIA's monthly estimates. Differences, when found, were reconciled in both sources. For 1997 . the Form BIA-64A National estimates were 4.1 percent (27 million barrels) higher than the Petroleum Supply Annual $\mathrm{J} 997$ volume for natural gas plant liquids production. 
Table E4. Natural Gas Processed and Liquids Extracted at Natural Gas Processing Plants, 1997

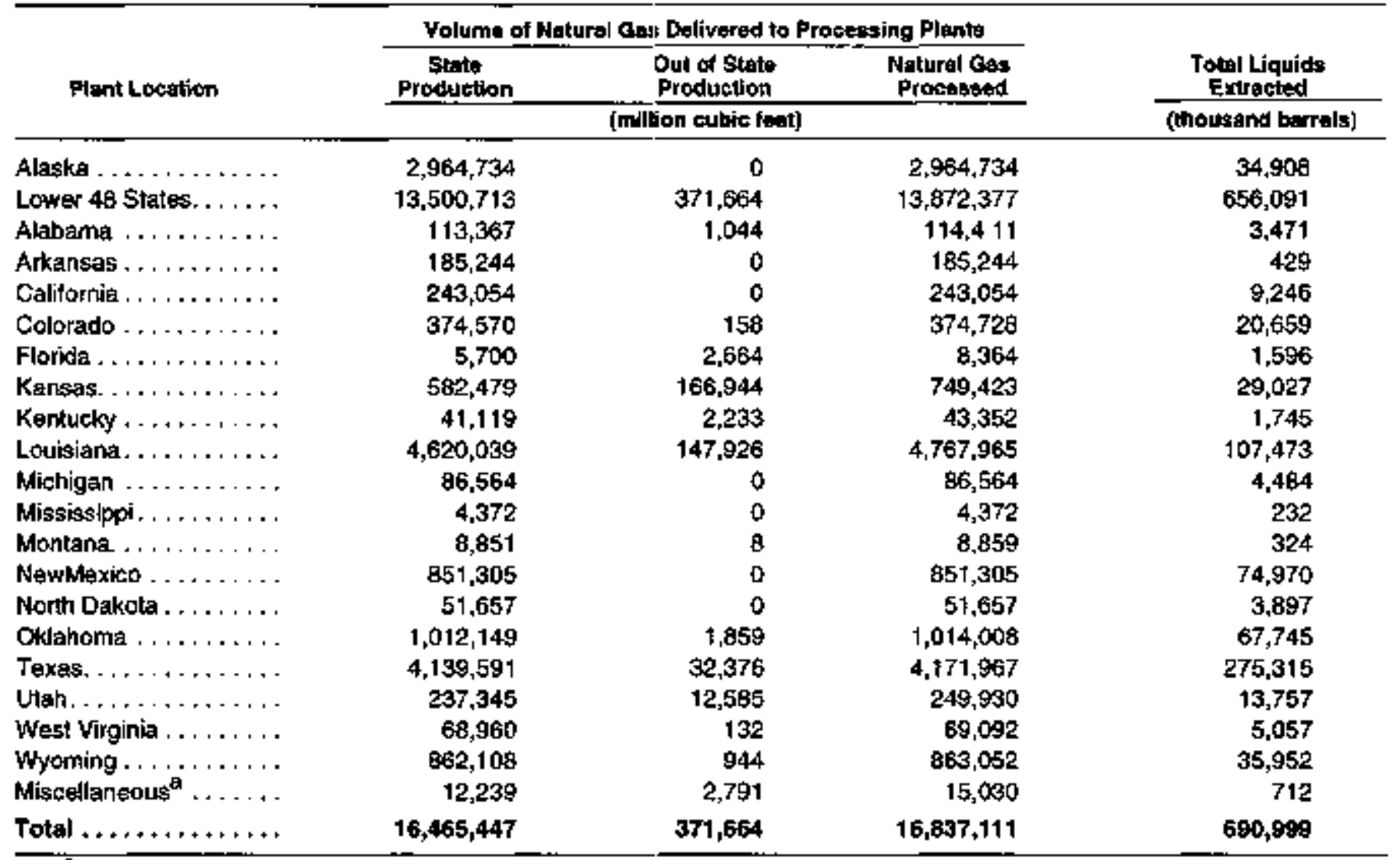

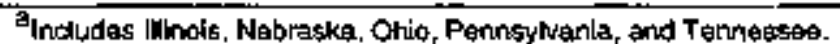

Scurce: Form EkA-64A. 'Annugal Report of the Origh of Mabural Gas Liquids Producticn," 1997.

\section{Form EIA-64A Frame Maintenance}

The Form ElA-64A plant frame contains data on all known active and inactive natural gas processing plants in the United States. The 1997 plant frarne was compared to listings of natural gas processing plants from Form ELA-816, "Monthly Natural Gas Liquids Report"; the LPG Almanac, and the OiI and Gas journa'. A list of possible additions to the plant frame was compiled. Table E5 summarizes the Form ElA-64A plant frame changes made as a result of the comparisons as of December 29, 1997.
Table E5. Form ElA-64A 1997 Plant Frame Actlvity

Frame as of 1996 survey mailing. . . . . . . . . 710 Additions . . . . . . . . . . . . . . . 141

Deletions .................... . .

Frame as of 1997 sunvigy mailing. . . . . . . . . , 654

Note: Indudes operator frame activity through December 29, 1997. Source: Enorgy Intormallon Adrninistration, Oltioe of OA ard Gas. 
Appendix $\mathrm{F}$

Statistical Considerations 



\section{Statistical Considerations}

\section{Survey Methodology}

The Form ElA-23 survey is designed to provide reliable estimates for reserves and production of cude oil, natural gas, and lease condensate for the United States. Operators of crude oil and natural gas wells were selected as the appropriate respondent population because they have access to the most current and detailed information, and therefore, presurnably have better reserve estimates than do other possible classes of respondents, such as working interest or royalty owners.

While large operators are quite well known, they comprise only a small portion of all operators. The small operators are not well known and are difficult to identify because they go into and out of business, alter their corpotate identities, and charige addresses frequtently. As a result, EIA conducts extensive frame maintenance activities each year to identify all current operators of crude oil and natural gas wells in the country-

\section{Sampling Strategy}

EIA publishes data on reserves and production for crude oil, natural gas, and lease condensate by State for most States, and by State subdivision for the States of Callfomia, Louisiana, New Mexico, and Texas. To meet the survey objectives, while minimizing respondent burden, a tandom sampling strategy has been used since 1977. Each operator reporting on the survey is asked to report production for coude oil, natural gas, and lease condersate for each State/subdivision in which he operates. The term State/subdivision refers to an individual subdivision within a State or an individual State that is not subdivided.

The total volume of production varies among the State/subdivisions. To meet the survey objectives while controlling total respondenk burden, EIA selected the following target sampling error for the 1997 survey for each product class.

- 1.0 percent for National estimates.

- 10 percent for each of the 5 States having subdivisions: Alaska, California, Loujsiana,
New Mexico, and Texas. For selected subdivisions within these States, targets of 1.0 percent or 1.5 percent as required to meet the State target.

- 2.5 percent for each State/subdivision having 1 percent or more of estimated U.S. reserves or production in 1994 (lower 48 States) for any product class.

- 4 percent for each State/subdivision having less than 1 percent of estimated U.S. reserves or production in 1994 (lower 48 States) for all 3 product classes.

- 8 percent for States not published separately. The combined production from these States was less than 0.2 percent of the U.S. total in 1994 for crude oil and for natural gas.

The volume of production defining the Certainty stratum, referred to as the cutoff, varies by product or State/subdivision. The cutoff criteria and sampling rates are shown in Table F1. The Certainty stratum, therefore, has three components.

- Category 1 - Large Operators: Operators who produced a total of 1.5 million batrels or more of crude, or 15 bilition cubic feet or more of natural gas, or both in 1994.

- Category II - Miermediate Operators: Operators who produced a total of at least 400,000 barrels of crude oil or 2 bilition cubic feet of natural gas, or both, but less than Category 1 operators in 1994.

- Category Ill - Small Operators: Operators who produced less than the Category tf operators in 1994, but which were selected with certainty. Category III operators were subdivided into operators sampled with certainty (Certainty) and operators that were randomly sampled (Noncertainty).

- Certainty - A small operators who satisfied any of the following critería based upon their production shown in the operator frame:

- Operators with annual crude oil production of 200 thousand barrels or mote, or reserves of 4 million barrels or more; or annual natural gas production of 1 billion cubic feet or more, or reserves of 20 billion cubic feet or more. 


\begin{tabular}{|c|c|c|c|c|c|}
\hline \multirow[b]{2}{*}{ State and Subdivision } & \multicolumn{2}{|c|}{ Production Cutoffs } & \multicolumn{3}{|c|}{$\begin{array}{l}\text { Noncertainty Sample } \\
\text { Number of }\end{array}$} \\
\hline & $\begin{array}{l}\text { Crude Oil } \\
\text { (mbbls) }\end{array}$ & $\underset{\text { (mamcf) }}{\operatorname{Gas}}$ & $\begin{array}{l}\text { Certainty } \\
\text { Operalors }\end{array}$ & $\begin{array}{l}\text { Single State } \\
\text { Operators }\end{array}$ & $\begin{array}{l}\text { Multi-State } \\
\text { Operators }\end{array}$ \\
\hline Alabama Onshore $\ldots \ldots \ldots \ldots \ldots \ldots$ & 107 & 1,000 & 68 & 2 & 1 \\
\hline Alaska. . . . . . . . . . . . . & 0 & 0 & 12 & 0 & 0 \\
\hline 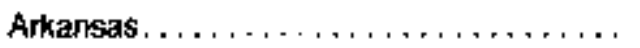 & 21 & 1,000 & 127 & 20 & 3 \\
\hline California Unspecitied. . . . . . . . . . . . & 17 & 88 & 12 & 37 & 0 \\
\hline Calitomia Coastal Reggion Onshore . . . . . . . & 200 & 1,000 & 24 & 1 & 0 \\
\hline Calitorria Los Angelos Basin Onstwore. . . . . & 200 & 25 & 31 & 2 & 0 \\
\hline Calilornia Sen Joaquin Basin Onshora. . . . . & 200 & 1,000 & 46 & 3 & 0 \\
\hline Colorado $\ldots \ldots \ldots \ldots \ldots \ldots \ldots \ldots$ & 200 & 1,000 & 133 & 26 & 7 \\
\hline Florida Onshore . . . . . . . . . . . . & 200 & 1,000 & 6 & 2 & 0 \\
\hline ILineis $\ldots \ldots \ldots \ldots \ldots \ldots \ldots \ldots$ & 200 & 27 & 31 & 37 & 3 \\
\hline Indiana $\ldots \ldots \ldots \ldots \ldots \ldots \ldots \ldots$ & 12 & 1 & 46 & 12 & 2 \\
\hline Kansas $\ldots \ldots \ldots \ldots \ldots \ldots \ldots \ldots$ & 85 & 1,000 & 241 & 174 & 12 \\
\hline Kentucky. . . . . . . . . . . . . . . & 37 & 1,000 & 33 & 21 & 0 \\
\hline Louisiana Unspecified. . . . . . . . . . . . . . & 73 & 183 & 3 & 18 & 0 \\
\hline Louisiana North. , . . , . . . . . . . . . . . & 13 & 630 & 293 & 40 & 7 \\
\hline Lovisiana Soulh Onshore . . . . . . . . . . . . & 70 & 1,000 & 256 & 16 & 2 \\
\hline Michigan . . . . . . $\ldots \ldots \ldots \ldots$ & 200 & 1,000 & 42 & 12 & 2 \\
\hline Missisșippi Onshore . . . . . . . . . . . . . . & 200 & 1,000 & 120 & 11 & 3 \\
\hline Montana $\ldots \ldots \ldots \ldots \ldots \ldots \ldots \ldots$ & 200 & 1,000 & 83 & 11 & 5 \\
\hline Nebraska $\ldots \ldots \ldots \ldots \ldots \ldots$ & 13 & 2 & 54 & 6 & 0 \\
\hline New Mexico Unspecified . . . . . . . . . . . . & 10 & 13 & 12 & 15 & 0 \\
\hline New Mexico Easi $\ldots \ldots \ldots \ldots \ldots \ldots \ldots \ldots$ & 200 & 1,000 & 141 & 3 & 1 \\
\hline New Mexico West . . . . . . . . . . . . . . . & 21 & 1,000 & 54 & 1 & 0 \\
\hline New York $\ldots \ldots \ldots \ldots \ldots \ldots \ldots \ldots$ & 3 & 1,000 & 32 & 48 & 0 \\
\hline North Dakola. . . . . . . . . . . . . . . . . & 200 & 1.000 & 91 & 3 & 8 \\
\hline Ohio $\ldots \ldots \ldots \ldots \ldots \ldots \ldots \ldots \ldots$ & 92 & 1,000 & 44 & 189 & 3 \\
\hline Oklahoma $\ldots \ldots \ldots \ldots \ldots \ldots \ldots \ldots$ & 143 & 1,000 & 341 & 268 & 18 \\
\hline Pennsylvania $\ldots \ldots \ldots \ldots \ldots \ldots \ldots$ & 4 & 1,000 & 80 & 31 & 0 \\
\hline Texas Unspepcified . . . . . . . . . . . . . . . & 7 & 118 & 36 & 257 & 1 \\
\hline Texas-RFC District $1 \ldots \ldots \ldots \ldots$ & 23 & 800 & 196 & 44 & $\mathrm{~g}$ \\
\hline Texas-RRC District 2 Onshore . . . . . . . & 200 & 1,000 & 174 & 8 & 9 \\
\hline Texes-RRC District 3 Onshore . . . . . . . . . & 200 & 1,000 & 252 & 34 & 12 \\
\hline Texas-RRC District 4 Onshore . . . . . . . . . & 91 & 1,000 & 175 & 9 & 8 \\
\hline Texes-RRC Districl $5 \ldots \ldots \ldots \ldots \ldots \ldots$ & 38 & 630 & 98 & 9 & 4 \\
\hline Texas-ARC Disiricl $6 \ldots \ldots \ldots \ldots \ldots$ & 200 & 1,000 & 184 & 21 & 9 \\
\hline Texas-RFC Disiricl $70 \ldots \ldots \ldots \ldots \ldots$ & 34 & 82 & 223 & 72 & 11 \\
\hline Texas-PRC Dislricl $7 \mathrm{C} \ldots \ldots \ldots \ldots \ldots \ldots$ & 200 & 1,000 & 161 & 9 & 4 \\
\hline Texas-RAC District $8 \ldots \ldots \ldots \ldots \ldots$ & 200 & 1,000 & 167 & 5 & 2 \\
\hline Texas-ARC District $8 A \ldots \ldots \ldots \ldots \ldots$ & 200 & 1,000 & 141 & 2 & 2 \\
\hline Texas-ARC District $\mathbf{9} \ldots \ldots \ldots \ldots \ldots$ & 52 & 1,000 & 114 & 12 & 1 \\
\hline Texas-ARC Disirict $10 \ldots \ldots \ldots \ldots \ldots$ & 200 & 1,000 & 123 & 5 & 0 \\
\hline $\operatorname{Utah} \ldots \ldots \ldots \ldots \ldots \ldots \ldots \ldots \ldots$ & 200 & $t, 000$ & 65 & 9 & 1 \\
\hline Virginia $\ldots \ldots \ldots \ldots \ldots \ldots \ldots$ & 200 & 1,000 & t2 & 2 & 0 \\
\hline West Virginia. . . . . . . . . . . . . . & $\mathbf{5}$ & 1,000 & 62 & 35 & 2 \\
\hline Wyoming $\ldots \ldots \ldots \ldots \ldots \ldots \ldots \ldots$ & 200 & 1,000 & 152 & 17 & 6 \\
\hline Offshore Areas . . . . . . . . . . . . . . & 0 & 0 & 286 & 0 & 0 \\
\hline Other Siates ${ }^{\mathrm{a}} \ldots \ldots \ldots \ldots \ldots \ldots \ldots$ & 125 & 49 & 32 & 24 & 0 \\
\hline Total $\ldots \ldots \ldots+\ldots+\ldots+\ldots \ldots+\ldots \ldots+\ldots$ & & & $b_{1,690}$ & 1,563 & $b_{72}$ \\
\hline
\end{tabular}

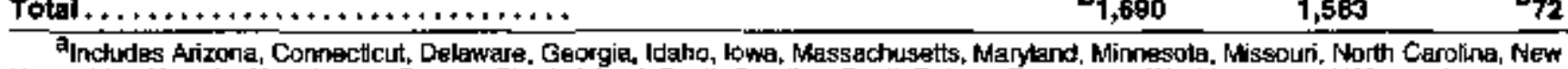
Hampshire, Nevada, New Jersey, Oregon, Rhode Island, South Carolina, South Dakoda, Tennesses, Washington, and Wisconsin.

Eonduplicalive count $\alpha$ operators by States.

Note: Sermpling rate was 8 percent except In Alaska, Florida Onshore, Virginia, and Otkhore areas where sampling rate was 100 percent. - Not gpplicable.

Source: Energy Iniormallon Adtrinistration, Otfice of Oil and sas. 
- All other operators with production or reserves in a State/subdjujsion that exceed selected cutoff levels for that Stake/subdivision.

- The largest operator in each State/subdivision regardless of level of production or reserves.

- Operators with production or reserves of oil or gas for six or more State/stbdivisions.

- Noncertainties - Small opetators not in the certainty stratum were classified in a noncertainty stratum and sampled at a rate of 8 percent.

In each State/subdivision the balance between the number of small certainty operators and the sample size was determined in an iterative procedure designed to minimize the number of total respondents. The iteration for each State/subdivision began with only the Category I and Category II operators in the certainty stratum. The size of the sample of small operators required to meet the target variance was calculated based on the variance of the volumes of those operators. For a number of State/subdivisions with high correlations between frame values across pairs of consecutive years, an adjusted target variance was calculated, that utilized the information about the correlations. This allowed the selection of a smaller sample that still met the target sampling error criteria. At each iteration a small operator, beginning with the largest of the Category III operators, was added to the certainty group and the required sample size was again calculated. The procedure of adding one operator at a time stopped when the proportion of operators to be sampled at random dropped below 8 percent. Independent samples of single location operators (operators who, according to the sampling frame, operate in only one State/subdivision) were selected fom each State/subdivision using systematic randon sampling.

An additional complexity is introduced because some smalt operators selected for the sample in another region or regions, sometimes report production volumes in a region in which Ela has no previous record of production.

State/subdivision volume estimates are calculated as the sum of the certainty strata and all of the estinates for the sampling strata in that region. The sampling variance of the estimated total is the sum of the sampling variances for the sampling strata. There is no sampling ercor associated with the certainty stratum. The square root of the sampling variance is the standard error. It can be used to provide confidence intervals for the State/subdivision totals.

For the States in which subdivision volume estimates are published, the State total is the sum of the individual volume estimates for the subdivisions. The U.S. total is the sum of the State estimates. A sampling variance is calculated for each State subdivision, State, and for the U.S. total.

\section{Total U.S. Reserve Estimates}

Conceptually, the estimates of U.S. reserves and production can be thought of as the sum of the estimates for the individual States. Correspondingly, the estimates for the four States for which estimates are published separately by subdivision (Califorria, Louisiana, New Mexico, and Texas) can be thought of as the sum of the estimates by subdivision. The remaining States are not subdivided and may be considered as a single subdivision.

The estimates of year-end proved reserves and annual production for any State/subdivision is the sum of the volumes in the State/subdivision reported by the certainty stratum operators and an estimate of the total volume in the State/subdivision by the noncertainty stratum operators. Mathematically, this may be stated as the following sum:

$$
\hat{V}_{s}=V_{s}+\hat{V}_{s r}
$$

where

$$
\begin{aligned}
\hat{V}_{s}= & \begin{array}{l}
\text { estimated total volume in the } \\
\text { State/subdivision }
\end{array} \\
V_{s c}= & \begin{array}{l}
\text { total volume in the State/subdjvision } \\
\text { reported by Certainty operators }
\end{array} \\
\hat{V}_{s r}= & \begin{array}{l}
\text { estimated total volume in the } \\
\text { State/subdivision of Noncertainty } \\
\text { operators. }
\end{array}
\end{aligned}
$$

The total volume of Certainty operators in the State/subdivision is simply the sum of individual operator's volumes:

$$
V_{s t}=\sum_{s=1}^{s e c} V_{s c h s}
$$

where

$$
n_{s c}=\underset{\text { number of Certainty operators reporting }}{\text { production in the State/subdivision }}
$$$$
V_{S C M}=
$$ 
volume reported by the $m$-th certainty stratum operator in the State/subdivision.

The estimated total volume of Noncertainty operators in the State/subdivision is the weighted sum of the reports of the noncertainty sample operators:

$$
\hat{V}_{\text {sr }}=\sum_{m=1}^{H} W_{s t r d} V_{s m t r}
$$

where

$$
\begin{aligned}
& n_{S Y} \quad=\text { number of Noncertainty operators } \\
& \text { reporting production in the } \\
& \text { State/subdivision } \\
& V_{5 m I}=\text { volume reported by the } m \text {-th } \\
& \text { Noncertainty sample operator in the } \\
& \text { State/subdivision }
\end{aligned}
$$

In many State/subdivisions, the accuracy of the oil and gas estimates was improved by using a difference estimator for many of the Noncertainty operators. This difference estimator took advantage of the stability of production reports from year-to-year in those State/subdivisions. The difference estimator was only applied to operators who had known production greater than 1 thousand barrels of oil and/or 1 million cubic feet of ratural gas in the previous year. For those State/subdivisions and operators the above formula was modified with $V_{s m m}$ replaced by $V_{s m \text { : }}^{\prime}$ :

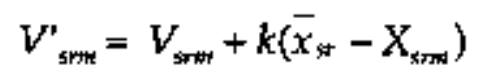

where

$$
\begin{aligned}
k \quad= & 1 \text { when estimating production volumes } \\
k \quad= & \text { regional R/P ratio (Table F6) when } \\
& \text { estimating reserve volumes } \\
\bar{X}_{r}= & \begin{array}{l}
\text { average production volume reported in } \\
\text { the State/subdivision for the preceding } \\
\\
\text { year by all qualifying Noncertainty } \\
\text { operators }
\end{array} \\
X_{\text {Snt }}= & \begin{array}{l}
\text { production volume reported by the m-th } \\
\text { Noncertainty sample operator in the }
\end{array} \\
& \text { State/subdivision for the preceding year. }
\end{aligned}
$$

In selecting the Noncertainty sample, the number of sample operators with production in a given State/subdivision is not controlled to the number expected based on the sampling rate, but is subject to some variation. The weight used is the reciprocal of the achual sampling rate that resulted for the stratum from which the sample operator was selected, rather than the reciprocal of the expected sampling rate. The sample estimate with elther set of weights is an unbjased estimator of the noncertainty strahum total. However, use of the actual sampling rates is expected to lead to smaller sampling errors for the estimates. In making estimates for a State/subdivision, separate weights are applied as appropriate for Noncertainty operators shown in the frame as having had production in only the State/subdivision, for those shown as having had production in that State/subdivision and up to four other States/subdivișions, and for operators with no previous record of production in the State/subdivision. National totals were then obtained by summation of the component totals.

\section{Imputation for Operator Nonresponse}

The response rate for Certainty operators for the 1997 survey was 100 percent. Due to the 100 percent response rate, imputation for the nonresponding operators was not necessary.

\section{Imputation and Estimation for Reserves Data}

In order to estirzate reserve balances for National and State/subdivision levels, a series of imputation and estimation steps at the operator level must be carried out. Year-end reserves for operators who provided production data only were imputed on the basis of their production volumes. Imputation was also applied to the small and intermediate operators as necessary to provide data on each of the reserve balance categories (i.e, tevisions, extensions, or new discoveries). Finally, an imputation was required for the natural gas data of the small operators to estimate their volumes of associated-dissolved and nonassociated natural gas. The firial manipulation of the data accourts for the differences caused by different sample frames from year to year. Each of these imputations generated only a small percentage of the total estimates. The methods used are discussed in the following sections.

The actual data reported on an operated basis by Form EIA-23 respondents for the report year 1997 are summarized in Tables F2, F3, F4, and F5. The differences between these sums and the total estimates shown in Tables 9, 10, 6 and 16 in the main text represent the aggregate result of statistical estimation 
Table F2. Stummary of Total Natura Gas, Wet After Lease Separation, Used in Estimation Process, Form ElA-23 (Million Cubic Feet at 14.73 psie and 60 Degreas Fahrenhẹit)

\begin{tabular}{|c|c|c|c|c|c|}
\hline \multirow[b]{2}{*}{ Level of Reporting } & \multicolumn{5}{|c|}{ Operator Category } \\
\hline & $\mathbf{I}$ & ॥ & Certainty & $\begin{array}{c}\text { Non- } \\
\text { certalnty } \\
\text { ill }\end{array}$ & Total \\
\hline \multicolumn{6}{|l|}{ Fieid Level Reported and Imputed Data } \\
\hline 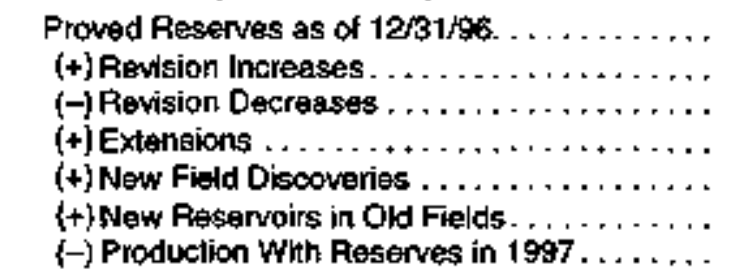 & $\begin{array}{r}146,168,261 \\
18,994,658 \\
14,926,891 \\
9,444,366 \\
2,408,191 \\
2.118,852 \\
16,897,056\end{array}$ & $\begin{array}{r}12,586,503 \\
1,586,968 \\
1,109,696 \\
602,264 \\
213,562 \\
167,369 \\
1,318,388\end{array}$ & $\begin{array}{r}1,573,141 \\
107,048 \\
70,783 \\
201,554 \\
33,000 \\
28,831 \\
155,808\end{array}$ & $\begin{array}{l}- \\
- \\
- \\
- \\
-\end{array}$ & $\begin{array}{r}160,327,905 \\
20,688,674 \\
16,107,370 \\
10,248,184 \\
2,654,753 \\
2,315,052 \\
18,371,252\end{array}$ \\
\hline Proved Reserves Reported as of $12 / 31 / 97 \ldots$. . & $147,490,429$ & $12,729,154$ & $1,716,983$ & - & $161,906,566$ \\
\hline $\begin{array}{l}\text { Prochuction Wihoul Proved Reserves. . . . . . . } \\
\text { Reserves Imputed for Production }\end{array}$ & 0 & 574,585 & 74,224 & - & 648,809 \\
\hline Without Proved Reserves $\ldots \ldots \ldots \ldots \ldots \ldots$ & 0 & $4,376,268$ & 541,666 & - & $4,919,934$ \\
\hline Subtotal Production $\ldots \ldots \ldots \ldots \ldots \ldots$ & $16,897,056$ & $1,892,973$ & 230,032 & - & $19,020,061$ \\
\hline Subtotal Proved Pastarvas $1897 \ldots \ldots \ldots \ldots$ & $147,490,429$ & $17,107,422$ & $2,2,58,648$ & 一 & $166,8,85,500$ \\
\hline \multicolumn{6}{|l|}{ State Level Reporied and Imputed Data } \\
\hline Froduction Wilh Proved Reserves . . . . . . . . & 63 & 47,819 & 141,080 & 12,410 & 201,372 \\
\hline Production Without Proved Resarves . . . . . . . & $Q$ & 32,401 & 224,185 & 40,498 & 297,084 \\
\hline Subtolal Production. . . . . . . . . . . . . . . & 63 & 60,220 & 365,265 & 52,909 & 498,459 \\
\hline Woighted Subtolial Production $\ldots \ldots \ldots \ldots$ & 63 & 60,220 & 365,265 & 676,960 & $1,124,428$ \\
\hline $\begin{array}{l}\text { Proved Feserves Reported. } \ldots \ldots \ldots \ldots \ldots \ldots \\
\text { Reserves Imputed for Production }\end{array}$ & 397 & 376,683 & $1,335,723$ & 93,647 & $1,800,450$ \\
\hline Without Proved Reservas. . . . . . . . . . . & 0 & 227,663 & $1,685,552$ & 311,987 & $2,225,202$ \\
\hline Subtotad Proved Peqserves $\ldots \ldots \ldots \ldots \ldots \ldots$ & 397 & 604,346 & $3,021,205$ & 405,634 & $4,037,582$ \\
\hline Wolghlod subtotal Proved Reservas . . . . . . & 397 & 604,346 & $3,021,206$ & $6,244,768$ & $8,879,718$ \\
\hline Total Production in $1997 \ldots \ldots \ldots \ldots \ldots \ldots$ & $16,897,119$ & $1,973,193$ & 595,297 & 678,980 & $20,144,589$ \\
\hline Tolal Proved Feserves as of $12 / 21 / 97 \ldots \ldots \ldots$ & $147,490,826$ & $17,711,768$ & $5,279,854$ & $5,244,760$ & $175,727,216$ \\
\hline
\end{tabular}

$\rightarrow$ Not applicable.

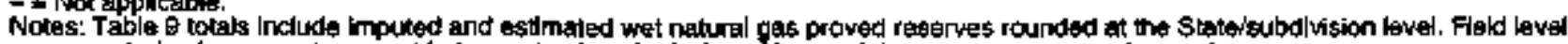
deta are repoited volumes and may not balance due to subrission of incoxmplete reserve component rectords.

Source: Form GIA.23, "Annual survey of Domestic Oil and Gas Regenver," 1997. 
Table F3. Summary of Nonassoclated Natural Gas, Wet After Lsase Separation, Used in Estimation Process, Form ElA-23. (Millicin Cubic Feet at 14.73 psia and 60 Degrees Fahrentheit)

\begin{tabular}{|c|c|c|c|c|c|}
\hline \multirow[b]{2}{*}{ Leval of Reporiting } & \multicolumn{5}{|c|}{ Operator Category } \\
\hline & $\mathbf{I}$ & ॥ & $\begin{array}{c}\text { Certainity } \\
\text { II }\end{array}$ & $\begin{array}{l}\text { Non } \\
\text { cortainty } \\
\text { III }\end{array}$ & Totel \\
\hline \multicolumn{6}{|l|}{ Flald Leval Detail Report } \\
\hline 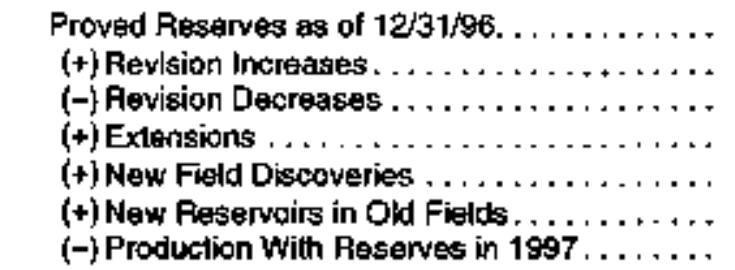 & $\begin{array}{r}119.365,554 \\
14.734,037 \\
12.169,811 \\
8.599,214 \\
1.052,849 \\
1.950,629 \\
14,381,927\end{array}$ & $\begin{array}{r}10,869,057 \\
1,290,719 \\
898,206 \\
553,532 \\
209,565 \\
163,303 \\
1,123,137\end{array}$ & $\begin{array}{r}1,366,076 \\
96,731 \\
61,996 \\
122,047 \\
33,000 \\
21,298 \\
118,786\end{array}$ & $\begin{array}{l}- \\
- \\
- \\
-\end{array}$ & $\begin{array}{r}131,600,687 \\
16,121,487 \\
13,120,013 \\
9,264,793 \\
1,295,414 \\
2,135,223 \\
15,623,650\end{array}$ \\
\hline Proved Resenves Reported as of $12 / 31 / 97 . \ldots$ & $119.140,568$ & $11,075,137$ & $1,458,370$ & - & $131,674,075$ \\
\hline $\begin{array}{l}\text { Production Wikhout Proved Reserves. . . . . . . } \\
\text { Alesenves Implted for Production }\end{array}$ & 0 & 493,355 & 62,391 & - & 555,746 \\
\hline Without Proved Feserves . . . . . . . . . . . . . & $\mathbf{0}$ & $\mathbf{3 , 7 8 1 , 5 1 7}$ & 463,530 & - & $4,245,047$ \\
\hline Sulbtotal Production $\ldots \ldots+\ldots \ldots \ldots \ldots \ldots \ldots$ & $14,381,927$ & $1,616,492$ & 181,177 & - & $16,179,646$ \\
\hline Sublotal Proved Reserves $1997 \ldots \ldots$ & $119,140,568$ & $14,856,654$ & $7,921,900$ & - & $135,919,122$ \\
\hline \multicolumn{6}{|l|}{ Slate Level Reported and Imputed Dese } \\
\hline Production Wilh Proved Reserves . . . . . . . . & - & - & - & - & $\leftarrow$ \\
\hline Production Winhout Proved Reserves . . . . . . . & 一 & 一 & - & 一 & 一 \\
\hline Subtotal Production. . . . . . . . . . . . . . . . . & - & - & - & - & - \\
\hline Weighted Sublotel Production $\ldots \ldots \ldots \ldots$. & - & - & - & - & - \\
\hline $\begin{array}{l}\text { Proved Resenves Reported. . . . . . . . . . . } \\
\text { Resserves Impuled for Production }\end{array}$ & 一 & 一 & 一 & 一 & 一 \\
\hline Writhout Proved Reserves . . . . . . . . . . . & - & - & 一 & - & - \\
\hline Subtotal Proved Reserves $\ldots \ldots \ldots \ldots \ldots \ldots$ & - & - & - & - & - \\
\hline Welghted Subtotel Proved Raservos , . . . . . & 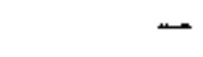 & - & 一 & - & 一 \\
\hline Tokal Proctuction in $1997 \ldots \ldots \ldots \ldots$ & $14,361,927$ & $1,616,492$ & 181,177 & - & $16,179,596$ \\
\hline Total Proved Restrves as of $12 / 31 / 97 \ldots \ldots \ldots$ & $119,140,568$ & $14,856,654$ & $1,921,900$ & - & $135,919,122$ \\
\hline
\end{tabular}

- = Not appllcable.

Notes: Jable 10 totals include imputed and estimated wet natural gas proved reserves rounded at the Stalofsubdivision lovel. Field lovel

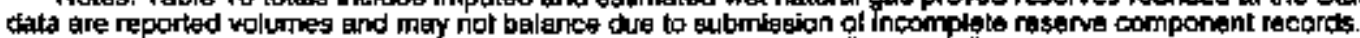

Source: Form EKA-Z3, 'Annual Survey of Domestic Oil and Ges Roservos," 1997. 
Table F4. Summary of Crude Oll Usod in Estimation Process, Form ErA-23 (Thousand Barresls of 42 U.S. Gallons)

\begin{tabular}{|c|c|c|c|c|c|}
\hline \multirow[b]{2}{*}{ Level of Peporting } & \multicolumn{5}{|c|}{ Operator Category } \\
\hline & 1 & ॥ & $\begin{array}{c}\text { Certalnty } \\
\text { nil }\end{array}$ & $\begin{array}{c}\text { Non- } \\
\text { certainty } \\
\text { If }\end{array}$ & Total \\
\hline \multicolumn{6}{|l|}{ Fleld Level Deteril Report } \\
\hline 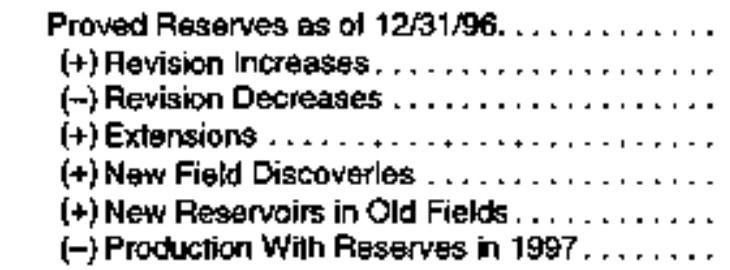 & $\begin{array}{r}19,347,430 \\
1,567,972 \\
799,058 \\
362,706 \\
630,774 \\
110,474 \\
1,759,703\end{array}$ & $\begin{array}{r}1,071,989 \\
168,843 \\
83,613 \\
13,137 \\
2,092 \\
3,453 \\
109,062\end{array}$ & $\begin{array}{r}156,225 \\
10,543 \\
11,890 \\
27,047 \\
0 \\
979 \\
14,030\end{array}$ & $\begin{array}{l}- \\
- \\
- \\
- \\
-\end{array}$ & $\begin{array}{r}20,575,643 \\
1,747,358 \\
894,761 \\
402,890 \\
632,666 \\
714,906 \\
1,882,795\end{array}$ \\
\hline Prowed Reserves Heported as of $12 / 31 / 97 \ldots$. . & $19,460,604$ & $1,066,684$ & 168,874 & - & $20,696,162$ \\
\hline Production Without Proved Reserves. . . . . . . & $\theta$ & 36,491 & 10,243 & - & 46,734 \\
\hline $\begin{array}{l}\text { Peserves Imputed lor Production } \\
\text { Without Proved Peserves. . . . . . . . . . }\end{array}$ & 0 & 248,886 & 72,202 & - & 321,088 \\
\hline Subtotat Production . . . . . . . . . . . . . & $1,759,703$ & 145,553 & 24,273 & - & $1,929,528$ \\
\hline Subtotal Proved Reserves 1997 . . . . . . . & $19,460,604$ & $\mathbf{t , 3 1 5 , 5 7 0}$ & 241,076 & - & $21,017,250$ \\
\hline \multicolumn{6}{|l|}{ Slate Level feporled end Imputed Data } \\
\hline Production With Proved Reserves . . . . . . . . . . & $\mathbf{5}$ & 6,868 & 29,202 & 1,983 & 38,058 \\
\hline Productlon Without Proved Peserves . . . . . . . . & 0 & 4,332 & 46,865 & 7,583 & 58,780 \\
\hline Subiotal Production. . . . . . . . . . . . . . . & $\mathbf{5}$ & 11,200 & 76,034 & 9,566 & 96,805 \\
\hline Weighted Sublotef Production $\ldots \ldots \ldots \ldots \ldots$ & 5 & 11,200 & 76,034 & 123,609 & 210,849 \\
\hline Proved Reserves Reported. . . . . . . . . . . . . & 50 & 51,907 & 236,811 & 16,010 & 304,778 \\
\hline $\begin{array}{l}\text { Resenves Imputed for Production } \\
\text { Without Proved Reserves. } \ldots \ldots \ldots \ldots \ldots \text {. }\end{array}$ & 0 & 30,430 & 321,373 & 53,326 & 405,129 \\
\hline Subiolal Proved Reserves . . . . . . . . . . . & 50 & 62,397 & 558,184 & 69,336 & 709,907 \\
\hline Wrighted Subtotal Proved Reserves . . . . . . & 50 & $6 \mathbf{2}, \mathbf{3} 3 \mathbf{7}$ & 584,529 & $B 33,522$ & $1,540,438$ \\
\hline Totet Production in $1997 \ldots \ldots \ldots \ldots$ & $1,759,70 \mathrm{~B}$ & 166,753 & 100,307 & 123,609 & $2,140,377$ \\
\hline Total Proved fecerves as of 12/31/97, ....... & $19,460,654$ & $1, \$ 97,907$ & 805,605 & 893,522 & $22,557,668$ \\
\hline
\end{tabular}

$-=$ Not applicable.

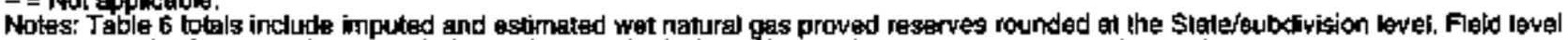
dala zer reported volumes and may hot balance due la submisston of incomplele reserve component records.

Source: Form EIA-23, "Annual Survay of Donestic Oin and Gas Peserves," 1997. 
Table F5. Summary of Lease Condensate Used in Estimation Process, Form ElA-23 (Thousand Barrels of 42 U.S. Gallons)

\begin{tabular}{|c|c|c|c|c|c|}
\hline \multirow[b]{2}{*}{ Level ol Reporting } & \multicolumn{5}{|c|}{ Operalor Calegory } \\
\hline & $\mathbf{1}$ & II & Certainty & $\begin{array}{l}\text { Non- } \\
\text { certainty } \\
\text { III }\end{array}$ & Total \\
\hline \multicolumn{6}{|l|}{ Field Level Detall Report } \\
\hline 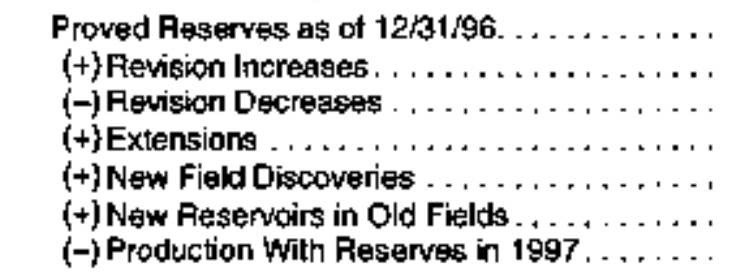 & $\begin{array}{r}1,141,581 \\
195,410 \\
224,356 \\
97,051 \\
53,026 \\
35,245 \\
148,778\end{array}$ & $\begin{array}{r}90,203 \\
19,558 \\
20,609 \\
7,660 \\
6,405 \\
692 \\
12,536\end{array}$ & $\begin{array}{r}8,454 \\
1,075 \\
737 \\
317 \\
94 \\
1,104 \\
1,021\end{array}$ & $\begin{array}{l}- \\
- \\
- \\
-\end{array}$ & $\begin{array}{r}1,240,238 \\
216,043 \\
245,702 \\
105,028 \\
59,525 \\
37,041 \\
162,336\end{array}$ \\
\hline Proved Resenves Reported as of $12 / 31 / 97 \ldots$ & $1,149.187$ & 91,387 & 9,286 & - & $1,249, B 80$ \\
\hline $\begin{array}{l}\text { Production Without Proved Peserves . . . . . . . } \\
\text { Reserves Imputed for Production }\end{array}$ & 0 & 4,342 & 478 & - & 4,820 \\
\hline Without Proved Pesserves . . . . . . . . . . . & $\theta$ & 30,408 & 3,380 & - & \\
\hline Subtotal Production . . . . . . . . . . . . . . & 148,778 & 16,876 & 1,499 & - & 167,155 \\
\hline Subtotal Proved Feserves 1997 . . . . . . . & $1,149,187$ & 121,795 & 12,665 & - & $1,283,649$ \\
\hline \multicolumn{6}{|l|}{ Stale Level Reported and Imputed Data } \\
\hline Production With Proved Reserves . . . . . . . . . & 0 & 293 & 1,293 & 61 & 1,647 \\
\hline Probuction Without Prowed Reserves . . . . . . . . & 0 & 1,003 & 1,075 & 344 & 2,422 \\
\hline Subtotal Production. . . . . . . . . . . . . . & 0 & 1,296 & 2,368 & 405 & 4,069 \\
\hline Welghted Subtotal Production $\ldots \ldots \ldots \ldots$ & 0 & 1,296 & 2,368 & 5,435 & 9,099 \\
\hline Proved Reserves Reported. . . . . . . . . . . . & 1 & 2,747 & 7,758 & 273 & 10,779 \\
\hline Peserves Imputed for Proctuction & & & & & \\
\hline Whthout Proved Aeserves . . . . . . . . . . . & 0 & 7,557 & 7,623 & 2,383 & 17,563 \\
\hline Subtotal Proved Reserves , . . . . . . . . . . . & 1 & 10,304 & 15.381 & 2,656 & 28,342 \\
\hline Wolghted Subtokal Proved Roserves , . . . . & 1 & 10,304 & 15,381 & 35,635 & 61,321 \\
\hline Total Production In $1997 \ldots \ldots \ldots \ldots \ldots$ & 148,778 & 16,174 & 3,867 & 5,435 & 176,254 \\
\hline Total Proved Reearves as of $12 / 31 / 97 \ldots \ldots \ldots$ & $1,149,188$ & 132,099 & 28,047 & 35,635 & $1,344,969$ \\
\hline
\end{tabular}

- =Not applicable.

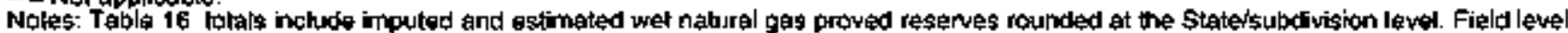
data gire reported volumes and may not balance due to submission of incomplete resarve component records.

Source: Form ElA.23, "Arnuel Sunvy ol Domestlc oll and Gas Ressives," 1997. 
and imputation performed by EIA. The reported data in Table F2 shows that those responding operators accounted for 97.5 percent of the published production for natural gas shown in Table 9 and 93.9 percent of the reserves. Data shown in Table F3 indicate that those responding operators accounted for 94.5 percent of the nonassociated natural gas production and 92.5 percent of the reserves published in Table 10. The reported data shown in Table F4 indicate that those responding operators accounted for 95.1 percent of published crude oil production and 94.1 percent of the reserves shown in Table 6. Additionally, Table F5 indicates that those responding operators accounted for 99.4 percent of the published production and 99.3 percent of the published proved reserves for lease condensate shown in Table 16.

\section{Imputation of Year-End Proved Reserves}

Category I operators were required to submit year-end estimates of proved reserves. Category II and Category III operators were required to provide year-end estimates of proved reserves only if such estimates existed in their records. Some of these respondents provided estimates for all of their operated properties, others provided estimates for only a portion of their properties, and still others provided no estimates for any of their properties. All respondents did, however, provide annual production data. The production reported by Noncertainty sample operators and the corresponding reserves imputed were weighted to estimate the full noncertainty stratum when calculating reserves and production as previously described in the section "Total U.S. Reserves Estimates" in this appendix.
A year-end proved reserves estimate was imputed in each case where an estimate was not provided by the respondent. Reserves were imputed from reported production data for all Noncertainty operators. The reported annual production was multiplied by a reserves-to-production ( $R / P$ ) ratio (Table F6) characteristic of operators of similar size in the region where the properties were located. The regional R/P ratios in this report are averages calculated by dividing the mean of reported reserves by the mean of reported production for selected respondents of similar size who did report estimated reserves. A cutoff level for each region was determined based upon the largest Certainty operator that reported production, but did not provide a reserve estinate. Data from respondents whose production in a region exceeded the regional cutoff level was excluded from the $R / P$ ratio calculation for that region. In addition, operators that had R/P ratios that exceeded 25 to 1 and Category I operators were excluded from the respondents selected to calculate the characteristic regional $\mathrm{R} / \mathrm{P}$ ratjo. All other tespondents who reported both production and reserves were used to calculate the regional $R / P$ ratio characteristic.

The $R / P$ ratio varied significantly from region to region. This variation was presumably in response to variation in geolagic conditions and the degree of development of crude oil and natural gas resources in each area. The average $R / P$ ratio was computed for regional areas similar to the National Petroleum Council regional units (Figure F1). These units generally follow the boundaries of geologic provinces wherein the stage of resource development tends to be somewhat similar. Table $F 6$ lists the $R / P$ ratio

Tabie F6. Statistical Parameters of Reserve Estimation Equation by Peglon for 1997

\begin{tabular}{|c|c|c|c|c|c|c|c|}
\hline \multirow[b]{2}{*}{$\begin{array}{l}\text { Aagion } \\
\text { Number }\end{array}$} & \multirow[b]{2}{*}{ Regton } & \multicolumn{3}{|c|}{ Number ol Narzero RPP Patr: } & \multicolumn{3}{|c|}{ Cheracturieter Multoliers } \\
\hline & & Dil & Aas & $\begin{array}{c}\text { Loente } \\
\text { Cophidenterte }\end{array}$ & 에 & Go. & $\begin{array}{c}\text { Lease } \\
\text { condensate }\end{array}$ \\
\hline 2 & Pacifle Coest States . . . . . . . . & 16 & 24 & 1 & $\mathrm{a}_{6.9}$ & 5.5 & a.1 \\
\hline 3 & Westem Focky Mountalns , . . . . . . & 48 & 51 & 14 & 7.3 & 9.6 & $\mathrm{a}_{7.1}$ \\
\hline 4 & Northern Pocky Mountalns . , , . , & 61 & 63 & $\mathbf{5}$ & 7.8 & 6.1 & $a_{7.1}$ \\
\hline 5 & West Texas and East New Mexico . . . . & 194 & 185 & 43 & 7.5 & 7.0 & $\mathbf{a}_{7.1}$ \\
\hline $6+6 \mathrm{~A}$ & Westem Gulf Basin and Gulf of Mexico. . & 230 & 195 & 160 & 6.5 & 6.7 & 6.9 \\
\hline 7 & Mid-Gontinent . . . . . , . , , & 190 & 141 & 62 & 6.3 & 7.5 & 7.9 \\
\hline $8+9$ & Miçhigan Basin and Eașlem Interkor ... . & 44 & 26 & 7 & 8.7 & 8.7 & $\mathrm{a}_{7.1}$ \\
\hline \multirow[t]{2}{*}{$10+11$} & Appalachians . . . . . . . . . & 22 & 27 & 1 & 7.3 & 11.5 & $\mathbf{\theta}_{7.1}$ \\
\hline & United States . . . . . . . . . . . & 805 & 702 & 299 & 6.9 & 7.5 & 7.1 \\
\hline
\end{tabular}

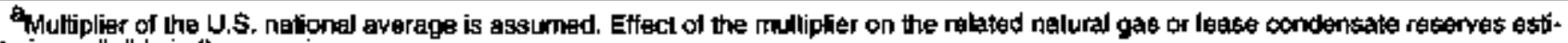
male it negliglble in these regions.

Source: Based on data fileo on Form ElA-23, "Anntag Survey of Domestic Oil and Ges Figserves, 1997. 
calculated for each region that required such imputations and the number of observations on whach it was based

The regronal $R / P$ ratio is determuned prmaruly to provide a factor that can be applied to the production reported by operators without reserve estumates to provide an estunate of the reserves of these operators when aggregated to the regoonal level The average $R / P$ ratio, when multiplied by each individual production in the dustribution of R.P pairs used to calculate it, will exactly reproduce the sum of the reported reserves in the distribution

\section{Imputation of Annual Changes to Proved Reserves by Component of Change}

Category II and Category 111 operators that do not kees reserves data were not asked to provide estmates of begnnung-of-year reserves ot annual changes to proved reserves by component of change, $1 \mathrm{e}$. tevisions, extensions, and discoveries When they did not provide estumates, these volumes were estumated by applyng an algebraic allocation scheme which preserved the relative relationshups between these iterns within each State/subdivision, as reported by Category I and Category II operators, and also preserved an exact annual reserves balance of the following form

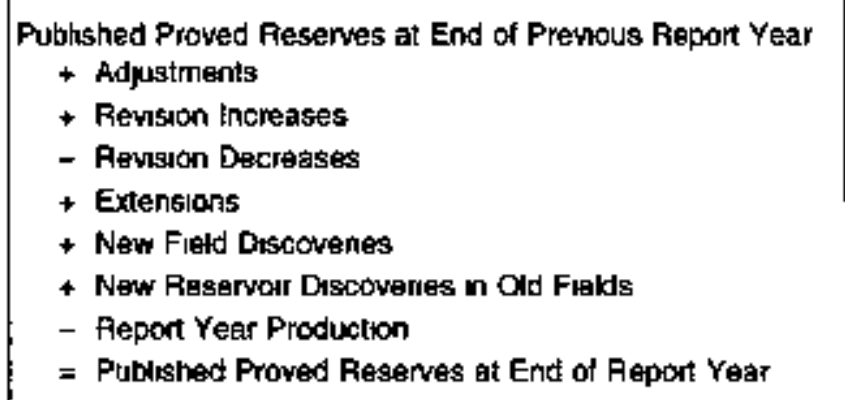

A ratio was calculated as the sum of the arnual production and year-end proved reserves of those

Figure F1. Form ElA-23 Regional Boundarles

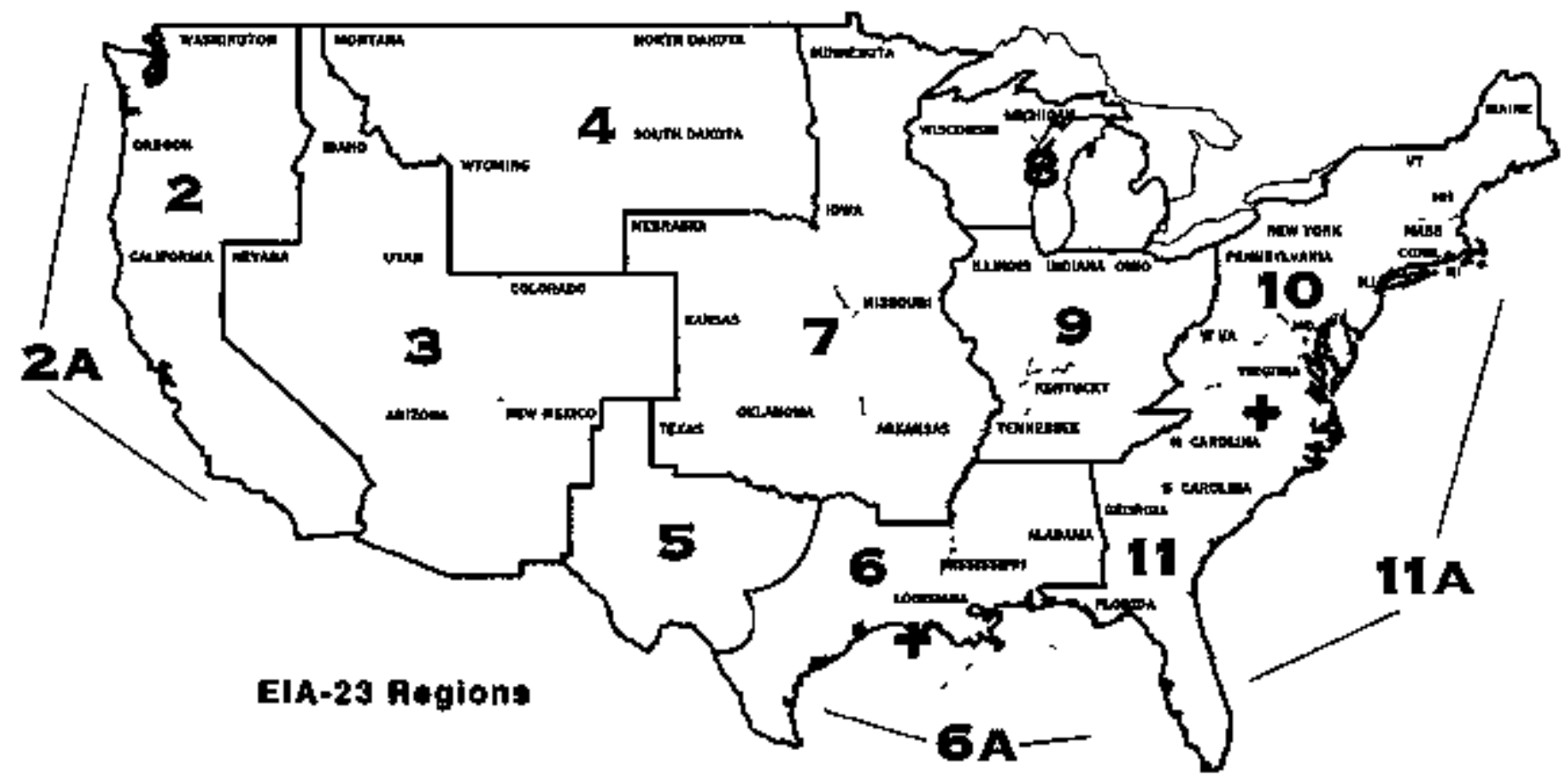

Gouren Energy Intormelion Administration Oifice of Otl end Bac 
respondents who did not provide the reserves balance components, divided by the sum of yearend proved reserves and annual production of those respondents of similar size who did provide these quantities. This ratio was then multiplied by each of the reserves balance components reported by Category $\mathrm{l}$ and some Category II operators, to obtain imputed volumes for the reserves balances of the other Category II operators and Certainty and Noncertainty operators. These were then added to the State/subdivision totals.

\section{Imputation of Natural Gas Type Volumes}

Operators in the State/subdivision certainty and noncertainty strata were not asked to segregate their natural gas volumes by type of natural gas, i.e., nonassociated natural gas (NA) and associated-dissolved natura! gas (AD). The total estimated year-end proved reserves of natural gas and the total annual production of natural gas reported bys or imputed to, operators in the State/subdivision certainty and noncertainty strata were, therefore, subdivided into the NA and AD categories, by State/subdivision, in the same proportion as was reported by Category I and Category $\square$ operators in the same area.

\section{Adjustments}

The instructions for Schedule A of Form EIA-23 specify that, when reporting reserves balance data, the following arithmetic equation must hoid:

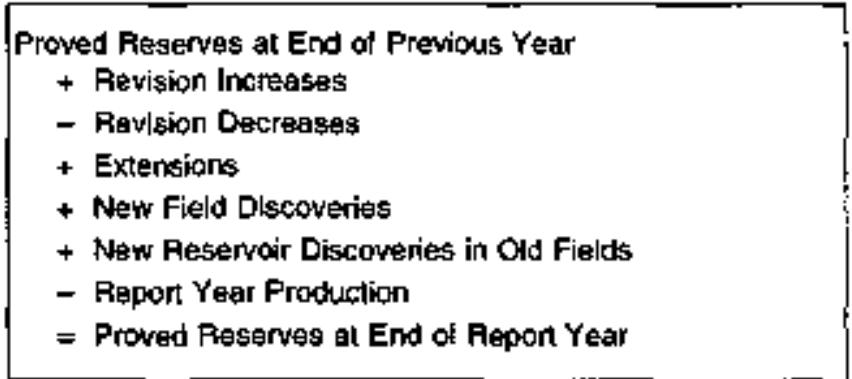

Any remaining difference in the State/subdivision anrual reserves balance between the published previous year-end proved reserves and current yearend proved reserves not accounted for by the imputed reserves changes was included in the adjustments for the area. One of the prinary reasons that adjustments are necessary is that very few of the same Noncertainty operators are sampled each year. Less than 8 percent of the Noncertainty stratum operators sampled in 1995 were sampled again in 1997, and there is no guarantee that in the smaller producing
States/subdivision the same number of small operators will be selected each year, or that the operators selected will be of comparable sizes when paired with operators selected in a ptior year. Thus, some instability of this stratutn from year to year is unavoidable, resulting in minor adjustrnents.

Some of the adjustments are, however, more substantial, and could be required for any one or more of the following reasons:

- The frame coverage may or may not have improved between survey years, such that more or fewer Certainty operators were included in 1997 than in 1996.

- One or more operators may have reported data incorrectly on Schedule $A$ in 1996 or 1997, but not both, and the error was not detected by edit processing.

- Operation of properties was transferred during 1997 from operators not in the frame or Noncertainty operators not selected for the sample to Certainty operators or Noncertainty operators selected for the sanple.

- Operations of properties was transferred during 1997 to an operator with a different evaluation of the proved reserves associated with the properties than that of the 1996 operator.

- Respondent changed classification of natural gas from NA to $A D$ or vice versa.

- The trend in reserve changes imputed for the small operators, that was based on the trend reported by the large operatom, did not reflect the actual trend for the small opetators.

- Noncertainty operators, who have grown substantially in size since they were added to the frame, occasionally cause a larger standard error than expected.

- The Noncertainty sample for either year in a state may have been an unusual one.

The causes of adjustments are known for some but not all areas. The only problems whose effects cannot be expected to balance over a period of several years are those associated with an inadequate frame or those associated with any actual trend in reserve changes for small operators not being the same as those for large operators. ElA continues to attempt to improve sources of operator data to resolve problems in frame completeness. 


\section{Sampling Reliability of the Estimates}

The sample of Noncertainty operators selected is only one of the large number of possible samples that coult have been selected and each would have resulted in different estintates. The standard error or sampling error of the estimates provides a measure of this variability. When probability sampling methods are used, as in the EIA-23 survey, the sampling error of estimates can also be estimated from the survey data.

The estimated sampling error can be used to compute a confidence interval around the survey estimate, with a prescribed degree of confidence that the interval covers the value that would have been obtained if all aperators in the frame had been surveyed. If the estimated volume is denoted by $\hat{v}_{\text {, }}$ and its sampling error by S.E. ( $\hat{V}_{s}$ ), the confidence interval can be expressed as:

$$
\hat{V}_{s}+-k S . E \cdot\left(\hat{V}_{s}\right)
$$

where $\mathbf{k}$ is a multiple selected to provide the desired level of confidence. For this survey, $k$ was taken equzl to 2 . Then there is approximately 95 percent confiderice that the interval:

$$
\hat{V}_{s}+-2 S \cdot E_{1}\left(\hat{V}_{*}\right)
$$

includes the universe value, for both the estimates of reserves and production volumes. Correspondingly, for approximately 95 percent of the estimates in this report, the difference between the published estimate and the value that would be found from a complete survey of all operators is expected to be less than twice the sampling error of the estimate. Tables F7, F8, F9, and F10 provide estitrates for $25 . \mathrm{E} .(\hat{V})$ by product. These estimates are directly applicable for constructing approximate 95 percent confidence intervals. For example, the 95 percent confidence interval for dry natural gas proved reserves is 165,146 \pm 575 billion cubic feet. The sampling error of $\hat{V}_{\mathrm{t}}$ is equal to the sampling error of the noncertainty estimate $\hat{V}_{\mathrm{a}}$, because the certainty total is not subject to sampling error. The estimated sampling error of a noncertainty? estimate is the square root of its estimated sampling variance.

The noncertainty estimate for a given State/subdivision had two separately weighted components based on reports of:

- Type 1 Operators shown in the frame as having had crude sil or natural gas production in the State/subdivision.
- Type 2 Operators shown in the frame as having had nocrude oil or natural gas production in the State/subdivision.

Correspondingly, the sampling variance had two components associated with the estimated production from each component:

$$
\operatorname{Var}\left(\hat{V}_{\mathrm{sr}}\right)=\operatorname{Var}\left(\hat{V}_{\mathrm{sr} 1}\right)+\operatorname{Var}\left(\hat{V}_{s 2}\right)
$$

The $\operatorname{Vor}\left(V_{s r}\right)$ was tirnated as the sum of the estimated variances of the two component estimates. The variance for any component, say component $j$. was estimated from the formula:

$$
\operatorname{Var}\left(\hat{V}_{s i j}\right)=n_{s r i}\left(\frac{W_{s r j}-1}{W_{s r j}}\right) S^{2}
$$

In general, $\hat{V}_{s j}$ denotes the production estimate from component $j$ for each of the two types of operator, and $V a r\left(V_{\text {sir }}\right.$ denotes its variance where

$$
\left.n_{s r j}=\underset{c o m b e r}{\text { nomponent }}\right\}
$$$$
w_{s r j}=\text { weight for operator reports in component } j
$$$$
S^{2}{ }_{s y j}=\text { variance between operator reports in }
$$
component $j$.

If the subscripts $s r$ are dropped, $s^{2}$ tri tan be expressed as:

$$
S^{2} j=\frac{\sum_{i}^{n_{1}} V^{\prime 2} j i-\left(\sum_{i}^{n_{i}} V_{j l}^{\prime}\right)^{2} / n_{j}}{n_{j}-1}
$$

where

$V_{j r}=$ weighted production or reserves volume for the $i$-th sample operator in the component $j$.

The variance of the estimated total volume for a State having subdivisions is the sum of corresponding Type 1 and Type 2 corrponents where the classification of operators by type is with regard to the State as a whole; e.g., Type 2 operators at the State level are those that were not shown in the sample frame as having production anywhere in the State. 
Table F7. Factors for Confidence Intervals (2S.E.) for Dry Natural Gas Proved Reserves and Production, 1997 (Billion Cubic Feet at 14.73 psia and 60 Degrees Fahrenheit)

\begin{tabular}{|c|c|c|c|c|c|}
\hline Stake and Subthisiont & $\begin{array}{c}1997 \\
\text { Peservad }\end{array}$ & $\begin{array}{c}1997 \\
\text { Production }\end{array}$ & State and Subdivision & $\begin{array}{c}1997 \\
\text { Aestryes }\end{array}$ & $\begin{array}{c}1997 \\
\text { Production }\end{array}$ \\
\hline Uriled Steles . . . . . . . . . . . . . . . & 791 & 101 & Oklahoma $\ldots \ldots \ldots \ldots \ldots \ldots$ & $\$ 11$ & 40 \\
\hline Alabama $\ldots \ldots \ldots \ldots \ldots \ldots \ldots$ & 19 & 3 & Pennsylvania . . . . . . . . . . . . . & 174 & \pm 5 \\
\hline Alaskn $\ldots \ldots \ldots \ldots \ldots \ldots \ldots \ldots$ & 0 & 0 & 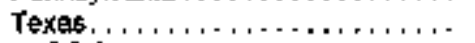 & 435 & 65 \\
\hline Arkansas $\ldots \ldots \ldots \ldots \ldots \ldots$ & 32 & 4 & RAC District $1 \ldots \ldots \ldots \ldots \ldots$ & 17 & 3 \\
\hline Califomia $\ldots \ldots \ldots \ldots \ldots \ldots$ & 47 & 11 & RAC District 2 Onshore . . . . . . . & 123 & 18 \\
\hline Coesial Reglon Onshore. . . . . . . & 0 & 0 & RRC District I Onshore . . . . . . & 86 & 13 \\
\hline $\begin{array}{l}\text { Los Anpeles Basin Onshore . . . . } \\
\text { San Jogequir Basin Onshore. . . }\end{array}$ & 95 & & RRC Dislicet 4 Onshotr . . . . . . & $17 \theta$ & 26 \\
\hline $\begin{array}{l}\text { San Joaquir Basin Onshore . . . } \\
\text { Stara Offshore. . . . . . . . . . }\end{array}$ & $\begin{array}{r}95 \\
0\end{array}$ & $\begin{array}{r}20 \\
0\end{array}$ & RRC District $5, \ldots \ldots \ldots \ldots \ldots$ & 8 & 3 \\
\hline $\begin{array}{l}\text { Statia Offshore. . . } \ldots \ldots \ldots \ldots \ldots \\
\text { Colorado } \ldots \ldots \ldots \ldots \ldots \ldots\end{array}$ & 75 & B & FRC Disinict $6 \ldots \ldots \ldots \ldots \ldots$ & 137 & 20 \\
\hline & 0 & 0 & FRC Disurict $7 B \ldots \ldots \ldots \ldots$ & 28 & 4 \\
\hline Kansas. . . . . . . . . . . . . . . & 138 & 18 & RAC District $7 C . \ldots \ldots \ldots \ldots \ldots$ & 61 & 9 \\
\hline Kentucky , . . . . . . . . . . . . & 58 & 7 & ARC Disinct \& $\ldots \ldots \ldots \ldots \ldots$ & 13 & 2 \\
\hline Louisigna . . . . . . . . . . . . . . . & 119 & 18 & FRC District 8A $\ldots \ldots \ldots \ldots \ldots$ & 0 & 0 \\
\hline North. ..................... & 43 & 6 & RRC District $9 \ldots \ldots \ldots \ldots$ & 12 & 2 \\
\hline South Onshore $\ldots \ldots \ldots \ldots \ldots$ & 103 & 15 & RRC Districl $10 \ldots \ldots \ldots \ldots$ & 12 & 2 \\
\hline Staik Offshors. . . . . . . . . . & 0 & 0 & State Ofishore. . . . . . . . . . & 0 & 0 \\
\hline Michigan $\ldots \ldots \ldots \ldots \ldots \ldots$ & 96 & 11 & Utah $\ldots \ldots \ldots \ldots \ldots \ldots \ldots \ldots$ & 11 & 1 \\
\hline Mississippi $\ldots \ldots \ldots \ldots \ldots \ldots \ldots$ & 26 & $\mathbf{5}$ & 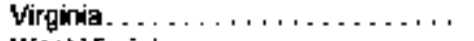 & 0 & 0 \\
\hline 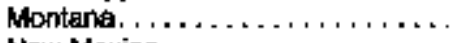 & 12 & 2 & West Virginla $\ldots \ldots \ldots \ldots \ldots \ldots$ & 249 & 11 \\
\hline Naw Hexico. . . . . . . . . . . . . . . & 125 & 14 & Wyoming $\ldots \ldots \ldots \ldots \ldots$ & 297 & 36 \\
\hline East. $\ldots \ldots \ldots \ldots \ldots \ldots \ldots$ & 12 & 3 & Federal Otlshore ${ }^{\mathrm{a}} \ldots \ldots \ldots \ldots \ldots$ & 0 & 0 \\
\hline West $\ldots \ldots \ldots \ldots \ldots \ldots \ldots$ & 0 & 0 & Pacific (Cedifor ria) . . . . . . . . . & 0 & 0 \\
\hline 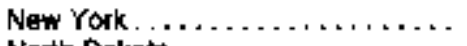 & 72 & 7 & Gulf of Mexico (Lowisiana) ${ }^{\mathrm{B}}$... & 0 & 0 \\
\hline North Dakota $\ldots \ldots \ldots \ldots \ldots \ldots$ & 10 & 1 & Gulf of Maxich (Texas\} . . . . . . . & 0 & 0 \\
\hline 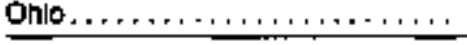 & 141 & 15 & Miscollantous ${ }^{b}, \ldots \ldots \ldots \ldots, \ldots$ & 10 & 1 \\
\hline
\end{tabular}

ancludes Federal offshore Alabame

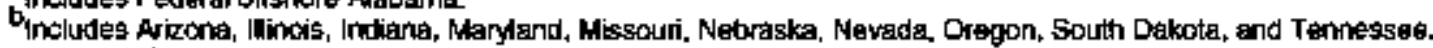

Nodęs: Canlidence intervels are aesoctated wilh Tables 8 reserves and production data. Factors for confidence intervals for each State subdivision, State, and the United States are Independestly estmated and do nol add.

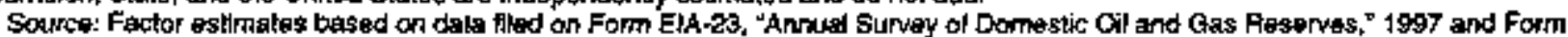

ElA.64h, "Annual Report ol the Orlgin of Natural Gas Liquide Production," 1997.

Table F8. Factors for Confldence Intervals (2S.E.) for Natural Gas Proved Roservos and Productlon, Wet After Lease Separation, 1997 (Bitllon Cublc Feet at 14.73 psia and 60 Degrees Fahrenherit)

\begin{tabular}{|c|c|c|c|c|c|}
\hline State and Subdivlation & $\begin{array}{c}1997 \\
\text { Rencerves }\end{array}$ & $\begin{array}{c}1997 \\
\text { Production } \\
\end{array}$ & Stute and Subdivlsion & $\begin{array}{c}1997 \\
\text { Roservote }\end{array}$ & $\begin{array}{c}1997 \\
\text { Productiten }\end{array}$ \\
\hline 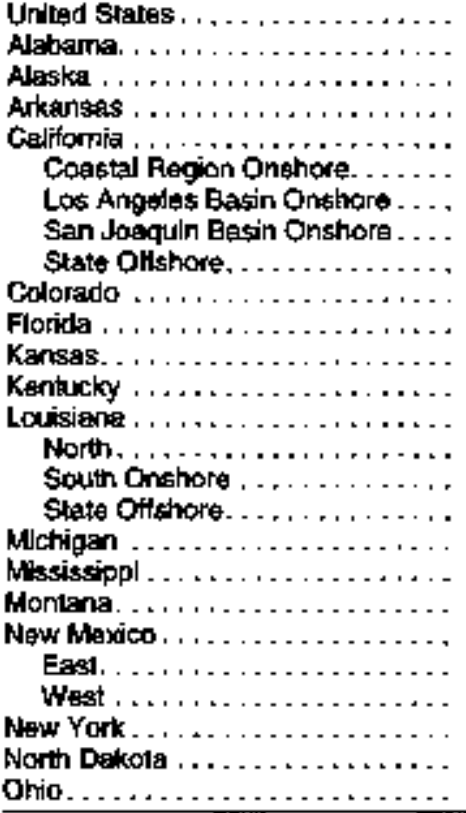 & $\begin{array}{r}836 \\
20 \\
0 \\
32 \\
50 \\
0 \\
3 \\
100 \\
0 \\
84 \\
0 \\
145 \\
60 \\
124 \\
44 \\
108 \\
0 \\
98 \\
26 \\
12 \\
136 \\
14 \\
0 \\
72 \\
12 \\
141\end{array}$ & $\begin{array}{r}107 \\
3 \\
0 \\
4 \\
12 \\
0 \\
0 \\
21 \\
0 \\
9 \\
0 \\
19 \\
8 \\
19 \\
7 \\
16 \\
0 \\
11 \\
5 \\
2 \\
15 \\
3 \\
0 \\
7 \\
1 \\
15\end{array}$ & 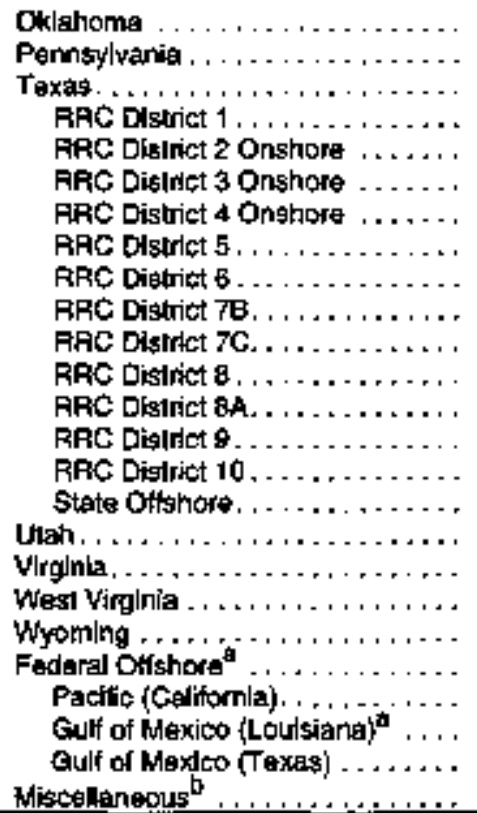 & $\begin{array}{r}332 \\
175 \\
465 \\
18 \\
131 \\
91 \\
187 \\
9 \\
144 \\
32 \\
69 \\
15 \\
0 \\
15 \\
13 \\
0 \\
13 \\
0 \\
257 \\
303 \\
0 \\
0 \\
0 \\
0 \\
10\end{array}$ & $\begin{array}{r}42 \\
15 \\
70 \\
3 \\
19 \\
14 \\
27 \\
3 \\
21 \\
5 \\
10 \\
2 \\
0 \\
2 \\
2 \\
0 \\
1 \\
0 \\
12 \\
97 \\
0 \\
0 \\
0 \\
0 \\
1\end{array}$ \\
\hline
\end{tabular}

akcludes Federal offhore Alabama.

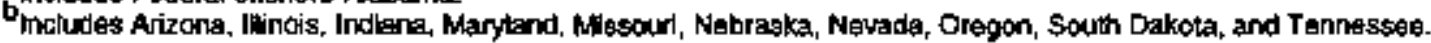

Notes: Confidence intervals are assoctated with Toltle 9 reservas and production deta. Factors for confidence Intelvals for sach \$tale

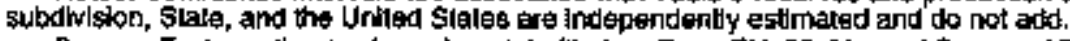

Source: Factor epelimates based on dala filed on Form ElA-23, "Annual Survey of Domestic Oil and Cas feserves," 1997. 
Table F9. Factors for Confidence Intervals (2S.E.) for Crude Oil Proved Reserves and Productlon, 1997 (Million Barrels ol 42 U.S. Gallons)

\begin{tabular}{|c|c|c|c|c|c|}
\hline State and \$ubdivision & $\begin{array}{c}1997 \\
\text { Reserves }\end{array}$ & $\begin{array}{c}1997 \\
\text { Production: }\end{array}$ & State and Subdivision & $\begin{array}{c}\text { t\$s? } \\
\text { Reservos }\end{array}$ & $\begin{array}{c}1997 \\
\text { Produetionn }\end{array}$ \\
\hline 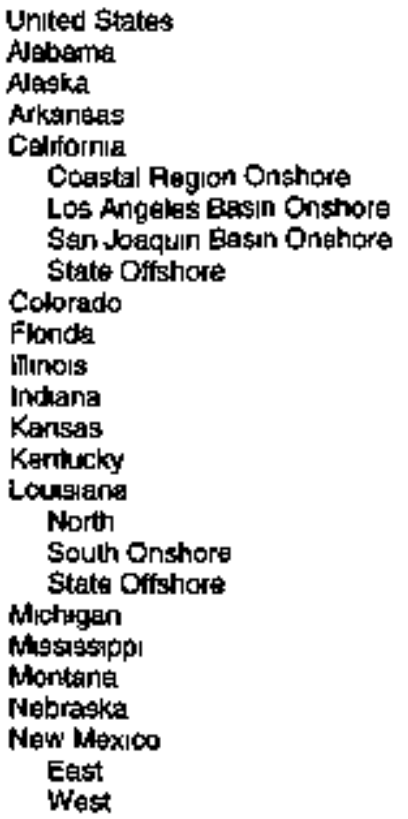 & $\begin{array}{r}108 \\
2 \\
0 \\
3 \\
27 \\
0 \\
2 \\
24 \\
0 \\
11 \\
0 \\
12 \\
2 \\
58 \\
33 \\
17 \\
2 \\
12 \\
0 \\
9 \\
6 \\
10 \\
6 \\
10 \\
10 \\
0\end{array}$ & $\begin{array}{r}13 \\
0 \\
0 \\
1 \\
3 \\
0 \\
0 \\
0 \\
0 \\
1 \\
0 \\
2 \\
0 \\
3 \\
0 \\
2 \\
0 \\
2 \\
0 \\
1 \\
1 \\
1 \\
0 \\
1 \\
1 \\
0\end{array}$ & 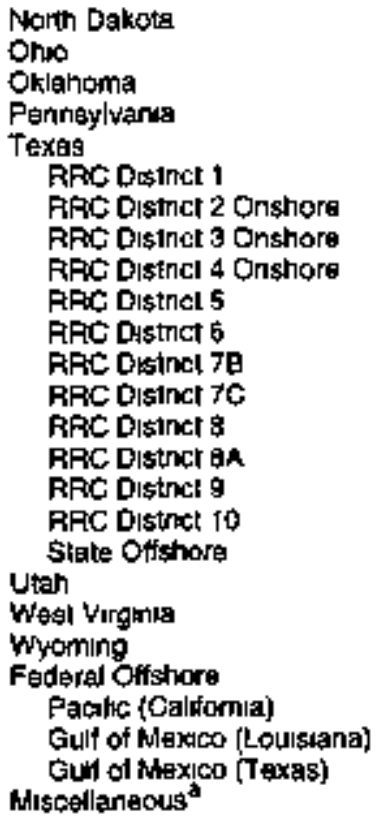 & $\begin{array}{r}10 \\
11 \\
38 \\
1 \\
70 \\
5 \\
5 \\
14 \\
5 \\
3 \\
12 \\
10 \\
8 \\
5 \\
5 \\
4 \\
3 \\
0 \\
8 \\
1 \\
29 \\
0 \\
0 \\
0 \\
0 \\
2\end{array}$ & $\begin{array}{l}1 \\
1 \\
5 \\
0 \\
9 \\
1 \\
1 \\
2 \\
1 \\
0 \\
2 \\
1 \\
1 \\
1 \\
1 \\
0 \\
0 \\
0 \\
1 \\
0 \\
0 \\
0\end{array}$ \\
\hline
\end{tabular}

anchides Anzona, Missoun, Neyada, Now York, South Dakole, Tennesssee, and Virguma

Noles Contudence intervals are associated writh Table 6 reserves and produchon data Factors for contidence inlervals for each State subdivision. State, and the United Silales are independently eotwmated and do not add

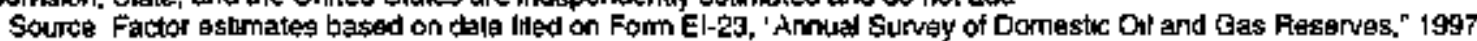

Table F10. Factors for Confidence Intervals (2S.E.) for Lease Condensate Proved Reserves and Production, 1997 (Whillюn Bamels of 42 L.S. Galtons)

\begin{tabular}{|c|c|c|c|c|c|}
\hline Strte and Subdirision & $\begin{array}{c}1997 \\
\text { Aoservas }\end{array}$ & $\begin{array}{c}1997 \\
\text { Productioit }\end{array}$ & Stat and Suhdrutisen & $\begin{array}{c}1997 \\
\text { Reserves }\end{array}$ & $\begin{array}{c}1997 \\
\text { Production }\end{array}$ \\
\hline 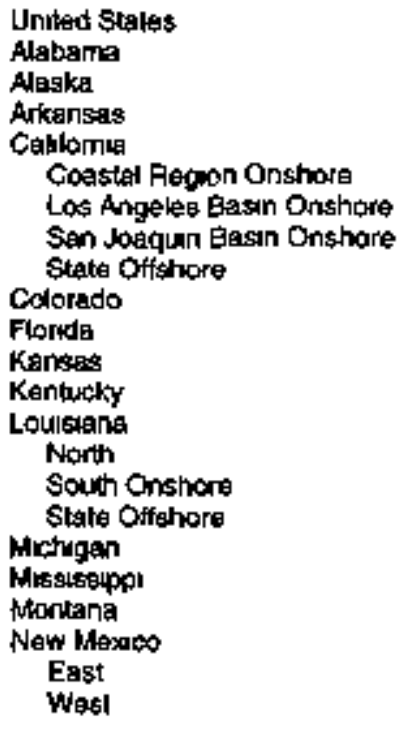 & $\begin{array}{r}36 \\
0 \\
0 \\
0 \\
1 \\
0 \\
0 \\
1 \\
0 \\
0 \\
0 \\
0 \\
0 \\
19 \\
17 \\
0 \\
0 \\
0 \\
0 \\
0 \\
0 \\
0 \\
0\end{array}$ & $\begin{array}{l}5 \\
0 \\
0 \\
0 \\
0 \\
0 \\
0 \\
0 \\
0 \\
0 \\
0 \\
0 \\
0 \\
3 \\
3 \\
0 \\
0 \\
0 \\
0 \\
0 \\
0 \\
0 \\
0\end{array}$ & 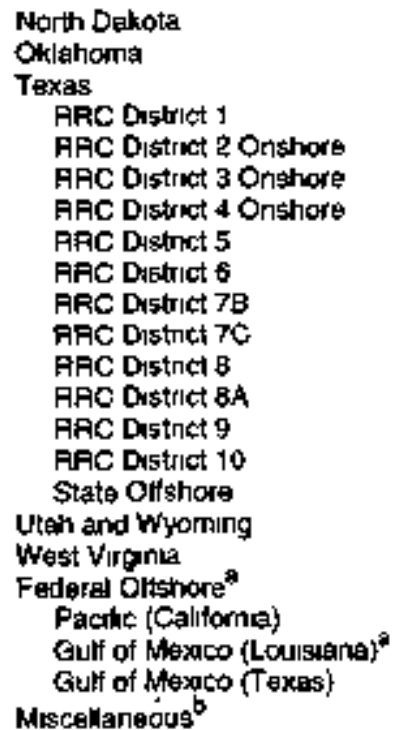 & $\begin{array}{r}0 \\
0 \\
32 \\
0 \\
0 \\
32 \\
2 \\
0 \\
2 \\
0 \\
0 \\
0 \\
0 \\
0 \\
0 \\
0 \\
1 \\
0 \\
0 \\
0 \\
0 \\
0 \\
0\end{array}$ & $\begin{array}{l}0 \\
0 \\
5 \\
0 \\
0 \\
5 \\
0 \\
0 \\
0 \\
0 \\
0 \\
0 \\
0 \\
0 \\
0 \\
0 \\
0 \\
0 \\
0 \\
0 \\
0 \\
0\end{array}$ \\
\hline
\end{tabular}

ancludes Federal offshore Alabama

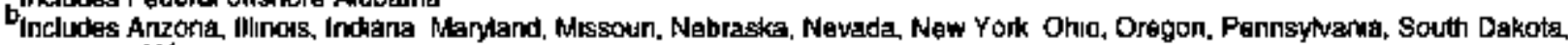
Ternossee, and Vingina

Notes Confidence untervals are assochated wilh Table 16 reserveg and producton dala Factors for conindence intervals for each Stale subdrusson, State, and the Unted States are indeperwdentty estimaled and do mol add

Source Factor egtumales based on data flled on Form ElA-23, Annual Survey of Domesilc Oll and Gag Regerves, '1997 
Since there are no operators in the frame who would be classified as Type 2 at the U.S. level, there would be no Type 2 components at the U.S. level. Therefore, at the U.S. level, there was only one sample variance companent calculated for Type 1 operators.

\section{Nonsampling Errors}

Several sources of possible error, apart from sampling error, are associated with the Form ElA-23 survey. These include bias due to nonresponse of operators in the sample, proved reserve estimation errors, and reporting errors on the part of the respondents to the survey. On the part of ELA, possible errors include inadequate frame coverage, data processing error, and errors associated with statistical estimates. Each of these sources is discussed below. An estimate of the bias from nonuesponse is presented in the section on adjustment for operator nonresponse.

\section{Assessing the Accuracy of the Reserve Data}

The EEA maintains an evaluation program to assess the accuracy and quality of proved reserve estimates gathered on Form ElA-23. Field teams consisting of petroleum engineers from ELA's Dallas Field Otfice conduct technical reviews of reserve estimates and independently estimate the proved reserves of a statistically selected sample of operator properties. The results of these reviews are used to evaluate the accuracy of reported reserve estimates. Operators are apprised of the team's findings to assist them in completing future filings. The magnitude of errors due to differences between reserve volumes submitted by operators on the Form ELA-23 and those estimated by ELA petroleum engineers on their field trips were generally within accepted professional engineering standards.

\section{Respondent Estimation Errors}

The principal data elements of the Form ELA-23 survey consist of respondent estimates of proved reserves of crude oil, natural gas, and lease condensate. Unavoidably, the respondents are bound to make some estimation errors, i.e., until a particular reservoir has been fully produced to its economic limit and abandoned, its reserves are not subject to direct measurement but must be inferred from linited, imperfect, or indirect evidence. A more comptete discussion of the several techriques of estimating proved reserves, and the many problems inherent in the task, appears in Appendix G.

\section{Reporting Errors and Data Processing Errors}

Reporting errors on the part of respondents are of definite concern in a survey of the magnitude and complexity of the Form ELA-23 progran. Several steps were taken by EIA to minimize and detect such problems. The survey instrament itself was carefully developed, and included a detailed set of instructions for filing data, subject to a common set of defiritions similar to those already used by the industry. Editing software is continually developed to detect different kinds of probable reporting errors and flag them for resolution by analysts, either through confirmation of the data by the respondent or through subarission of amendments to the filed data. Data processing errors, consisting primarily of random keypunch errors, are detected by the same software.

\section{Imputation Errors}

Some error, generally expected to be small, is an inevitable result of the various estimations outlined. These imputation errors have not yet been conpletely addressed by ELA and it is possible that estimation mathods may be altered in future surveys. Nationally, 5.9 percent of the crude oil proved reserve estimates, 6.1 percent of the natural gas proved reserve estimates, and 0.7 percent of the lease condensate proved reserve estimates resulted from the imputation and estimation of reserves for those Certainty and Noncertainty operators who did not provide estimates for all of their properties, in combination with the expansion of the sample of Noncertainty operators to the full population. Errors for the latter were guantitatively calculated, as discussed in the previous section. Standard errors, for the former, would tend to cancel each other from operator to operator, and are, therefore, expected to be negligible, especially at the National level of aggregation. In States where a large shate of total reserves is accounted for by Category III and smaller Category II operators, the errors are expected to be somewhat larger than in States where a large share of total reserves is accounted for by Category I and larger Category I operators.

\section{Frame Coverage Errors}

Of all the sources of controllable error connected with the Form ElA-23 survey, errors in the operator frame 
Were expected to be the most important. If the framle does not list all operators in a given State, the sample selected from the frame for the State will not represent the entire operator population, a condition called undercoverage. Undercoverage is a problem wilh certain States, but it does not appear to be a problem with respect to the National proved reserve estimatis for either crude oil or natural gas. While it is relative $y$ straighttorward to use existing sources to identify large operators and find addresses for them, such is not the case for small operators. A frame such as that used :n the 1997 survey is particularly likely to be deficient : States where a large portion of total reserves and production is accounted for by small operators. The:se States are not likely to allocate sufficient resources :o keep rrack of all operators on a current basis. Sorrle undercoverage of this type seems to exist, particularly. with reference to natural gas operators. EIA is continuing to work to remedy the undercoverage problem in those States where it occurred.

\section{Calculation of Reserves of Natural Gas Liquids and Dry Natural Gas}

\section{Natural Gas Liquids Reserve Balance}

The published reserves, production, and reserves change statistics for crude oil, lease conderssate, ard natural gas, wet after lease separation, were derived from the data reported on Form EIA-23 and the application of the imputation methods discussed previously. The information collected on Forn ELA-64A was then utilized in converting the estimates of the wet natural gas reserves into two componenls: plant liquids reserve data and dry natural gas reserve data. The total natural gas liquids reserve estimates presented in Table 14 were computed as the sum of plant liquids estimates (Table 15) and lease condensate (Table 16) estimates.

To generate estimates for each element in the reserves balance for plant liguids in a given producing area, the first step was to group all natural gas processing plants that reported this area as an area-of-origin on their Form EIA-64A, and then sum the liquids production attributed to this area over all respondents. Next, the ratio of the liquids production to the total wet natural gas production for the area was determined. This ratio represented the percentage of the wet natural gas that was recovered as natural gas liquids. Finally, it was assumed that this ratio was epplicable to the reserves and each component of reserve changes (except adjustments), as well as production. Therefore, each element in the wet natural gas reserves balance was multiplied by this recovery tactor to yield the corresponding estimate for plant liguids. Adjustments of natural gas liquids were set equal to the difference between the end of previous year reserve estimates, based upon the current report year Form EIA-23 and Form ELA $64 \mathrm{~A}$ surveys, and the end of current year reserve estimates published in the preceding year's annual reserves report.

\section{Natural Gas Reserve Balance}

This procedure involved downward adjustments of the notural gas data, wet after lease separation, in estimating the volumes of natural gas on a fully dry basis. These reductions were bașed on estimates of the gaseous equivalents of the liquids removed (in the case of production), or expected to be removed (in the case of reserves), from the natural gas stream at natural gas processing plants. Form EIA-64A collected the volumetric reduction, or shrinkage, of the input natural gas stream that resulted from the removal of the NGL at each natural gas processing plant.

The shrinkage volume was then allocated to the plant's reported area or areas of origin. Because shrinkage is, by definition, roughly in proportion to the NGL recovered, $_{r}$ i.e. the NGL produced, the allocation was in proportion to the reported NGL volumes for eact area of origin. However, these derived shrinkage volumes were rejected if the ratio between the shrinkage and the NGL production (gas equivalents ratio) fell outside certain limits of physical accuracy. The ratio was expected to range between 1,558 cubic feet per barrel (where NGL consists primarily of ethane) and 900 cubic feet per barrel (where NGL consists primarily of natural gasolines). When the computed gas equivalents ratio fell outside these limits, an imputed ratio was utilized to estimate the plant's natural gas shrinkage allocation to each reponted area of origin.

This intputed ratio was that calculated for the aggregate of all other plants reporting production and shrinkage, and having a gas equivalent ratio within the aforesaid limits, from the area in question. The imputed area ratio was applied only if there were at least tive plants to base its computation on. If there were less than tive plants, the imputed ratio was calculated based on all plants in the survey whose individual gas equivalents ratio was within the acceptable limits. Less than one percent of the liguids production was associated with shrinkage volumes 
imputed in this manner. Based on the 1997 Form EIA-64A survey, the national weighted average gas equivalents ratio was computed to be 1,392 cubic teet of natural gas shrirkage per barrel of NGL recovered. The total shrirkage volume (reported plus imputed) for all plants reporting a given area of origin was then subtracted from the estimated value of natural gas production, wet after lease separation, yielding dry natural gas production for the area. The amount of the reduction in the wet natural gas production was then expressed as a percentage of the wet natural gas production. Dry natural gas reserves and reserve changes were determined by reducing the wet natural gas reserves and reserve changes by the same percentage reduction factor.

A further refinement of the estimation process was used to generate an estimate of the natural gas liquids reserves in those States with coalbed methane fields. The States where this procedure was applied were Alabama, Colorado, Kansas, New Mexico, Oklahoma, Pennsylvania, Utah, Virginia, West Virginia, and Wyoming. The first step in the process was to identify all Form ELA-23 reported coalbed methane fields. The assumption was made that coalbed methane fields contained little or no extractable natural gas liquids.
Therefore, when the normal shrinkage procedure was applied to the wet gas volume reserve components, the estimate of State coalbed methane volunes were excluded and were not reduced for liquid extraction. Following the computation for shrinkage, each coalbed field gas volume reserve components was added back to each of the dry gas volume reserve components in a State. The effect of this is that the lange increases in reserves in some States from coalbed methane fields did not cause corresponding increases in the State natural gas liquids proved reserves.

Adjustments of dry natural gas were set equal to the difference between the end of previous year reserves estimates, based upon the current report year Form EIA-23 and Form EIA-64A surveys, and the end of current year reserve estimates published in the preceding year's arnual reserves report.

Each estimate of end of year reserves and report year production has associated with it an estimated sampling etror. The standard errors for dry natural gas were computed by multiplying the wet natural gas standard errors by these same percentage reduction factors. Table $\mathbf{F 7}$ provides estimates for 2 times the S. E. $\left(\hat{V}_{s}\right)$ for dry natural gas. 

Appendix $G$

Estimation of Reserves and Resources 



\section{Estimation of Reserves and Resources}

\section{OIl and Gas Resource Base}

Universally accepted definjtions have not been developed for the many terms used by geologists, engineers, accountants and others to denote various components of overall oil and gas resources. In part, this is because most of these terms describe estimated and therefore uncertain, rather than measured, quantities. The lack of standardized terminology sometimes leads to inaccurate understanding of the meaning and/or import of estimates. Particularly common is an apparently widespread lack of understanding of the substantial difference between the terms "reserves" and "resources", as indicated by the frequent misuse of either term in place of the other.

The total resource base of oil and gas is the entire volume formed and trapped in-place within the Earth before any production. The laggest portion of this total resource base is nonrecoverable by current or foreseeable technology. Most of the nonrecoverable volume occurs at very low concentrations throughout the earth's crust and cannot be extracted short of mining the rock or the application of some other approach that would consume more energy than it produced. An additional portion of the total resource base cannot be recovered because cutrently available production techniques cannot extract all of the in-place oil and gas even when present in commercial concentrations. The inability to recover all of the in-place oil and gas from a producible deposit occurs because of unfavorabte economics, intractable physical forces, or a combination of both. Recoverable resources, the subset of the total resource base that is of societal and economic interest, are defined so as to exclude these nonuecoverable portions of the total resource base.

The structure presented in Figure G1 outlines the total resource base and its components. The total resource base first consists of the recoverable and nonrecoverable portions discussed above. The next level down divides recoverable resources into discovered and undiscovered segments. Discovered resources are further separated into cumulative (i.e., all

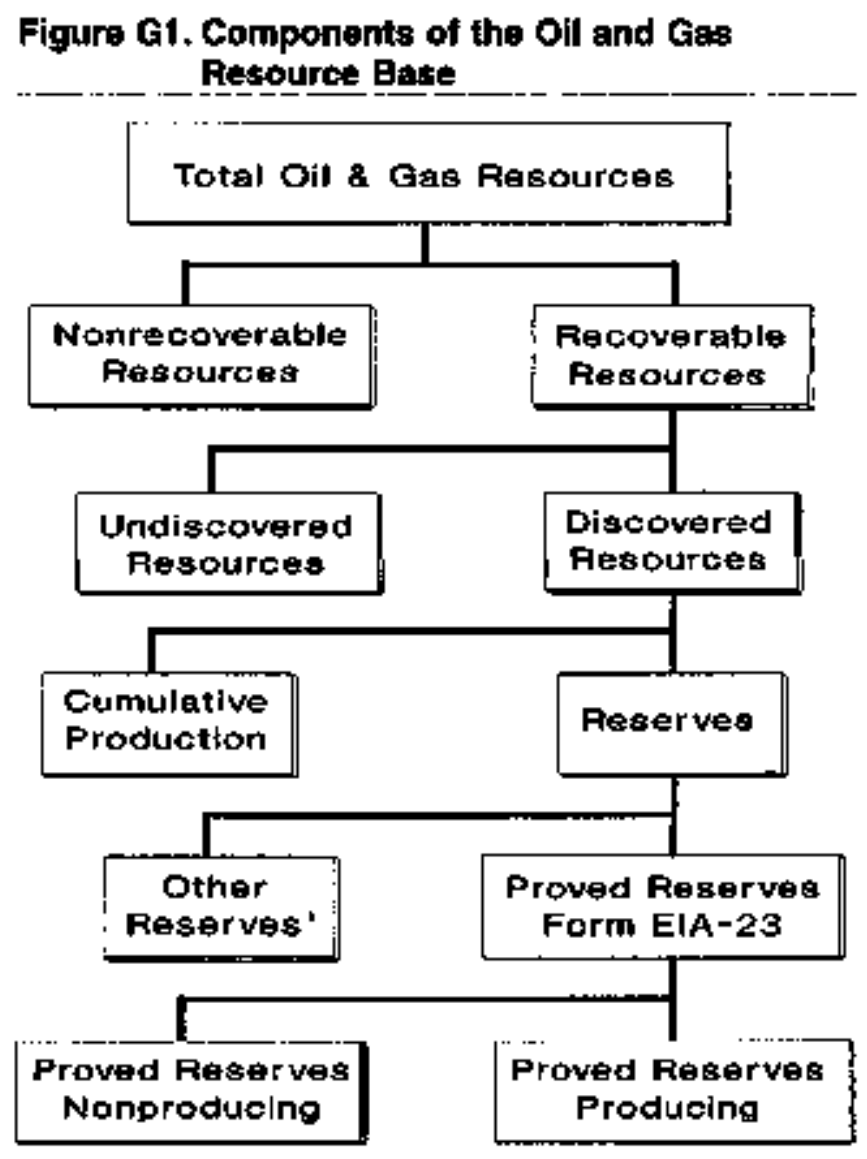

'Of the numerous other roserve dassifications, onhy "indiceled
Additonal nesserves are included in this report. Souras: Energy Intomation Administration, Olice of $\mathrm{O}$ a and Gas.

past) production, and reserves. Reserves are additionally subdivided into proved reserves and "other reserves".

\section{Recoverable Resources}

Discovered recoverable resources are those economically recoverable quantities of oil and gas for which specific locations are known. While the specific locations of estimated undiscovered recoverable resources are not yet known, they are believed to exist in geologically favorable settings. 
Current estimates of undiscovered recoverable resources merit discussion in order to provide a useful sense of scale relative to proved reserves. The sources of official estimates of domestic undiscovered recoverable resources are two agencies of the Department of the Interior (DOI), the United States Geological Survey (USGS) for onshore areas and those offshore waters subject to State jurisdiction, and the Minerals Management Service (MMS) for those offshore waters under Federal jurisdiction.

The USGS defines undiscovered recoverable conventional resources as those expected to be resident in accumulations of sufficient size and quality that they could be produced using convertional recovery technologies, without regard to present econorric viability. Therefore, only part of the USGS undiscovered recoverable conventional resource is economically recoverable now. The USGS also defines a class of resources that occut in "continuous-type" accumulations. Unlike conventional oil and gas accumulations, continuous-type accumulations do not occur in discrete reservoirs of limited areal extent. They include accumulations in low-permeability (tight) sandstones, shales, and chalks, and those in coal beds. Again, only part of the continutous-type technically recoverable resource is economically recoverable no $\mathrm{W}$. In fact, only a small portion of the in-plaze continuous-type resource accumulations are estimated to be techniçally recoverable now. Table G1 presents the latest available USGS and MMS estimates, along with the EIA 1997 proved reserves estimates.

Technically recoverable resources of wet natural gas (discovered, both proved and unproved, and undiscovered) are estimated at 1,341 trillion cubic feet (Table G1). Subtracting U.S. proved reserves of 175 trillion cubic feet yields an unproven technically recoverable resource target of 1,166 trillion cubic feet. This is about 61 times the 1997 gas production level.

Other organizations have also estimated unproven technically recoverable gas resources. For example, tue Potential Gas Committee (PGC), an industry sponsored group, provides detailed geology-based gas resource estimates every 2 years. In 1996 the PGC mein estimate of potential gas resources was $1_{r} 067$ trillion cubic feet, about 99 trillion cubic feet less than the DOI estimates in Table G1. Arother recent estimate was made by the National Petroleum Council (NPC), in industry-based group that serves in an advisory capacity to the US. Secretary of Energy. The NPC's estimate, based on data available at year-end 1990, was 1,135 trillion cubic feet, 111 trillion cubic feet less thin the DOI estimates summarized in Table G1. The differences among these estimates are usually due to the availability of newer data, the differences in coverage or resource category definitions, and to legitimate but differing data interpretations. The USGS estimates of reserve growth in known fields are much larger than previous estimates due to the utilization of newer EIA reserves growth data.

While the estimation of undiscovered teșourçes is certainly a more imprecise endeavor than is the estimation of proved reserves, it is clear that substantial volumes of technically recoverable oil and gas resources remain to be found and produced domestically. Current estimates indicate that as much domestic gas remains to be found and then produced as has been to date. Of course, much effort, investment and time wrill be required to bring this gas to market.

There is a perception that the oil resource base has been more intensively developed than the gas resource base. And in fact, more oil has been produced in the United States than is estimated as remaining recoverable. Nevertheless, the ratio of 1996 unproven technically recoverable oil resources to oil production (Table Gi) was about 62 to 1 , higher than the comparable gas ratio.

\section{Discovered Resources}

In addition to cumulative production, which is the sum of current year production and the production in all prior years, estimates of discovered recoverable resources include estimates of reserves. Broadly, teserves are those volumes that are believed to be recoverable in the future from known deposits through the eventual application of present or anticipated technology.

\section{Regerves}

Reserves include both proved reserves and other reserves. Several different reserve elassification systems are in use by different organizations, as preferred for operational reasons. These systems utilize and incorporate various definitions of terms such as measured reserves, indicated reserves, inferred reseroes, probable reserves, and possible reserves. As used by the diffetent organizations, the definitions that attach to these terms sometimes overlap, or the terms may require a slightly different interpretation from one organization to the next. Nevertheless, all kinds of "other reserves" are generally less well known and therefore less precisely quantifiable than proved reserves, and their eventual recovery is less assured. 
Table G1. Estimated Oil and Gas Reserves and Mean Estlmates of Technically Recoverable Oil and Gas Resources

\begin{tabular}{|c|c|c|c|}
\hline Catregories & $\begin{array}{c}\text { Crude Oil" } \\
\text { (million barridg) }\end{array}$ & $\begin{array}{l}\text { Natural Gas (Dry) } \\
\text { (billan cubic fefif) }\end{array}$ & $\begin{array}{l}\text { Matuzal Gies } \\
\text { Liquids } \\
\text { (million bairela) }\end{array}$ \\
\hline \multicolumn{4}{|l|}{ Lower 48 States } \\
\hline \multicolumn{4}{|l|}{ Discovered } \\
\hline Proved Reserves \{ElA, 1997) . . . . . . . . . . . . . . . . . . . . & 17,385 & ${ }^{6} 156,661$ & 7,342 \\
\hline Reserve Growth - conventiossal, onshore ${ }^{\circ}$ (USGS, 1991) . . . . . . . & $d_{47,000}$ & 290,000 & 12,900 \\
\hline Reserve Growth - conventional, Federal Offshore (MMS, 1995).... & ${ }^{6} 2,238$ & 32,719 & NE \\
\hline Unproved Reserves, Federal Offshore (MMS, 1996) ............ & 1,643 & 4,436 & NE \\
\hline \multicolumn{4}{|l|}{ Undiscovered, Tecthnictally Recoverable } \\
\hline Conventional, onshore ${ }^{c}$ (USGS, 1993) $\ldots \ldots \ldots \ldots \ldots \ldots \ldots$ & 21,810 & 190,280 & 6,080 \\
\hline Conlinuous-1ype - sanctsione, shiag, chalk; onshore ' (USGS, 1993). & 2,066 & $308,0 \mathrm{do}$ & 2,119 \\
\hline Cominuous type - coalbeds, onshore ${ }^{c}$ (USGS, 1993) . . . . . . . . . & & 49,910 & \\
\hline Federal Offshore - conventional (MMMS, 1994). . . . . . . . . . . . & 21,300 & 142,100 & $f_{<1,800}$ \\
\hline Subtotal $\ldots \ldots \ldots \ldots \ldots \ldots \ldots \ldots \ldots \ldots \ldots \ldots \ldots \ldots$ & 113,442 & $1,174,196$ & MA \\
\hline \multicolumn{4}{|l|}{ Alaska } \\
\hline \multicolumn{4}{|l|}{ Discoovered } \\
\hline Proved Reserves (E|A, 1997) . . . . . . . . . . . . . & 5,161 & 10,673 & 631 \\
\hline Feserve Growth - conventional, onshore ${ }^{\circ}$ (USGS, 1991) , . . . . . & $9,3,000$ & 32,000 & 500 \\
\hline Reserve Growth corwemtional, Federal Offshore (MMS, 1994). .... & 0 & 0 & NE \\
\hline Unproved Reserves, Federal Offshore (MMS, 1994) . . . . . . . . . & 400 & 700 & NE \\
\hline \multicolumn{4}{|l|}{ Undiscossered, Technically Recoverable } \\
\hline Conventional onshore ${ }^{C}$ iUSGS, 1993$) \ldots \ldots \ldots \ldots \ldots \ldots \ldots$ & 8,440 & 68,410 & 1,120 \\
\hline Continuous-type - sendstone, shale, chalk; onshore ${ }^{C}$ (USGS, 1993). & NE & NE & NE \\
\hline Continuous-type - coalbeds, onshore ${ }^{c}$ (USGS, 1993) . . . . . . . . & & NE & NR \\
\hline Fetieral Offshore - conventional (MMS, 1994) . . . . . . . . . . . . & 24,300 & 125,900 & $<<1,800$ \\
\hline Subtotal $+\ldots+\ldots \ldots \ldots \ldots \ldots \ldots \ldots \ldots \ldots \ldots \ldots \ldots \ldots \ldots \ldots$ & 51,301 & 237,683 & Na \\
\hline Total Lower 4 s States and Alaske. $\ldots \ldots \ldots \ldots \ldots \ldots \ldots \ldots \ldots$ & 164,743 & $1,411,069$ & 32,492 \\
\hline \multicolumn{4}{|l|}{ Deductions for Production and Proved Reserves Changes. } \\
\hline 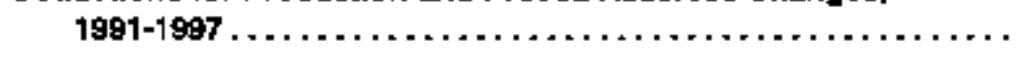 & $-10,290$ & $-80,680$ & $-4,014$ \\
\hline U.S. Total, $1997, \ldots \ldots \ldots \ldots \ldots \ldots \ldots$ & 154,444 & $1,330,969$ & $28,47 a$ \\
\hline
\end{tabular}

\footnotetext{
Condensale is includted with crudo cil for MASS ețtimates In Federal OHtahore reglons.

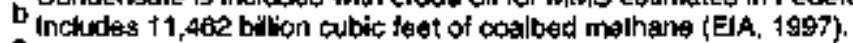

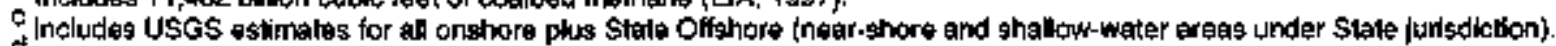

of Using USGS definition, 1,924 millon banels of indicaled additional oil reserves in the lower 48 States were included (EIA, 1998).

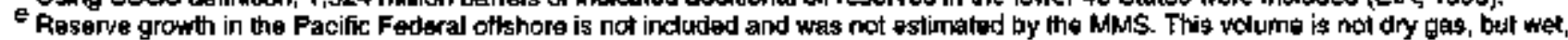
atter lese geparation.
}

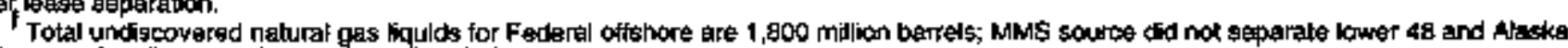
aslimates of undiscovered nahural gas $\|$ quide (19B6).

9 Using USBS definition. 952 milition barreats of indicaled addillonel oil reserves in Aleska were included (ElA, 1998).

NE = nol es: imated.

NA = nol applicable.

Notes: Federal Offshore Indicates MMS estimales for Federal Ofishore Jurizdictions tOuter Continental Shell apd deeper water areas

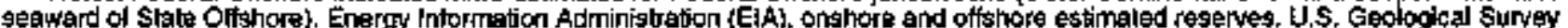

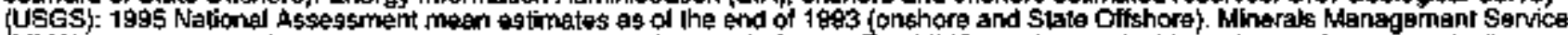

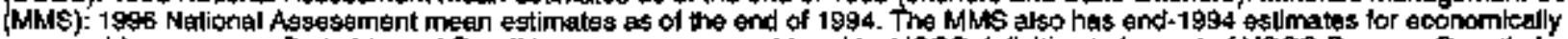
recowerable resources. Probeble and Possible reserves are conshderad by USGS deflnition to be part or USGS Aeserve Growth, but gre

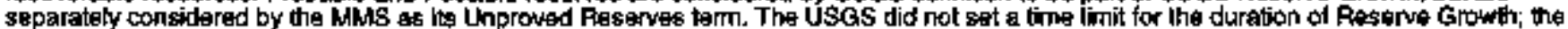
Whis set the year 2020 as the lime limil in its estlmales of Reserve Growth in existing fields of lhe Gulf of hilexios. Exctuded irom the estimales are undlscovered oil resources in tar deposits and oil sheles, and undiscovered gas resolurces in geopressured brinas and gas bydrates.

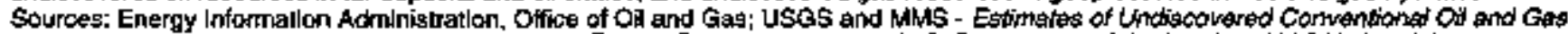

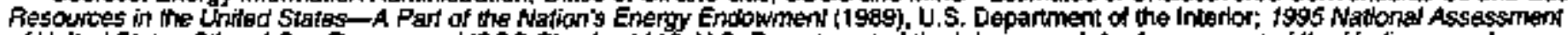

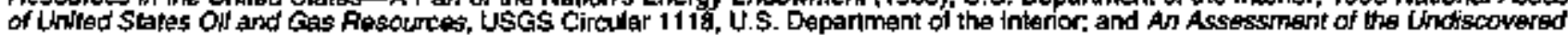
Hydrocarbon Potential of the Nablon's Outer Comthental Sheff $\{1996\}$, U.S. Department of the Interior. 
Measured reserves are defined by the USCS as that part of the identified (i.e., discovered) economically recoverable resource that is estimated from geologi:evidence and supported directly by engineerings data. $\{1\}$ They are similarly defined by the MMS, although its system also subdivides them by degree of development and producing status. $\{42\}$ Measured reserves are demonstrated with reasonable certainty to be recoverable in future years from known reservoirs under existing economic and operating conditions, and are essentially equivalent to proved reserves as defined by the EIA. Effectively, estimates of proved reserves may be thought of as reasonable estimates (as opposed to exact measures) of "on-the-shelf inventory"

Inferred reserves and indicated reserves, due to their more uncertain economic or technical recoverability, are included in the "other reserves" category. The USGS defines inferred reserves as that part of the identified economically recoverable resource, over and above both measured and indicated (see below) reserves, that will be added to proved reserves in the future through exterssions, revisions, and the discovery of new pay zones in already discovered fields. $\mid 41$ | Inferred reserves are considered equivalent to "probable reserves" by many analysts, for exampli, those of the PGC.

Indicated additional reserves, a separate category, are defined by both the DOI and the ELA as quantities of crude oil that may become economically recoverable in the future from existing productive reservoirs through the application of currently available but as-yet urinstalled recovery technology. At such time as the technology is successfully applied, indicatet additional reserves are reclassified to the provet reserves category. Of all the various "other reserves" categories, only indicated additional reserves are estimated by the EIA and reported herein.

\section{Proved Reserves}

The EIA defines proved reserves as those volumes of oil and gas that geological and engineering data demonstrate with reasonable certainty to be recoverable in future years from known reservoirs under existing economic and operating conditions.

When deterministic proved reserves estimation methods are used, the term reasonable certainty is intended to express a high degree of confidence that the estimated quantities will be recovered. When probabilistic methods are used there should be at leasit a 90 percent probability that the actual quantities recovered will exceed the estimate.

Proved reserves are either proved producing or proved nonproducing (i.e, resident in reservoirs that did not produce duting the report year). The latter may represent a substantial fraction of total proved resserves.

\section{Reserve Estimation Methodologies}

The adoption of a standard defintion of proved reserves for each type of hydrocaibon surveyed by the Form EIA-23 program provided a far more consiskent response from operators than if each operator had used their own definition. Such standards, however, do not guarantee that the resulting estimates themselves are determinate. Regardless of the definition sejected, proved reserves cannot be measured directly. They are estimated quantities that are inferred on the basis of the best geological, engineering, and economic data available to the estimator, who generally uses considerable judgment in the analysis and interpretation of the data. Consequently, the accuracy of a given estimate varies with and depends on the quality and quantity of raw data available, the estimation method used, and the training and experience of the estimator. The element of judgment commonly accounts for the differences among independent estimates for the same reservoir or field.

\section{Data Used in Making Reserve Estimates}

The raw data used in estimating proved reserves include the engineering and geological data for reservoir rock and its fluid content These data are obtained from direct and indirect measurements. The data available for a given reservoir vary in kind, quality, and quantity. When a reservoir is ftrst discovered only data from a single well are available, and prior to flow testing or actual production, proved reserves can only be inferred. As development of the reservoir proceeds, and flow tests are made or actual production commences, more and more data become available, enabling proved reserves estimates to become more accurate.

Many different kinds of data are useful in making reserves estimates. They may include: data on porosity, permeability, and fluid satusations of the reservoir 
rocks (obtained directly from core analysis or from various types of electrical measurements taken in a well or several wells); data on the production of fluids from a well or several wells; geologic maps of the areal extent, thickness, and continuity of the reservoir rocks (inferred from well Jogs, geophysical, and geological data); and reservoir pressure and temperature data. Also involved are economic data including the current price of crude oil and natural gas, and various developmental and operating costs.

\section{Reserve Estimation Techniques}

Depending on the kinds and amounts of data available, and a judgment on the reliability of those datar the estimator will select one of several methods of making a proved reserves estimate. Methods based on production performance data are generally more accurate than those based strictly on inference from geological and engineering data. Such methods include the Praduction Decline method (for crude oil or natural gas reservoirs), the Material Balance method (for crude oil reservoirs), the Pressure Decline method (which is actually a material balance, for natural gas reservoirs), and the Resertoir Simulation method (for crude oil or natural gas reservoirs). The reservoir type and production mechanisms and the rypes and amounts of reliable data available determine which of these methods is more appropriate for a given reservoir. These methods are of comparable accuracy.

Methods not based upon production data include the Volumetric method (for crude oil or natural gas reservoirs) and the Nominat method. Of these, the Volumetric method is the more accurate. Both methods, however, are less accurate than those based on production data. Table $\mathbf{G 2}$ summarizes the vatious Inethods.

\section{Judgmental Factors in Reserve Estimation}

The determination of rock and hydrocarbon fluid properties involves judgment and is subject to some uncertainty; however, the construction of the geologic maps and cross sections and the determination of the size of the reservoir are the major judgmental steps in the Volumetric method, and are subject to the greatest uncertainty. Estimates made using the Moterial Balance method, the Reserooir Simulation method, or the Pressure Decline method are based on the simator's judgment that the type of reservoir drive mechanism
Table G2. Feserve Estimation Technlques

\begin{tabular}{|c|c|}
\hline Method & Comments \\
\hline Volumetric & $\begin{array}{l}\text { Applies to cruda oil and natural gas teser- } \\
\text { voirs. Eased on raw engineering and geo- } \\
\text { loglc data. }\end{array}$ \\
\hline Malerial Balance & $\begin{array}{l}\text { Applies to crude oil and natural gas reser- } \\
\text { woirs. bs used in eslimating reserves. Usu- } \\
\text { ally of more value in predicting reserves, } \\
\text { and reservoir perfonmance. }\end{array}$ \\
\hline Pressure Decline & $\begin{array}{l}\text { Applies to monagsociated and associated } \\
\text { gas reserwolts. The method is a special } \\
\text { case of molerlal balance equation in the } \\
\text { absence of water intlux. }\end{array}$ \\
\hline Production Decline & $\begin{array}{l}\text { Applies } 10 \text { crude ofl and natural gas reser- } \\
\text { woirs during production dedine (usually in } \\
\text { the laler stages of reservoir life). }\end{array}$ \\
\hline Reserwoir Simulatlo & $\begin{array}{l}\text { nApplies to crude coll and natural gas reser- } \\
\text { woirs. is used in estimeting reserves. Usu- } \\
\text { ally of more value in predicling reservoir } \\
\text { performance. Accuracy incleases when } \\
\text { matched with past pressure and produc- } \\
\text { lion data. }\end{array}$ \\
\hline Nominal & $\begin{array}{l}\text { Applied lo cnude ofl and natural gas reser- } \\
\text { woirs. Based on rule of thumb or anakgy } \\
\text { with another reservolr or reserwoirs be- } \\
\text { leved to be similar; leasl accurate ol } \\
\text { methods used. }\end{array}$ \\
\hline
\end{tabular}

has been identified and on the specification of abandonment conditions. Estimates based on the Production Decline method are subject to judgment in constructing the trend line, and are based on the estimator's assumption of reservoir performance through abandonment.

Contributing to the degree of uncertainty inherent in the above methods for estimating reserves are other factors associated with economic considerations and the perceived reservoir limits, which together influence the final reserves estimate. A brief discussion of these other factors follows.

Economic considerations: There has been continuing debate about the effects of prices on proved reserves. Although no all-inclusive statement can be made on the impact of price, the points at issue can be discussed and some general remarks can be made about some circumstances where price may be a factor.

- Deceloped gas fuelds - In a gas reservoit, price affects the economic limit (i.e., the production rate required to meet operating costs) and, therefore, the abandonment pressure. Thus, price change has some effect on the conversion of 
nonecononic hydrocarbon resources to the category of proved reserves. In both nearly depleted reservoirs and newly developed reservoirs, the actual increase in the quantity of proved reserves resulting from price rises is generally limited in terms of national volumes (even though the percentage increase for a given reservoir may be great).

- Developed ofl fields - In developed crude oil reservoirs many of the same comments apply; however, there is an additional consideration. If the price is raised to a level sufficient to justify initiation of an improved recovery project, and if the improved recovery techuique is effective, then the addition to ultimate recovery from the reservoir can be significant. Because of the speculative nature of predicting prices and costs many years into the future, proved reserves are estimated on the basis of current prices, costs, and operating practices in effect as of the date the estimation was made.

- Successful exploration efforts - Price can have a major impact on whether a new discovery is produced or abandoned. For example, the decision to set casing in a new onshore discovery, or to install a platiorm as the result of an offshore discovery, are both price-sensitive. If the decision is made to set pipe or to install a platform, the discoveries in both cases will add to the proved reserves total. If such projects are abandoried, they will make no contribution to the proved reserves total.

Effect of operating conditions: Operating conditions are subject to change caused by changes in economic conditions, unforeseen production problems, new production practices or methods, and the operator's financial position. As with economic conditions, operating conditions to be expected at the time of abandonment are speculative. Thus, current operating conditions are used in eștimating proved reserves. In considering the effect of operating conditions, a distinction must be made between processes and techniques that would normally be applied by a prudent operator in producing his oil and gas, and initiation of changes in operating conditions that would require substantial new investment.

- Compression - Compression facilities are normally installed when the productive capacity or deliverability of a natural gas reservoir or is individual wells dectines. In other cases compression is used in producing shallow, low-pressure reservoirs or reservoirs in which the pressure has declined to a level too low for the gas to flow into a higher pressure pipeline.
The application of compression increases the pressure and, when economical, is used to make production into the higher pressure pipeline possible. Compression facilities normally require a significant investment and result in a change in operating conditions. It increases the proved reserves of a reservoir, and reasonably accurate estimates of the increase can be made.

- Well stimulation - Procedures that increase productive capacity (workovers, such as acidizing or fracturing, and other types of production practices) are routine field operations. The procedures accelerate the rate of production from the reservoir, or extend its life, and they have only strall effect on proved reserves. Reasonable estimates of their effectiveness can be made.

- Improved recovery techniques - These techniques involve the injection of a fluid or fluids into a zeservoir to augment natural reservoir energy. Because the response of a given reservoir to the application of an improved recovery technique cannot be accurately predicted, crude oil production that may ultimately result from the application of these techniques is classified as "indicated additional reserves of cude oil" rather than as proved reserves until response of the reservoir to the technigue has been demonstrated. In addition, improved recovery methods are not applicable to all crude oil reservoirs. Initiation of improved recovery techuiques may require significant investment.

- Infill driling - Infill drilling (drilling of additional wells within a field/reservoir) may result in a higher recovery factor, and, therefore, be economically justified. Predictions of whether infill drilling will be justified under current economic conditions are generally based on the expected production behavior of the infill wells.

Reservoir limits: The initial proved reserves estimate made from the discovery well is subject to significant uncertainty because one well provides little information on the size of the resservoir. The area proved by a discovery well is frequently estimated on the basis of experience in a given producing region. Where there is continuity of the producing formation over wide geographic areas, a relatively large proved area may be assigned. In some cases where reliable geophysical and geological data are avajlable, a reasonable estimate of the extent of the reservoir can be made by drilling a relatively small number of delineation wells. Conversely, a relatively small proved area may be assigned when the producing 
formation is of limited continuity, owing to either ștructural or lithological factors.

Additional wells provide more information and reduce the uncerkainty of the reserves estimate. As additional wells axe drilled, the geometry of the reservoir and, consequently, its bulk volume, become more clearly defined. This process accounts for the large extensions to proved reserves typical of the early stages of most reservoir development. 

Appendix $\mathrm{H}$

Maps of Selected State Subdivisions 


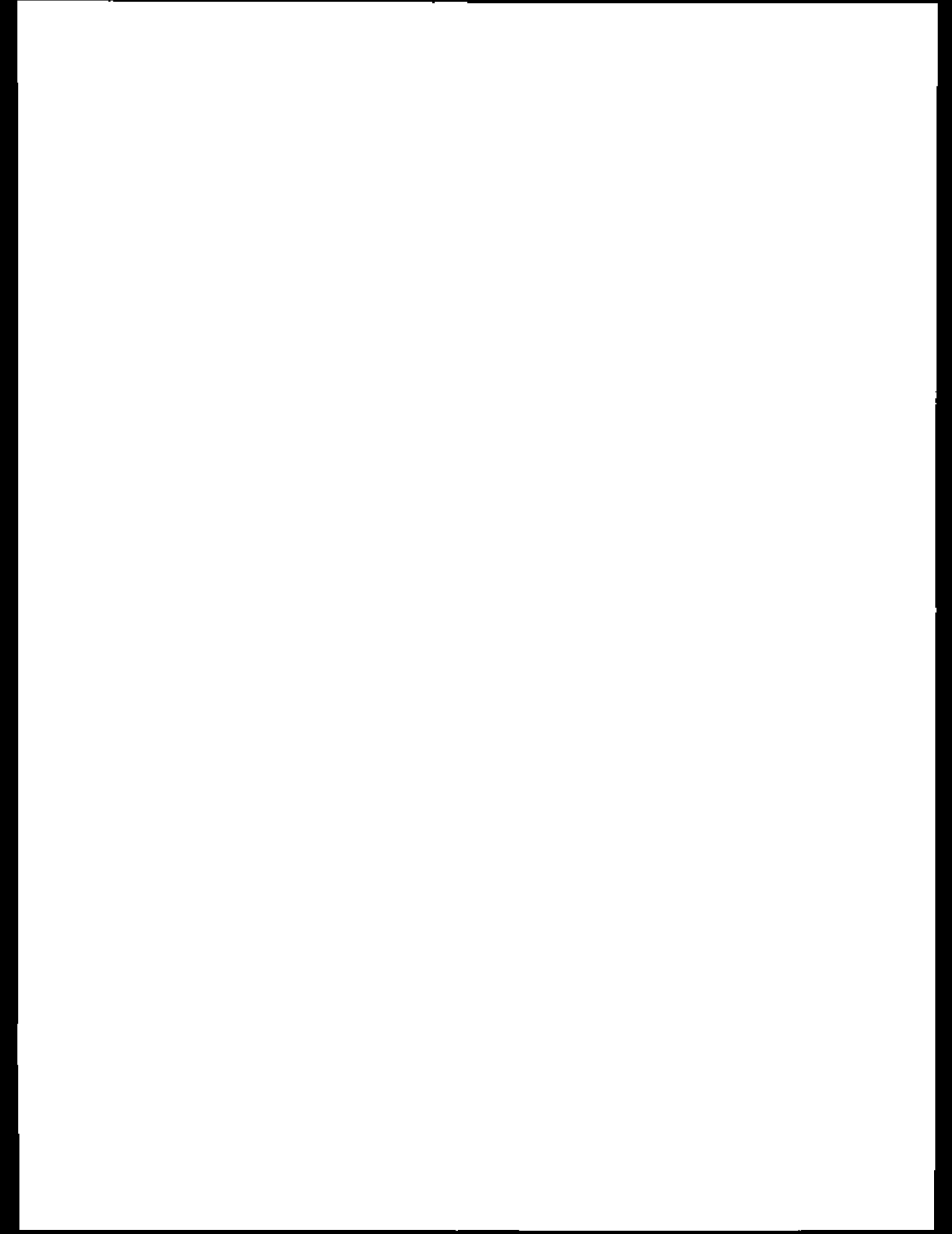




\section{Appondix $\mathbf{H}$}

\section{Maps of Selected State Subdivisions}

Figure H1. Subdivisions of Alaska

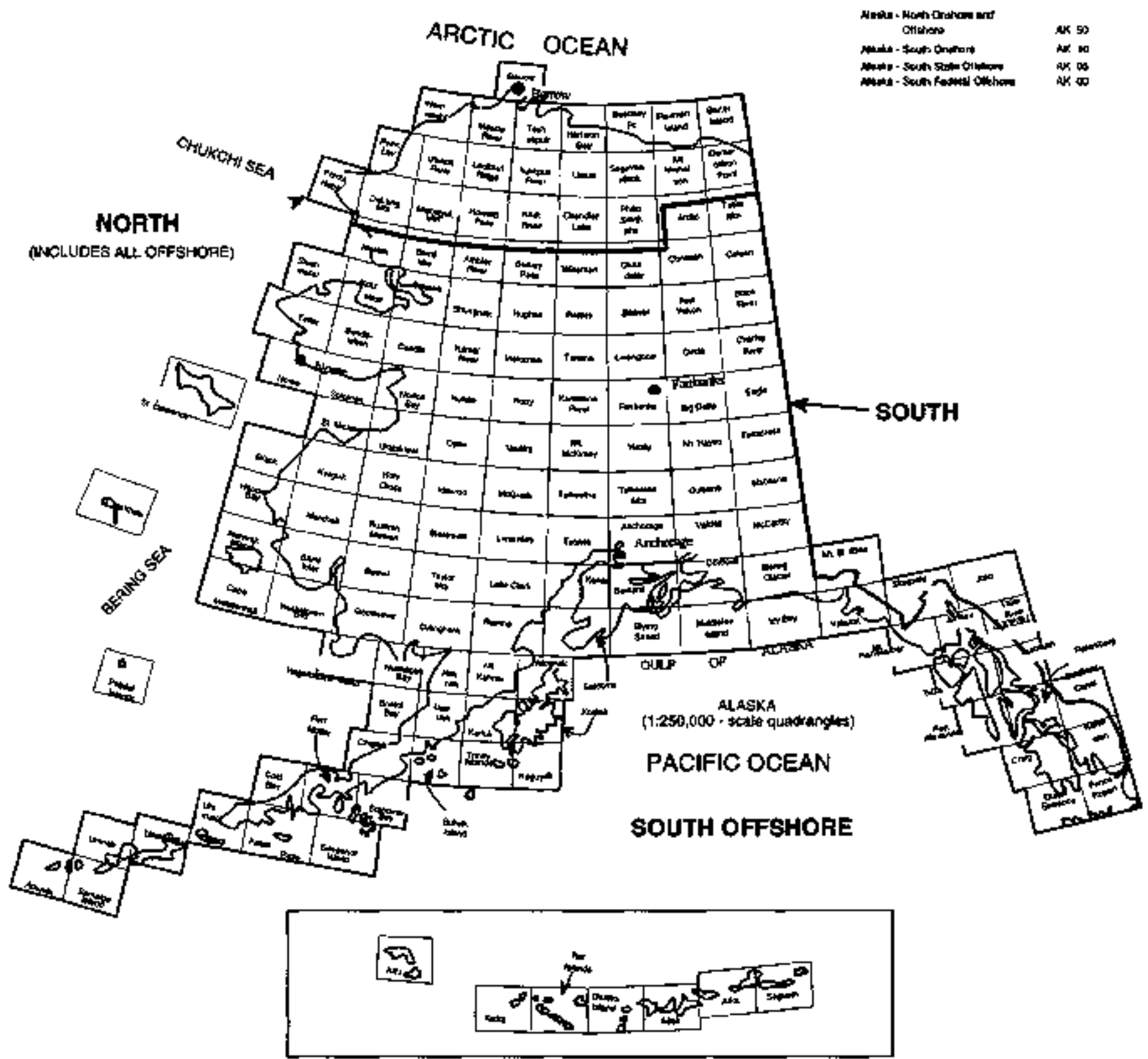

Source: After L.S. Geological Survey. 
Figure H2. Subdivisions of Callfornla

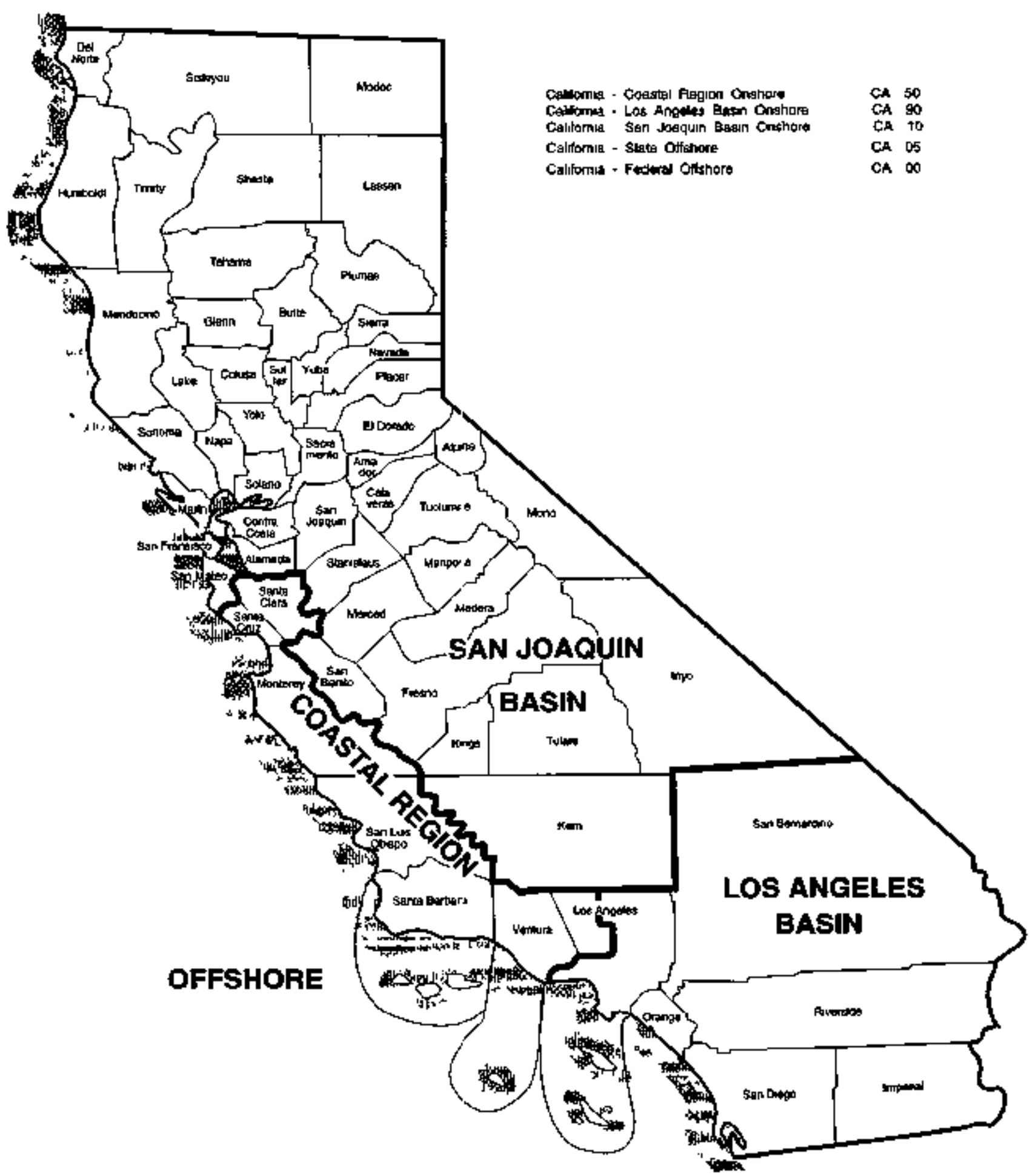

Source Energy Information Adrninistrabon, Offce of OU arkl Eas 
Figure H3. Subdivisions of Louisiana

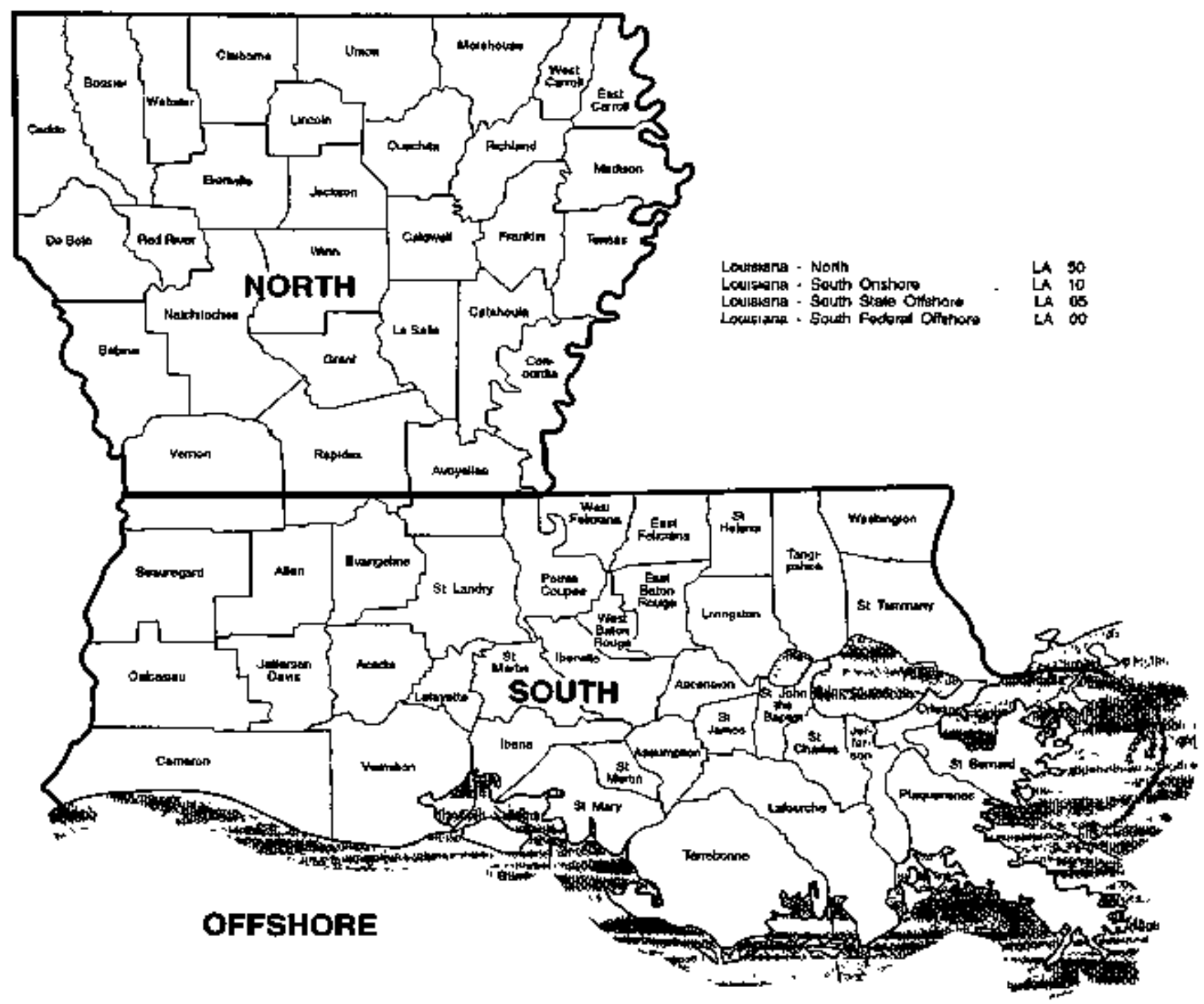

Source: Energy Intarmalion Administration. Otfice of Oil and Ges. 
Flgure H4. Subdivisions of New Mexico

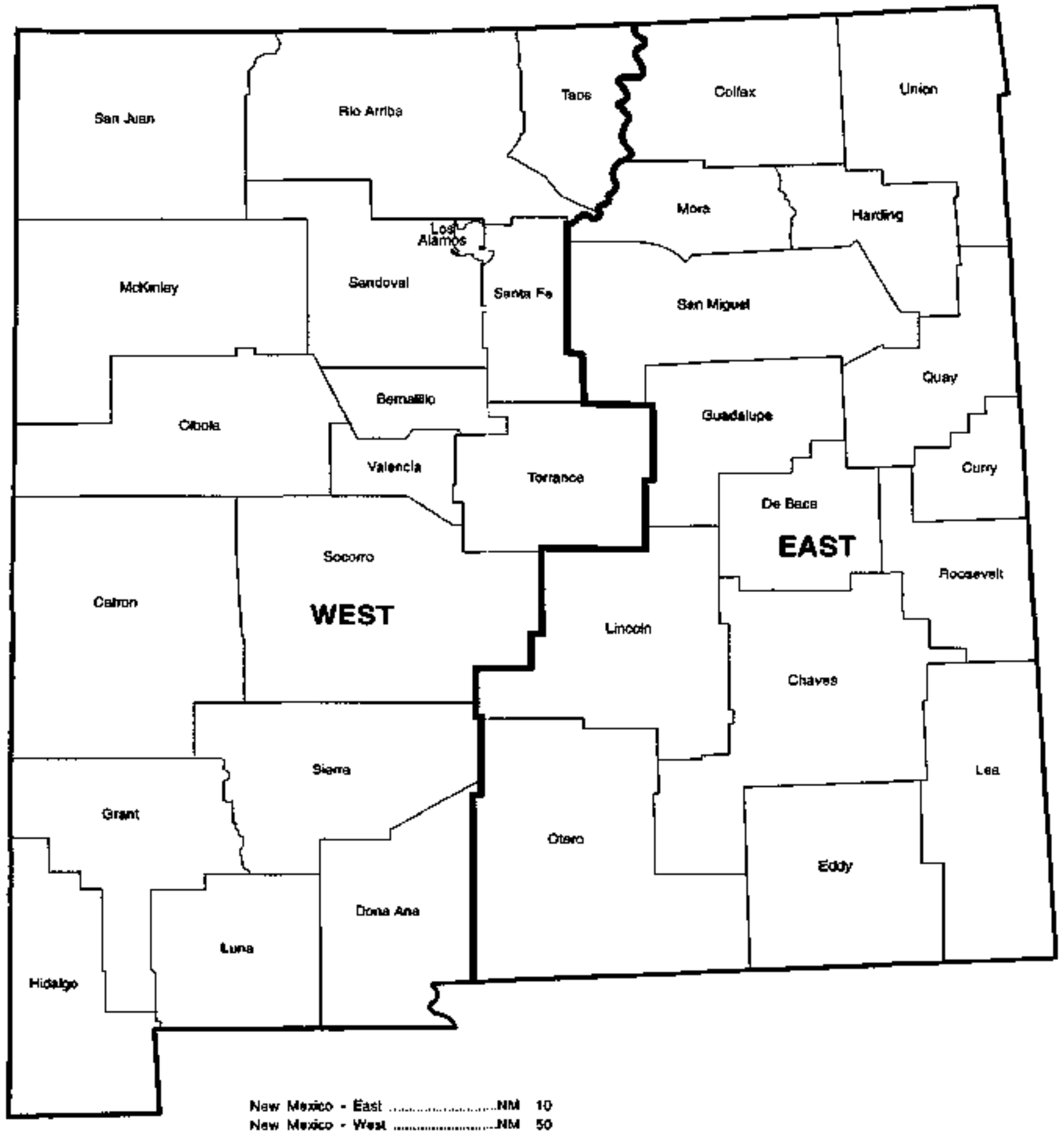

Source: Enargy Iniommation Adrinistration, Office of Oil grel Gas. 


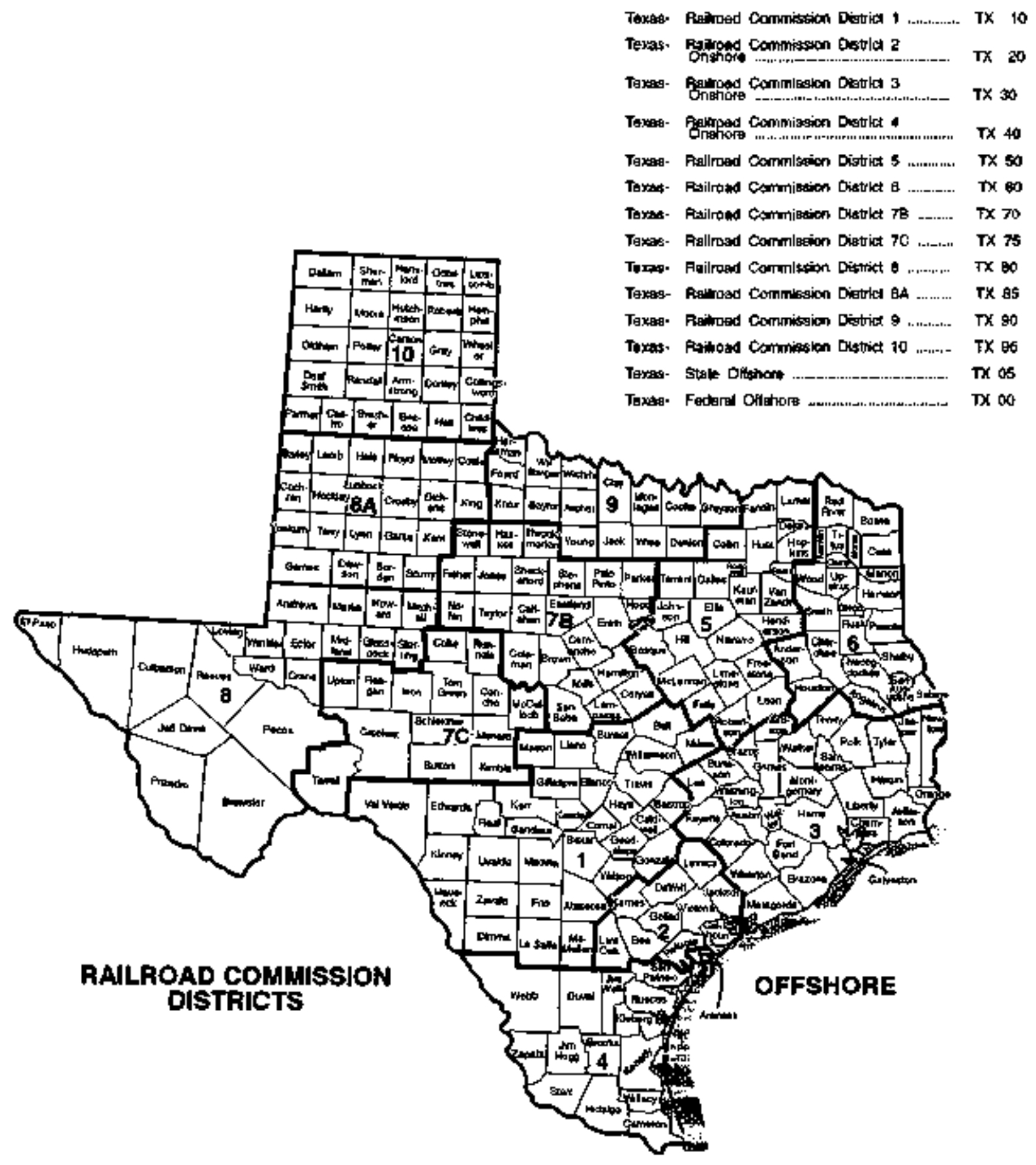

Sarta: Energy Information Admirlstration, Ofice of OAl and Gas. 
Figure H6. Westem Planning Area, Gulf of Mexico Outer Continental Shelf Region

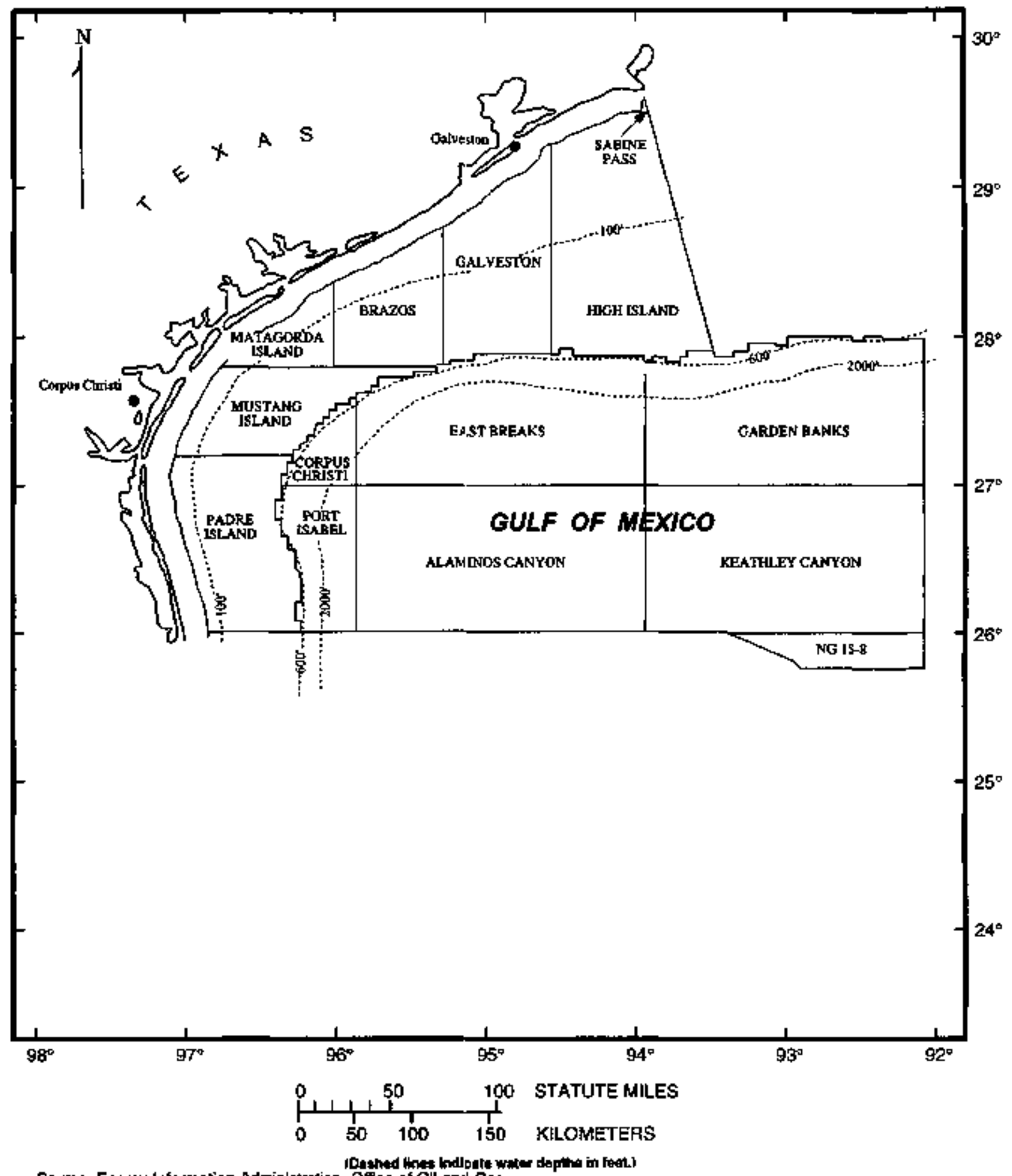

Sounso: Energy Information Administration, Ofilee of Oll and Gas 
Flgure H7. Central Planning Area, Gulf of Mexico Outer Continental Shelf Region

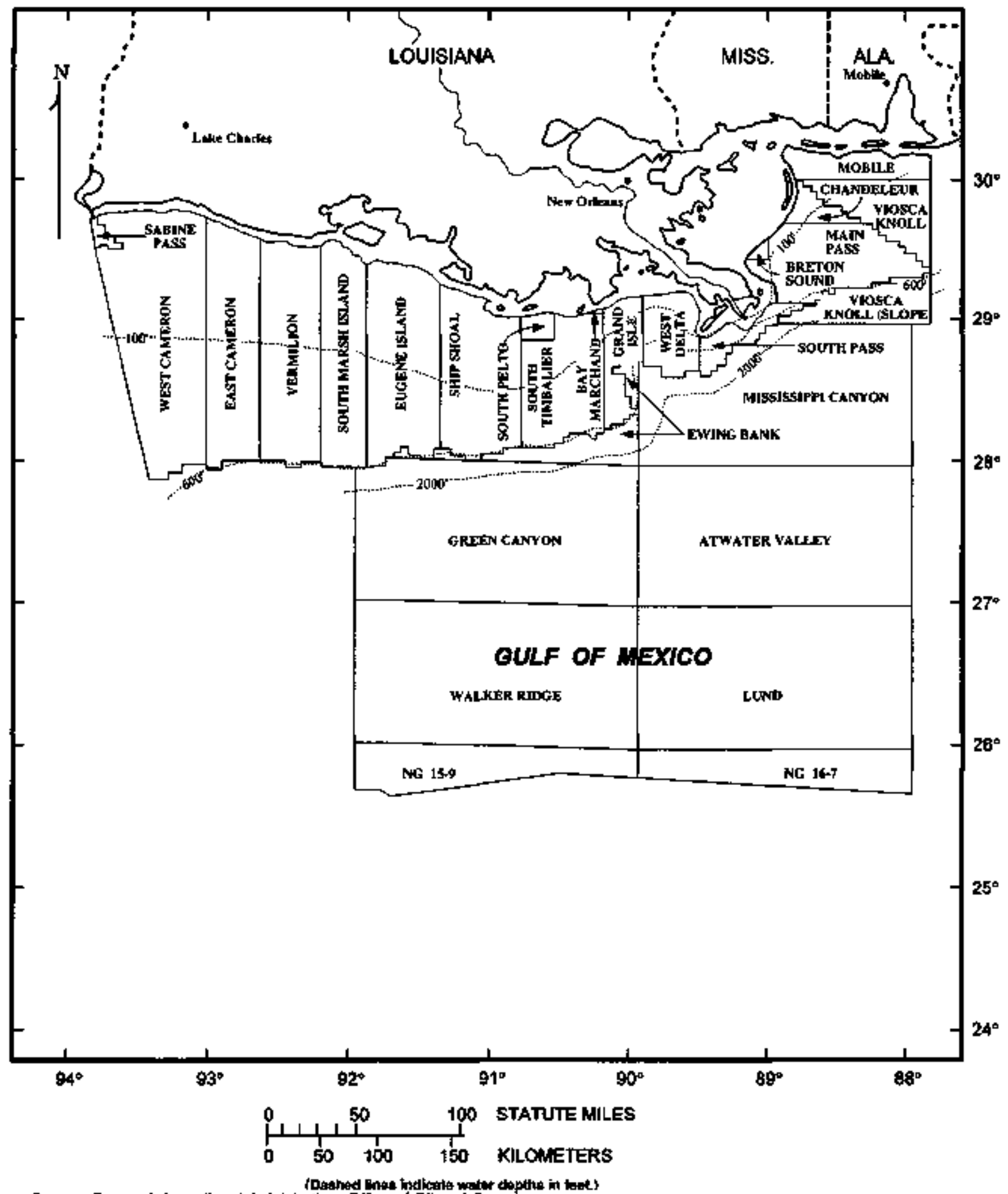

Source: Energy Information Administralion, Office of OA sond Cas. 
Figure H8. Eastern Planning Area, Gulf of Mexlco Outer Continental Shelf Region

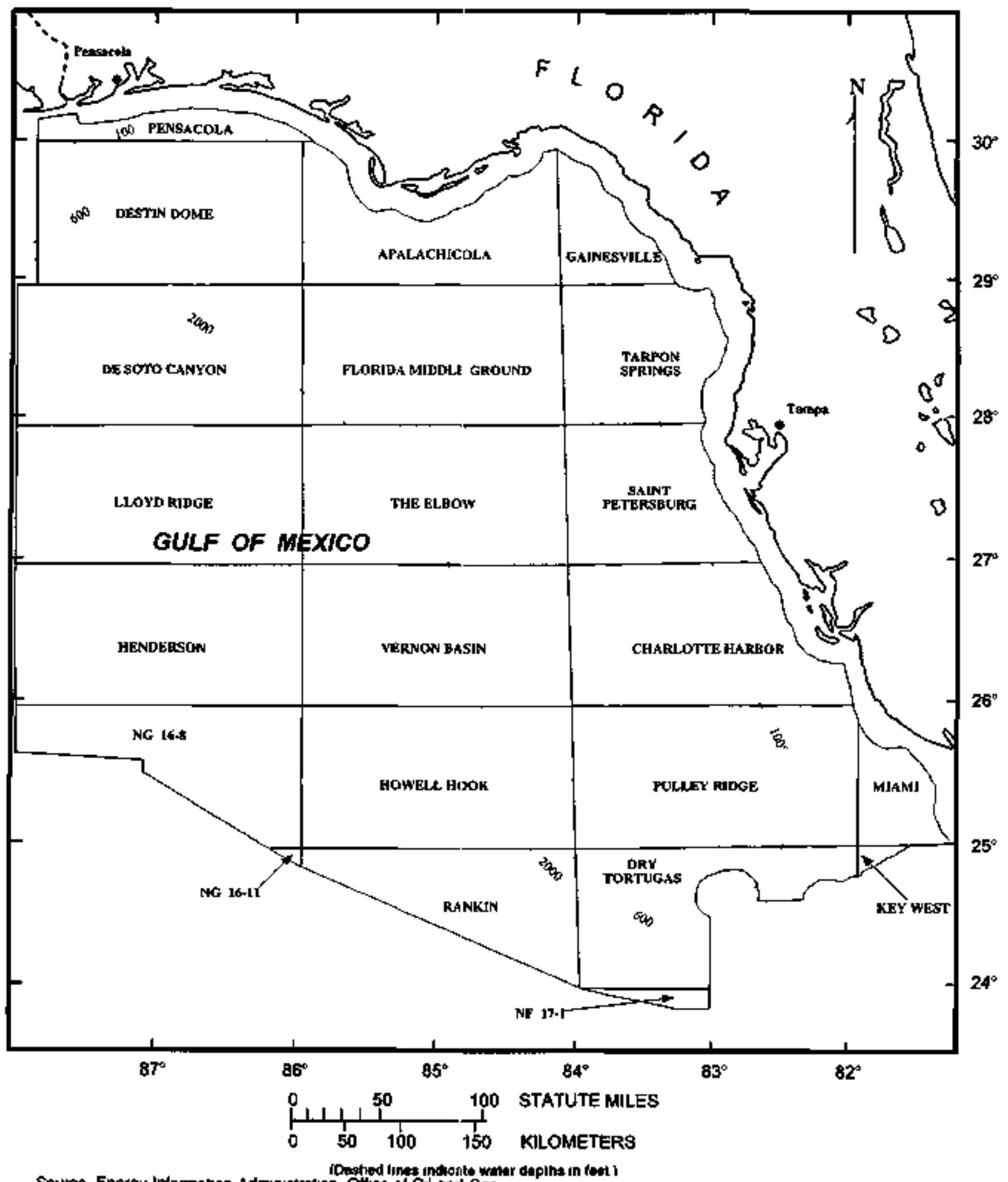

Source Energy Intormabon Admunistrailion Othce of oil ancl cas 
Appendix I

\section{Annual Survey Forms for Domestic Oil and Gas Reserves}



Figure 11. Form ElA-23, Cover Page

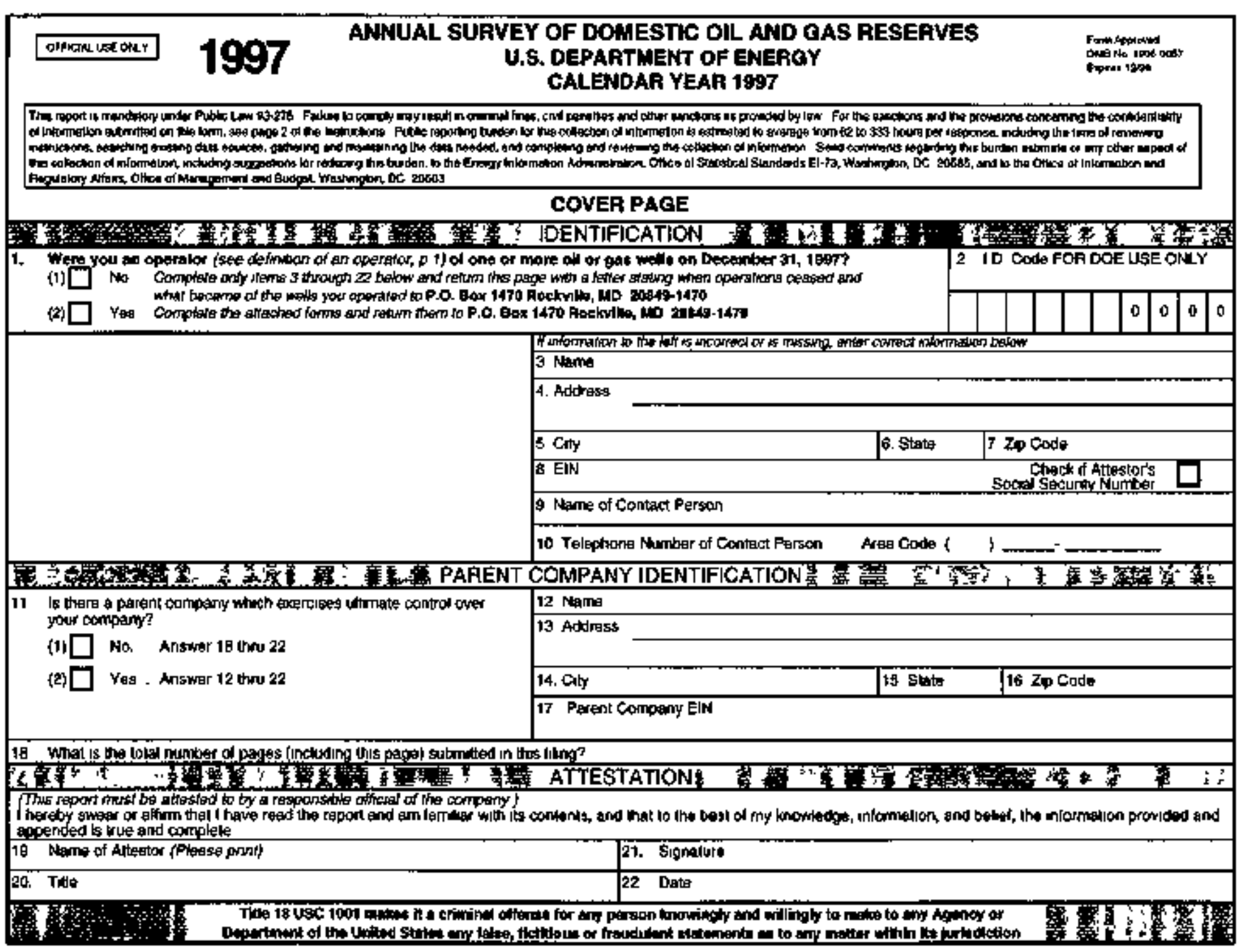

\section{FOR ASSISTANCE CALL 1-800-879-1470}


Figure 12. Form ElA-23, Summary Report - Page 1

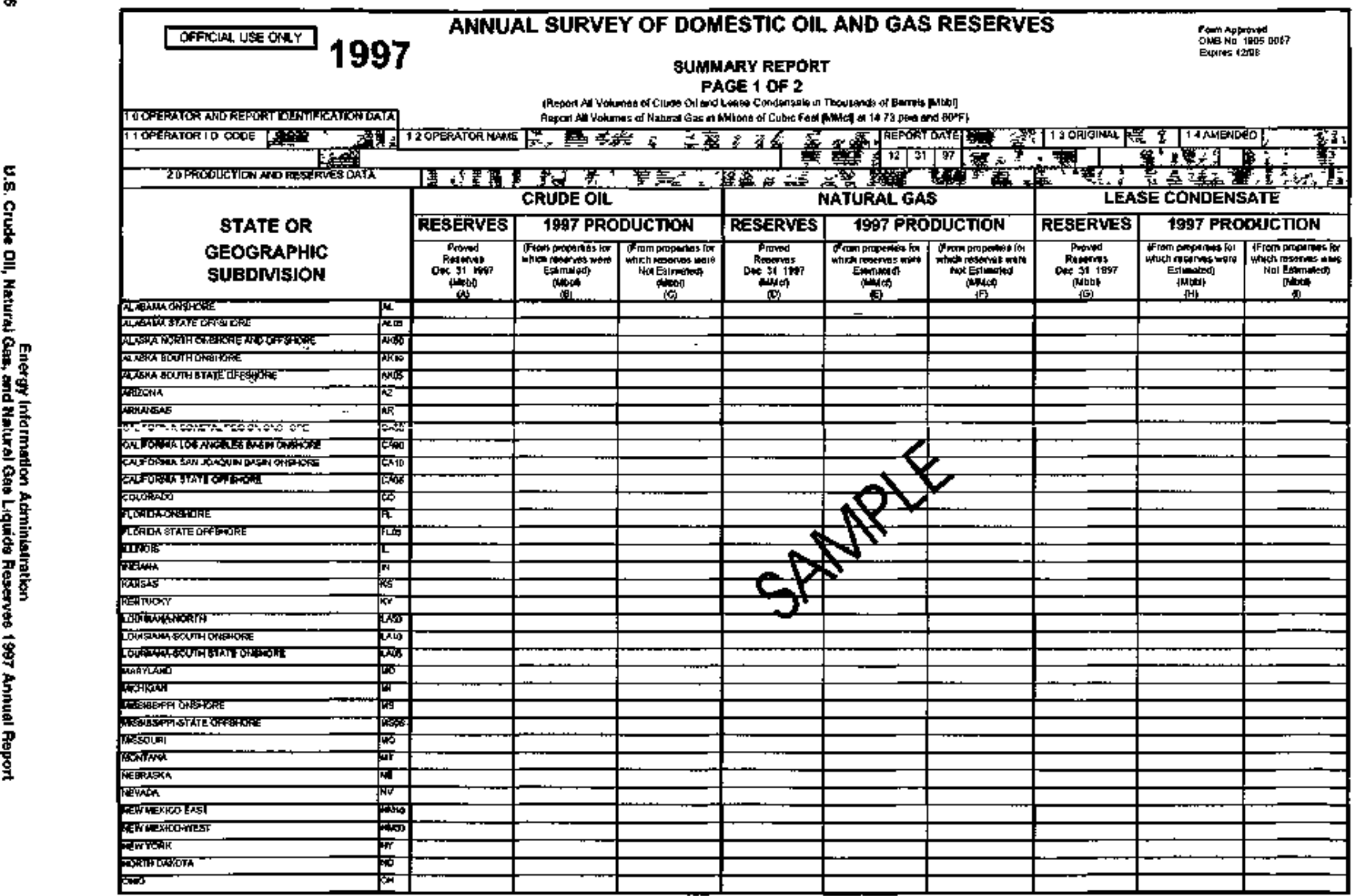

Source. Energy Inidomalion Administralum, Office of Oil and Gas 
Figure 13. Form ElA-23, Summary Report - Page 2

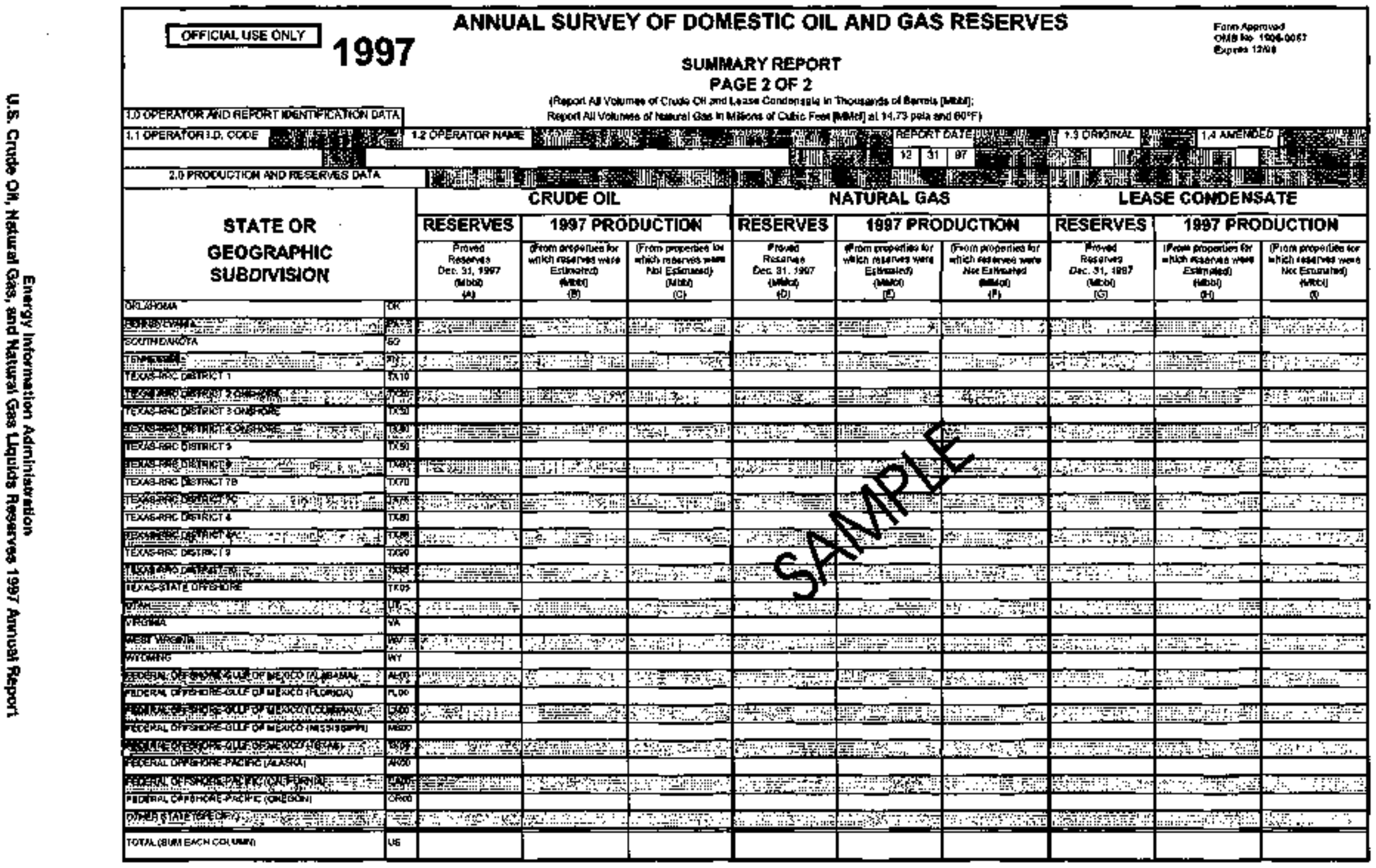


Figure 14. Form ElA-23, Detall Report - Schedule A

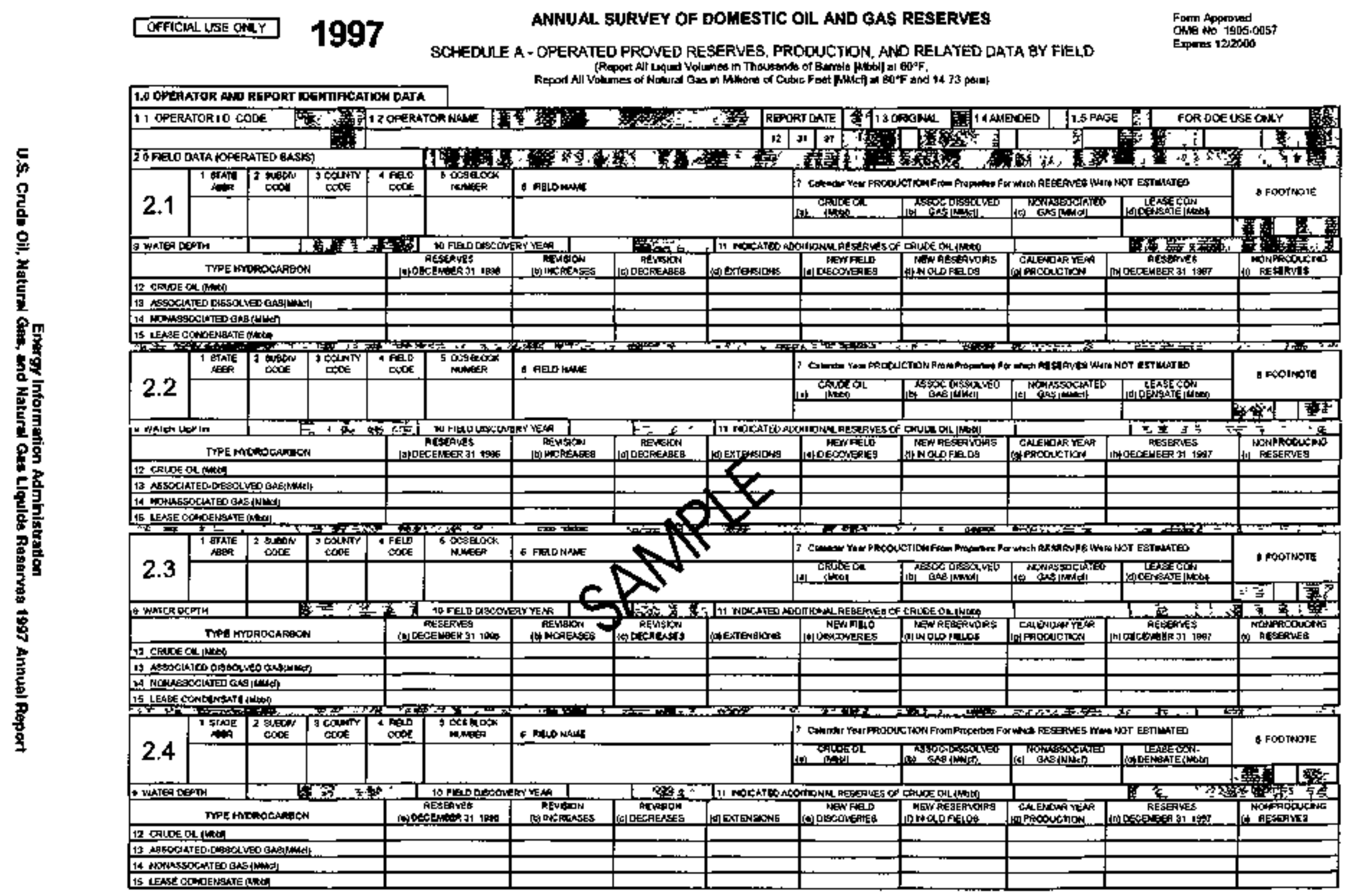

Soumes: Eneroy Mormation Administration, Ohice of Oll and Gas. 
Figure 15. Form EIA-23, Detall Report - Schedule B

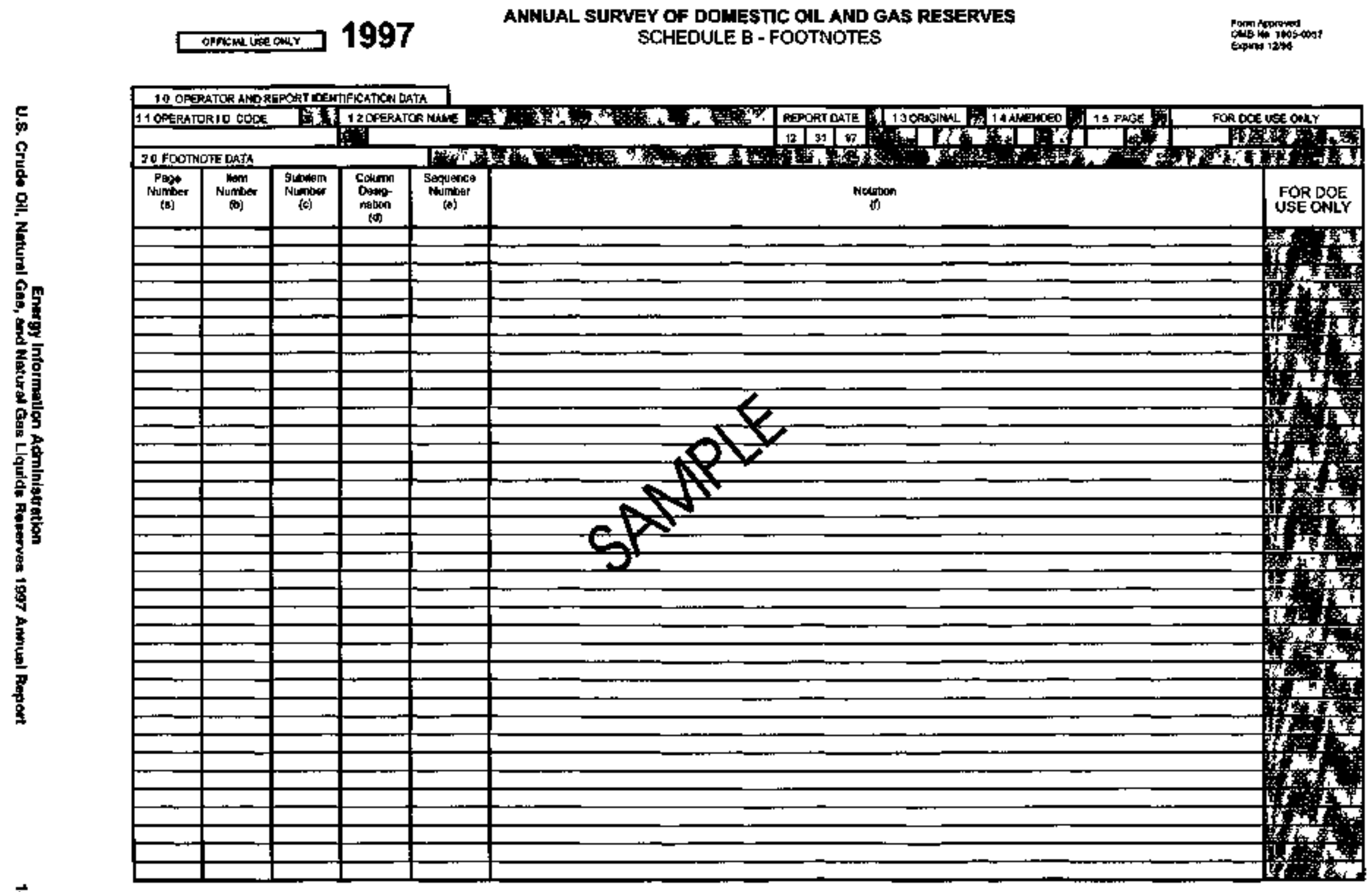


ELAAAA [ReVIged OMPI]

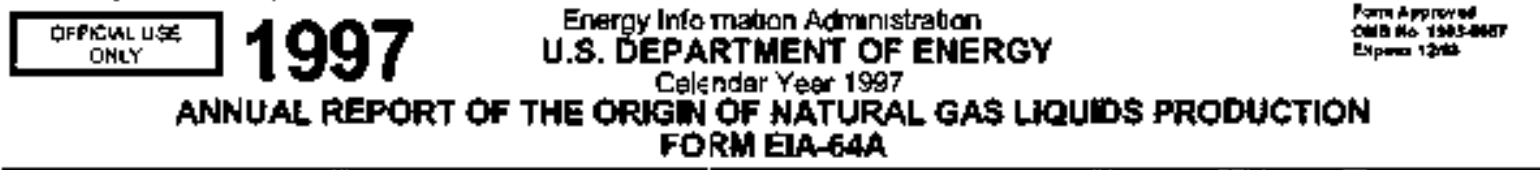

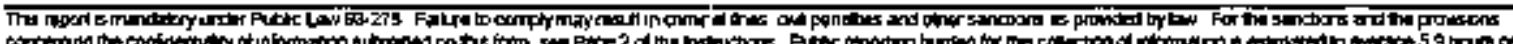

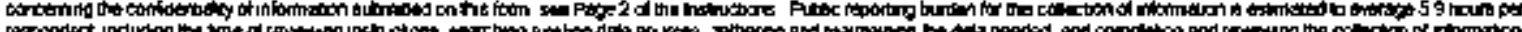

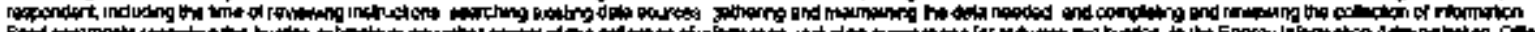
5.

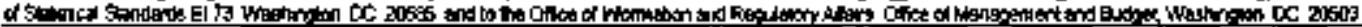

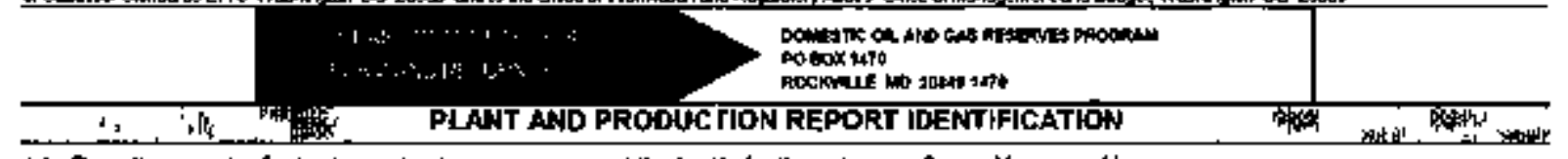

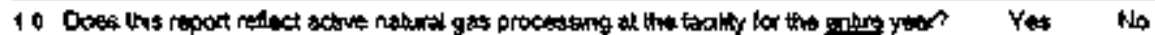

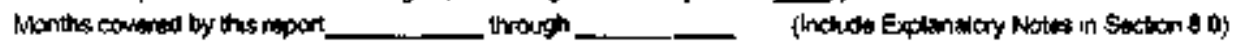

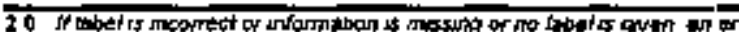

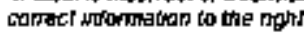

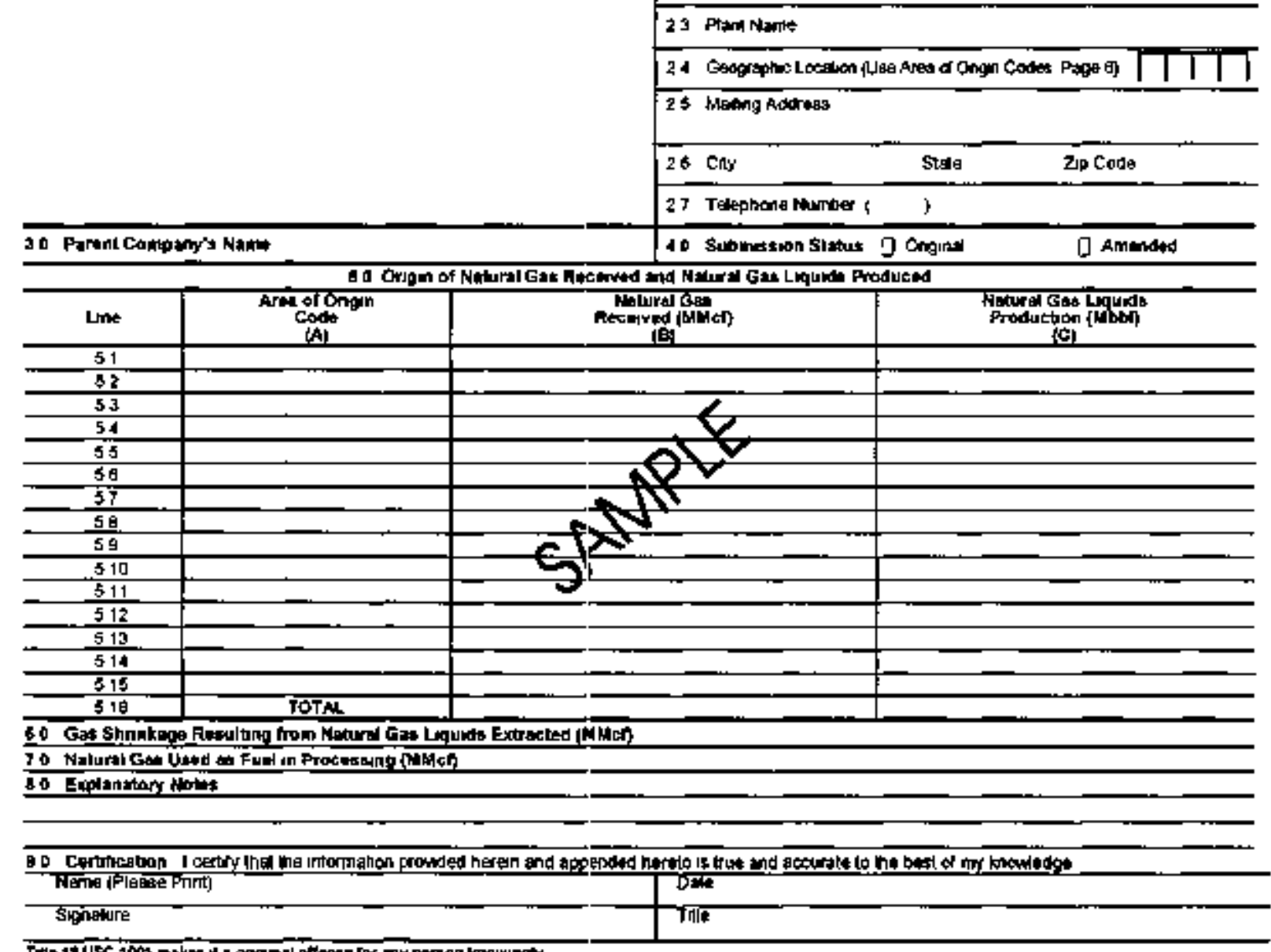

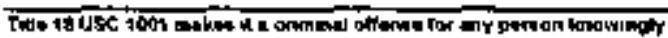

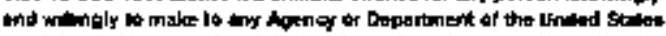

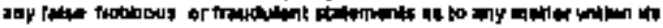
תundalim

\section{FOR ASSISTANCE CALL 1-900-879-1470}

Soume Energy Intormalion Admurasiration, Offfice of $Q_{11}$ and $G$ त्र 
Glossary 



\section{Glossary}

This glossary contains definitions of the technical terms used in this report and employed by respondents in completing Form ELA--23, "Annual Survey of Domestic Oil and Gas Reserves," or Form ElA-64A, "Annual Report of the Origin of Natural Gas Liquids Production, for the report year 1995.

Adjustments: The quantity which preserves an exact annual reserves balance within each State or State subdivision of the following form:

$$
\begin{aligned}
& \text { Puba|shod Prowed Peserves at End of Previous Peport Yom } \\
& + \text { Ad'ustments } \\
& + \text { Revisten Increases } \\
& \text { - Rovision Decreases } \\
& + \text { Extensions } \\
& \text { + Naw Flatd Dlscoverles } \\
& + \text { New Reservoir Discoveries in Old Fields } \\
& + \text { Report Yoar Production } \\
& \text { = Published Prowed Reservos at End of Report Year }
\end{aligned}
$$

These adjustments are the yearly changes in the published reserve estimates that cannot be attributed to the estimates for other reserve change categories because of the survey and statistical estimation methods employed. For example, variations as a result of changes in the operator frame, different random samples or imputations for missing or unreported reserve changes, could contribute to adjustments.

Affiliated (Associated) Company: An "affiliate" of, or a person "affiliated" with, a specific person is a person that directiy, or indirectly through one or more intermediaries: controls; or is controlled by; or is under common control with, the person specified. (See Person and Control)

Control: The term "control" (including the terms "controlling," "controlled by," and "under common control with") meats the possession, direct or indirect, of the power to direct or cause the direction of the management and policies of a person, whether through the ownership of voting shares, by contract, or otherwise. (See Person)

\section{Corrections: (See Revisions)}

Crude Oil: A mixture of hydrocabons that exists in the liquid phase in natural underground reservoirs and remains lìquid at atmospheric pressure aftet passing through surface separating facilities. Crude oil may also include:

1. Small amounts of hydrocarbons that exist in the gaseous phase in natural underground reservoirs but are liquid at atmospheric pressure after being recovered from oil weli (casinghead) gas in lease separators, and that subsequently are comingled with the gude stream without being separately measured

2. Small amounts of nonhydrocarbons produced with the oil.

When a State regulatory agency specifies a definition of crude oil which differs from that set forth above, the State definition is to be followed and its use footnoted on Schedule B of Form ElA-23.

Extensions: The reserves credited to a reservoir because of enlargenent of its proved area. Normally the ultimate size of newly discovered fields, or newly discovered reservoirs in old fields, is determined by wells drilled in years subsequent to discovery. When such wells add to the proved area of a previously discovered reservoir, the increase in proved reserves is classified as an extension.

Field: An area consisting of a single reservoir or multiple reservoirs all grouped on, or related to, the same individual geological structural feature and/or stratigraphic condition. There may be two or more reservoirs in a field that are separated vertically by intervening impervious strata, or laterally by local geologic barriers, or by both.

Field Area: A geographic area encompassing two or more pools that have a common gathering and metering system, the reserves of which are reported as a single unit. This concept applies primarily to the Appalachian region. (See Pool)

Field Discovery Year: The calendar year in which a field was first recognized as containing economically recoverable accumulations of oil and/or gas.

Field Separation Facility: A sutface installation designed to recover lease condensate from a 
produced natural gas stream frequently originating from more than one lease, and managed by the operator of one or more of these leases. (Set Lease Condensate)

Gross Working Interest Ownership Basis: Gross working interest ownership is the respondent's working interest in a given property plus the proportionate share of any royalty interest, including overriding royalty interest, associated with the working interest. (See Working Interest and Royalty (including Overriding Royalty) Interest)

Indicated Additional Reserves of Crude Oil: Quantities of crude oil (other than proved reserves) which may become economically recoverable trom existing productive reservoirs through the application of improved recovery techniques using current technology. These recovery techniques may.

1. Already be installed in the reservoir, but their effects are not yet known to the degree necessary to classify the additional reserves as. proved

2. Be installed in another similar reservoir, where the results of that installation can be used te estimate the indicated additional reserves.

Indicated additional reserves are not included in proved reserves due to their uncertain economic recoverability. When economic recoverability is demonstrated, the indicated additional reserves must be transferred to proved reserves as positive revisions.

Lease Condensate: A mixture consisting primatrily of pentanes and heavier hydrocarbons which is recovered as a liquid trom natural gas in lease or field separation facilities, exclusive of products recovered at natural gas processing plants or facilities.

Lease Separator: A lease separator is a facilty installed at the surface for the purpose of (a) separating gases from produced crude oil and water at the temperature and pressure conditions of the separator, and/or (b) separating gases from that portion of the produced natural gas stream which liquefies at the temperature and pressure conditions of the separator.

Natural Gas: A mixture of hydrocarbon compounds and small quantities of various nonhydrocarbons existing in the gaseous phase or in solution w.th crude oil in natural underground reservoirs at reservoir conditions. The principal hydrocarbons nomally contained in the mixture are methane, ethane, propane, butane, and pentanes. Typical nonhydrocarbon gases which may be present in reservoir natural' gas are wates vapor, carbon dioxide, helium, hydrogen suifide, and nitrogen. Under reservoit condilions, natural gas and the liquefiable portions occur either in a single gaseous phase in the reservoir or in solution with crude oil, and are not distinguishable at the time as separate substances, (See Natural Gas, Associated-oDissolved and Natural Gas, Nonassociated)

Natural Gas, Associated-Dissolved: The combined volume of natural gas which occurs in crude oil reservoirs either as free gas (associated) or as gas in solution with crude oil (dissolved).

Natural Gas, "Dry": The actual or calculated volumes of natural gas which remain after.

1. The liquefiable hydrocarbon portion has been removed from the gas stream (i.e., gas after lease, field, and/or plant separation)

2. Any volumes of nonhydrocarbon gases have been removed where they occur in sufficient quantity to render the gas unmarketable.

Natural Gas, Nonassociated: Natural gas not in contact with significant quantities of crude oil in a reservoir.

Natural Gas Liquids: Those hydrocarbons in natural gas which are separated from the gas through the processes of absorption, condensation, adsorption, or other methods in gas processing or cycling plants. Generally such liquids consist of propane and heavier hydrocarbons and are commonly referred to as condensate, natural gasoline, or liquefied petroleum gases. Where hydrocarbon components lighter than propane are recovered as liquids, these components are included with natural gas liquids.

Natura] Gas Processing Plant: A facility designed to recover natural gas liquids from a stream of natura? gas which may or may not have passed through lease separators and/or field separation facilities. Another function of the facility is to control the quality of the processed natural gas stream. Cycling plants are considered natural gas processing plants.

Natural Gas, Wet After Lease Separation: The volume of natural gas remaining after removal of lease condensate in lease and/or field separation facilities, if any, and after exclusion of 
nonhydrocarbon gases where they occur in sufficient quantity to render the gas tunmarketable. Natural gas liquids may be secovered from volumes of natural gas, wet after lease separation, at natural gas processing plants. (See Lease Condensate, Lease Separator, and Field Separation Facility)

Net Revisions: (See Revisions)

New Field: A field discovered during the report year.

New Field Discoveries: The volumes of proved reserves of crude oil, nahural gas and/or natural gas liquids discovered in new fields during the report year.

New Reservoir A reservoir discovered during the report year.

New Reservoir Discoveries in Old Fields: The volurnes of proved reserves of crude oil, natural gas, and/or natural gas liquids discovered during the report year in new reservoir(s) located in old fields.

Nonproducing Reservoirst Reservoirs in which proved liquid or gaseous hydrocarbon seserves have been identified, but which did not produce during the last calendar year regardless of the availability and/or operation of production, gathering, or transportation facilities.

Old Field: A field discovered prior to the report year.

Old Reservoir: A reservoir discovered prior to the report year.

Operator, Gas Plant: The person responsible for the management and day--to-day operation of one or more natural gas processing plants as of December 31 of the report year. The operator is generally a working interest owner or a company under contract to the working interest owner(s). Plants shut down during the report year are also to be considered "operated"t as of December 31. (See Person)

Operator, Oil and/or Gas Well: The person responsible for the management and day-to-day operation of one or more crude oil and/or natural gas wells as of December 31 of the report year. The operator is generally a working interest owner or a company under contract to the working interest owner(s). Wells included are those which have proved reserves of crude oil, natural gas, and/or lease condensate in the reservoirs associated with them, whether or not they are producing. Wells abandoned during the report year ate also to be considered "operated" as of December 31. (See Person, Proved Reserves of Crude Oil, Proved Reserves of Natural Gas, Proved Reserves of Lease Condensate, Report Year, and Reservoir)

\section{Ownership: (See Gross Working Interest Ownership Basis)}

Parent Company: The parent company of a business entity is an affiliated company which exercises ultimate control over that entity, either directly or indirectly through one or more intermediaries. (See Affiliated (Associated) Company and Control)

Person: An individual, a corporation, a partnership, an association, a joint-stock company, a business trust, or an unincorporated organization.

Poal: In general, a reservoir. In certain situations a pool may consist of more than one reservoir. (See Field Area)

Plant Liquids: Those volumes of natural gas liquids recovered in natural gas processing plants.

Production, Crude Oil: The volumes of crude oil which are extracted from oil reservoirs during the report year. These volumes are determined through measurement of the volumes delivered from lease storage tanks, (i.e, at the point of custody transfer) with adjustment for (1) net differences between opening and closing lease inventories, and for (2) basic sedinent and water. Oil used on the lease is considered production.

Production, Lease Condensate: The volume of tease condensate produced during the report year. Lease condensate volumes include only those volumes recovered from lease or field separztion facilities. (See Lease Condensate)

Production, Natural Gas, Dry: The volume of natural gas withdrawn from reservoirs during the report year less (I) the volume rekurned to such reservoirs in cycling, repressuring of oil reservoirs and conservation operations; less (2) shrinkage resulting from the removal of lease condensate and plant liquids: and less (3) norhydrocarbon gases where they occur in sufficient quantity to render the gas unmarketable. Volumes of gas withdrawn from gas storage reservois and natlve gas, which has been transferred to the storage category, are not 
considered production. This is not the same as marketed production, since the latter also excludes vented and flared gas, but contains plant liquids.

Production, Natural Gas, Wet after Lease Separation: The volume of natural gas withdrawn from reservoirs during the report year less (1) the volume returned to such reservoirs in cycling. repressuring of oil reservoirs and conservation operations; less (2) shrinkage resulting from the removal of lease condensate; and less i3) nonhydrocarbon gases where they occur in sufficient quantity to render the gas unmarketable. Volumes of gas withdrawn from gas storage reservoirs and native gas, which has been transferred to the storage category, ate not considered production. This is not the same as marketed production, since the katter excludes vented and flared gas.

Production, Natural Gas Liquids: The volume of natural gas liquids removed from natural gas in lease separators, field facilities, gas processing plants or cycling plants during the report year.

Production, Plant Liquids: The volume of liquids removed from natural gas in natural gas processing plants or cycling plants during the report year.

Proved Reserves of Crude Oil; Proved reserves of crude oil as of December $\mathbf{3 1}$ of the report year are the estinated quantibies of all liquids defined as crude oil, which geological and engmeering datta demonstrate with reasonable certainty to be recoverable in future years from known reservoirs under existing economic and operating conditions.

Reservoirs are considered proved if economic producibility is supported by actual production or conclusive formation test (drill stem or wire line), or if economic producibility is supported by core analyses and/or electric or other log interpretations. The area of an oil reservoir considered proved includes (1) that portion delineated by drilling and defined by gas-oil and/or oif-water contacts, if any; and (2) the immediately adjoining portions not yet drilled, but which can be reasonably judged as economically productive on the basis of available geological and engineering data. In the absence of information on fluid contacts, the lowest known structural occurrence of hydrocarbons is considesed to be the lower proved limit of the reservoir.

Volumes of crude oil placed in underground storkige are not to be considered proved reserves.
Reșerves of cnude oil which can be produced economically through application of improved recovery techniques (such as fluid injection) are included in the "proved" classification when successful testing by a pilot project, or the operation of an installed program in the reservoir, provides support for the engineering analysis on which the project or program was basted.

Estimates of proved crude oil reserves do not include the following: (1) oil that may becone available from known reservoirs but is reported separately as "indicated additional reserves"; (2) natural gas liquids (including lease condensate); (3) oil, the recovery of which is subject to reasonable doubt because of uncertainty as to geology, reservoir characteristics, or economic factors; (4) oil that may occur in undrilled prospects; and (5) oil that may be recovered from oil shales, coal, gilsonite, and other such sources. It is not necessary that production, gathering or transportation facilities be installed or operative for a reservoir to be considered proved.

Proved Reserves of Lease Condensate: Proved reserves of lease condensate as of Decenber 31 of the report yeas ase the volumes of lease condensate expected to be recovered in future years in conjunction with the production of proved reserves of natural gas as of December 31 of the report year. based on the recovery efficiency of lease and/or field separation facilities installed as of December 31 of the report year. (See Lease Condensate and Proved Reserves of Natural Gas)

Proved Reserves of Natural Gas: Proved reserves of natural gas as of December $\mathbf{3 1}$ of the report year are the estimated quantities which analysis of geological and engineering data demonstrate with reasonable certainty to be recoverable in future years from known reservoirs under existing economic and operating conditions.

Reservoirs are considered proved if economic producibility is supported by actual production or conclusive formation test (drill stern or wire line), or if economic producibility is supported by core analyses and/or electric or other log interpretations.

The area of a gas reservoir considered proved includes: (1) that portion delineated by drilling and defined by gas-oil and/or gas-water contacts, if any; and (2) the immediately adjoining portions not yet drilled, but which can be reasonably judged as economically productive on the basis of available geological and engineering data. In the absence of 
information on fluid contacts, the lowest known structural occurrence of hydrocarbons is considered to be the lower proved linit of the reservoir.

Volumes of natural gas placed in underground storage are not to be considered proved reserves.

For natural gas, wet after lease separation, an appropriate reduction in the reservoir gas volume has been made to cover the removal of the liquefiable portions of the gas in lease and/or field separation facilities and the exclusion of nonhydrocarbon gases where they occur in sufficient quantity to render the gaș unmarketable.

For dry natural gas, an appropriate reduction in the gas volume has been made to cover the removal of the liquefiable portions of the gas in lease and/or field separation facilities, and in natural gas processing plants, and the exclusion of nonhydrocarton gases where they occur in sufficient quantity to render the gas unmarketable.

It is not necessary that production, gathering or transportation facilities be instatled or operative for a reservoir to be considered proved. It is to be assumed that compression will be initiated if and when economically justified.

Proved Reserves of Natural Gas Liquids: Proved reserves of natural gas liquids as of December 31 of the report year are those volumes of natural gas liquids (including lease condensate) demonstrated with reasonable certrinty to be separable in the future from proved natural gas reserves, under existing economic and operating conditions.

Proved Ultimate Recovery: The sum of proved reserves and cumulative production. It is expected to change over time for any field, group of fields, State, or Country. Froved Ultimate Recovery does not represent the maximum recovetable volume of resources for an area. It is instead a gauge of how much has already been produced plus proved reserves. Proved reserves of crude oil or natural gas are the estimated quantities of petroleum which geological and engineering data demonstrate with reasonable certainty to be recoverable in future years from known reservoirs under existing economic and operating conditions. When deterministic proved reserves estimation methods are used, the term reasonable certainty is intended to express a high degree of confidence that the estimated quantities will be recovered. When probabilistic methods are used there should be at least a 90 percent probability that the actual quantities recovered will exceed the estimate.

Report Year. The calendar year to which data reported in this publication pertain.

\section{Reserves: (See Proved Reserves)}

Reserve Additions: Consist of adjustments, net revisions, extensions to old reservoirs, new reservoir discoveries in old fields, and new field discoveries.

Reserves Changes: Positive and megative revisions, extensions, new reservoir discoveries in old fields, and new field discoveries, which occurred during the report year.

Reservoir: A porous and permeable umderground formation conkaining an individual and separate natural accurrulation of producible hydrocarbons (oil and/or gas) which is confined by inpermeable rock or water barriers and is characterized by a single natural pressure system.

Revisions: Changes to prior year-end proved reserves estmates, either positive or negative, resulting from new information other than an increase in proved acreage (extension). Revisions include increases of proved reserves associated with the installation of improved recovery techniques or equipment. They also include correction of prior report yeas arithmetical or clerical errors and adjustments to prior year-end production volumes to the extent that these aiter reported prior year reserves estimates.

Royalty (Including Overriding Royalty) Interests: These interests entitle their owner(s) to a share of the mineral production from a property or to a share of the proceeds therefrom. They do not contain the rights and obligations of operating the property, and normally do not bear any of the costs of exploration, development, and operation of the property.

Subdivision: A prescribed portion of a given State or other geographical region defined in this publication for statistical reporting purposes.

Sabsidiary Company: A company which is controlled through the ownership of voting stock, or a corporate joint venture in which a corporation is owned by a small group of businesses as a separate and specific business or project for the mutual benefit of the merrbers of the group. (See Control) 
Total Discoveries: The sum of extensions, new reservoir discoveries in old fields, and new field discoveries, which occurred during the report year.

Total Liquid Hydrocarbon Reserves: The sum of crude oil and natural gas liquids reserves volumes.

Total Operated Basis: The total reserves or production associated with the wells operated by an individual operator. This is also commonly known as the "gross operated" or " $8 / 8$ ths" basis.

Working Interest: A working interest permits the owner(s) to explore, develop and operate a property. The working interest owner(s) bear(s) the costs of exploration, development and operation of the property, and in retum is (are) entitled to a share of the mineral production from the property or to a share of the proceeds therefrom. 
Nearly all the ifems described below, and many others, can be accessed wit the Energy Information Administration's World wide Web site at hitp'lhww ela.doe gov; chck on "Environmenl." Some items are also available in hard copy. For further infombtion on these and hundreds of olher ElA products, contact the Natlonal Energy Infomalion Center at infoctr@eia.doe.gov or 202-588- 8800 .

\section{Emissions of Greenhouse Gases in the United States 1997}

Annual inventory of human-caused greenhouse gases in the United States.

\section{Annual Energy Outook 1998}

Projections through 2020 of energy supply, demand, and price, including projected emissions of carbon, sulfur dioxide, and nitrogen oxide from energy use and electricity generation.

Mitigating Greenhouse Gas Emissions: Volutuaty Reporting

Summary of data reported to the Voluntary Reporting of Greenhouse Gases Program.

Energy U/se and Carbon Emissions: Some International Comparisons

Comparisons of energy use pattems and carbon emissions in member countries of the Organization for Economic Cooperation and Development.

\section{Energy Use and Carbon Emissions: Non-OECD Countries}

Encrgy use patterns and carbon emissions in countries that are not members of the Organization for Economic Cooperation and Development.

\section{State-Level Carbon Dioxide Emixsion Factors for Coal, 1980-1995}

Emission factors from the State Energy Data Report 1995, which also includes state consumption data useful for estimating state carbon dioxide emissions.

\section{An Analysis of FERC's Final Environmental Impact Statement for Electricity: Open Access and Recovery of Siranded Costs \\ Trends in emissions, utility fuel use, and electricity trading.}

The Effects of Title IV of the Clean Air Act Amendments of 1990 on Electric Uthities:

An Update

Strategies used by electric utilities to comply with the Acid Rain Program in 1995, costs of

compliance, and effects on $\mathrm{SO}_{2}$ emissions and coal supply and dernand.

\section{Etectric Utilty Phase I Acid Rain Compliance Strategies for the}

\section{Clean Air Act Amendments of 1990}

Strategies used and costs incurred by selected electric utilities in complying with Phase I (1995-1999) of the Clean Air Act Amendments of 1990. (Not available electronically; contact NEIC for ordering information.) 
En. "gy Information Administration

U.S. Department of Energy

Forrestal Building, El-30

Washington, DC 20585

OFFICIAL BUSINESS

Do Not Forward, Do Nol Return

Address Correction Requested

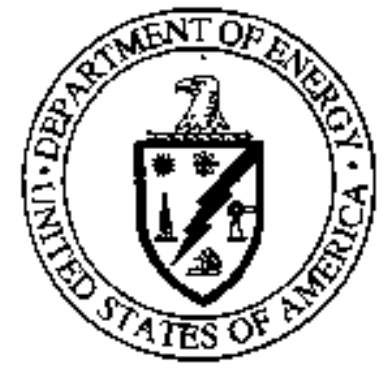

